\title{
Lossen Reaction, Polymer Synthesis and Potential Applications of Latent Isocyanates
}

By

Khama Rani Ghosh

\begin{abstract}
A thesis submitted to
the Faculty of Graduate Studies and Postdoctoral Affairs

in Partial Fulfillment of the Requirements for the Degree of

Doctor of Philosophy

in

Chemistry
\end{abstract}

Carleton University

Ottawa, ON, Canada

April 2017

(C) Copyright, Khama Rani Ghosh, Ottawa, ON, Canada, 2017 


\begin{abstract}
Isocyanates are of high interest for their versatile reactions and industrial uses in agrochemicals (e.g., herbicides) and polymers (e.g., polyurethanes and polyureas). The main objectives of this thesis research are to study the Lossen reaction of the $N$-acetoxy amide (AA) as a latent isocyanato group and explore potential applications of AA-containing compounds and polymers. Another objective is to develop a new method for optical sensing of isocyanates in air and as well nitroaromatic compounds.
\end{abstract}

Study and applications of the Lossen reaction of a compound equivalent to an industrially important diisocyanate are described. The conversion of the AA group in a precursor monomer to the isocyanato group under various conditions was studied. In the presence of a polyol, polyurethanes or AA-terminated prepolymers could be formed, depending on the reaction conditions (e.g., temperatures and a base catalyst). In addition, the synthesis of AA-containing polymethacrylates and thermal conversion of the AA group to the reactive isocyanato group are illustrated. The AA-containing methacrylate monomer was prepared and polymerized with methyl methacrylate to form a series of copolymers with a range of the AA content. These copolymers were characterized by IR, ${ }^{1} \mathrm{H}$ NMR and ${ }^{13} \mathrm{C}$ NMR spectroscopy, thermogravimetric analysis, differential scanning calorimetry and gel permeation chromatography. Treatment of these copolymers at $110-130{ }^{\circ} \mathrm{C}$ led to the in situ formation of the isocyanato group and thus chemically reactive polymers.

A fluorescence quenching method is introduced for direct and sensitive detection of various aliphatic and aromatic isocyanates in air. The high sensitivity is attributed to high fluorescence of polymer $\mathbf{P 1}$ film as a result of its unique aggregation-induced emission. The isocyanates can be detected at the ppt level typically within 10-60 s under ambient conditions. Moreover, the further application of fluorescent polymer P1 for sensing nitroaromatic 
compounds in air and in solution is explored. A large fluorescence quenching $(98 \%)$ was found for picric acid with a concentration of $4.7 \times 10^{-6} \mathrm{M}$. In addition, 2,4-dinitrotoluene (DNT) at the ppb level in air could be detected within $60 \mathrm{~s}$. 


\section{Acknowledgements}

I would like to offer gratitude to my supervisor Professor Zhi Yuan (Wayne) Wang for accepting me as a graduate student in this group, selecting the topics and his guidance, encouragement, and suggestions given to me throughout my research work. I also appreciate him for his support and motivation during a hard time of my study and research.

I am happy to get this opportunity to express my gratitude to Dr. Jane Gao for her assistance on various problems during the research period.

I want to thank the members in Dr. Wang's group: Wen Hui Hao, Duo Li, Saif Mia, Yasaman Aghili, Di Zhang, Mirvat Sanaallah, Songqiu Zhou and Sukanta Kumar Saha for pleasant experience during the research period. Special thanks to Monica Anne Gill for proofreading of my dissertation. I would like to say thanks to Jim Logan for fixing different instruments in our lab and fixing my laptop as well as Tony O'Neil, Keith Bourque and Peter Mosher for supporting of instrumental technique.

I wish to thank my family and mother who raised me. I want to gratitude and many thanks to my husband Sukanta Kumar Saha for endless support, assistance, and motivation during my study and research work. My sweet son, Sreekanta Saha whose smiley face always inspires me and his ability to understand me at such a young age always provides me a great pleasure during my study. At the end, I want to say thanks to all the members in the department of chemistry, Carleton University for a nice and friendly environment. 


\section{Table of Contents}

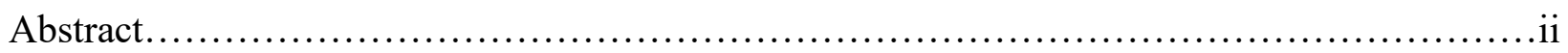

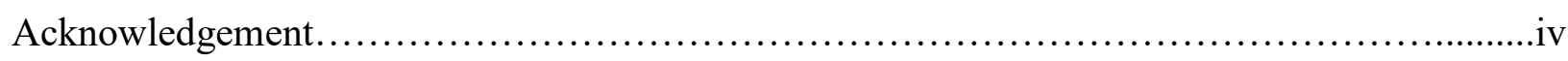

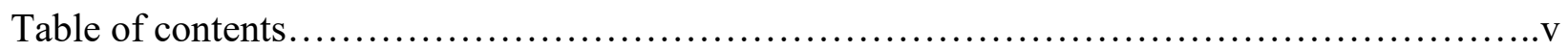

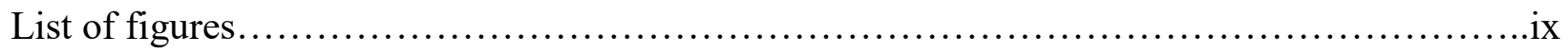

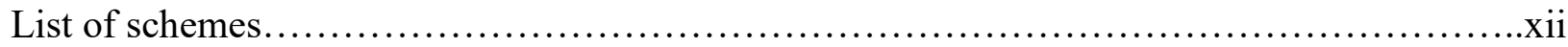

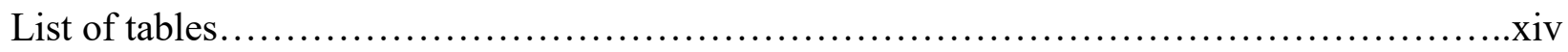

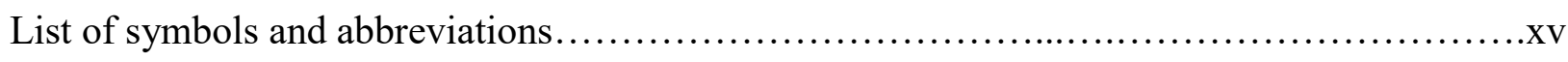

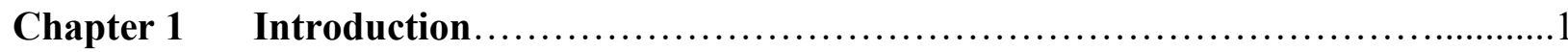

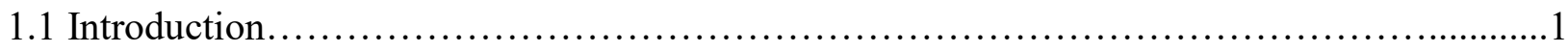

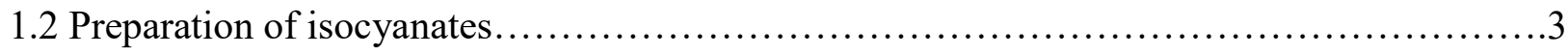

1.2.1 Preparation of isocyanates by phosgenation of amines..............................

1.2.2 Preparation of isocyanates from nitro compounds..................................

1.2.3 Preparation of isocyanates by Curtius rearrangement..............................

1.2.4 Preparation of isocyanates by Hofmann rearrangement.......................... 10

1.2.5 Preparation of isocyanates by Lossen rearrangement............................13

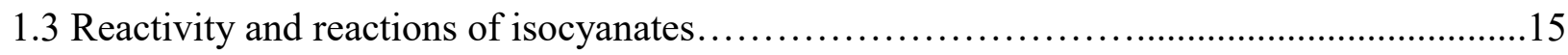

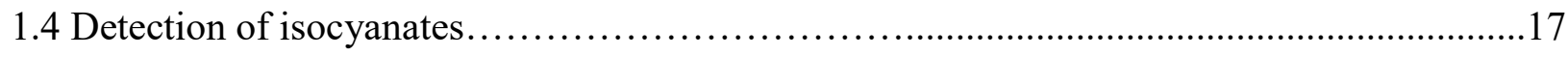

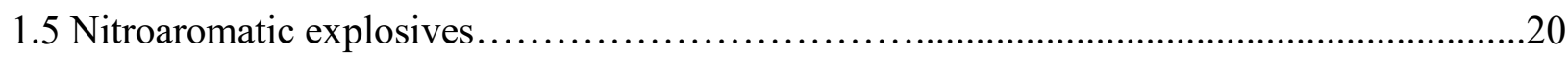

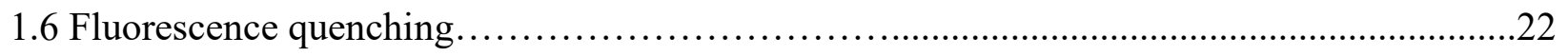

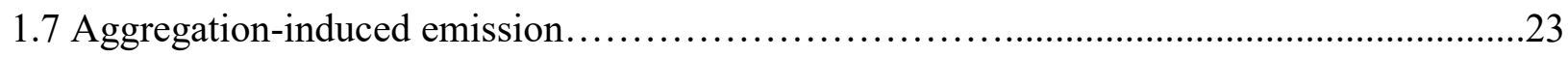

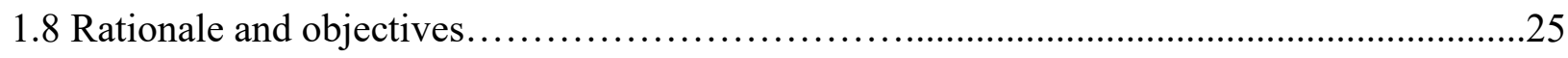

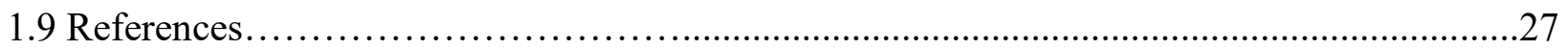




\section{Chapter 2 Synthesis and Characterizations of NCO-Terminated and $N$-Acetoxyamide-}

Terminated Prepolymers.................................................................40

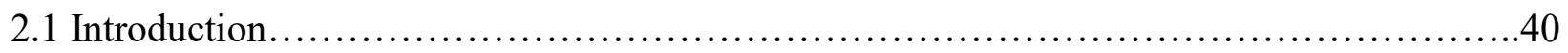

2.2 Synthesis and characterizations of $N^{1}, N^{4}$-dihydroxyterephthalamide.....................42

2.3 Synthesis and Characterizations of $N^{1}, N^{4}$-diacetoxyterephthalamide......................43

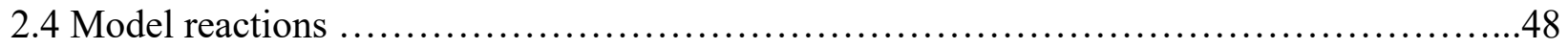

2.4.1 Reaction of $N^{1}, N^{4}$-diacetoxyterephthalamide and heptadecane-9-ol in absence of a base

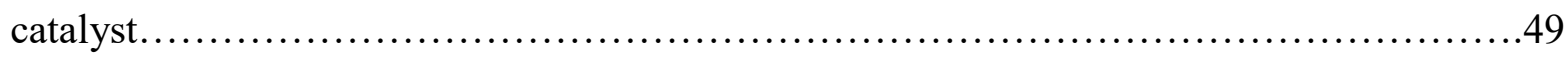

2.4.2 Reaction of $N^{1}, N^{4}$-diacetoxyterephthalamide and heptadecane-9-ol in the presence of a

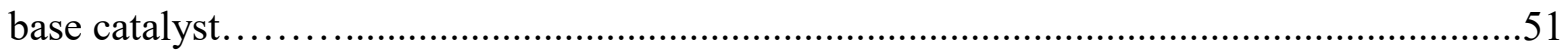

2.4.3 Reactions of $N$-acetoxybenzamide and $n$-butanol with and without a base catalyst.....53

2.5 Synthesis of NCO-terminated prepolymer from DATA and PTMEG $\ldots \ldots \ldots \ldots \ldots \ldots \ldots . . . .55$

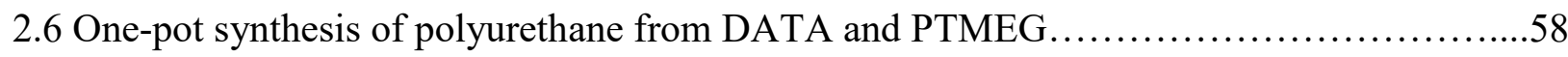

2.7 Synthesis of acetoxyamide-terminated prepolymer from DATA and PTMEG..............60

2.8. One-pot synthesis of polyurethane from DATA..................................62

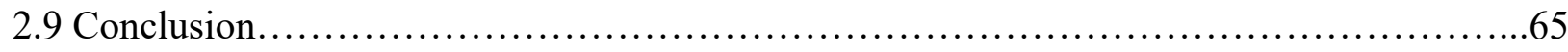

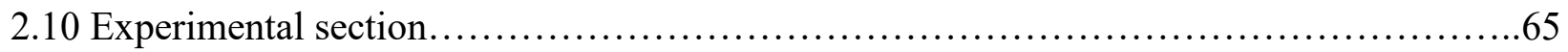

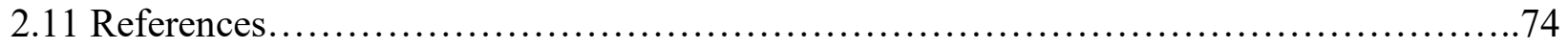

Chapter 3 Design, Synthesis, and Characterizations of Pendent NCO-containing

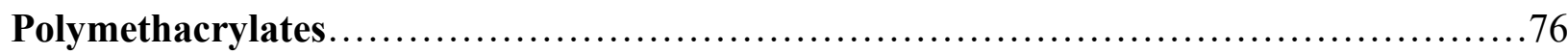

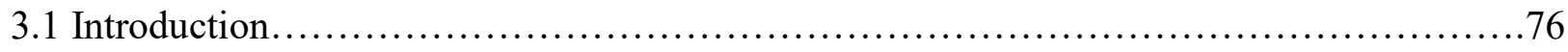

3.2 Synthesis and characterizations of $N$-hydroxymethacrylamide $\ldots \ldots \ldots \ldots \ldots \ldots \ldots \ldots \ldots . . .78$

3.3 Synthesis and Characterizations of $N$-acetoxymethacrylamide........................ 79

3.4 Synthesis and characterizations of MMA-AMAA copolymers $\ldots \ldots \ldots \ldots \ldots \ldots \ldots \ldots \ldots \ldots \ldots . . .22$ 
3.5 Conversion of $N$-acetoxyamide to $\mathrm{NCO}$ in MMA-AMAA copolymers..................86

3.6 Synthesis and characterizations of MMA-AMAA-HEMA terpolymers....................91

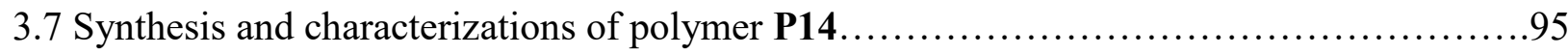

3.8. Study of self-crosslinking NCO- and $\mathrm{OH}$-containing polymer.......................96

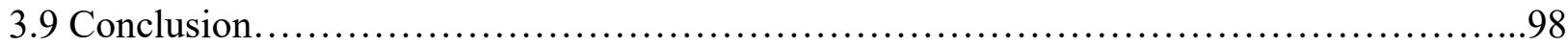

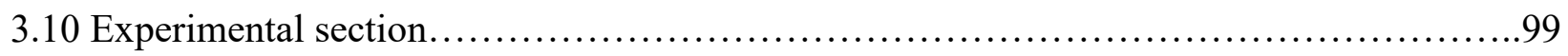

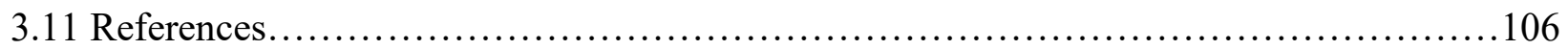

Chapter 4 Detection of Ultralow Trace Amounts of Isocyanates in Air by Fluorescent Conjugated Polymer....................................................... 108

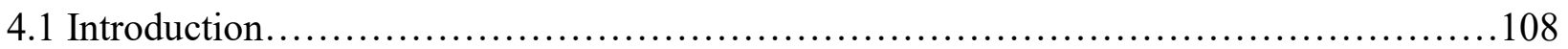

4.1.1 Overview of conjugated polymers..................................... 108

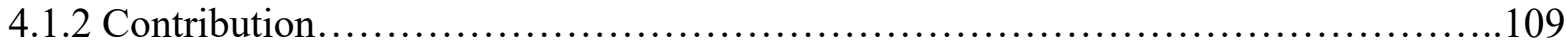

4.1.3 Abstract.................................................................. 110

4.1.4 References.........................................................................111

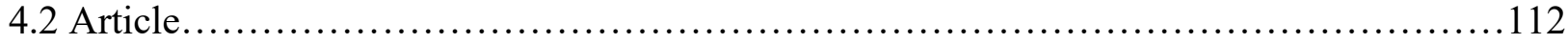

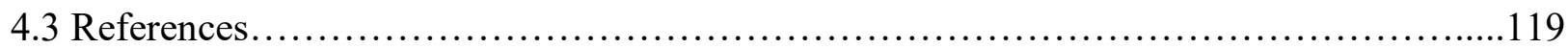

Chapter 5 Detection of Nitroaromatic Compounds by Fluorescent Conjugated

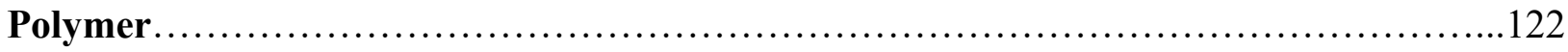

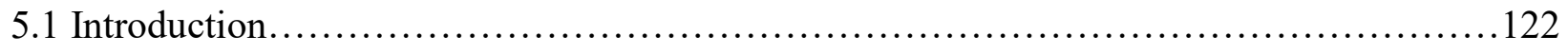

5.11 Overview of nitroaromatic compounds................................... 122

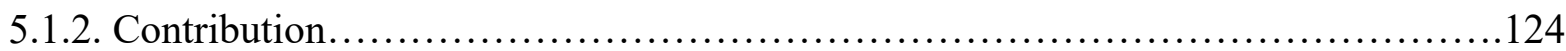

5.1.3. Abstract........................................................... 124

5.1.3 References.............................................................. 126

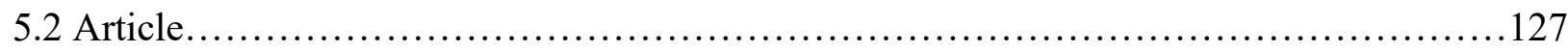




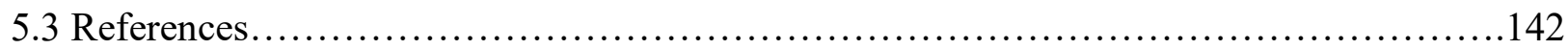

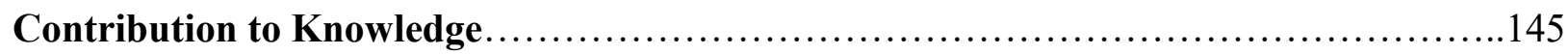

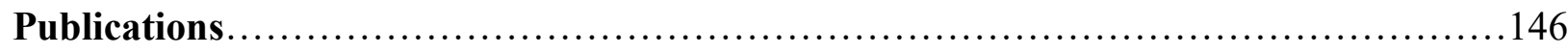

Appendices

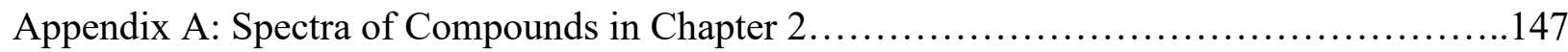

Appendix B: Spectra of Monomers and Polymers in Chapter 3........................... 161

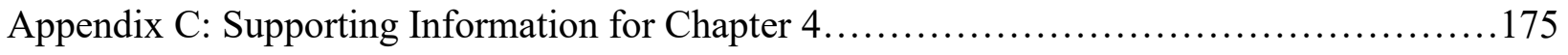

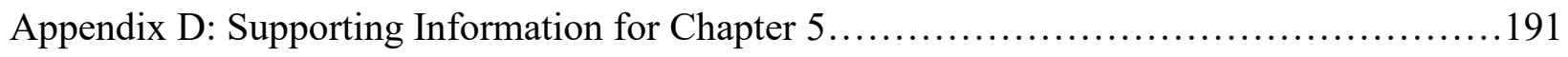




\section{List of Figures}

Figure 1.1 Structures of mono-, di- and polyisocyanates.................................

Figure 1.2 Structure of syn and anti-conformer of acyl azide...............................

Figure 1.3 Resonance structures of the isocyanate groups................................. 15

Figure 1.4 Inter-atomic binding types of isocyanate..................................... 16

Figure 1.5 Structures of selected amine reagents for derivatization of isocyanates...............19

Figure 1.6 Structures of common explosive compounds...................................21

Figure 1.7 Schematic representation of electron exchange for fluorescence quenching.........23

Figure 1.8 Aggregation caused quenching and aggregation-induced emission................24

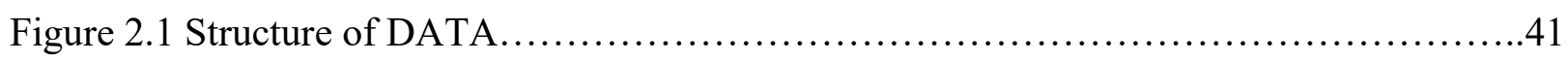

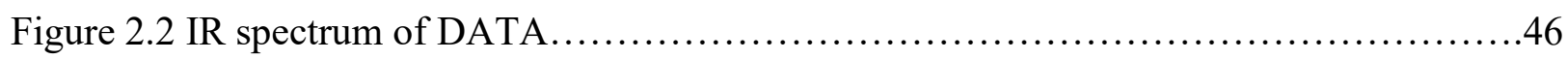

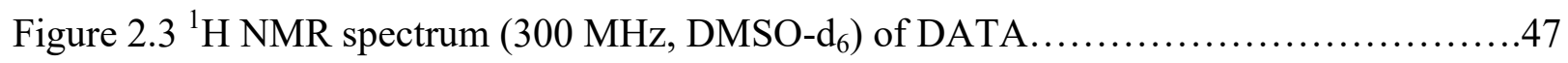

Figure $2.4{ }^{13} \mathrm{C}$ NMR spectrum $\left(75 \mathrm{MHz}\right.$, DMSO-d $\left.\mathrm{d}_{6}\right)$ of DATA ............................

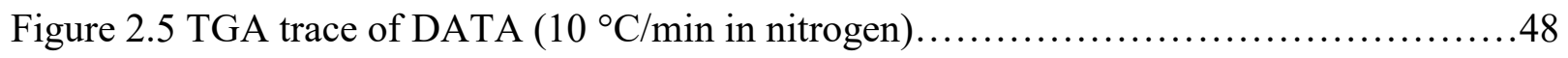

Figure 2.6 IR spectrum of DATA in dioctyl terephthalate at $150{ }^{\circ} \mathrm{C}$ after 9 minutes ..........50

Figure 2.7 IR spectrum of the reaction mixture after adding 9-heptadecanol as shown in Scheme

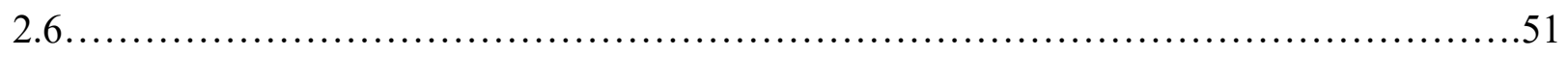

Figure 2.8 IR spectrum of dioctyl terephthalate after bubbling in $\mathrm{CO}_{2}$ at room temperature for 15 $\min$.

Figure 2.9 IR spectra of the reaction mixtures (DATA and PTMEG) taken at different time intervals. (a) 10 minutes, (b) 60 minutes, and (c) 90 minutes...............................5

Figure 2.10 IR spectrum of reaction mixture of PPDI and PTMEG.........................58

Figure 2.11 IR spectrum of the reaction mixture of DATA and PTMEG (1:1 molar ratio) at 130

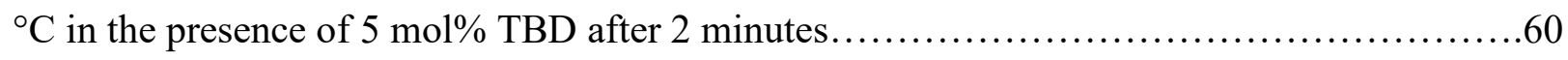


Figure 2.12 The IR spectra of reaction mixture of DATA and PTMEG (2.2:1 molar ratio) heated at $80{ }^{\circ} \mathrm{C}$ in the presence of $5 \mathrm{~mol} \% \mathrm{TBD}$ after (a) 5 minutes (b) 15 minutes.................61

Figure 2.13 IR spectrum for the in-situ conversion of prepolymers $\mathbf{P 3}$ to $\mathbf{P 1} \ldots \ldots \ldots \ldots \ldots \ldots 63$

Figure 2.14 IR spectrum of the reaction mixture after addition of BDO in 5 minutes at $80{ }^{\circ} \mathrm{C}$ to the product from the reaction of DATA and PTMEG.........................................64

Figure 3.1 Isocyanate-functionalized monomers......................................77

Figure 3.2 Methylmethacrylate, $N$-acetoxymethacrylamide and 2-hydroxyethyl methacrylate...78

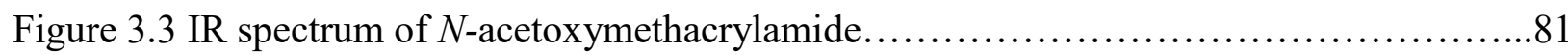

Figure $3.4{ }^{1} \mathrm{H}$ NMR spectrum (300 MHz, DMSO-d ${ }_{6}$ ) of $N$-acetoxymethacrylamide..............81

Figure 3.5 IR spectra of (a) $N$-acetoxymethacrylamide, (b-d) P5-P7 and (e) PMMA.............84

Figure $3.6{ }^{1} \mathrm{H}$ NMR spectra of (a) $N$-acetoxymethacrylamide and (b-d) P5-P7 .................85

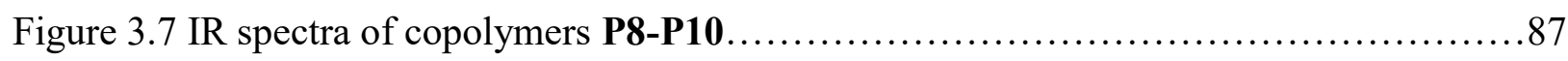

Figure 3.8 IR spectra of copolymer (a) P8 and (b) P11 ................................. 89

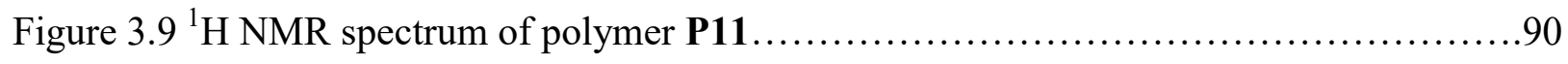

Figure 3.10 Absorption spectra of P5, P8, P11 and DR1 ..............................90

Figure 3.11 IR spectra of terpolymers (a) P12 (containing $2.5 \mathrm{~mol} \%$ of AMAA and $2.5 \mathrm{~mol} \%$ of HEMA), and (b) P13 (containing 5 mol \% of AMAA and 5 mol \% of HEMA................93 Figure $3.12{ }^{1} \mathrm{H}$ NMR spectra of (a) terpolymers P12 (containing $2.5 \mathrm{~mol} \%$ of AMAA and 2.5 mol $\%$ of HEMA) and (b) P13 (containing 5 mol \% of AMAA and 5 mol \% of HEMA)........94 Figure 3.13 Thermal conversions $\left(130^{\circ} \mathrm{C}\right)$ of acetoxyamide group in $\mathbf{P 1 2}$ to NCO group in

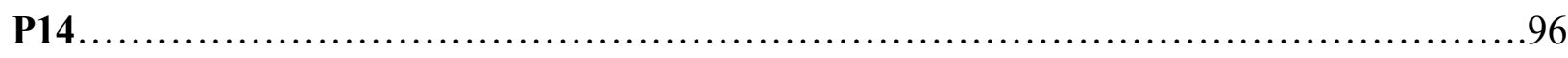

Figure 3.14 The crosslinked film of polymer P15 in chloroform (a) floating and (b) freestanding. .97 
Figure 3.15 IR spectrum of free-standing film of $\mathbf{P 1 5}$ (after heating at $140^{\circ} \mathrm{C}$ )............98

Figure 5.1 Classification of Explosives based on structure, performance, and sensitivity......122 


\section{List of Schemes}

Scheme 1.1 Synthesis of isocyanate by phosgenation of primary amine...................... 4

Scheme 1.2 General reaction of primary amine salt and phosgene.............................4

Scheme 1.3 Preparation of diphosgene and triphosgene....................................

Scheme 1.4 Isocyanate preparation from substituted anilines................................6

Scheme 1.5 Industrial preparation of MDI from nitrobenzene...............................6

Scheme 1.6 Reductive carbonylation of nitroarene to form isocyanates.......................

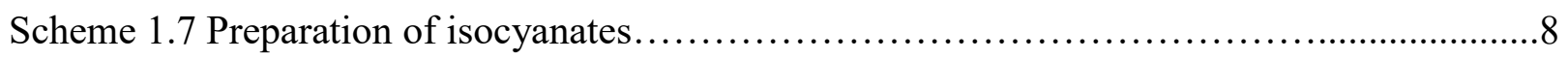

Scheme 1.8 Mechanisms for (a) thermal and (b) photo-induced Curtius rearrangement...........9

Scheme 1.9 General preparation of isocyanates by Hofmann rearrangement.....................10

Scheme 1.10 Reaction mechanism from amide to isocyanate by Hofmann rearrangement.......11

Scheme 1.11 The TBCA-mediated Hofmann rearrangement reaction.........................12

Scheme 1.12 Synthesis of methyl carbamate using solid-supported base......................12

Scheme 1.13 Synthetic route to isocyanates via Lossen rearrangement.......................13

Scheme 1.14 First reported synthesis of phenyl isocyanate by Lossen rearrangement............13

Scheme 1.15 Mechanism for the reaction of hydroxamic acid with bromodimethylsulfonium

bromide in the presence of amine...................................................... 14

Scheme 1.16 General mechanism of the Lossen rearrangement...............................15

Scheme 1.17 Common reactions of isocyanates..............................................

Scheme 2.1 Formation of NCO-terminated prepolymer and polyurethane.......................40

Scheme 2.2 Synthesis of DHTA....................................................... 43

Scheme 2.3 Synthesis of DATA........................................................... 44

Scheme 2.4 Plausible mechanism for the formation of DATA ..............................45

Scheme 2.5 Plausible mechanism for the formation of $N$-substituted DATA...................45 
Scheme 2.6 Reaction of DATA and heptadecane-9-ol. .49

Scheme 2.7 Mechanism for the reaction of DATA and heptadecane-9-ol....................50

Scheme 2.8 Reaction of DATA and heptadecane-9-ol in the presence of a base catalyst........52

Scheme 2.9 Reaction mechanism of DATA and heptadecane-9-ol in the presence of a base.....52

Scheme 2.10 Reactions of ABA with $n$-butanol under two different reaction conditions........54

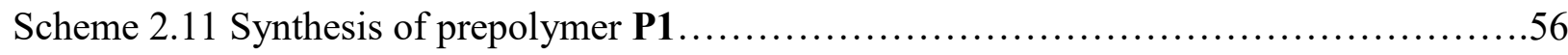

Scheme 2.12 Synthesis of NCO-terminated prepolymer with PPDI.........................58

Scheme 2.13 One-pot synthesis of polyurethane from DATA and PTMEG in the presence of a

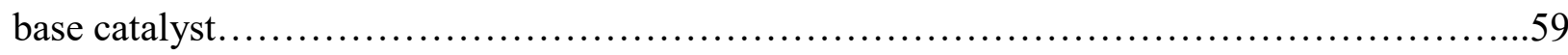

Scheme 2.14 Synthesis of AA-terminated prepolymer P3 in the presence of a base catalyst......61

Scheme 2.15 In-situ conversion of acetoxyamide-terminated prepolymer $\mathbf{P 3}$ to NCO-terminated prepolymer $\mathbf{P 1}$ and subsequent chain extension with BDO to form polyurethane $\mathbf{P 4} \ldots \ldots \ldots \ldots . .62$

Scheme 3.1 Synthesis of the pendent NCO-containing polystyrene using 4-vinylbenzoyl azide

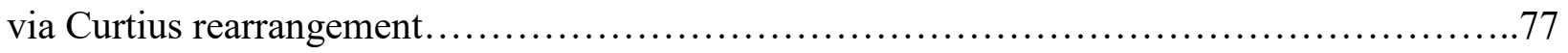

Scheme 3.2 Synthesis of $N$-hydroxymethacrylamide....................................... 79

Scheme 3.3 Synthesis of $N$-acetoxymethacrylamide ....................................... 80

Scheme 3.4 Synthesis of MMA-AMAA copolymers P5-P7 ............................. 82

Scheme 3.5 Conversion of acetoxyamide to $\mathrm{NCO}$ in copolymers at $110-130{ }^{\circ} \mathrm{C} \ldots \ldots \ldots \ldots \ldots . . . .66$

Scheme 3.6 Reaction of P8 and DR1 to yield P11..................................... 88

Scheme 3.7 Synthesis of MMA-AMAA-HEMA terpolymers P12-P13 ......................91

Scheme 3.8 Thermal conversion of terpolymer P12 to P14 ..............................95

Scheme 3.9 Heating of polymer P14 to form the crosslinked polymer P15 ....................97 


\section{List of Tables}

Table 1.1 Vapor pressures, boiling points and melting points of some explosives.............22

Table 3.1 The onset temperatures for decomposition of copolymers P5-P7 ....................83

Table 3.2 The calculated and obtained ratios of AMAA in polymers (from IR spectroscopy)....86

Table 3.3 The calculated and obtained ratios of monomers in polymers.....................94

Table 3.4 The onset temperatures for decomposition of terpolymers P12 and P13..........95 


\section{List of Symbols and Abbreviations}

\begin{tabular}{|c|c|}
\hline $\mathrm{ABA}$ & $N$-acetoxybenzamide \\
\hline ACQ & Aggregation-caused quenching \\
\hline $\mathrm{AcOH}$ & Acetic acid \\
\hline ACGIH & American Conference of Governmental Industrial Hygienists \\
\hline AIE & Aggregation-induced emission \\
\hline AMAA & $N$-acetoxymethacrylamide \\
\hline $\mathrm{BDO}$ & butanediol \\
\hline $\mathrm{CH}_{3} \mathrm{CN}$ & Acetonitrile \\
\hline $\mathrm{CV}$ & Cyclic voltammetry \\
\hline DATA & Diacetoxyterephthalamide \\
\hline DBA & $n$-Dibutylamine \\
\hline DBU & 1,8-Diazabicyclo[5.4.0]undec-7-ene \\
\hline DHTA & $N^{1}, N^{4}$-dihydroxyterephthalamide \\
\hline $\mathrm{DMF}$ & $N, N$-Dimethylformamide \\
\hline DNT & 2,4-Dinitrotoluene \\
\hline $\mathrm{DSC}$ & Differential scanning calorimetric \\
\hline FcPZ & Ferrocenoyl piperazide \\
\hline FQR & Fluorescence Quenching Response \\
\hline GPC & Gel permeation chromatography \\
\hline HDI & 1,6-Hexamethylene diisocyanate \\
\hline HEMA & 2-hydroxyethyl methacrylate \\
\hline HOMO & Highest occupied molecular orbital \\
\hline IEM & 2-isocyanatoethyl methacrylate \\
\hline
\end{tabular}




$\begin{array}{ll}\text { IPDI } & \text { Isophorone diisocyanate } \\ \text { LUMO } & \text { Lowest unoccupied molecular orbital } \\ \text { MMA } & \text { Methyl methacrylate } \\ \text { MAMA } & \text { 9-(N-methylaminomethyl)anthracene } \\ \text { MAP } & \text { 1-(9-anthracenylmethyl)piperazine } \\ \text { MeOIP } & \text { 4-Methoxyphenyl isocyanate } \\ \text { MDI } & \text { 4,4'-Methylenebis(phenyl isocyanate) } \\ \text { MMNTP } & \text { 4-methoxy-6-(4-methoxy-1-naphthyl)-1,3,5-triazine-2-(1-piperazine) } \\ \text { 2-MP } & \text { 1-(2-Methoxyphenyl)piperazine } \\ \text { NBDPZ } & \text { 4-Nitro-7-piperazino-2,1,3-benzoxadiazole } \\ \text { NO } 2 \text { PI } & \text { 4-Nitrophenyl isocyanate } \\ \text { PA } & \text { 2,4,6-Trinitrophenol } \\ \text { PAC } & \text { 9-Anthracenylmethyl 1-piperazinecarboxylate } \\ \text { PET } & \text { Photo-induced electron transfer } \\ \text { PMMA } & \text { Poly(methyl methacrylate) } \\ \text { 2-PP } & \text { 1-(2-Pyridyl)piperazine } \\ \text { ppb } & \text { parts per billion } \\ \text { Ppt } & \text { parts per trillion } \\ \text { PPDI } & \text { T-Phenylene diisocyanate } \\ \text { PIMEG } & \text { phenyl isocyanate } \\ \text { 1,5,7-Triazabicyclo[4.4.0]dec-5-ene }\end{array}$


TLC Thin layer chromatography

TGA Thermogravimetric analysis

THF Tetrahydrofuran

TMI Dimethyl meta-isopropenyl benzyl isocyanate

TNB Trinitrobenzene

TPE Tetraphenylethene

TNT 2,4,6-trinitrotoluene

TRYP Tryptamine

UV-vis Ultraviolet-visible 


\section{Chapter 1 Introduction}

\subsection{Introduction}

Isocyanates are well-known reactive compounds containing an isocyanato $(\mathrm{N}=\mathrm{C}=\mathrm{O}$ or NCO) functional group. Different types of isocyanates, such as mono-, di- and polyisocyanates depending on the number of NCO groups are available (Figure 1.1). ${ }^{1-4}$ Diisocyanates can undergo polymerization with a polyol to form NCO-terminated prepolymers or polyurethanes, depending on the ratio of diisocyanate and polyol. The NCO-end capped prepolymer is still reactive and can form polyurethane or polyurea by chain extension with a curative (e. g., diol or diamine). ${ }^{5}$

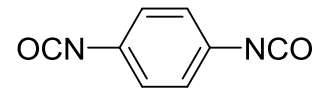

p-phenylene diisocyanate

PPDI

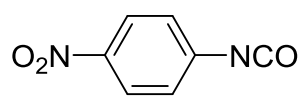

4-Nitrophenyl isocyanate

$\mathrm{NO}_{2} \mathrm{Pl}$

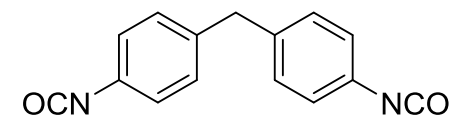

4,4'-Methylenebis(phenyl isocyanate)

MDI

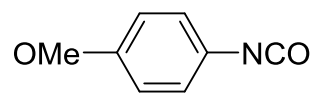

4-Methoxyphenyl isocyanate

MeOPI<smiles>Cc1ccc(N=O)cc1N=O</smiles>

Toluene 2,4-diisocyanate

TDI<smiles>O=CNCCCCCCN=O</smiles>

Hexane diisocyanate

HDI

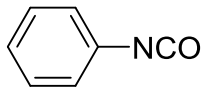

Phenyl isocyanate

PI

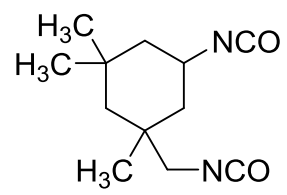

Isophorone diisocyanate IPDI<smiles>CCc1cc(C(C)c2cccc(N=O)c2)cc(C(C)(CC)c2cccc([N+](=O)[O-])c2)c1</smiles>

Polymeric MDI

Figure 1.1 Structures of mono-, di- and polyisocyanates

The discovery of isocyanates in 1849 by $\mathrm{Wurtz}^{6}$ did not lead to industrial applications until the discovery of polyurethane by Bayer in 1937. Since then, mono- and diisocyanates are 
being used in a variety of polyurethane foams, ${ }^{7-11}$ pesticides, ${ }^{12-16}$ adhesives, ${ }^{17-19}$ and coatings, ${ }^{13,20-}$ ${ }^{24}$ plastics $^{25-28}$ and so on. Usually, in foam production TDI and MDI are the two main diisocyanates, where TDI is more commonly used in North America and MDI is used in European countries. ${ }^{29}$ In addition, carbamates and ureas are the main components for fertilizers and pesticides and are prepared from isocyanates. ${ }^{30,31}$ Isocyanates are industrially demanding raw materials. The world productions for isocyanate products are several million tons per year and still increasing. ${ }^{32}$ The markets in Japan, China and India are expanding rapidly. ${ }^{33-36}$ Isocyanates have also versatile applications in organic synthesis, ${ }^{37 a}$ and can be synthesized by many methods. ${ }^{29}$ Among them, phosgenation of amine is the most widely used process in industry; whereas the Curtius, Hofmann, and Lossen rearrangements, and as well other methods remain for laboratory use. None of the methods is absolutely perfect, some have to use toxic chemicals and some are not applicable for both aliphatic and aromatic or have to use harsh reaction conditions or laborious work-up. The development or improvement of the isocyanate synthesis is still a matter of interest for researchers. ${ }^{37 b, c}$

The reactivity of isocyanates arises from its structure. They have the cumulated double bond containing functional groups $(-\mathrm{N}=\mathrm{C}=\mathrm{O})$, where the centre carbon atom has two double bonds with its adjacent two atoms and can undergo various nucleophilic reactions and selfcoupling or polymerization. ${ }^{38}$ Isocyanates are also associated with severe toxicity to human health. Various adverse health effects associated with the industrial use of isocyanates were reported ${ }^{39,40}$ and studied. ${ }^{41}$ The detection exposure limit of isocyanates are regulated at the lowest level of hazardous organic compounds in a range of 2-20 ppb and continuous reevaluation is determined on the existing detection exposure limit. ${ }^{42}$ Two different methods are used to regulate the maximal exposure limit of isocyanates. One is based on the number of total reactive 
isocyanate groups (e. g., Finland, Switzerland and UK) and the other one is based on the individual compounds (e. g., Germany and USA). ${ }^{43}$ Therefore, efficient detection of isocyanates is much needed. There are various instrument-based analytical methods reported in the literature. Among them, colorimetric and fluorometric determinations of isocyanates are widely used, which require the derivatization of isocyanates and chromatographic separation. ${ }^{44}$ Without derivatization, the method tends to give unsatisfactory results due to the sampling difficulty associated with the high reactivity of isocyanates. ${ }^{45}$

\subsection{Preparation of isocyanates}

Since a discovery of isocyanate by decomposition of a dialkyl sulfate with potassium cyanate by Wurtz in 1849, more than 25 methods for the preparation of isocyanates have been reported. ${ }^{46}$ The following are a brief summary of some important and relevant methods for isocyanate preparation.

\subsubsection{Preparation of isocyanates by phosgenation of amines}

Phosgenation of an amine or its salt is one of the most important methods for the preparation of isocyanates. The most common and industrially applied procedure is the treatment of primary amines with phosgene (Scheme 1.1) ${ }^{47}$ Hentschel in 1884 first prepared an isocyanate from the reaction of phosgene and the salt of a primary amine. ${ }^{48}$ In this method, phosgenation of a primary amine is usually carried out by dissolving it in an inert solvent before reacting with phosgene. Alternatively, by passing dry hydrogen chloride or carbon dioxide to an amine, a suspension of a salt is prepared before contacting with phosgene. ${ }^{29}$ 


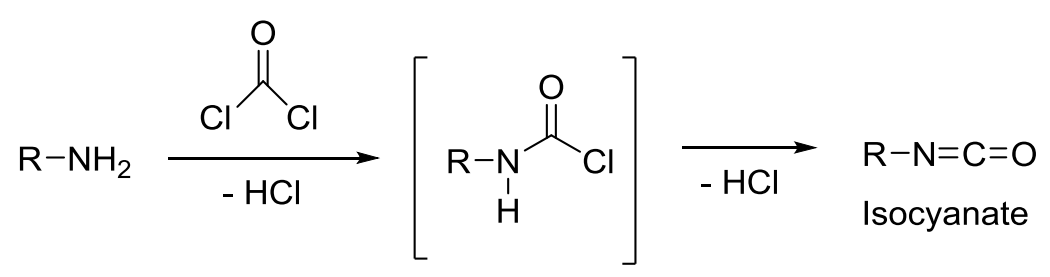

Scheme 1.1 Synthesis of isocyanate by phosgenation of primary amine

Gattermann and Schmidt did the reaction with molten methylamine hydrochloride with phosgene at $250{ }^{\circ} \mathrm{C}$ and obtained methyl carbamoyl chloride, which was decomposed by heating with lime (Scheme 1.2). They claimed an almost quantitative yield of methyl isocyanate. By applying this procedure, high-boiling isocyanates can be prepared easily in a suitable solvent. ${ }^{49}$

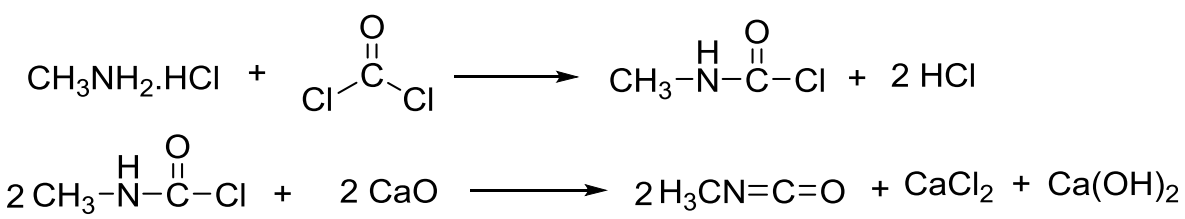

Scheme 1.2 General reaction of primary amine salt and phosgene

The main drawbacks of this method are the use of highly toxic phosgene and during the reaction two equivalents of corrosive hydrochloric acid per nitro group are generated, which requires a special plant or workroom to recycle the chlorine. Moreover, the final product is contaminated with chlorine-containing compounds ${ }^{29 b}$ and the required high reaction temperature makes it impossible or very difficult to synthesize the low-boiling products. ${ }^{46,47}$ Gaseous phosgene is hard to measure accurately and is usually used in excess. Moreover, its use and transportation are restricted and strictly controlled by the health and safety management. ${ }^{47}$

Instead of phosgene, there are two substitutes, trichloromethyl chloroformate (TCF, diphosgene) and bis(trichloromethyl) carbonate (BTC, triphosgene). TCF is a colorless liquid 
and BTC is a crystalline solid and both are soluble in common solvents like diethyl ether and chloroform. They are relatively easy to handle in the laboratory. ${ }^{50}$ Diphosgene and triphosgene are synthesized by chlorination of methyl formate and dimethyl carbonate (Scheme 1.3). One equivalent of TCF can produce two equivalents of phosgene; while BTC can make three equivalents of phosgene. The reactivity of phosgene is 18.7 times greater than that of TCF and 170 times than that of BTC. Therefore, the reactions with TCF and BTC may be controlled more easily than those with phosgene. The primary amines or their salts react with TCF or BTC and form carbamoyl chlorides, which then undergo spontaneous dehydrohalogenation and yield the corresponding isocyanates. Usually, amines are slowly added to the solution of TCF or BTC at low temperature and then the reaction temperature is increased to promote the isocyanate formation. $^{50}$

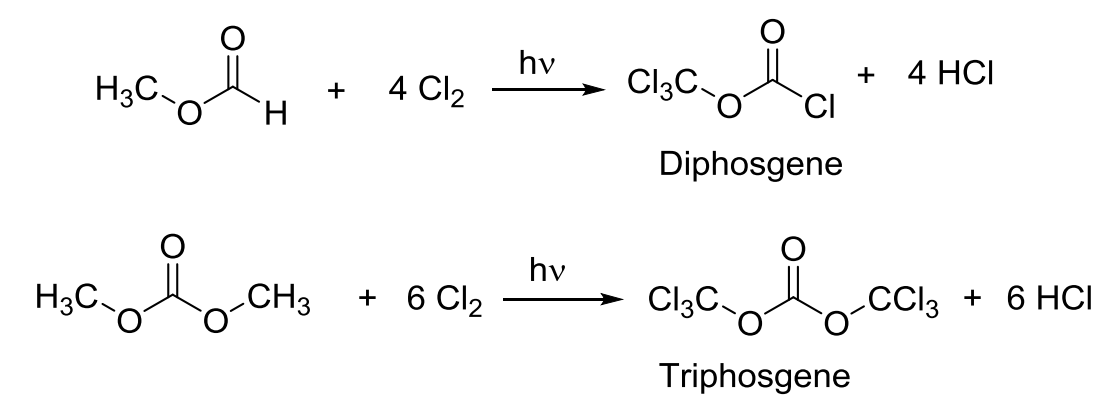

Scheme 1.3 Preparation of diphosgene and triphosgene ${ }^{50}$

Knolker et $a l .^{51}$ discovered that the reaction of substituted anilines with di-tert-butyl dicarbonate, $(\mathrm{Boc})_{2} \mathrm{O}$, in the presence of a stoichiometric amount of 4-dimethylaminopyridine in solvents like acetonitrile, dichloromethane, ethyl acetate, tetrahydrofuran and toluene at room temperature produced aryl isocyanates within $10 \mathrm{~min}$ in almost quantitative yields (Scheme 1.4). A broad range of substituted aryl and alkyl amines are used and the yields of corresponding 
isocyanates are very good. This procedure is only good for laboratory use, due to a high price of $(\mathrm{Boc})_{2} \mathrm{O} .^{47}$.

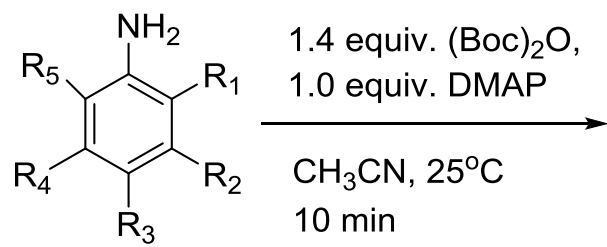<smiles>[R]Cc1c([R])c([R])c([N+]([R])=O)c([R])c1[R]</smiles>

Scheme 1.4 Isocyanate preparation from substituted anilines ${ }^{47}$

\subsubsection{Preparation of isocyanates from nitro compounds}

The aromatic isocyanates can be prepared from nitro compounds, involving the reduction of nitro compounds to amines, for example MDI from nitrobenzene (Scheme 1.5). Nitrobenzene is reduced first to aniline, which is condensed with formaldehyde and then phosgenated. ${ }^{29}$

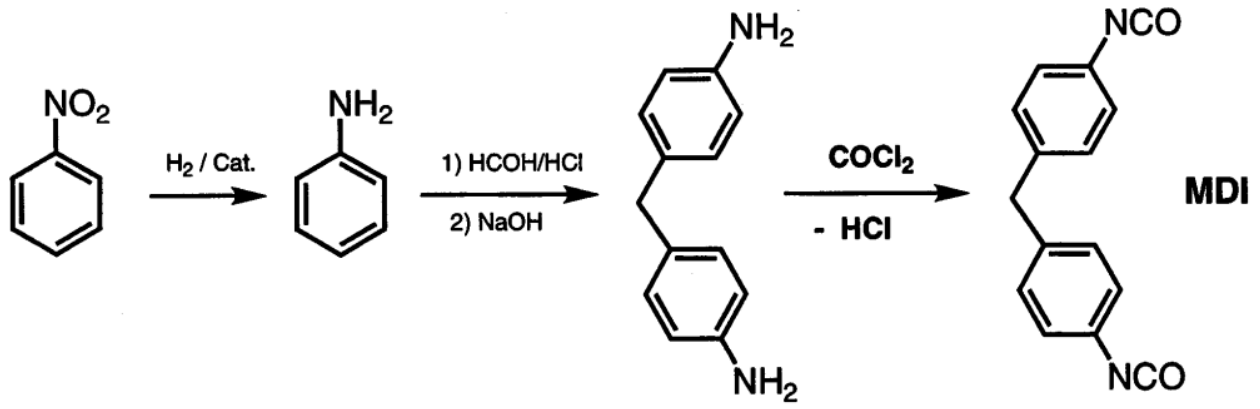

Scheme 1.5 Industrial preparation of MDI from nitrobenzene

To avoid the use of phosgene, the reductive carbonylation of nitroaromatic compounds was developed to produce isocyanates as a potentially new process to replace the phosgenation process. ${ }^{52}$ In 1967 , Hardy and Bennett first reported this reductive carbonylation reaction by CO (Scheme 1.6). ${ }^{53}$ The reaction is carried out in the presence of a palladium or rhodium catalyst 54,55 in a homogeneous or a heterogeneous medium. ${ }^{56}$ It is found that various metal oxides or chlorides $\left(\mathrm{FeCl}_{3}, \mathrm{MoCl}_{5}, \mathrm{~V}_{2} \mathrm{O}_{5}\right.$, and $\left.\mathrm{Fe}_{2} \mathrm{O}_{3}\right)$ are very effective to accelerate the reaction, although 
the effect is not well understood. Activation of the nitro group by coordination may facilitate the deoxygenation for the subsequent carbonylation. ${ }^{57}$

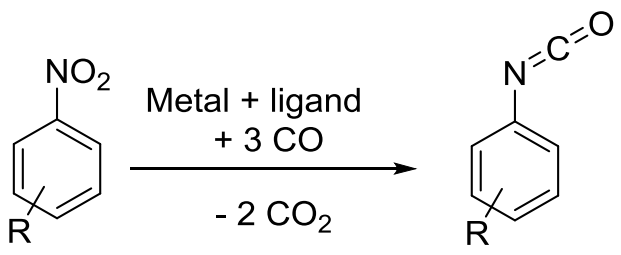

Scheme 1.6 Reductive carbonylation of nitroarene to form isocyanates

Though the catalytic carbonylation of nitroaromatic compounds is one of the most interesting alternatives for the synthesis of aromatic isocyanates from the academic and industrial points of view, the reaction does not proceed well in some cases and detectable amounts of toxic catalyst are present in the final products. ${ }^{47,58}$ Moreover, for every individual reaction the catalyst system has to be optimized first and large amounts of catalysts are used to obtain good results. ${ }^{58}$ In addition, the reaction is only applicable for the preparation of aromatic isocyanates, but commercially aliphatic isocyanates are also important for various applications in textile or paper industry. ${ }^{42}$

\subsubsection{Preparation of isocyanates by Curtius rearrangement}

The most used method in the laboratory scale besides the phosgene reaction is the Curtius rearrangement. The thermolysis of acyl azide forms the corresponding isocyanate with a loss of nitrogen gas and is known as the Curtius rearrangement, which was first described by Theodor Curtius in $1885 .^{59}$ This reaction is also known as Curtius degradation, because the product has one carbon less than the starting acyl azide. This rearrangement reaction is versatile because a diverse assortment of carboxylic acids can be converted to the corresponding acyl azides, which 
then undergo the rearrangement reaction to afford isocyanates in one pot and at moderate temperatures $\left(60-80^{\circ} \mathrm{C}\right)$ in high yields..$^{60,61}$ The resultant isocyanates are often trapped by water, alcohols or amines to afford the corresponding amines, carbamates or urea derivatives. ${ }^{62,63}$ If the thermolysis of acyl azide is conducted in absence of any nucleophiles, the isocyanate product can be isolated.

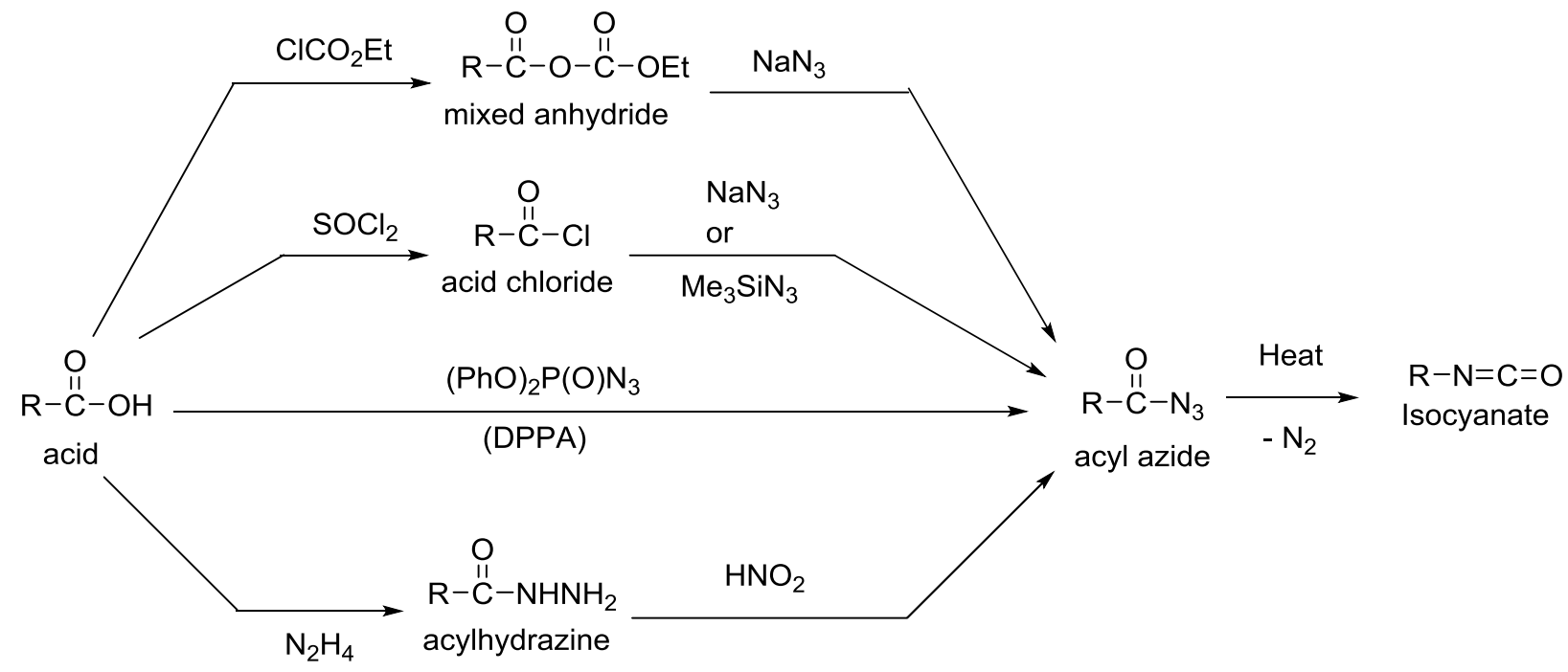

Scheme 1.7 Preparation of isocyanates

As shown in Scheme 1.7, acyl azides can be synthesized in various ways: 1) the reaction of mixed anhydrides ${ }^{64}$ or acid chlorides with alkali azide ${ }^{65}$ or trimethylsilyl azide ${ }^{66}{ }^{2}$ ) the reaction of acid with diphenyl phosphoryl azide; 3) the reaction of acylhydrazine with nitrous acid. $^{67}$ If Lewis acids are used as a catalyst, the thermolysis temperature is reduced significantly. ${ }^{68}$ Thermal rearrangement proceeds in one step with the synchronous formation of isocyanate and evolution of $\mathrm{N}_{2}$ (Scheme 1.8). ${ }^{61,69}$ On the other hand, photochemical arrangement proceeds in two steps, in which the first step is the formation of acylnitrene and the second step is the rearrangement of nitrene to isocyanate. Under the thermal conditions, the acylnitrene intermediate is very reactive and undergoes hydrogen abstraction, insertion into $\mathrm{C}-\mathrm{H}$ bonds and 
addition to $\mathrm{C}=\mathrm{C}$ bonds. $^{70,71}$ Its structure and high reactivity have been studied by theoretical calculations as well. ${ }^{72}$

a)

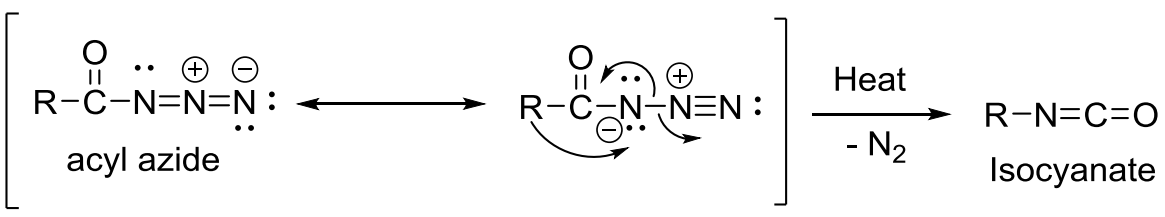

b)

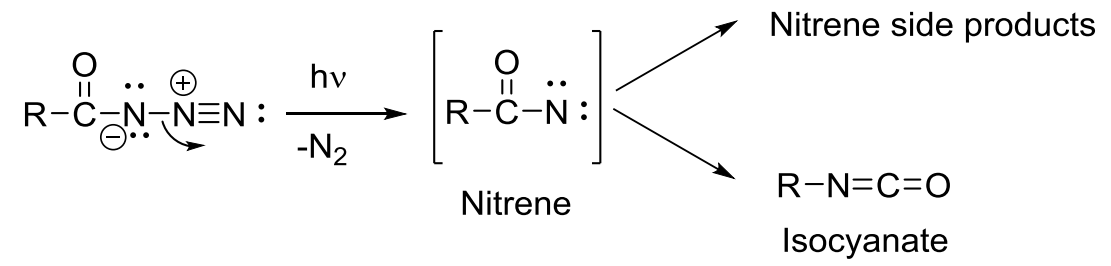

Scheme 1.8 Mechanisms for (a) thermal and (b) photo-induced Curtius rearrangement

The concerted or stepwise rearrangement involves the two (syn and anti)-conformers of acyl azide (Figure 1.2). The syn-conformer rearranges to the product in a concerted way while the other conformer rearranges in a stepwise process. Moreover, the activation energy of isomerization of syn-anti conformers is lower than those of any individual rearrangements. Thermal rearrangement almost completely proceeds via more stable syn-conformer in the concerted mechanism. Thermal rearrangement can be catalyzed by protic acids $(\mathrm{AcOH}, \mathrm{HCl}$, etc.) ${ }^{69,73}$ and Lewis acids $\left(\mathrm{BF}_{3}, \mathrm{AlCl}_{3}, \mathrm{SbCl}_{5}\right.$, etc.), ${ }^{68,74,75}$ which significantly decrease the reaction temperature and time. Interaction of Lewis acids with the acyl azide group facilitates the reaction and decreases the activation energy with a decreasing strength of Lewis acids. ${ }^{76}$

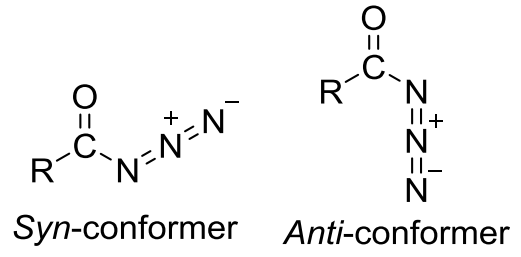

Figure 1.2 Structure of syn and anti-conformer of acyl azide 
Though the Curtius rearrangement is an efficient "phosgene-free" reaction for the preparation of isocyanates, the reaction is only suitable for laboratory use, due to the nature of toxic and explosive azides. ${ }^{61}$

\subsubsection{Preparation of isocyanates by Hofmann rearrangement}

The Hofmann rearrangement is another way to synthesize isocyanates (in situ) from primary amides. In this rearrangement, primary amides rearrange into isocyanates upon treatment with halogens and bases (Scheme 1.9). ${ }^{77,78}$ This reaction is named by discoverer August Wilhelm von Hofmann in 1881 and it is also called the Hofmann degradation because the product forms with a loss of carbon atom and the change of functional group from amide to amine. ${ }^{79}$ According to the Scheme 1.10, the reaction begins with deprotonation of the amide by a base to form an anion, which then reacts with halogen to form an $N$-haloamide. The $N$-haloamide is then deprotonated by the base and forms the corresponding unstable alkali salt which undergoes a concerted rearrangement to isocyanate via a bridged anion. ${ }^{80 a, b}$ The resulting isocyanate can be isolated under anhydrous conditions. However, the reaction is generally carried out in aqueous or alcoholic solution and the isocyanate is converted into an amine or urethane.

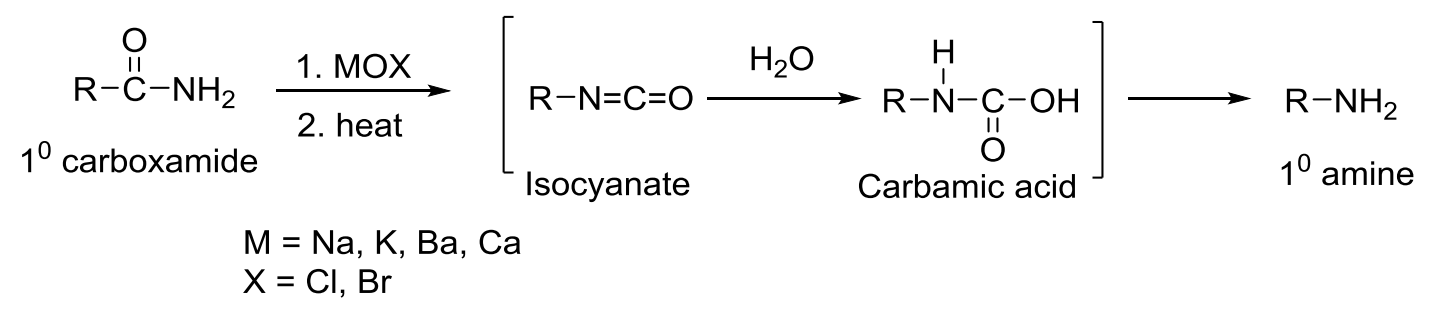

Scheme 1.9 General preparation of isocyanates by Hofmann rearrangement 


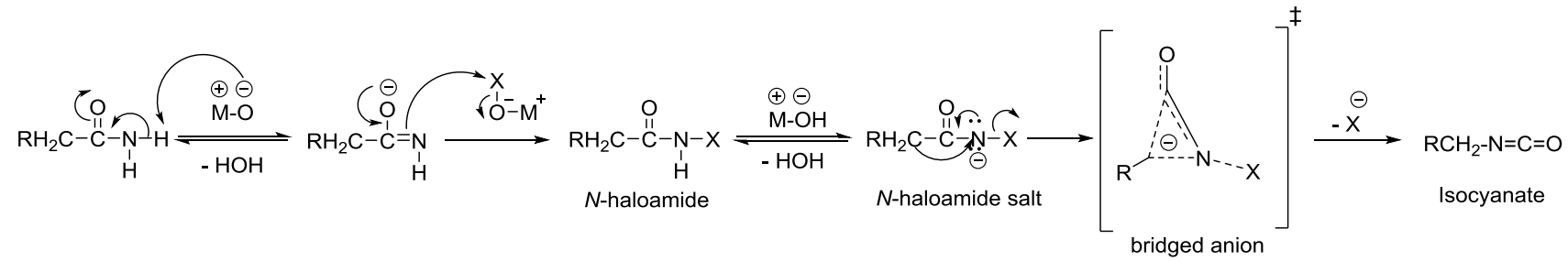

Scheme 1.10 Reaction mechanism from amide to isocyanate by Hofmann rearrangement

The use of this reaction for isocyanate preparation is limited due to the use of highly toxic bromine and strong base. ${ }^{29 b}$ Thus, modifications have been done, including the substitution of bromine with sodium hypochlorite, lead tetraacetate, $N$-bromosuccinimide and hypervalent organo-iodine compound, and the use of 1,8-diazabicyclo[5.4.0]undec-7-ene (DBU) and different solvents like DMF, $\mathrm{CH}_{3} \mathrm{CN}$, and $\mathrm{CH}_{3} \mathrm{OH}$.

In 1990, the Jew group ${ }^{81}$ reported four different types of bromonium $\left(\mathrm{Br}^{+}\right)$reagents, NBS-Hg(OAc) $)_{2}-\mathrm{R}^{\prime} \mathrm{OH}$ ， dibromantin- $\mathrm{Hg}(\mathrm{OAc})_{2}-\mathrm{R}^{\prime} \mathrm{OH} ， \mathrm{NBS}-\mathrm{AgOAc}-\mathrm{R}$ 'OH， and dibromantinAgOAc-R'OH, for the conversion of primary aliphatic and aromatic carboxamides into the corresponding carbamates. They conducted the reactions at room temperature or $45^{\circ} \mathrm{C}$ in $\mathrm{DMF}$ for 12 hours. NBS stands for $N$-bromosuccinimide and dibromantin refers to 1,3-dibromo-5,5dimethylhydantoin. Generally, the Hofmann rearrangement of higher aliphatic carboxamides is difficult and aromatic carboxamides can not be converted by using $\mathrm{C}_{6} \mathrm{H}_{5} \mathrm{I}\left(\mathrm{OCOCF}_{3}\right)_{2}{ }^{82} \mathrm{C}_{6} \mathrm{H}_{5} \mathrm{IO}^{83}$ or $\mathrm{C}_{6} \mathrm{H}_{5} \mathrm{I}(\mathrm{OTs}) \mathrm{OH} .{ }^{84}$ When using the Jew group's reported bromonium reagents, carbamates can be obtained in excellent yields from the corresponding higher aliphatic amides and aromatic carboxamides.

The Miranda group did the Hofmann rearrangement reaction of substituted benzamide to carbamate by using tribromoisocyanuric acid (TBCA) as a source of electrophilic bromine instead of NBS. ${ }^{85}$ They have shown that TBCA is very efficient for Hofmann rearrangement 
with high yields (Scheme 1.11). In the case of NBS, only $45 \%$ of its mass can be transferred in a halogenation reaction, whereas for TBCA the transferred mass would be $66 \%$. In addition, after the reaction, isocyanuric acid is the by-product and can be recovered easily and reused to produce TBCA.<smiles>[R]c1ccc(C(N)=O)cc1</smiles>

Scheme 1.11 The TBCA-mediated Hofmann rearrangement reaction

To prepare aliphatic and aromatic methyl carbamates from carboxamides via Hofmann rearrangement, $\mathrm{NaOCl}$ is used as an oxidant with a catalytic amount of $\mathrm{KF} / \mathrm{Al}_{2} \mathrm{O}_{3}(40 \% \mathrm{KF}$ in $\left.\mathrm{Al}_{2} \mathrm{O}_{3}\right)$ in $\mathrm{MeOH}$ (Scheme 1.12). ${ }^{86}$ This is the first use of the solid-supported base $\left(\mathrm{KF} / \mathrm{Al}_{2} \mathrm{O}_{3}\right)$ with inexpensive oxidant $\mathrm{NaOCl}$. $\mathrm{KOH}$ is released from $\mathrm{KF} / \mathrm{Al}_{2} \mathrm{O}_{3}$ and $\mathrm{OCl} l^{-}$as the predominant form of chlorine reacts with the amide to form $N$-chloroamide which undergoes the rearrangement to isocyanate and then methyl carbamate after subsequent reaction with methanol. This modified Hofmann rearrangement is simple and inexpensive and is applicable to a variety of aliphatic as well as aromatic amides for the synthesis of methyl carbamates. In the case of non-aqueous Hofmann rearrangements, expensive tert-butyl hypochlorite can be used. ${ }^{29 b}$ Regardless these modifications and improvements, the Hofmann rearrangement has still not been accepted by industry for the production of isocyanates.

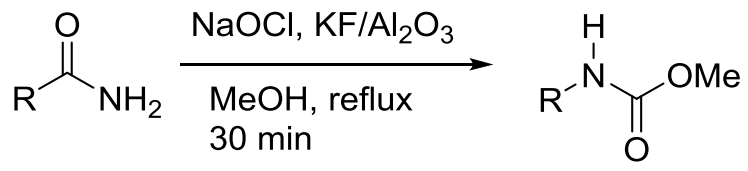

Scheme 1.12 Synthesis of methyl carbamate using solid-supported base 


\subsubsection{Preparation of isocyanates by Lossen rearrangement}

The Lossen rearrangement is another important phosgene-free method to prepare isocyanates. The starting material or hydroxamic acid is converted to the corresponding isocyanate by heating (Scheme 1.13). In comparison with other methods, the reaction condition is mild and it does not require the use of a strong base. The Lossen rearrangement reaction was first introduced by W. Lossen in 1872 for the pyrolysis of benzoyl benzohydroxamate to produce phenyl isocyanate and benzoic acid (Scheme 1.14). ${ }^{87}$

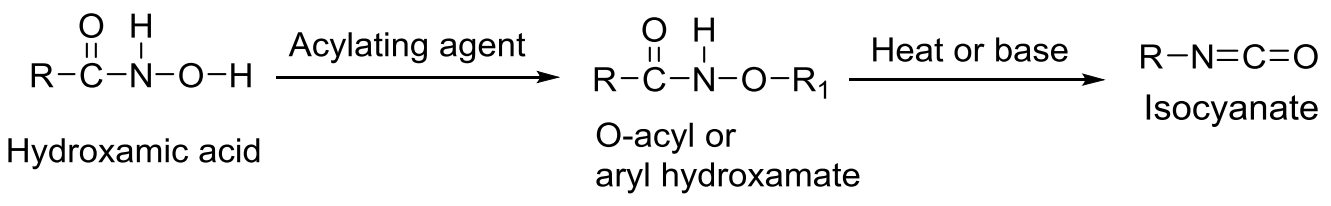

$$
\begin{aligned}
& \text { Acylating agent: anhydrides, acyl halides }\left(\mathrm{RCOCl}, \mathrm{RSO}_{2} \mathrm{Cl}, \mathrm{RPO}_{2} \mathrm{Cl}\right) \\
& \mathrm{R}_{1}=\mathrm{CO} \text {-alkyl, CO-aryl, } \mathrm{SO}_{2} \mathrm{R}, \mathrm{PO}_{2} \mathrm{R}
\end{aligned}
$$

Scheme 1.13 Synthetic route to isocyanates via Lossen rearrangement

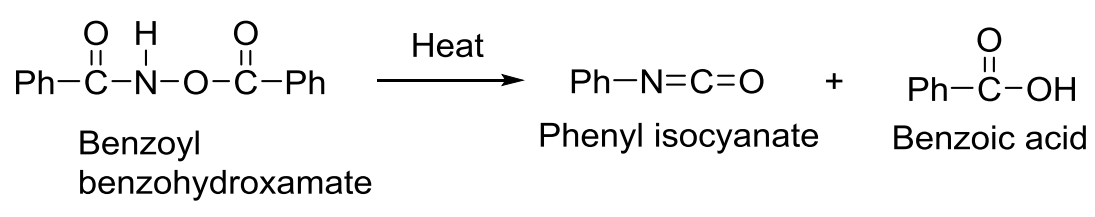

Scheme 1.14 First reported synthesis of phenyl isocyanate by Lossen rearrangement

The starting material, hydroxamic acid, is prepared easily by various methods ${ }^{88}$ It could be made from the corresponding carboxylic acid chlorides or mixed anhydrides and then reacted with hydroxylamine to form hydroxamic acid. It also could be made directly from the reaction of esters with hydroxylamine or from the reaction of aliphatic or aromatic carboxamides with hydroxylammonium chloride. Therefore, there are various scopes to make hydroxamic acids starting with any preferable starting compounds. There is no report found for the conversion of 
the free hydroxamic acid to isocyanate just by heating or in the presence of a base. ${ }^{88}$ Thus, it is necessary to activate the $N$-hydroxyl group. Anhydrides and acyl halides ${ }^{88}$ are mostly used for the $O$-acylation; and dialkylcarbodiimides ${ }^{89}$ and activated aromatic halides ${ }^{90}$ (e.g., 2,4dinitrochlorobenzene) could be used. The reaction rate of the Lossen rearrangement is directly proportional to the acidity of the leaving group. ${ }^{91}$ The Meier group synthesized methyl carbamate by in situ activation of hydroxamic acid with dimethyl carbonate in the presence of TBD or other amine bases. ${ }^{92}$ In situ activation of hydroxamic acid using bromodimethylsulfonium bromide triggers the Lossen rearrangement and the urea product is obtained in the presence of amine (Scheme 1.15). ${ }^{93}$

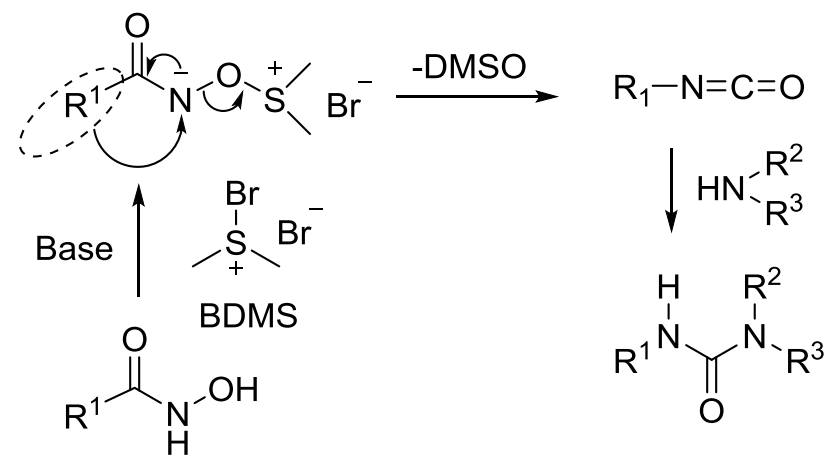

Scheme 1.15 Mechanism for the reaction of hydroxamic acid with bromodimethylsulfonium bromide in the presence of amine

The general mechanism of Lossen rearrangement is depicted in Scheme 1.16. In the first step, deprotonation occurrs at the nitrogen atom of the $O$-acyl hydroxamate by a base. The hydroxamate anion undergoes a concerted rearrangement to form isocyanate through a bridged anion. $^{94-96}$ 


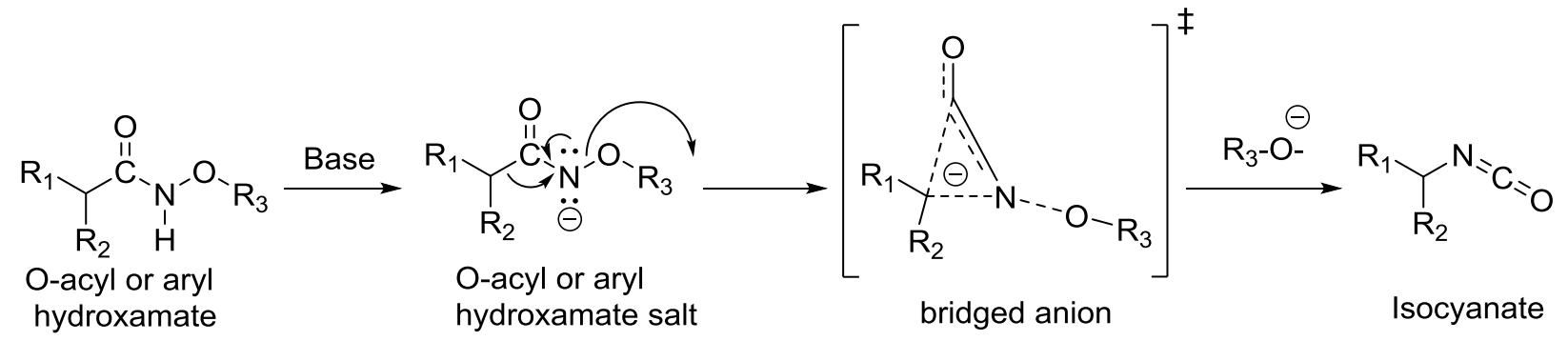

Scheme 1.16 General mechanism of the Lossen rearrangement

\subsection{Reactivity and reactions of isocyanates}

The reactivity of isocyanate is governed by the positive charge of the carbon atom in the cumulated double bond of the $\mathrm{N}=\mathrm{C}=\mathrm{O}$ group (Figure 1.3). The electron deficiency on the carbon atom is the main cause of the high reactivity of isocyanate towards nucleophilic attack and mostly the reaction occurs at the $\mathrm{C}=\mathrm{N}$ bond and only exceptionally at $\mathrm{C}=\mathrm{O}$ bond. ${ }^{97}$ When the $\mathrm{R}$ group is aromatic, then the partial negative charge on the nitrogen will be distributed throughout the benzene ring, which makes the central carbon atom more electrophilic and isocyanates become more reactive. Therefore, aromatic isocyanates are more reactive than aliphatic ones. If any electron-withdrawing group is on the aromatic ring, the isocyanate becomes more reactive towards a nucleophile. On the other hand, electron donating groups reduce the reactivity of isocyanates. $^{42}$

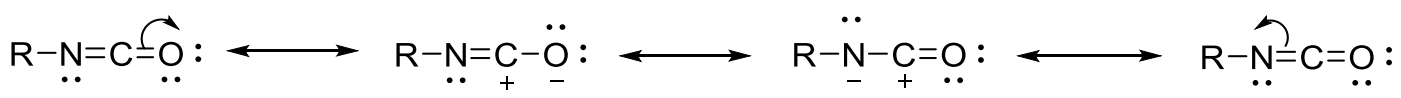

Figure 1.3 Resonance structures of the isocyanate groups.

To understand the reactivity of isocyanates, studies on the geometry and the electron density distribution of NCO moiety have been done. ${ }^{98}$ Different methods of calculations gave different results. However, it is all agreed that the minimal electron density exists on the carbon atom of $\mathrm{NCO}^{38}$ 
The reactions of particular isocyanates have been well reviewed by Ozaki, ${ }^{29 a}$ Ulrich, ${ }^{99 a}$ and Merten. ${ }^{99 \mathrm{~b}}$ The Caraculacu group divided the reactions of isocyanates into two categories on the basis of inter-atomic binding types (Figure 1.4). ${ }^{38}$ One of them is $\pi-\sigma$ transformation and the other one is $\pi-\pi$ transformation. The common reaction of isocyanate is the nucleophilic addition to the $\mathrm{N}=\mathrm{C}$ double bond, which is $\pi-\sigma$ transformation. The $\pi-\pi$ transformation is the self-addition reaction of isocyanates (Scheme 1.17).

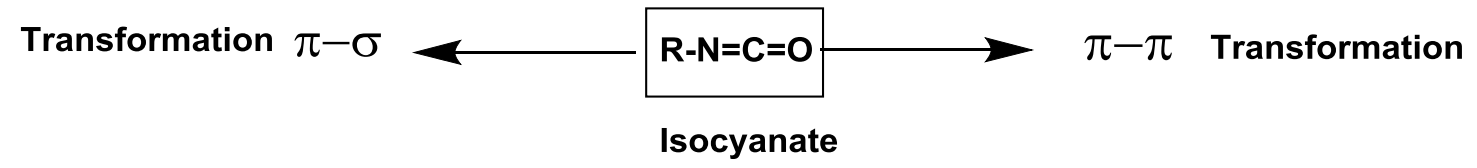

Figure 1.4 Inter-atomic binding types of isocyanate

Under acidic or basic conditions, isocyanates react with water forms unstable carbamic acid derivatives, followed by decarboxylation to primary amines. ${ }^{47}$ Isocyanates react with alcohols, amines, carboxylic acids/thioacids to produce carbamate, ${ }^{100}$ urea, ${ }^{101}$ and amide derivatives, ${ }^{102}$ respectively. In the presence of phosphanes or tertiary amines isocyanates undergo self-addition reactions form the dimer, uretidones and the trimer, isocyanurates. The isocyanurate is widely used for the formation of polyisocyanurate foams for thermal insulation. ${ }^{103}$ 


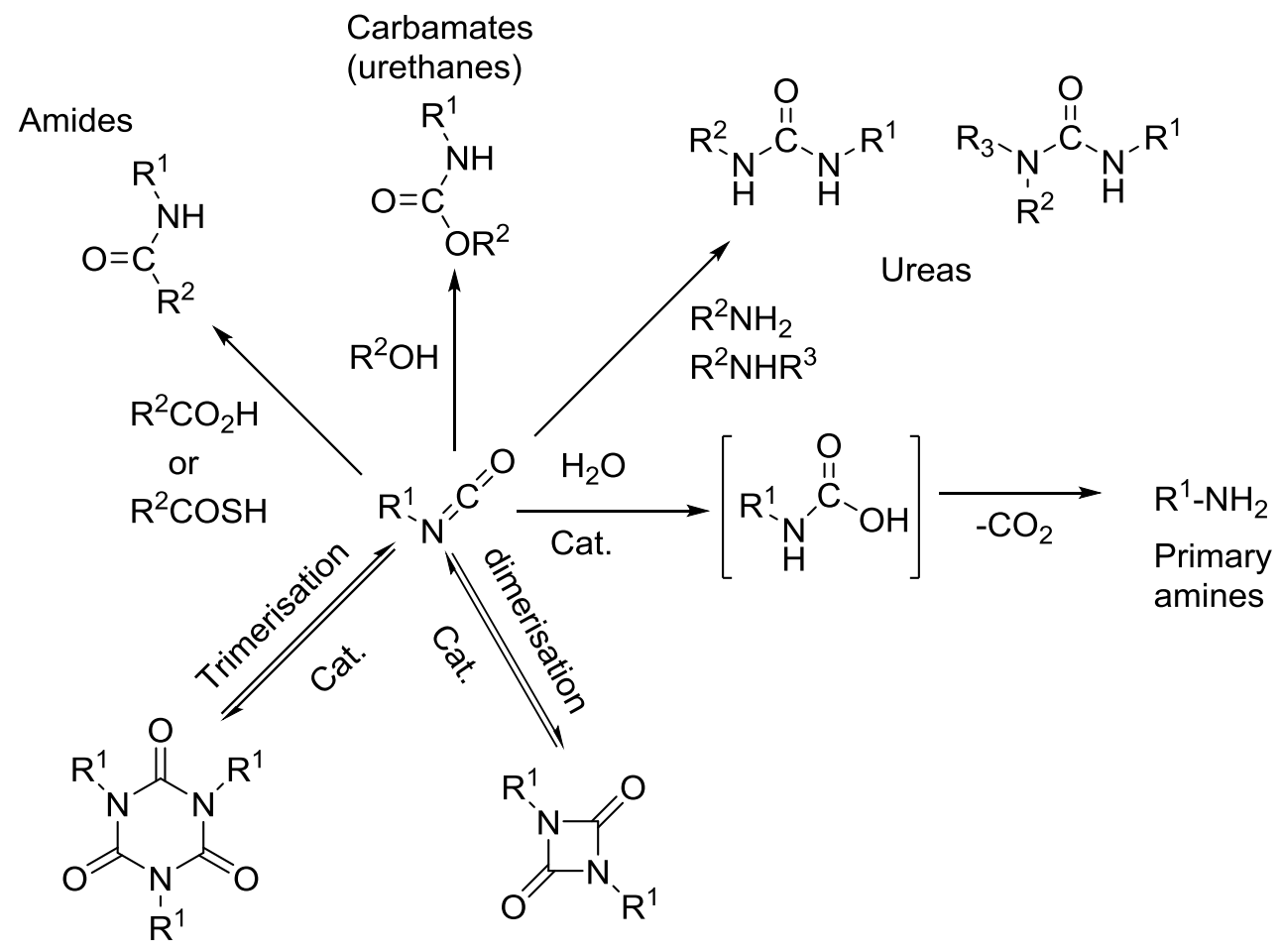

Scheme 1.17 Common reactions of isocyanates ${ }^{47}$

\subsection{Detection of isocyanates}

In order to protect workers from exposure to hazardous chemicals, the mandatory occupational exposure limits have been introduced in many countries. The exposure limits could be the average concentration of a chemical in air for normal 8 hours a day and 40 hours per week. ${ }^{104}$

In the United States, the recommended exposure limits are developed and periodically revised by the National Institute for Occupational Safety and Health (NIOSH) and then published and transmitted to the Occupational Safety and Health Administration (OSHA) for use in promulgating legal standards. Threshold limit values (TLVs) are also issued by the American Conference of Governmental Industrial Hygienists (ACGIH). 
The application of diisocyanates was first introduced by Bayer in $1937^{105}$ and from then on spread in different industries. ${ }^{42}$ Mono-isocyanates are used as intermediates for pharmaceutical and agricultural products and the long-chain aliphatic isocyanates are used for surface treatment of textile or papers. ${ }^{42}$ The most isocyanates are liquid at room temperature and volatile. In a workplace environment, workers can be exposed to isocyanate vapors during handling, spraying, heating, cutting and applying the isocyanate-containing materials. The adverse health effects from exposure to isocyanates were reported, including the studies on toxic effects of isocyanates on human health. ${ }^{39,106}$ Exposure to airborne isocyanates causes two types of physiological effects. The first one is associated with irritation of the skin, eyes, mucous membranes, and the respiratory system. The second one is associated with coughing and shortness of breath. ${ }^{107}$ Moreover, the adverse health impact associated with isocyanate exposure is asthma due to sensitization. ${ }^{108}$ In 1984, in the Indian city of Bhopal, there was an accident in a chemical plant and approximately 8000 people died within two weeks because of inhalation of methyl isocyanate. ${ }^{109}$ Therefore, workplace environment needs to be monitored and screened on a regular basis. A highly sensitive sensor is necessary to detect most isocyanates in air at the concentration of $5 \mathrm{ppb}$, as recommended in most countries. ${ }^{110}$

The determination of isocyanate based on colorimetric method was first reported by Marcali in 1956. In this method, isocyanates are hydrolyzed by acetic acid and hydrochloric acid in water to produce the amines, followed by coupling with $N$-1-naphthyl ethylenediamine to afford the azo dyes for detection. The method was successful for the detection of TDI with a detection limit of 600-130 $\mu \mathrm{g} / \mathrm{m}^{3} .111,112$ In 2000 , Wang et al. reported the fluorimetric detection of methyl isocyanate. Methyl isocyanate is absorbed in acidified dimethylsulfoxide and thus hydrolyzed to methylamine, which reacted with formaldehyde and acetyl acetone to form $\mathrm{N}$ - 
methyl-2,6-dimethyl-3,5-diacetyl-1,4-dihydropyridine for detection by fluorescence spectroscopy with a detection limit of $20 \mu \mathrm{g} / \mathrm{L} .{ }^{113}$

Isocyanates are hydrolyzed to amines by using phosphoric acid and then separated by either liquid ${ }^{114}$ or gas chromatography. ${ }^{115}$ The common analytical methods predominantly involve the derivatization with nucleophilic compounds (such as alcohols or amines), which is followed by chromatographic separation, photometric, ${ }^{116-122}$ fluorimetric ${ }^{123-1125}$ and electrochemical. ${ }^{126,127}$ Most recently used method is mass spectrometry for the detection of a trace amount of isocyanates. ${ }^{128-131} \mathrm{~A}$ recently improved method uses the proton transfer reaction, which can rapidly detect isocyanates with a high accuracy and without any sample preparation. ${ }^{132}$ The chemical structures of most commonly used derivatizing agents are presented in Figure 1.5.
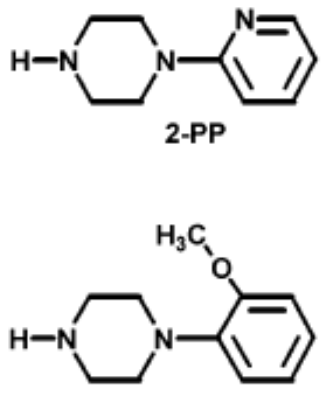

2-MP

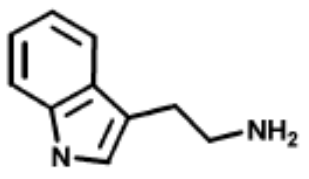

TRYP

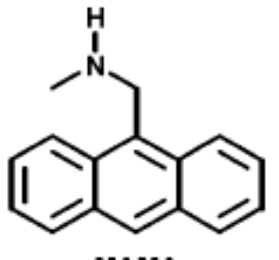

MAMA

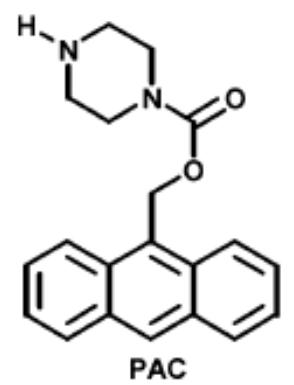<smiles>O=[N+]([O-])c1ccc(N2CCNCC2)c2nonc12</smiles>

NBDPZ<smiles></smiles><smiles>c1ccc2c(CN3CCNCC3)c3ccccc3cc2c1</smiles>

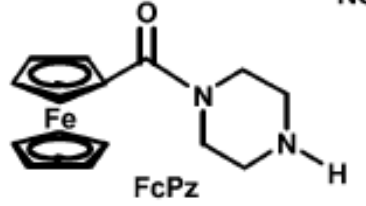

Figure 1.5 Structures of selected amine reagents for derivatization of isocyanates ${ }^{42}$ 


\subsection{Nitroaromatic explosives}

Law enforcement agencies throughout the world are faced with the challenges of detecting hidden explosives in luggage, mail, vehicles, aircraft and as well on suspects. A common explosive is 2,4,6-trinitrotoluene (TNT) and is also known to easily contaminate the ground water. ${ }^{133}$

Currently, one of the best approaches to detection of explosives is the use of the trained dogs. A trained dog is able to detect a complex sample in a range of $10 \mathrm{ppb}$ to $500 \mathrm{ppt}$ and can identify 10 different compounds. ${ }^{134}$ However, the trained dogs are expensive and their abilities rely on their conditions, training, and handler and also desire to find explosives. To improve upon using dogs and other animals for detection, the current technology utilizes the state-of-art instrumentation for detection, ${ }^{135}$ such as ion mobility spectrometry, gas chromatography coupled with mass spectrometry, ${ }^{136}$ surface enhanced Raman spectrometry, ${ }^{137,138}$ infrared spectrometry, ${ }^{139}$ energy dispersive X-ray diffraction, ${ }^{140,141}$ cyclic voltammetry, ${ }^{142-143}$ and electron capture detection. ${ }^{144}$ These methods are highly selective, extremely sensitive, robust, miniaturized, and real-time, although the instrument and equipment are expensive, need to be calibrated frequently or are difficult to handle. ${ }^{145}$

Nitroaromatics are electron deficient and have moderate vapor pressures. Based on the chemical structure, rate of decomposition and performance, explosives are classified as low and high explosives and both types are further classified into different sub-categories (see chapter 5). Low explosives burn at relatively low rates $\left(\mathrm{cm} \mathrm{s}^{-1}\right)$, whereas high explosives detonate at high velocities $\left(\mathrm{km} \mathrm{s}^{-1}\right)$. The structures and vapor pressures of common explosives are shown in Figure 1.6 and Table 1.1, respectively. These electron-deficient nitroaromatic molecules are able to form $\pi$-stacking complexes with electron-rich fluorophores, and this particular property has 
been used for their detection. ${ }^{146,147}$ TNT explosive has been studied more than other explosives. ${ }^{148} 2,4$-Dinitrotoluene (DNT) is a by-product remained in TNT, resulting from the preparation process. Even with a small amount, the significant higher vapor pressure of DNT often makes it the target molecule for detection. ${ }^{149,150}$<smiles>O=[N+]([O-])c1cc([N+](=O)[O-])c(O)c([N+](=O)[O-])c1</smiles>

PA<smiles>CN([O-])c1c([N+](=O)[O-])cc([N+](=O)[O-])cc1[N+](=O)[O-]</smiles>

Tetryl<smiles>Cc1c([N+](=O)[O-])cc([N+](=O)[O-])cc1[N+](=O)[O-]</smiles>

TNT<smiles>Cc1ccc([N+](=O)[O-])cc1[N+](=O)[O-]</smiles>

2,4-DNT<smiles>Cc1c([N+](=O)[O-])cccc1[N+](=O)[O-]</smiles>

2,6-DNT<smiles>O=[N+]([O-])c1cc([N+](=O)[O-])cc([N+](=O)[O-])c1</smiles>

TNB<smiles>O=[N+]([O-])c1cccc([N+](=O)[O-])c1</smiles>

1,3-DNB<smiles>O=[N+]([O-])c1ccc([N+](=O)[O-])cc1</smiles>

1,4-DNB NB<smiles>O=[N+]([O-])c1ccccc1</smiles><smiles>Cc1ccccc1[N+](=O)[O-]</smiles>

2-NT<smiles>Cc1ccc([N+](=O)[O-])cc1</smiles>

4-NT<smiles>Cc1cccc([N+](=O)[O-])c1</smiles>

3-NT<smiles>NC(C(=O)C(Cl)Cl)C(CO)C(O)c1ccc([N+](=O)[O-])cc1</smiles>

CIAmp<smiles>CC(C)([N+](=O)[O-])C(C)(C)[N+](=O)[O-]</smiles>

DMNB<smiles>CCC[N+](=O)[O-]</smiles>

NM NE<smiles>O=[N+]([O-])N1CN([N+](=O)[O-])CN([N+](=O)[O-])C1</smiles>

$\mathrm{RDX}$<smiles>O=[N+]([O-])N1CN([N+](=O)[O-])CN([N+](=O)[O-])CN([N+](=O)[O-])C1</smiles>

HMX<smiles>O=[N+]([O-])OCC(CO[N+](=O)[O-])(CO[N+](=O)[O-])CO[N+](=O)[O-]</smiles>

PETN<smiles>C1OCN(C23COOCN2COOC3)COO1</smiles>

HMTD

Figure 1.6 Structures of common explosive compounds ${ }^{146}$ 
Table 1.1 Vapor pressures, boiling points and melting points of some explosives (Refs. 151,152)

\begin{tabular}{|c|c|c|c|c|}
\hline Explosive & $\begin{array}{l}\text { Molecular } \\
\text { weight }\end{array}$ & $\begin{array}{l}\text { Melting } \\
\text { Point }\left({ }^{\circ} \mathrm{C}\right)\end{array}$ & $\begin{array}{l}\text { Boiling point } \\
\left({ }^{\circ} \mathrm{C}\right)\end{array}$ & $\begin{array}{l}\text { Vapor pressure at } \\
20^{\circ} \mathrm{C} \text { (Torr) }\end{array}$ \\
\hline Nitromethane & 61.0 & -29 & $100-103$ & $2.8 \times 10^{-1}$ \\
\hline 2,4-Dinitrotoluene & 182.1 & $67-70$ & $\begin{array}{l}250-300 \\
\text { (decompose) }\end{array}$ & $1.5 \times 10^{-4}$ \\
\hline 2,4,6-Trinitrotoluene & 227.1 & $80.1-81.6$ & 240 (explode) & $1.1 \times 10^{-6}$ \\
\hline 2,4,6-Trinitrobenzene & 213.1 & 122.5 & 315 & $2.2 \times 10^{-4}$ \\
\hline $\begin{array}{l}\text { Pentaerythritol } \\
\text { tetranitrate ) }\end{array}$ & 316.0 & 141.3 & $\begin{array}{l}190 \\
\text { (decompose) }\end{array}$ & $3.8 \times 10^{-10}$ \\
\hline Ethylene glycol dinitrate & 152.1 & 22 & 114 (explode) & $2.8 \times 10^{-2}$ \\
\hline Nitroglycerine & 227.0 & 13.2 & - & $2.6 \times 10^{-6}$ \\
\hline $\begin{array}{l}\text { Tetranitro- } \\
\text { triazacyclohexane }\end{array}$ & 222.3 & 204.1 & decompose & $4.1 \times 10^{-9}$ \\
\hline $\begin{array}{l}\text { Picric acid }(2,4,6- \\
\text { Trinitrophenol) }\end{array}$ & 229.1 & 122 & 300 & $5.8 \times 10^{-9}$ \\
\hline Ammonium nitrate & 80.0 & 170 & $\begin{array}{l}210 \\
\text { (decompose) }\end{array}$ & $3.3 \times 10^{-4}$ \\
\hline $\begin{array}{l}N-2,4,6 \text {-tetranitro- } N- \\
\text { methylamine }\end{array}$ & 287.1 & 129.5 & $\begin{array}{l}187 \\
\text { (decompose) }\end{array}$ & $5.7 \times 10^{-9}$ at $25^{\circ} \mathrm{C}$ \\
\hline Triacetone triperoxide & 222.1 & 98 & & $5.9 \times 10^{-2}$ at $25^{\circ} \mathrm{C}$ \\
\hline
\end{tabular}

\subsection{Fluorescence quenching}

Herein fluorescence quenching refers to the decrease in the fluorescence intensity after an analyte interacts with a receptor. A variety of molecular interactions like the excited state reaction, molecular rearrangement, energy transfer, $\pi$-stacking complex formation, ground-state complex formation and collision can quench the fluorescence. In general, the fluorescence quenching can be either dynamic or static and both in some cases. The dynamic quenching mechanism results from diffusive encounters between fluorophore and quencher in the excited state. In the static quenching process, it occurs as a result of the formation of a non-fluorescent complex in the ground state between fluorophore and quencher. ${ }^{153}$ In solution, the fluorescence quenching depends on the solvent viscosity, polarity, and $\mathrm{pH}$ of the medium. ${ }^{154}$ For electron donor-acceptor systems, usually in nonpolar solvents the mechanism of quenching is the 
formation of an excited charge-transfer complex while in polar solvents it is the simple quenching. ${ }^{155}$ Figure 1.7 shows the electron exchange or Dexter interaction, in which $\mathrm{D}_{\mathrm{E}}$ and $\mathrm{A}_{\mathrm{E}}$ represent the donor and acceptor. The excited donor has an electron in the LUMO orbital which then transfers to the LUMO of acceptor. The acceptor transfers an electron from its HOMO to donor's HOMO and the acceptor is left in the excited state. This happens when the distance between the donor and acceptor is large. In contrast, when the distance is short and in concentrated medium, the Dexter transfer can occur. In both cases, the acceptor stays in the excited state.

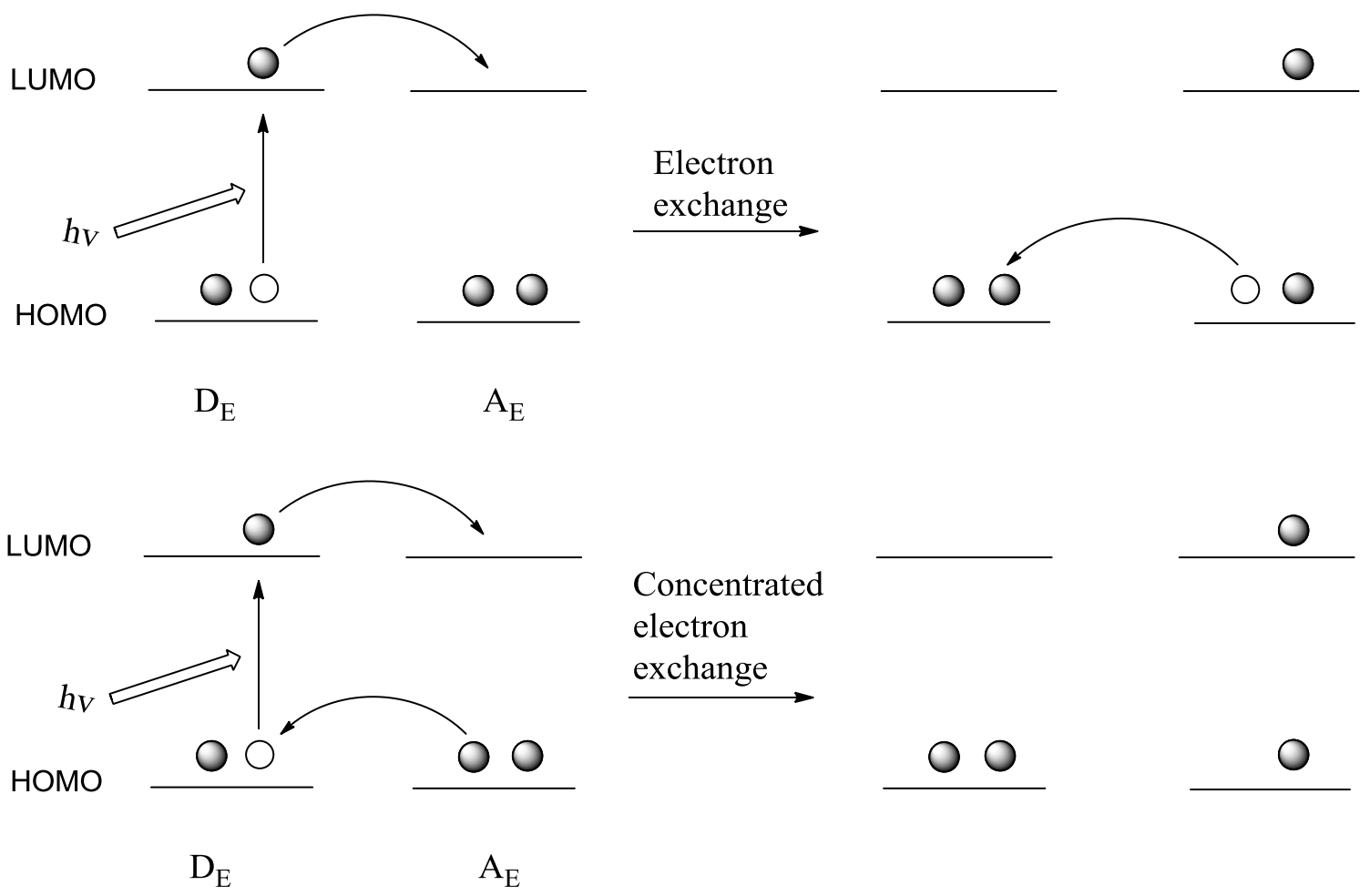

Figure 1.7 Schematic representation of electron exchange for fluorescence quenching ${ }^{155,156}$

\subsection{Aggregation-induced emission}

In a condensed state, the fluorophore molecules are located in close vicinity, which promotes the formation of exciplexes and excimers, leading to a radiationless transition. This is called the 
aggregation-caused quenching (ACQ). In 2001, Tang and his group discovered a phenomenon: a series of silole compounds were non-luminescent in solution but were emissive in the aggregated state. They named this kind of emission as "aggregation-induced emission" (AIE). ${ }^{157}$ The restriction of the active rotation of the peripheral phenyl rings (rotor) against the central core plays the vital role in the fluorescence process (Figure 1.8). In solution, the intermolecular rotation effectively annihilates the excited state thus making the solution nonemissive or weakly emissive. In aggregation or solid state the highly twisted molecular conformation inhibits $\pi-\pi$ stacking process and restricts the intramolecular rotation. The restriction of intramolecular rotation is activated by cooling, thickening, pressurization and crystallization, which blocks the nonradiative relaxation channel and populates the radiative excitons. ${ }^{158-160}$

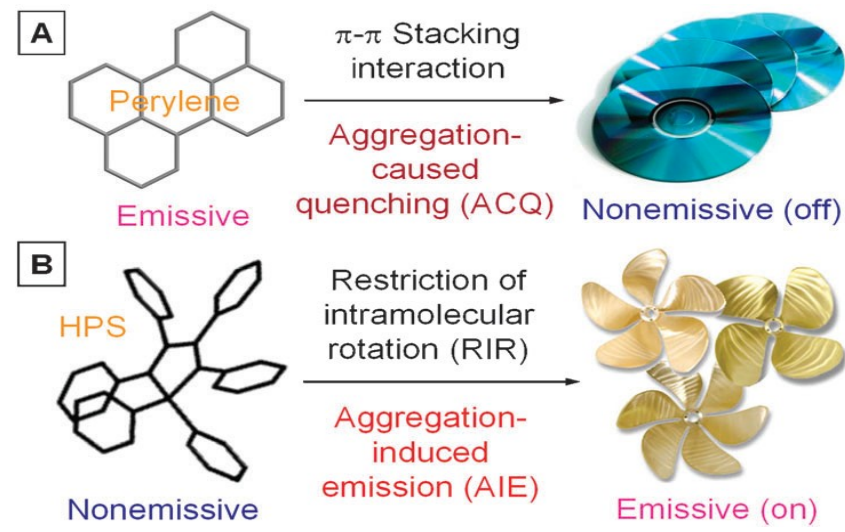

Figure 1.8 Aggregation caused quenching and aggregation-induced emission ${ }^{171}$

Many AIE compounds have been synthesized and studied. ${ }^{161-165}$ At the same time, some AIE compounds and polymers containing the AIE-enabling units such as tetraphenylethene, hexaphenyl-silole and diphenyldibenzo-fulvene have been used as chemosensors. ${ }^{166,167-169}$ Among them, tetraphenylethylene (TPE) is unique for its facile synthesis and functionalization, good stability and high fluorescence in the aggregation state. ${ }^{170}$ Recently, a TPE-containing polymer was used for the detection of nitroaromatic explosives. In the aggregation state, it is 
highly emissive or in the fluorescence "turn-on" state. After in contact with the analyte, the nonbinding electrostatic interaction between the analyte and the TPE unit causes the fluorescence quenching or "turn-off". ${ }^{167-169}$

\subsection{Rational and objectives}

Isocyanates are now in the age of over 160 years but still the attractive and important subject of research due to their high demand in the industry. The use of toxic phosgene in the production of isocyanates and adverse health effect of exposure of isocyanate call for the research and development of environmentally friendly process as well as improvement of detection methodology for isocyanates. Therefore, studies on the new routes to the preparation, reaction, and detection of industrially important isocyanates are the focal point for our research. Nitroaromatic compounds are the starting materials in the supply chain of isocyanates and considered as environmental pollutants; some are used as explosives. Therefore, detections of nitroaromatic compounds in air and water need to be studied in this thesis research.

The concept and utilization of latent isocyanates or isocyanate equivalent compounds will be explored, in order to develop a new route to the synthesis of isocyanates, NCO-terminated prepolymers, and polyurethanes. Specifically, we will study and apply the Lossen rearrangement reaction to generate the isocyanates in situ from the appropriate hydroxyamide precursors.

To develop a fluorescence method for detection of isocyanates and nitroaromatic compounds, we intend to use highly fluorescent conjugated polymers containing the propertyenhancing units of pentiptycene and tetraphenylethylene. Pentiptycene is incorporated into a conjugated polymer, in order to create a larger free volume for trapping the target molecules and to prevent $\pi$-stacking or excimer formation in the excited state in the solid film. 
Tetraphenylethylene enables the emission of conjugated polymers in the aggregated state (e.g., film), allowing for a high sensitivity in fluorescence detection.

According to the above rationale, the main objectives of this thesis research are:

1) To design and obtain a latent isocyanate precursor;

2) To synthesize the prepolymers and polyurethanes using a latent isocyanate precursor;

3) To synthesize polymethacrylates containing the pendent latent isocyanate group and study the transformation into the reactive NCO-containing polymethacrylates;

4) To develop a fluorescence method for detection of isocyanates and nitroaromatic compounds in air and water using conjugated polymers. 


\subsection{References}

1. Saunders, J. H.; Slocombe, R. J. Chem. Rev. 1948, 43, 203.

2. Arnold, R. G.; Nelsonand, J. A.; Verbanc, J. J. J. Chem. Educ. 1957, 34, 158.

3. Sato, M. J. Am. Chem. Soc. 1960, 82, 3893.

4. Kaplan, M. J. Chem. Eng.1961, 6, 272.

5. Slotta, K. H.; Tschesche, T. Chem. Ber. 1927, 60, 295.

6. Ulrich, H. Ullmann's Encyclopedia of Industrial Chemistry, VCH: New York, 1989; Vol. A14.

7. Fukuoka, S.; Chono, M.; Kohno, M. Chem. tech. 1984, 14, 670.

8. Fukuoka, S.; Chono, M.; Kohno, M. J. Chem. Soc. Chem. Commun. 1984, 6, 399.

9. Levchik, S. V.; Weil, E. D. J. Fire Sci. 2006, 24, 345.

10. Wang, J. Q.; Chow, W. K. J. Appl. Polym. Sci. 2005, 97, 366.

11. Weil, E. D.; Levchik, S. V. J. Fire Sci. 2004, 22, 183.

12. Manovyuvenskii, V. I.; Nefedov, B. K.; Khoshdurdyev, K. O. Bull. Acad. of Sci. of USSR Div. Chem. Sci. 1982, 31, 1176.

13. Metcalf, R. L. in: J.L. Kroschmitz, M. Howe-Grand (Eds.), Kirk-Othmer Encyclopedia of Chemical Technology, Wiley: New York, 1995; Vol. 14

14. Kato, T.; Suzuki, K.; Takahashi, J.; Kamoshita, K. J. Pestic. Sci. 1984, 9, 489.

15. Melinkov, N. N. Chemistry of Pesticides, Springer Verlag, Berlin, 1971.

16. Hertley, G. S. Chemicals for Pest Control, Pergamon, New York, 1969.

17. Jayabalan, M.; Lizymol, P. P. J. Polym. Mater. 2000, 17, 9.

18. Tout, R. Int. J. Adhes. Adhes. 2000, 20, 269.

19. Frisch, K. C. Polimery 1996, 41, 257. 
20. Howarth, G. A. Surf. Coat. Int. Pt. B-Coat. Trans. 2003, 86, 111.

21. Huybrechts, J.; Bruylants, P.; Vaes, A.; Marre, A. D. Prog. Org. Coat. 2000, 38, 67.

22. Howarth, G. A. JOCCA-Surf. Coat. Int. 1999, 82, 460.

23. Abate, K. JOCCA-Surf. Coat. Int. 1991, 74, 136.

24. Heath, R. J. Coat. Fab. 1985, 15, 78.

25. Zheng, Y.; Yanful, E. K.; Bassi, A. S. Crit. Rev. Biotechnol. 2005, 25, 243.

26. Maniar, K. K. Polym. Plast. Technol. Eng. 2004, 43, 427.

27. Howard, G. T. Int. Biodeterior. Biodegrad. 2002, 49, 245.

28. Antony, P.; De, S. K. J. Macromol. Sci. Polym. Rev. 2001, C41, 41

29. a) Ozaki, S. Chem. Rev. 1972, 72, 457. b) Twitchett, H. J. Chem. Soc. Rev. 1974, 3, 209.

30. Bontempi, A.; Alessio,E.; Chanos, G.; Mestroni, G. J. Mol. Catal. 1987, 42, 67.

31. Paetsch, J. in Ullmann's Encyclopaedia of Industrial Chemistry, VCH: New York, 1975, Vol. 9.

32. Randall, D.; Lee, S. (Eds.), The Polyurethanes Book, John Wiley \& Sons.

33. Fleisher, B. M. China Econ. Rev. 2006, 17, 237.

34. Kelkar, V. Econ. Polit. Week. 1999, 34, 2326.

35. Khatkhate, D. R. World Dev. 1997, 25, 1551.

36. Peng, I. Soc. Policy Adm. 2000, 34, 87.

37. a) Richter, R. H.; Priester, R. D. in: J.L. Kroschmitz, M. Howe-Grand (Eds.), KirkOthmer Encyclopedia of Chemical Technology, Wiley: New York, 1995; Vol. 14. b) Duspara, P. A.; Islam, S. M.; Lough, A. J.; Batey, R. A. J. Org. Chem. 2012, 77, 10362. c) Dube, P.; Nathel, N. F. F.; Vetelino, M.; Couturier, M.; Aboussfy, C. L.; Pichette, S.; Jorgensen, M. L.; Hardink, M. Org. Lett. 2009, 11, 5622. 
38. Caraculacu, A. A.; Coseri, S. Prog. Polym. Sci. 2001, 26, 799.

39. Fuchs, S.; Valade, P. Arch. Mal. Med. Trav. 1951, 12, 191.

40. Swensson, A.; Holmquist, C. E.; Lundgren, K. D. Br. J. Ind. Med. 1955, 12, 50.

41. a) Brugsch, H. G.; Elkins, A. N. Engl. J. Med. 1963, 268, 353. b) Vandenplas, O.; Cartier, A.; Ghezzo, H.; Cloutier, Y.; Malo, J. L. Am. Rev. Respir. Dis. 1993, 147, 1287. c) Karol, M. H.; Kramarik, J. A. Toxicol. Lett. 1996, 89,139. d) Deschamps, F.; Prevost, A.; Lavaud, F.; Kochman, S. Ann. Occup. Hyg.1998, 42, 33. e) Raulf-Heimsoth, M.; Baur, X. Am. J. Ind. Med. 1998, 34, 137. f) van Kampen, V.; Merget, R.; Baur, X. Am. J. Ind. Med. 2000, 38, 164. g) Krone, C. A.; Klingner, T. D. Pediatr. Allergy. Immunol. 2005, 16, 368. h) Diller, W. F. App. Occup. Environ. Hyg. 2002, 17, 872. i) Tarlo, S. M.; Liss, G. M. App. Occup. Environ. Hyg. 2002, 17, 902

42. Henneken, H.; Vogel, M.; Karst, U. Anal. Bioanal. Chem. 2007, 387, 219.

43. Bello, D.; Woskie, S. R.; Streicher, R. P.; Liu, Y.; Stowe, M. H.; Eisen, E. A.; Ellenbecker, M. J.; Sparer, J.; Youngs, F.; Cullen, M. R.; Redlich, C. A. Am. J. Ind. Med. 2004, 46, 480 .

44. a) Wang, H. Y.; Liu, L. D.; Ren, J. C. Analyst 1999, 124, 1327. b) Layton, R. F.; Knecht, L. A. Anal. Chem. 1971, 43, 794. c) Kubitz, K. A. Anal. Chem. 1957, 29, 814

45. a) Bianchi, A. P.; Joyner, T. K. B. J. Chromatogr. A 1997, 771, 233. b) VandenabeeleTrambouze, O.; Mion, L.; Garrelly, L.; Commeyras, A. Adv. Environ. Res. 2001, 6, 45.

46. Carnaroglio, D.; Martina1, K.; Palmisano, G.; Penoni, A.; Domini, C.; Cravotto, G. Beilstein J. Org. Chem. 2013, 9, 2378

47. Kreye, O.; Mutlu, H.; Meier, M. A. R. Green Chem. 2013, 15, 1431.

48. Hentschel, W. Chem. Ber. 1884, 17, 1284. 
49. Gattermann, J.; Schmidt, O. Lieb. Ann. 1888, 244, 29.

50. $\quad \mathrm{Su}, \mathrm{W} . ;$ Zhong, W.; Bian, G.; Shi, X.; Zhang, J. Org. Prep. Proced. Int. 2004, 36, 499.

51. Knolker, H.-J.; Braxmeier, T.; Schlechtingen, G. Angew. Chem. Int. Ed. Engl. 1995, 34, 2497.

52. Paul, F. Coord. Chem. Rev. 2000, 203, 269.

53. Hardy, W. B.; Bennett, R. P. Tetrahedron Lett. 1967, 11, 961.

54. Leconte, P.; Metz, F.; Mortreux, A.; Osborn, J. A.; Paul, F.; Petit, F.; Pillot, A. J. Chem. Soc. Chem. Commun. 1990, 22, 1616.

55. a) Elleuch, B.; Taarit, Y. B.; Basset, J. M.; Kervennal, J. Angew. Chem. Int. Ed. 1982, 21, 687. b) Weigert, F. J. J. Org. Chem. 1973, 38, 1316. c) Tietz, H.; Unverferth, K.; Schwetlik, K. Z. Chem. 1980, 20, 295. d) Tietz, H.; Unverferth, K.; Schwetlik, K. Z. Chem. 1977, 17, 368. e) Tietz, H.; Unverferth, K.; Schwetlik, K. Z. Chem. 1978, 18, 98.

f) Unverferth, K.; Hontsch, R.; Schwetlik, K. J. Prakt. Chem. 1979, 321, 86. g) Unverferth, K.; Ho“ntsch, R.; Schwetlik, K. J. Prakt. Chem. 1979, 321, 928. h) Braunstein, P.; Bender, R.; Kervennal, J. Organometallics 1982, 1, 1236. i) Gupte, S. P.; Chaudhari, R. V. J. Mol. Catal. 1986, 34, 241.

56. Cenini, S.; Ragaini, F.; Pizzotti, M.; Porta, F.; Mestroni, G.; Alessio, E. J. Mol. Catal. 1991, 64, 179.

57. Ikariya, T. Catalysis 1989, 31, 271.

58. Tafesh, A. M.; Weiguny, J. Chem. Rev. 1996, 96, 2035.

59. Buchner, E.; Curtius, T. Chem. Ber. 1885, 18, 2371.

60. Fakhr, I. M. I.; Radwan, M. A. A.; El-Batran, S.; El-Salam, O. M. E.; El-Shenawy, S. Eur. J. Med. Chem. 2009, 44, 1718. 
61. Zabalov, M. V.; Tiger, R. P. Russ. Chem. Bull. Int. Ed. 2005, 54, 2270.

62. a) Park, K. H.; Kurth, M. J. Tetrahedron Lett. 1999, 40, 5841. b) Shao, H.; Colucci, M.; Tong, S.; Zhang, H.; Castelhano, A. L. Tetrahedron Lett. 1998, 39, 7235. c) Sunami, S.; Sagara, T.; Ohkubo, M.; Morishima, H. Tetrahedron Lett. 1999, 40, 1721

63. Kaiser, C.; Weinstock, J. Org. Synth. 1988, 6, 910

64. Weinstock, J. J. Org. Chem. 1961, 26, 3511.

65. Warren, J. D.; Press, J. B. Synth. Commun. 1980, 10, 107.

66. Prakash, G. K. S.; Lyer, P. S.; Arvanaghi, M.; Olah, G. A. J. Org. Chem. 1983, 48, 3358.

67. Pozsgay, V.; Jennings, H. J. Tetrahedron Lett. 1987, 28, 5091.

68. Fahr, E.; Neumann, L. Angew. Chem. Int. Ed. 1965, 77, 591.

69. a) Newman, M. S.; Gidenhorn, H. L. J. Am. Chem. Soc. 1948, 70, 317. b) Linke, S.; Tisue, G. T.; Lwowski, W. J. Am. Chem. Soc. 1967, 89, 6308. c) Batori, S.; Messmer, A.; Timpe, H. J. Heterocycles 1991, 32, 649. c) b) Kurti, L.; Czako, B. Strategic applications of named reactions in organic synthesis; Hayhurst, J. Elsevier Academic Press: California, USA, 2005; pp 116.

70. Rauk, A.; Alewood, P. F. J. Chem. 1977, 55, 1498.

71. Goryainova,T. F.; Ershov, Yu. A.; Livshits, R. M. Khim. Vysok. Energii 1975, 9, 99 [High Energy Chem. 1975, 9 (Engl. Transl.)].

72. a) Mandel, J. L.; Hadad, C. M.; Platz, M. S. J. Org. Chem. 2004, 69, 8583. b) Zabalov, M. V.; Tiger, R. P. Russ. Chem. Bull. Int. Ed. 2007, 56, 7. c) Kakkar, R.; Zaidi, S.; Grover, R. Int. J. Quantum Chem. 2009, 109, 1058.

73. Yukawa, Y.; Tsuno, Y. J. Am. Chem. Soc. 1959, 81, 2007.

74. Coleman, R. A.; Newman, M. S.; Garrett, A. B. J. Am. Chem. Soc. 1954, 76, 4534 
75. Fahr, E.; Neumann, L. Ann. Chem. 1969, 721, 14.

76. Zabalov, M. V.; Tiger, R. P. J. Molecular Struc: Theochem. 2010, 962, 15

77. Kovacic, P.; Lowery, M. K.; Field, K. W. Chem. Rev. 1970, 70, 639.

78. Applequist, D. E.; Roberts, J. D. Chem. Rev. 1954, 54, 1065.

79. a) Loudon, G. M.; Radhakrisna, A. S.; Almond, M. R.; Blodgett, J. K.; Boutin, R. H. J. Org. Chem. 1984, 49, 4272. b) Boutin, R. H.; Loudon, G. M. J. Org. Chem. 1984, 49, 4277. c) Joshi, K. M.; Shah, K. K. J. Indian Chem. Soc. 1966, 43, 481.

80. a) Hofmann, A. W. Ber. Dtsch. Chem. Ges. 1881, 14, 2725. b) Kurti, L.; Czako, B. Strategic applications of named reactions in organic synthesis; Hayhurst, J. Elsevier Academic Press: California, USA, 2005; pp 210.

81. Jew, S.-S.; Park, H. G.; Park, H.-J.; Park, M.-S.; Cho, Y.-S. Tetrahedron lett. 1990, 31, 1559.

82. Loudon, G. M.; Radhakrishna, A. S.; Almond, M. R.; Blodgett, J. K.; Boutin, R. Ii. J. Org. Chem. 1984, 2, 4272.

83. Radhakrishana, A. S.; Rao, C. G.; Varma, R. K.; Singh, B. B.; Bhatnagar, S. P. Synthesis $1983,7,538$.

84. Lazbin, I. M.; Koser, G. Ii. J. Org. Chem. 1986, 51, 2669.

85. Miranda, L. S. M.; da Silva, T. R.; Crespo, L. T.; Esteves, P. M.; de Matos, L. F.; Diederichs, C. C.; de Souza, R. O. M. A. Tetrahedron Lett. 2011, 52, 1639.

86. Gogoi. P.; Konwar, D. Tetrahedron Lett. 2007, 48, 531.

87. Lossen, W. Liebigs Ann. Chem. 1872, 175, 271.

88. a) Yale, H. L. Chem. Rev. 1943, 32, 209. b) Bauer, L.; Exner, O. Angew. Chem., Int. Ed. 1974, 86, 419. c) Shioiri, T. Org. Synth. 1991, 6, 795. 
89. Hoare, D. G.; Olson, A.; Koshland, D. E. J. Am. Chem. Soc. 1968, 90, 1638.

90. Sheradsky, T.; Avramovici-Grisaru, S. Tetrahedron Lett. 1978, 26, 2325.

91. Swenson, J. S.; Davies,A. M.; Deyo, R. A.; Graham, B. W.; Jahn E. P.; Mattice,J. D. J Org.Chem. 1973, 38, 3956.

92. Kreye, O.; Wald, S.; Meier, M. A. R. Adv. Synth. Catal. 2013, 355, 81.

93. Yadav, D. K.; Yadav, A. K.; Srivastava , V. P.; Watal, G.; Yadav, L. D. S. Tetrahedron Lett. 2012, 53, 2890.

94. Linke, S.; Tisue, G. T.; Lwowski, W. J. Am. Chem. Soc. 1967, 89, 6308.

95. Joensson, N. A.; Moses, P. Acta Chem. Scand. B 1974, 28, 441.

96. a) Adams, G. W.; Bowie, J. H.; Hayes, R. N. J. Chem. Soc., Perkin Trans.2 1991, 2, 689.

b) Kurti, L.; Czako, B. Strategic applications of named reactions in organic synthesis; Hayhurst, J. Elsevier Academic Press: California, USA, 2005; pp 266.

97. Furunkava, J.; Yamashita, S.; Murashashi, M.; Harada, K. Macromol. Chem. 1965, 85, 80.

98. a) Sacher, E. J. Macromol. Sci. Phys. B 1976, 16, 525. b) Poppinger, D.; Radom, L.; Pople, J. A. J. Am. Chem. Soc. 1977, 99, 7806. c) Mc Lean, A. D.; Loew, G. H.; Berkowitz, D. S. J. Mol. Spectrosc. 1977, 64, 184. d) Poppinger, D.; Radom, L. J. Am. Chem. Soc. 1978, 100, 3674. e) Eyster, E. H.; Gilette, R. H. J. Am. Chem. Soc. 1940, 62, 3236.

99. a) Ulrich, H. J. Polym. Sci. Macromol. Rev. 1976, 11, 93. b) Merten, R. Angew. Chem. Int. Ed. 1971, 10, 294.

100. Chaturvedi, D. Tetrahedron, 2012, 68, 15.

101. Amendola, V.; Fabbrizzi, L.; Mosca, L. Chem. Soc. Rev. 2010, 39, 3889 
102. a) Crich D.; Sasaki, K. Org. Lett. 2009, 11, 3514. b) Sasaki K.; Crich, D. Org. Lett. 2011, $13,2256$.

103. Lehmann, P.; Malotki, P.; Tomasi, G. Eur. Patent 1924356 B1, 2008.

104. National Institute of Occupational Safety and Health, Retrieve on March 2017: https://www.cdc.gov/niosh/npg/pgintrod.html

105. Bayer, O. Angew. Chem. Int. Ed. 1947, 59, 257.

106. a) Swensson, A.; Holmquist, C. E.; Lundgren, K. D. Br. J. Ind. Med. 1955, 12, 50. b) Vandenplas, O.; Cartier, A.; Ghezzo, H.; Cloutier, Y.; Malo, J. L. Am. Rev. Respir. Dis. 1993, 147, 1287. c) Karol, M. H.; Kramarik, J. A. Toxicol. Lett. 1996, 89, 139. d) van Kampen, V.; Merget, R.; Baur, X. Am. J. Ind. Med. 2000, 38, 164. e) Tarlo, S. M.; Liss, G. M. App. Occup. Environ. Hyg. 2002, 17, 902.

107. Dhara, V. R.; Dhara, R. Arch. Environ. Health 2002, 57,391.

108. a) Malo, J.- M.; Ghezzo, H.; Élie, R. Am. J. Respir. Crit. Care. Med.1999, 159, 1879.

b) Meredith, S. K.; Bugler, J.; Clark, R. L. Occup. Environ.Med. 2000, 57, 830. c) Banks, D. E.; Butcher, B. T.; Salvagio, J. E. Ann. Allergy, 1986, 57, 389. d) Musk, A. W.; Peters, J. M.; Wegman, D. H. Am. J. Ind. Med. 1988, 13, 331. e) Vandenplas, G.; Cartier, A.; Lesage, J.; Perrault, G.; Grammer, L. C.; Malo, J. L. J. Allergy Clin. Immunol. 1988, $72,1183$.

109. a) Eckerman, I. Chemical Industry and Public Health: Bhopal as an Example, Essay in Master of Public Health, Nordic School of Public Health, Göteborg, Sweden, 2001. b) Sriramachari, S. Curr. Sci. 2004, 86, 905.

110. Gagné, S.; Lesage, J.; Ostiguy, C.; Cloutier Y.; Tra, H. V. J. Environ. Monit. 2005, 7, 145. 
111. Marcali, K. Anal. Chem., 1957, 29, 552.

112. “American Conference of Governmental Industrial Hygienists: TLVs for 1961”, Am. Ind. Hyg. Assoc. J. 1961, 22, 325.

113. Wang, H. Y.; Liu, L. D.; Ren, J. C. Analyst, 1999, 124, 1327.

114. Colli, M.; Zabarine, L.; Melzi d'Eril, G. V.; Marchetti, R. J. Chromatogr. 1993, 643, 51.

115. Melzi d'Eril, G.; Cappuccia, N.; Colli, M.; Molina, V. J. Chromatogr. A 1995, 718, 141.

116. Keller, J.; Dunlap, K. L.; Sandridge, R. L. Anal. Chem.1974, 46, 1845.

117. Dunlap, K. L.; Sandridge R. L.; Keller, J. Anal. Chem. 1976, 48, 497.

118. Tucker, S. P.; Arnold, J. E. Anal. Chem. 1982, 54, 1137.

119. Rosenberg, C.; Toumi, T. Am. Ind. Hyg. Assoc. J. 1984, 45, 112.

120. Beasley, R. K.; Warner, J. M. Anal. Chem. 1984, 56, 1604

121. Rando, R. J.; Poovey, H. G. Am. Ind. Hyg. Assoc. J. 1994, 55, 716.

122. Warwick, C. J.; Bagon, D. A.; Purnell, C. J. Analyst, 1981, 106, 676.

123. Sangö, C.; Zimerson, E. J. Liq. Chromatogr. 1980, 2, 971

124. Andersson, K.; Gudéhn, A.; Hallgren, C.; Levin, J.-O.; Nilsson, C.-A. Scand. J. Work. Environ. Health, 1983, 9, 497.

125. Tinnerberg, H.; Karlsson, D.; Dalene, M.; Skarping, G. J. Liq. Chrom. Rel. Technol. 1997, 20, 2207.

126. Streicher, R. P.; Arnold, J.E.; Ernst, M. K.; Cooper, C. V. Am. Ind. Hyg. Assoc. J. 1996, $57,905$.

127. Henriks-Eckermann, M.-L.; Välimaa, J.; Rosenberg, C. Analyst, 2000, 125, 1949.

128. Tinnerberg, H.; Spanne, M.; Dalene, M.; Skarping, G. Analyst, 1997, 122, 275.

129. Karlsson, D.; Spanne, M.; Dalene, M.; Skarping, G. Analyst, 1998,123, 117. 
130. Karlsson, D.; Dalene, M.; Skarping, G. Analyst, 1998, 123, 1507.

131. Frick-Engfeldt, M.; Zimerson, E.; Karlsson, D.; Maran, Å.; Skarping, G.; Isaksson, M.; Bruze, M. Dermatitis, 2005, 16, 130.

132. Agarwal, B.; Jürschik, S.; Sulzer, P.; Petersson, F.; Jaksch, S.; Jordan, A.; Märk, T. D. Rapid Commun. Mass Spectrom. 2012, 26, 983.

133. a) EPA 505-F-10-010, February 2010. b) EPA 505-F-14-010, January 2014.

134. Habib. M. K. Biosens. Bioelectron. 2007, 23, 1.

135. Smith, R. G.; D’souza, N.; Nicklin, S. Analyst 2008, 133, 571.

136. Hakansson, K.; Coorey, R. V.; Zubarev, R. A.; Talrose, V. L.; Hakansson, P. J. Mass Spectrom. 2000, 35, 337.

137. Kapoor, J. C.; Kannan, G. K. Defence Sci. J. 2007, 57, 571.

138. Sylvia, J. M.; Janni, J. A.; Klein, J. D.; Spencer, K. M. Anal. Chem. 2000, 72, 5834.

139. Schulte-Ladbeck, R.; Edelmann, A.; Quintás, G.; Lendl, B.; Karst, U. Anal. Chem. 2006, $78,8150$.

140. Luggar, R. D.; Farquharson, M. J.; Horrocks, J. A.; Lacey, R. J. J. X-Ray Spectrom. 1998, $27,87$.

141. Liu, P.; Qu, D.; Li, W.; Li, B.; Li, M.; Yu, D.; Liu, J. Anal. Methods, 2013, 5, 1935.

142. Zhang, H.-X.; Zhang, J.-H. Can. J. Chem. 2011, 89, 8.

143. Krausa, M.; Schorb, K. J Electroanal. Chem. 1999, 461, 10.

144. Grate, J. W.; Ewing, R. G.; Atkinson, D. A. Anal. Chem. 2010, 82, 3389.

145. Engel, Y.; Elnathan, R.; Pevzner, A.; Davidi, G.; Flaxer, E.; Patolsky, F. A. Angew. Chem., Int. Ed. Engl. 2010, 49, 6830.

146. Ostmark, H.; Wallin, S.; Ang, H. G. Propellants Explos. Phrotech. 2012, 37, 12. 
147. Davidson, W.R.; Scott, W. R.; Sleeman, R; Akery, A. K. Proc. SPIE 1994, 108, 2092.

148. Sohn, H.; Sailmor. M. J.; Magde, D.; Trogler, W. C. J. Am. Chem. Soc. 2003, 125, 3821.

149. Toal, S. J.; Trogler, W. C. J. Mater. Chem. 2006, 16, 2871.

150. a) Walsh, M. E.; Jenkins, T. F.; Thorne, P. G. J. Energetic Mater. 1995, 13, 357. b) Harper, R. J.; Almirall, J. R.; Furton, K. G. Talanta, 2005, 67, 313. c) Wu, L.; Almirall, J. R.; Furton, K. G. J. High Res. Chromatogr. 1999, 22, 279.

151. Singh, S. J. of Hazard. Mater. 2007, 144, 15.

152. Salinas, Y.; Martınez-Manez, R.; Marcos, M. D.; Sancenon, F.; Costero, A.; Parra, M.; Gil, S. Chem. Soc. Rev. 2012, 41, 1261.

153. Acemioglu, B.; Arık, M.; Efeoglu, H.; Onganer, Y. J. Mol. Struct. Teochem. 2001, 548, 165.

154. Lakowicz, J. R. Principles of Fluorescence Spectroscopy, New York, 2006, chapter 1.

155. Saha, S. K. "Fluorescence Turn-on Sensory compounds and Polymers for the Detection of Lead Ion in Water", M. Sc. Thesis, Carleton University, Ottawa, ON, Canada 2012

156. Lakowicz, J. R. Principles of Fluorescence Spectroscopy, New York, 2006, chapter 9.

157. a) Mei, J.; Leung, N. L. C.; Kwok, R. T. K.; Lam, J. W. Y.; Tang, B. Z. Chem. Rev. 2015, 115, 11718. b) Hong, Y.; Lam, J. W. Y Tang, B. Z. Chem. Soc. Rev. 2011, 40, 5361. c) Luo, J.; Xie, Z.; Lam, J. W. Y.; Cheng, L.; Chen, H.; Qie, C.; Kwok, H. S.; Zhan, X.; Liu, Y.; Zhu, D.; Tang, B. Z. Chem.Commun. 2001, 1740. d) Tang, B. Z.; Zhan, X.; Yu, G.; Lee, P. P. S.; Liu, Y.; Zhu, D. J. Mater. Chem. 2001, 11, 2974.

158. a) Liu, J.; J. W. Y.; Tang, B. Z. J. Inorg. Organomet. Polym. Mater. 2009, 19, 249. b) Wang, M.; Zhang, G.; Zhang, D.; Zhu, D.; Tang, B. Z. J. Mater. Chem. 2010, 20, 1858. 
159. a) Chen, J.; Law, C. W.; Lam, J. W. Y.; Dong, Y.; Lo, S. M. F.; Williams, I. D.; Zhu, D.; Tang, B. Z. Chem. Mater. 2003, 15, 1535. (b) Zhao, Z.; Wang, Z.; Lu, P.; Chan, C. Y. K.; Liu, D.; Lam, J. W. Y.; Sung, H. H.Y.; Williams, I. D.; Ma, Y.; Tang, B. Z. Angew. Chem., Int. Ed. 2009, 48, 7608.

160. a) Peng, Q.; Yi, Y.; Shuai, Z.; Shao, J. J. Am. Chem. Soc. 2007, 129, 9333. b) Yin, S.; Peng, Q.; Shuai, Z.; Fang, W.; Wang, Y. H.; Luo, Y. Phys. Rev. B 2006, 73, 205409.

161. Xu, B.; Chi, Z.; Li, H.; Zhang, X.; Li, X.; Liu, S.; Zhang, Y.; Xu, J. J. Phys. Chem. C, 2011, 115, 17574.

162. Lee, W.-E.; Lee, C.-L.; Sakaguchi, T.; Fujikid, M.; Kwak. G. Chem. Commun. 2011, 47 3526.

163. Zhou, X.; Li, H.-Y.; Chi, Z.-G.; Xu, B.-J.; Zhang, X.-Q.; Zhang, Y.; Liu, S.-W.; Xu, J.-R. J. Fluoresc. 2012, 2, 565.

164. Chen, J.; Law, C. C. W.; Lam, J. W. Y.; Dong, Y.; Lo, S. M. F.; Williams, I. D.; D. Zhu, D.; Tang, B. Z. Chem. Mater. 2003, 15, 1535.

165. Li, H.; Zhang, X.; Chi, Z.; Xu, B.; Zhou, W.; Liu, S.; Zhang, Y.; Xu, J. Org. Lett. 2011, 13,556 .

166. Wu, W.; Ye, S.; Huang, L.; Xiao, L.; Fu, Y.; Huang, Q.; Yu, G.; Liu, Y.; Qin, J.; Lia, Q.; Li, Z. J. Mater. Chem. 2012, 22, 6374.

167. Wu, W.; Ye, S.; Huang, L.; Xiao, L.; Fu, Y.; Qi Huang, Q.; Yu, G.; Liu, Y.; Qin, J.; Lia, Q.; Li. Z. J. Mater. Chem. 2012, 22, 6374.

168. Hu, R.; Maldonado, J. L.; Rodriguez, M.; Deng, C.; Jim, C. K. W.; Lam, J. W. Y.; Yuen, M. M. F.; Ramos-Ortiz, G.; Tang, B. Z. J. Mater. Chem. 2012, 22, 232. 
169. Liu, Y.; Deng, C.; Tang, L.; Qin, A.; Hu, R.; Sun, J. Z.; Tang, B. Z. J. Am. Chem. Soc. 2011, 133, 660 .

170. a) Hong, Y.; Haussler, M.; Lam, J.; Li, Z.; Sin, K.; Dong, Y.; Tong, H.; J. Liu, J.; Qin, A.; Renneberg, R.; Tang, B. Z. Chem.-Eur. J. 2008, 14, 6428. b) Wang, J.; Mei, J.; Yuan, W. Z.; Lu, P.; Qin, A. J.; Sun, J. Z.; Ma, Y. G.; Tang, B. Z. J. Mater. Chem. 2011, 21, 4056. c) Huang, J.; Yang, X.; Wang, J.; Zhong, C.; Wang, L.; Qin, J.; Li, Z. J. Mater. Chem. 2012, 22, 2478

171. Hong, Y.; Lam, J. W.Y. Tang, B. Z. Chem. Soc. Rev. 2011, 40, 5361. 


\section{Chapter 2 Synthesis and Characterizations of NCO-Terminated and $\mathrm{N}$ - Acetoxyamide-Terminated Prepolymers}

\subsection{Introduction}

The urethane linkage (-NH-COO-) in polyurethane (PU) is a result of the reaction between an isocyanato $(\mathrm{NCO})$ group of isocyanate and a hydroxyl $(\mathrm{OH})$ group of polyol. When a stoichiometric excess of NCO is used, an NCO-terminated prepolymer is formed, which then reacts with chain extender to yield PU (Scheme 2.1). ${ }^{1}$ This is a two-step procedure for the formation of PU. The physical characteristics (e.g., hardness) of PU products depend on the ratio of $\mathrm{NCO}$ to $\mathrm{OH}$. The prepolymers without any residual isocyanate monomers have a long shelf life and are environmentally friendly due to low volatility in comparison with isocyanate monomers.

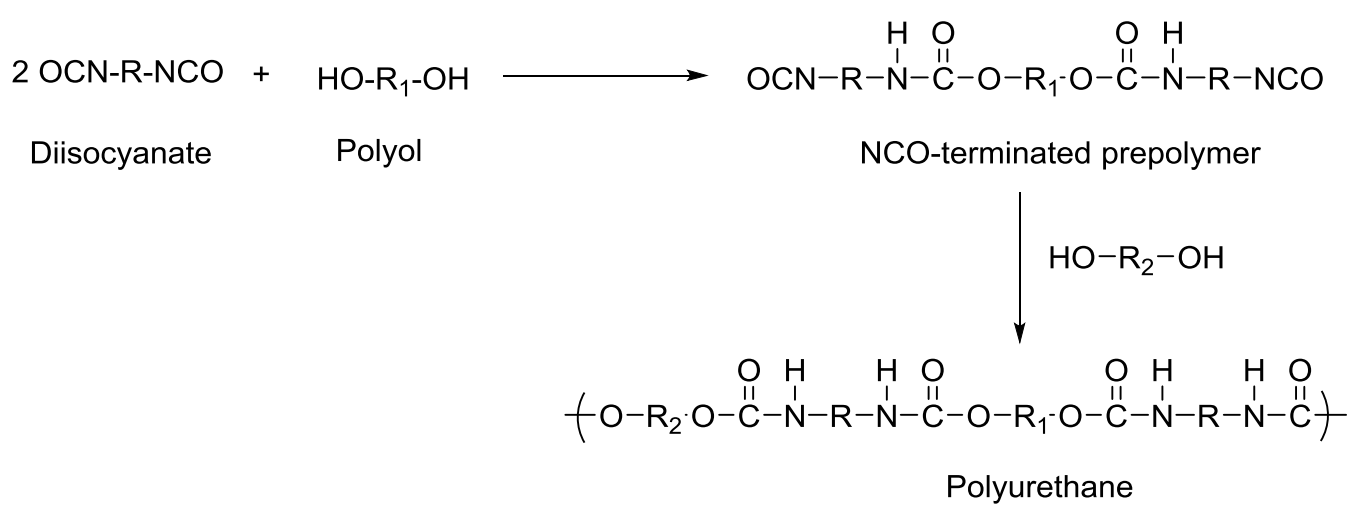

Scheme 2.1 Formation of NCO-terminated prepolymer and polyurethane

Several groups have studied NCO-terminated prepolymers from different points of view. Most research has focused on the formation of side products, the effect of time and temperature on molecular weight and removal of residual isocyanates from prepolymers. ${ }^{1-6}$ Schubert and coworkers investigated the formation of prepolymers and their side products during the step-growth 
polymerization by gel permeation chromatography (GPC), NMR spectroscopy and MALDI-TOF mass spectrometry. ${ }^{2}$ The Gogai group synthesized NCO-terminated prepolymer by the reaction of an excess of methylene diphenyl diisocyanate (MDI) with poly(propylene glycol) at $60{ }^{\circ} \mathrm{C}$ in the presence of $2,2^{2}$-dimorpholinodiethyl ether as a catalyst. ${ }^{1}$ It was found that the viscosity and weight-average molecular weight of the prepolymer increased with prolonging reaction times. Smith, et al. applied different methods to reduce the residual MDI, including wiped film evaporation, solvent-aided distillation/co-distillation, molecular sieves, and solvent extraction. ${ }^{3}$

In the Lossen rearrangement reaction, a hydroxamic acid is converted to an isocyanate intermediate that can be captured by an alcohol to form a urethane. On the contrary, the Curtius and Hofmann rearrangements use highly toxic and explosive azides or toxic bromine and produce large amounts of waste. ${ }^{4}$ Due to the advantages of the Lossen rearrangement, we were interested in its use for the formation of NCO-terminated prepolymers.

In this chapter, the synthesis of prepolymers from $N^{l}, N^{4}$-diacetoxyterephthalamide (DATA) and poly(tetramethylene ether glycol) (PTMEG) via the Lossen rearrangement is described, where an $\mathrm{N}$-acetoxyamide group (CO-NH-COO- $\mathrm{CH}_{3}$ ) (denoted as AA in this chapter) is equivalent to a latent NCO group (Figure 2.1). DATA is synthesized from the reaction of $N^{l}, N^{4}$-dihydroxyterephthalamide (DHTA) and acetic anhydride. In literature, the type of compound like DHTA is often called hydroxamic acid and the corresponding acetyl derivative is called acetohydroxamate. $^{7}$

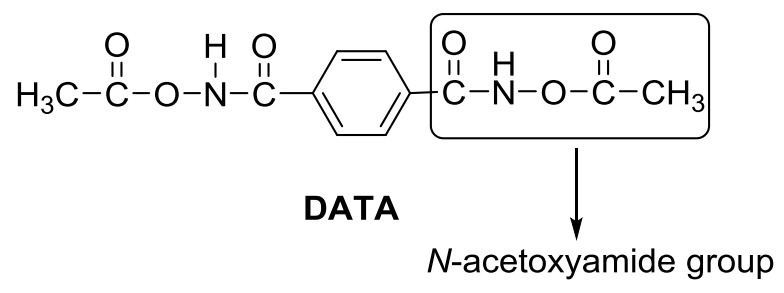

Figure 2.1 Structure of DATA 
In our study, both NCO- and AA-terminated prepolymers were targeted in the synthesis using two different reaction conditions. First, an excess of DATA reacts with PTMEG at the elevated temperatures to effect the in situ Lossen reaction and form the NCO-terminated prepolymers. In the second reaction protocol, a base catalyst is used to synthesize AA-terminated prepolymers at low temperatures. The reaction time and temperature were investigated for the synthesis of these prepolymers, mainly using infrared spectroscopy (IR).

In industry, TDI and MDI are used for making a majority of aromatic polyurethanes. PU materials derived from $p$-phenylene diisocyanate (PPDI) are produced in a relatively small quantity but perform better in many applications than the MDI and TDI-based PU materials. ${ }^{89}$ In our study, DATA is considered as a latent of PPDI for use in the formation of either latent or actual PPDI-terminated prepolymers.

\subsection{Synthesis and characterizations of $N^{l}, N^{4}$-dihydroxyterephthalamide}

The synthesis of DATA starts with DHTA. A common method for the synthesis of DHTA is the reaction of carboxylic acids or derivatives (halides, anhydrides, and esters) with hydroxylamine in the presence of a base. ${ }^{10}$ Another approach is the direct oxidation of amide, from $\mathrm{N}-\mathrm{H}$ to $\mathrm{N}-\mathrm{OH} .{ }^{11}$ The latter method requires an amide. If the parent amide is not available, then the overall process may increase the number of synthetic steps. Yamaye et al. reported the synthesis of DHTA from the reaction of poly(butylene terephthalate) with zinc acetate over 245 ${ }^{\circ} \mathrm{C}$, followed by reaction of hydroxylamine hydrochloride in a basic medium. ${ }^{12}$

Our synthetic route to DHTA is shown in Scheme 2.2 and is synthesized under basic conditions by a nucleophilic substitution reaction of diethyl terephthalate with hydroxylamine. To prepare free hydroxylamine from hydroxylamine hydrochloride, a stoichiometric amount of base solution was added to the reaction mixture. To prevent the hydrolysis of the ester group in 
diethyl terephthalate, the base solution and hydroxylamine hydrochloride were added into the reaction system at $0-5{ }^{\circ} \mathrm{C}$. The reaction continued for 48 hours and after work up DHTA was obtained as white solid. DHTA decomposes at $245{ }^{\circ} \mathrm{C}^{12}$ and is fully soluble in DMSO at room temperature and insoluble in methanol, ethanol, acetic acid and water at ambient temperatures. Although the synthesis is known, ${ }^{10,11}$ the modified procedure is given in the experimental section.

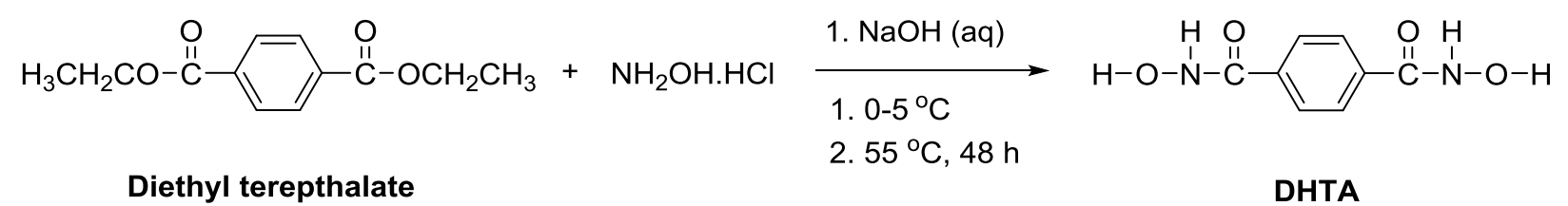

Scheme 2.2 Synthesis of DHTA

DHTA was characterized by IR, ${ }^{1} \mathrm{H}$ NMR, ${ }^{13} \mathrm{C}$ NMR and mass spectrometry. All the spectra are given in Appendix A. IR spectrum (Figure S2.1) of DHTA displays the characteristic $\mathrm{HN}-\mathrm{C}=\mathbf{O}$ band at $1642 \mathrm{~cm}^{-1}, \mathbf{H N}-\mathrm{C}=\mathrm{O}$ bending and $\mathrm{C}-\mathrm{N}$ stretching band at $1565 \mathrm{~cm}^{-1}$. The peak at $3286 \mathrm{~cm}^{-1}$ is a stretching band for NH and $\mathrm{OH}$. The ${ }^{1} \mathrm{H}$ NMR spectrum (Figure S2.2) of DHTA shows singlet peaks at 7.81, 9.14 and $11.37 \mathrm{ppm}$, which are assigned to aromatic, $\mathrm{OH}$ and $\mathrm{NH}$ protons, respectively. The ${ }^{13} \mathrm{C}$ NMR spectrum (Figure S2.3) of DHTA shows peaks at 163.8, 135.49 and $127.37 \mathrm{ppm}$, for the amide $\mathrm{C}=\mathrm{O}$, substituted aromatic carbon and aromatic carbon, respectively. High-resolution mass spectrum (Figure S2.4) shows a molecular ion peak at $\mathrm{m} / \mathrm{z}$ 196.04625, which corresponds to the desired compound.

\subsection{Synthesis and Characterizations of $N^{1}, N^{4}$-diacetoxyterephthalamide}

The synthesis of $N^{l}, N^{4}$-diacetoxytetrephthalamide (DATA) was not well established yet prior to our study. In 1970, Hynes prepared DATA from $N^{l}, N^{4}$-dihydroxytetrephthalamide 
(DHTA), acetic acid and acetic anhydride. ${ }^{13}$ Recently, DATA was synthesized by Zhang et.al following the same procedure. ${ }^{14}$ The reaction of the hydroxamic acid and acid anhydrides produced a mixture of compounds and the optimization of the reaction conditions was not discussed by Zhang or Hynes. In our research, we optimized the reaction conditions for the synthesis of DATA (see experimental section).

DATA was synthesized according to Scheme 2.3. $N, N$-diisopropylethylamine (DIPEA) remove O-H protons from DHTA to generate a nucleophile (Scheme 2.4), which undergoes the nucleophilic substitution reaction with acetic anhydride and gives the desired compound (DATA). The $\mathrm{p} k_{\mathrm{a}}$ value of the conjugate acid of DIPEA is 12 , whereas the $\mathrm{p} k_{\mathrm{a}}$ value for $\mathrm{OH}$ of a hydroxamic acid is 8.8 . The difference in the $k_{\mathrm{a}}$ values allows for the deprotonation of DHTA and formation of the nucleophile. ${ }^{15}$ In the presence of excess DIPEA, further acetylation on DATA takes place to yield an $N$-substituted by-product (Scheme 2.5). The acylation on NH was prevented by using only 2.05 equivalents of DIPEA. After 2 hours at $80{ }^{\circ} \mathrm{C}$, the reaction stopped and DATA was isolated in good yield. DATA decomposes at $150{ }^{\circ} \mathrm{C}$ and is highly soluble in DMSO at room temperature and partially soluble in methanol and ethanol at elevated temperatures.

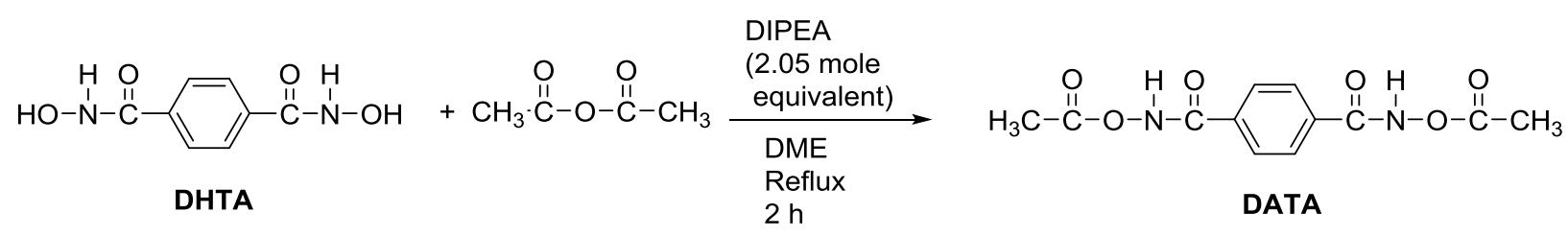

Scheme 2.3 Synthesis of DATA 


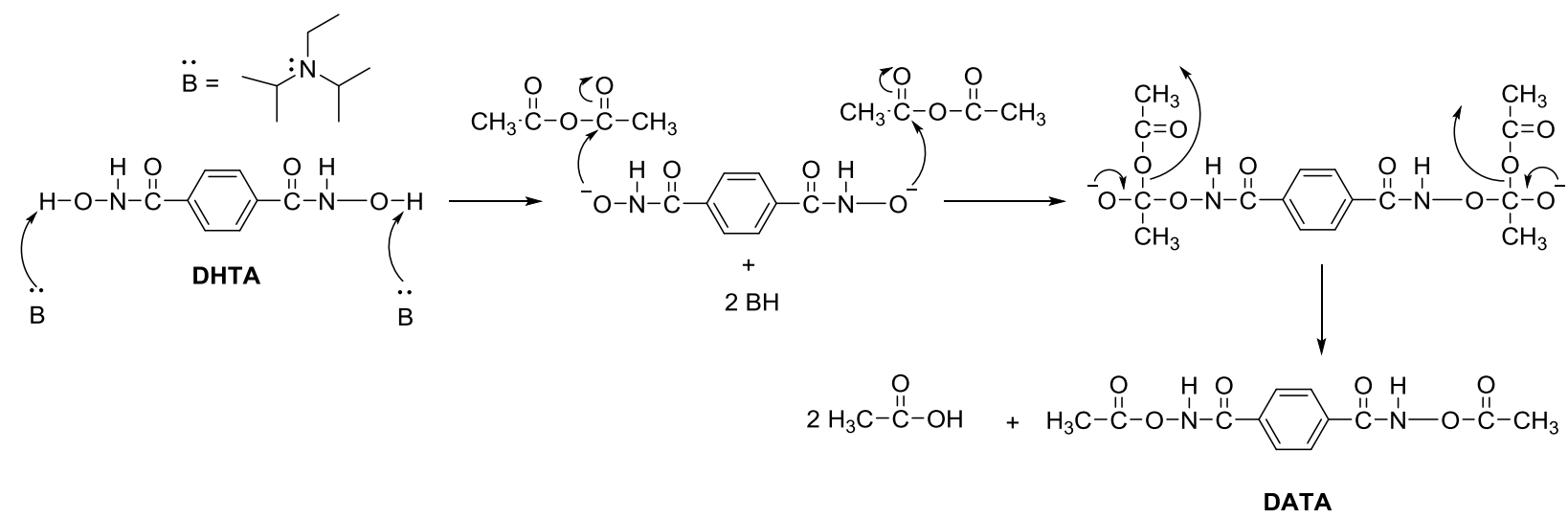

Scheme 2.4 Plausible mechanism for the formation of DATA

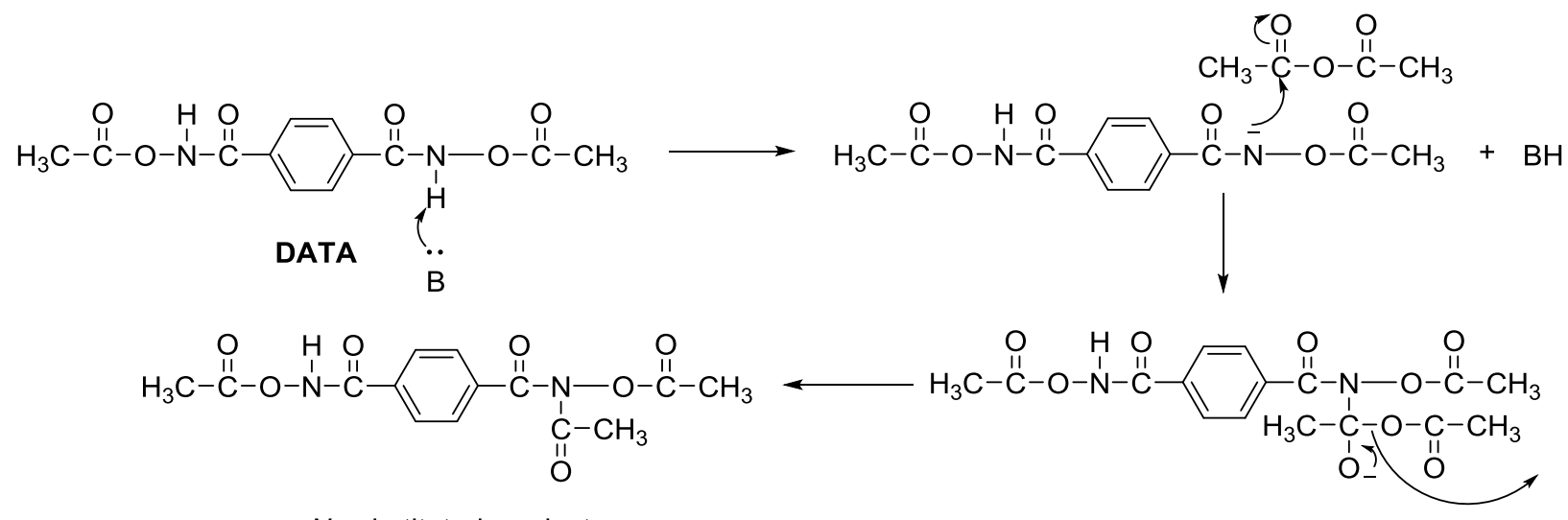

$N$-substituted product

Scheme 2.5 Plausible mechanism for the formation of $N$-substituted DATA

DATA was fully characterized by the IR, ${ }^{1} \mathrm{H}$ NMR, ${ }^{13} \mathrm{C}$ NMR, mass spectroscopy and TGA. The IR spectrum (Figure 2.2) displays the stretching $\mathrm{O}-\left(\mathrm{CH}_{3}\right) \mathbf{C}=\mathbf{O}$ band at $1789 \mathrm{~cm}^{-1}$ and stretching $\mathrm{NH}$ band at $3128 \mathrm{~cm}^{-1}$, which are the characteristic peaks for acetoxy $\mathrm{C}=\mathrm{O}$ and amide $\mathrm{NH}$. There is no peak at $3286 \mathrm{~cm}^{-1}$ and the $\mathrm{NH}$ band separates out, indicating that all the $\mathrm{OH}$ was converted to an ester. The ${ }^{1} \mathrm{H}$ NMR spectrum (Figure 2.3) of DATA shows a new singlet peak at 2.25 ppm, indicating the presence of the $\mathbf{C H}_{3}-\mathrm{C}=\mathrm{O}$ group. There is no peak at $9.14 \mathrm{ppm}$, indicating all the $\mathrm{OH}$ reacted. The $\mathrm{NH}$ peak shifted downfield to $12.52 \mathrm{ppm}$ from $11.37 \mathrm{ppm}$, due 
to the electron withdrawing nature of the acetoxy group. The ratio of aromatic protons to the $\mathrm{CH}_{3}$ protons in DATA are exactly 4:6, further indicating no nucleophilic substitution on the amide. The ${ }^{13} \mathrm{C}$ NMR spectrum (Figure 2.4) of DATA shows two peaks at $170.0 \mathrm{ppm}$ and $165.12 \mathrm{ppm}$, for the $\mathrm{O}-\left(\mathrm{CH}_{3}\right) \mathbf{C}=\mathbf{O}$ and $\mathrm{HN}-\mathbf{C}=\mathbf{O}$, respectively. The methyl carbon appears at $19 \mathrm{ppm}$. The mass spectrum is given in Appendix A (Figure S2.5).

Thermogravimetric analysis (TGA) measures the weight changes of a sample as a function of temperature. The TGA trace of DATA reveals the onset temperature for $1 \%$ weight loss at $150.3{ }^{\circ} \mathrm{C}$ (Figure 2.5), which implies that the Lossen rearrangement of DATA takes place at this specific temperature to generate PPDI in situ. Therefore, it can be proposed that prepolymer/polyurethane be synthesized in one pot from DATA and polyol.

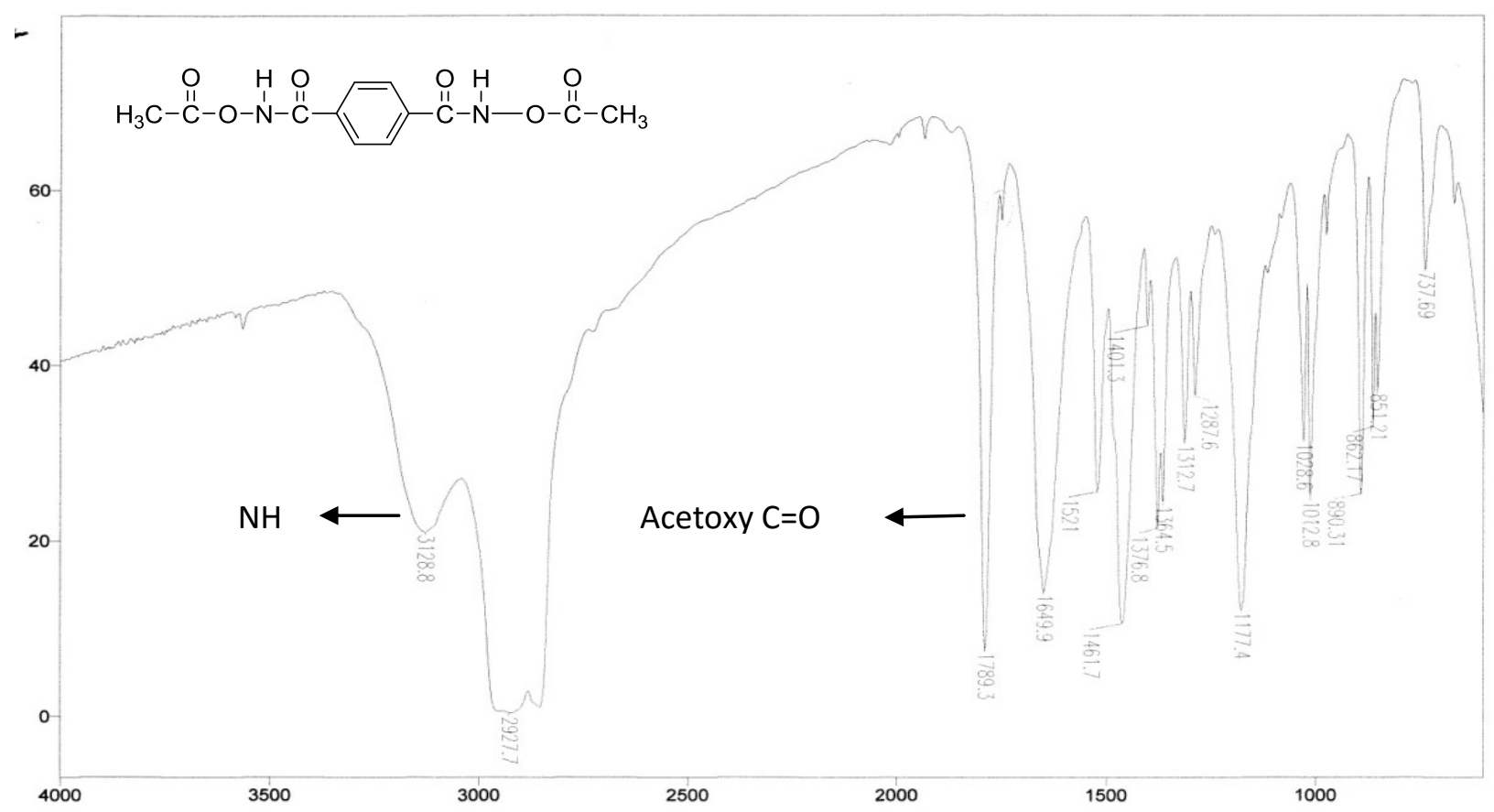

Figure 2.2 IR spectrum of DATA 


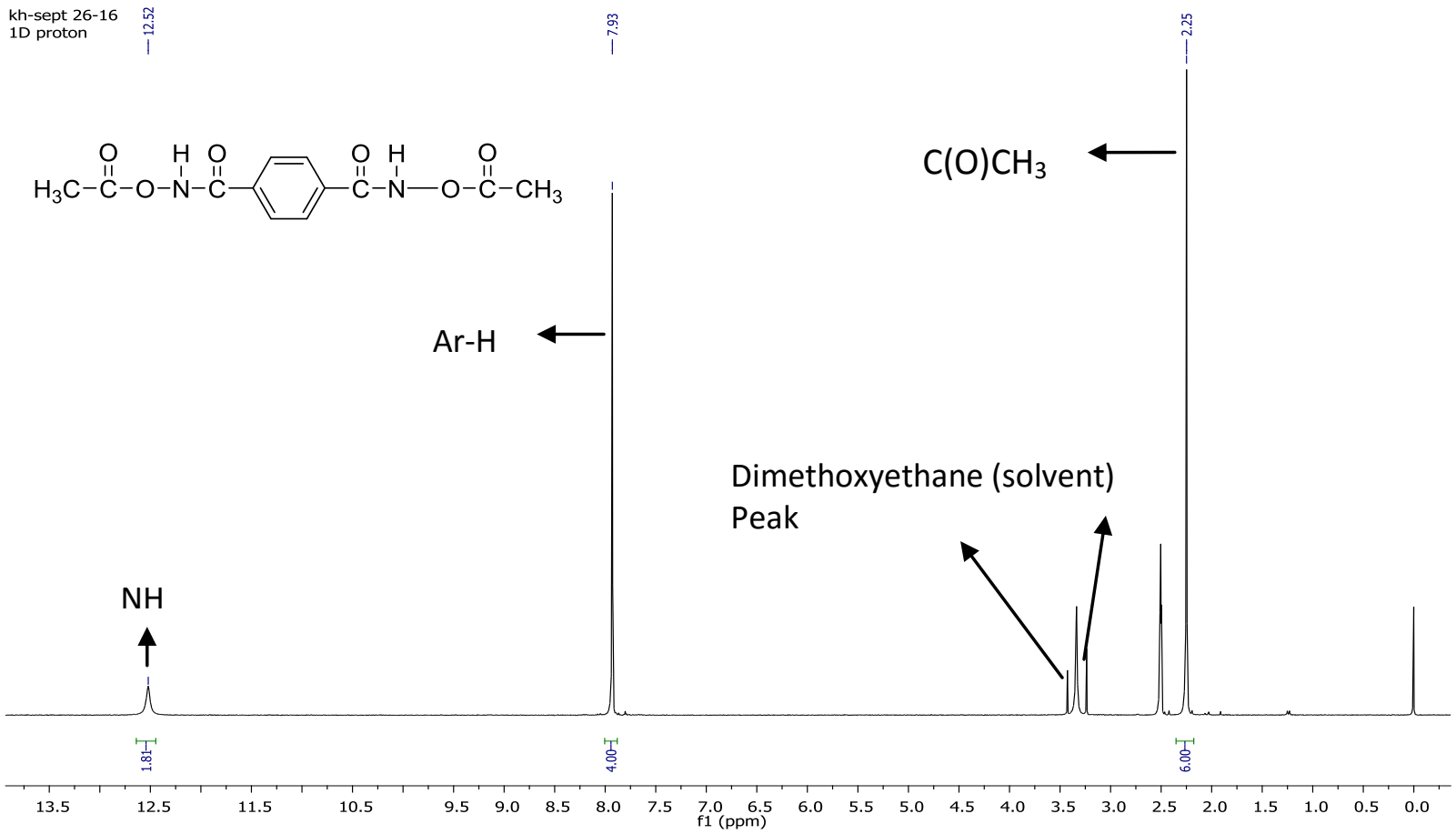

Figure $2.3{ }^{1} \mathrm{H}$ NMR spectrum $\left(300 \mathrm{MHz}, \mathrm{DMSO}-\mathrm{d}_{6}\right)$ of DATA

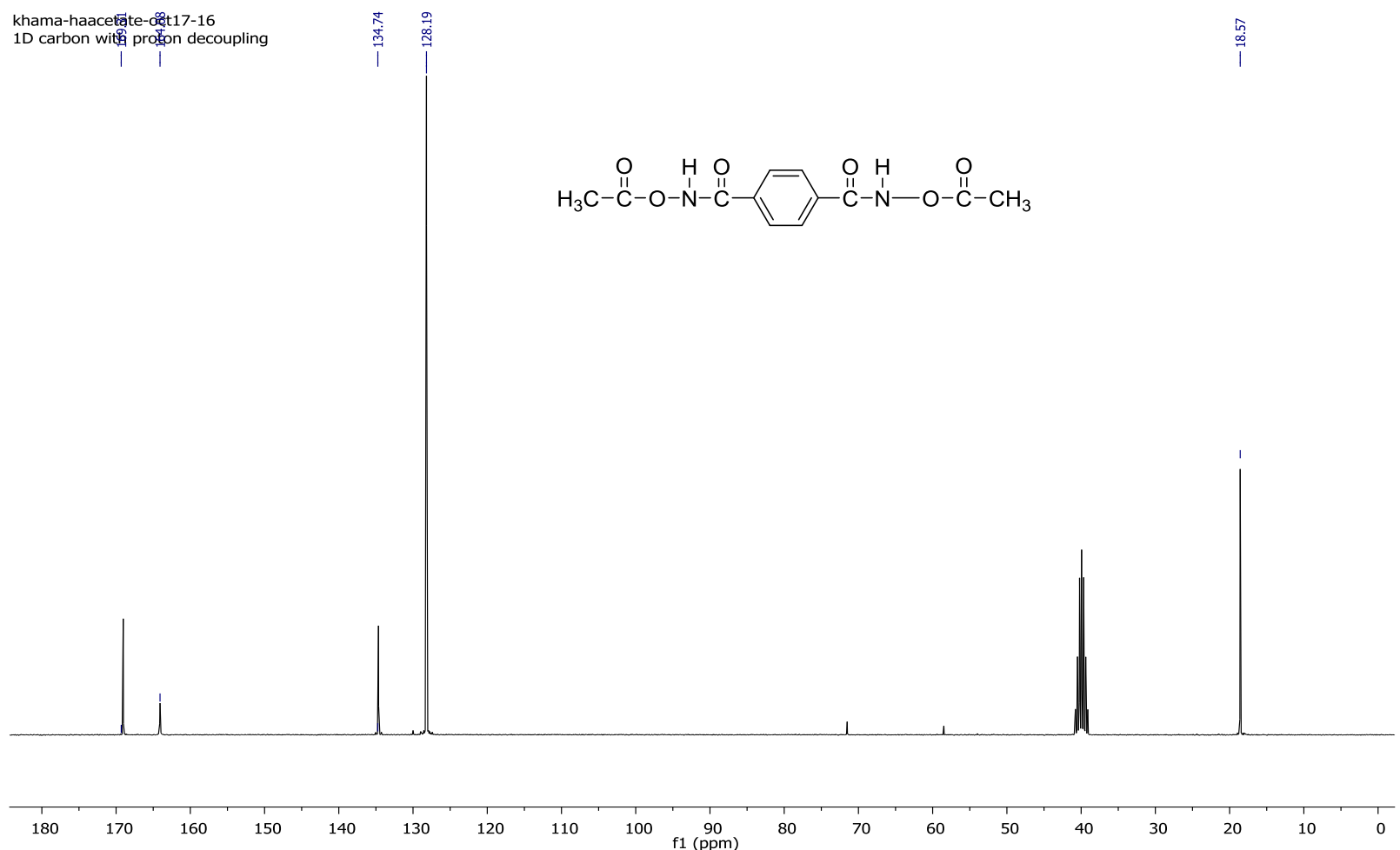

Figure $2.4{ }^{13} \mathrm{C}$ NMR spectrum $\left(75 \mathrm{MHz}\right.$, DMSO- $\left.\mathrm{d}_{6}\right)$ of DATA 


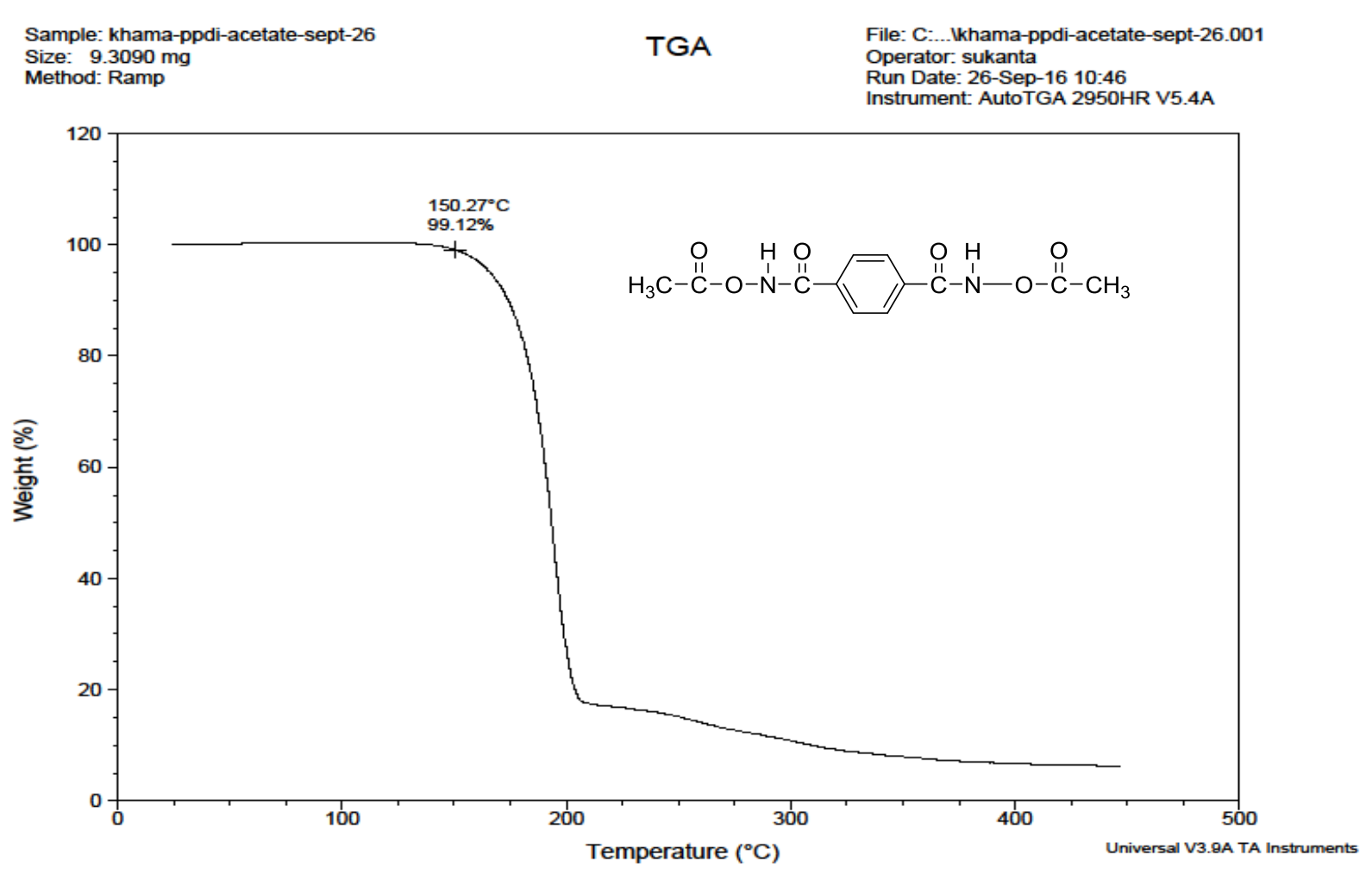

Figure $2.5 \mathrm{TGA}$ trace of DATA $\left(10^{\circ} \mathrm{C} / \mathrm{min}\right.$ in nitrogen $)$

\subsection{Model reactions}

Before making the target prepolymers, we carried out the model reactions of DATA and heptadecane-9-ol, in order to confirm our hypothesis. In addition, characterizations of the model products will help confirm the structures of the target prepolymers. As mentioned earlier, two different reaction conditions are applied herein: (1) without a catalyst at high temperature and (2) with a catalyst at low temperature. Heptadecane-9-ol was chosen because it is expected to impart the solubility to the product for easy isolation and characterization. 


\subsubsection{Reaction of $N^{1}, N^{4}$-diacetoxyterephthalamide and heptadecane-9-ol in absence of a base catalyst}

DATA was heated at $150{ }^{\circ} \mathrm{C}$ in dioctyl terephthalate (DOT) for 9 minutes (Scheme 2.6). The IR spectrum (Figure 2.6) shows the appearance of NCO peak at $2263 \mathrm{~cm}^{-1}$ along with another peak at $2238 \mathrm{~cm}^{-1}$. The later peak has a higher intensity compared to the NCO peak. At that point, the reaction temperature was lowered to $90{ }^{\circ} \mathrm{C}$ and 2 equivalents of 9-heptadecan-9-ol were added. The IR spectrum (Figure 2.7) was taken again after addition of heptadecan-9-ol and showed that an unknown peak at $2338 \mathrm{~cm}^{-1}$ remained and the NCO peak disappeared. The reaction continued for another 1.5 hours and an unknown peak at $2338 \mathrm{~cm}^{-1}$ became very weak. At that point, the products were separated and identified as the urethane compound $\mathbf{1}$ along with some unreacted DATA. The yield of compound 1 was low $(40 \%)$ as expected, due to the low conversion of DATA during the reaction. The important outcome from this model reaction is that at $150{ }^{\circ} \mathrm{C}$ DATA converts to PPDI via Lossen rearrangement as we expected (Scheme 2.7) and then the in situ resulting isocyanate reacts with alcohol to form a urethane compound in two steps in a one-pot reaction.

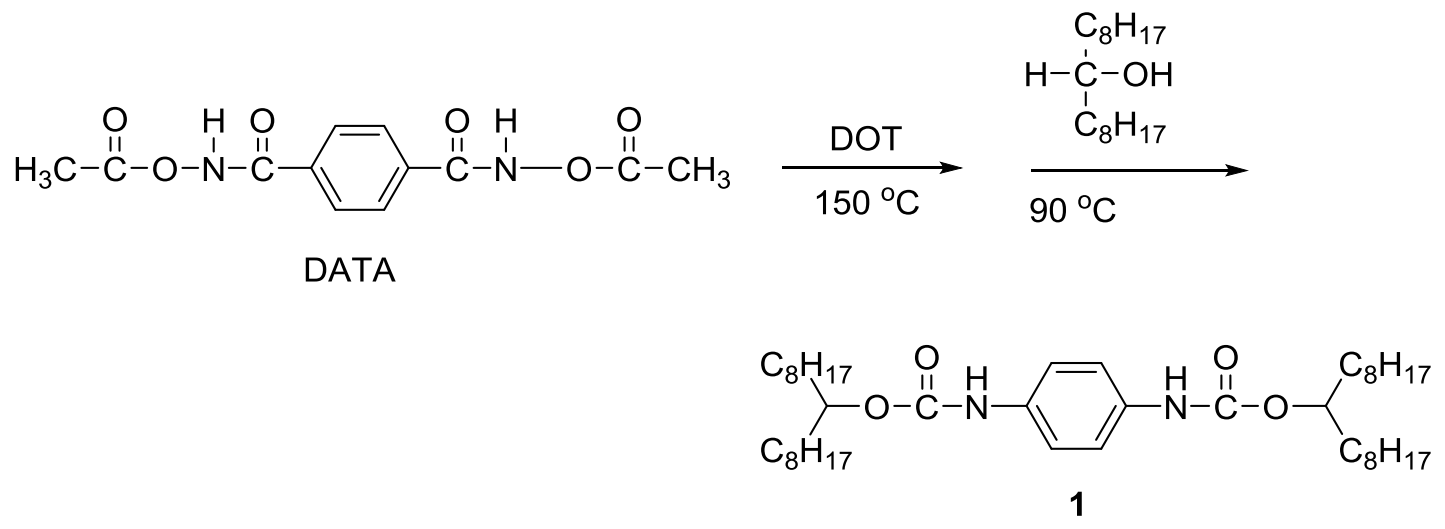

Scheme 2.6 Reaction of DATA and heptadecane-9-ol 
We believe that DATA forms PPDI in situ via Lossen rearrangement (Scheme 2.7), where elimination of the acetate ion is followed by the migration of carbon to nitrogen to yield the isocyanate. ${ }^{16}$ Heptadecane-9-ol as a nucleophile reacts with the in situ generated PPDI to afford compound $\mathbf{1}$.

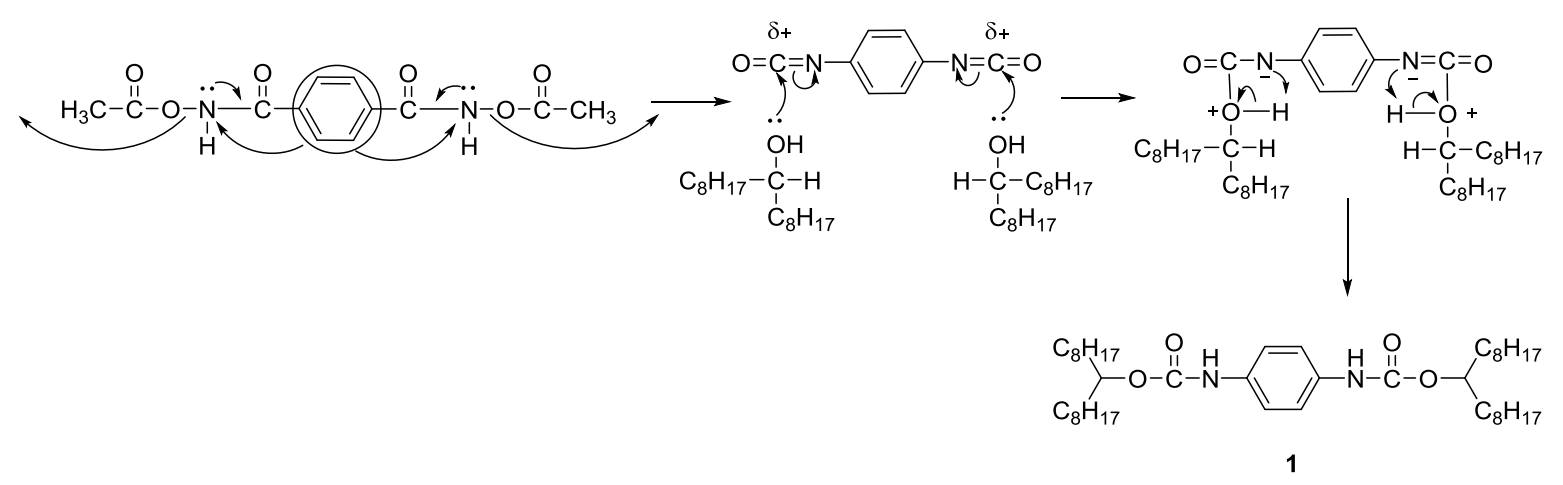

Scheme 2.7 Mechanism for the reaction of DATA and heptadecane-9-ol

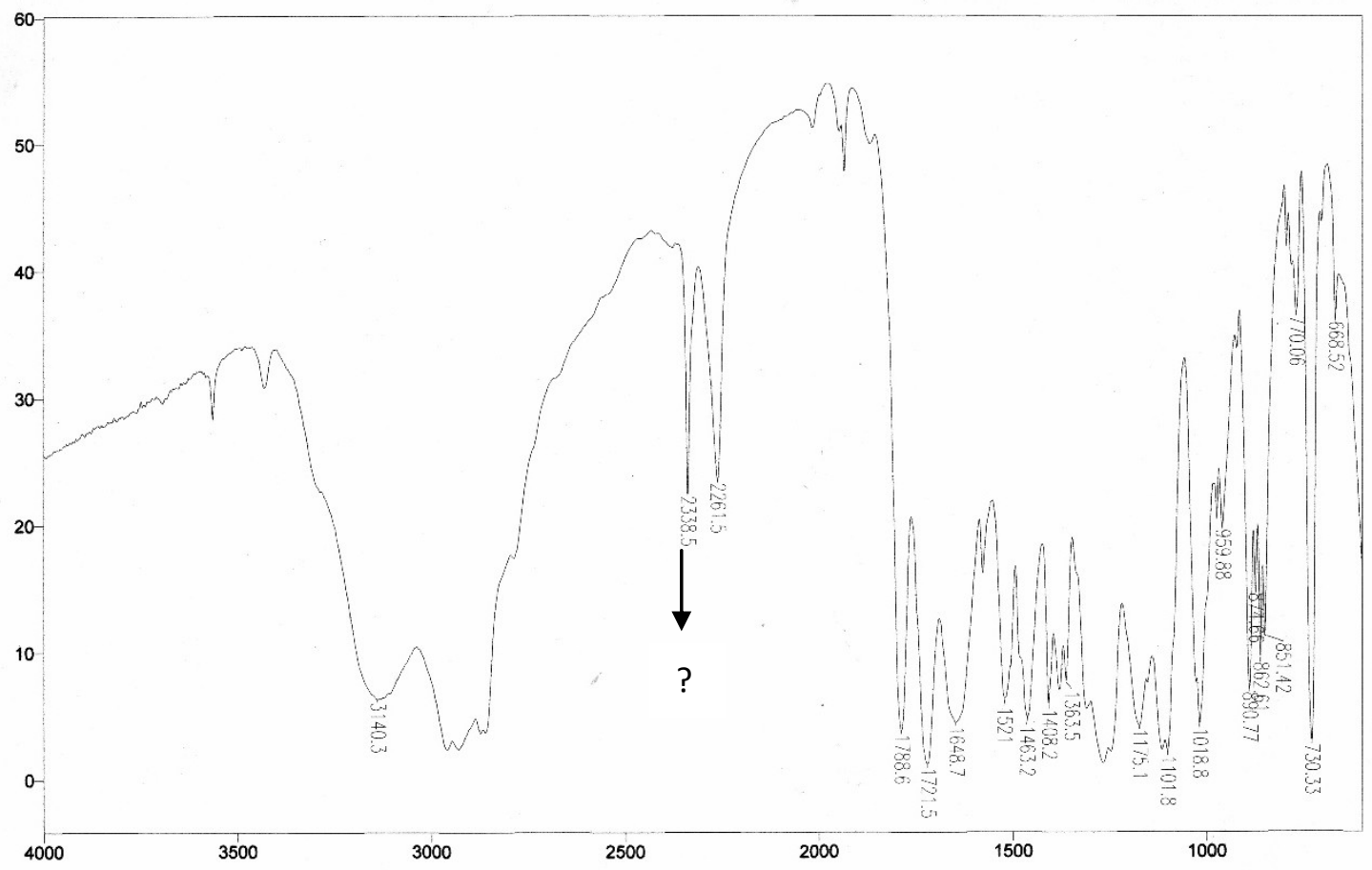

Figure 2.6 IR spectrum of DATA in dioctyl terephthalate at $150{ }^{\circ} \mathrm{C}$ after 9 minutes 


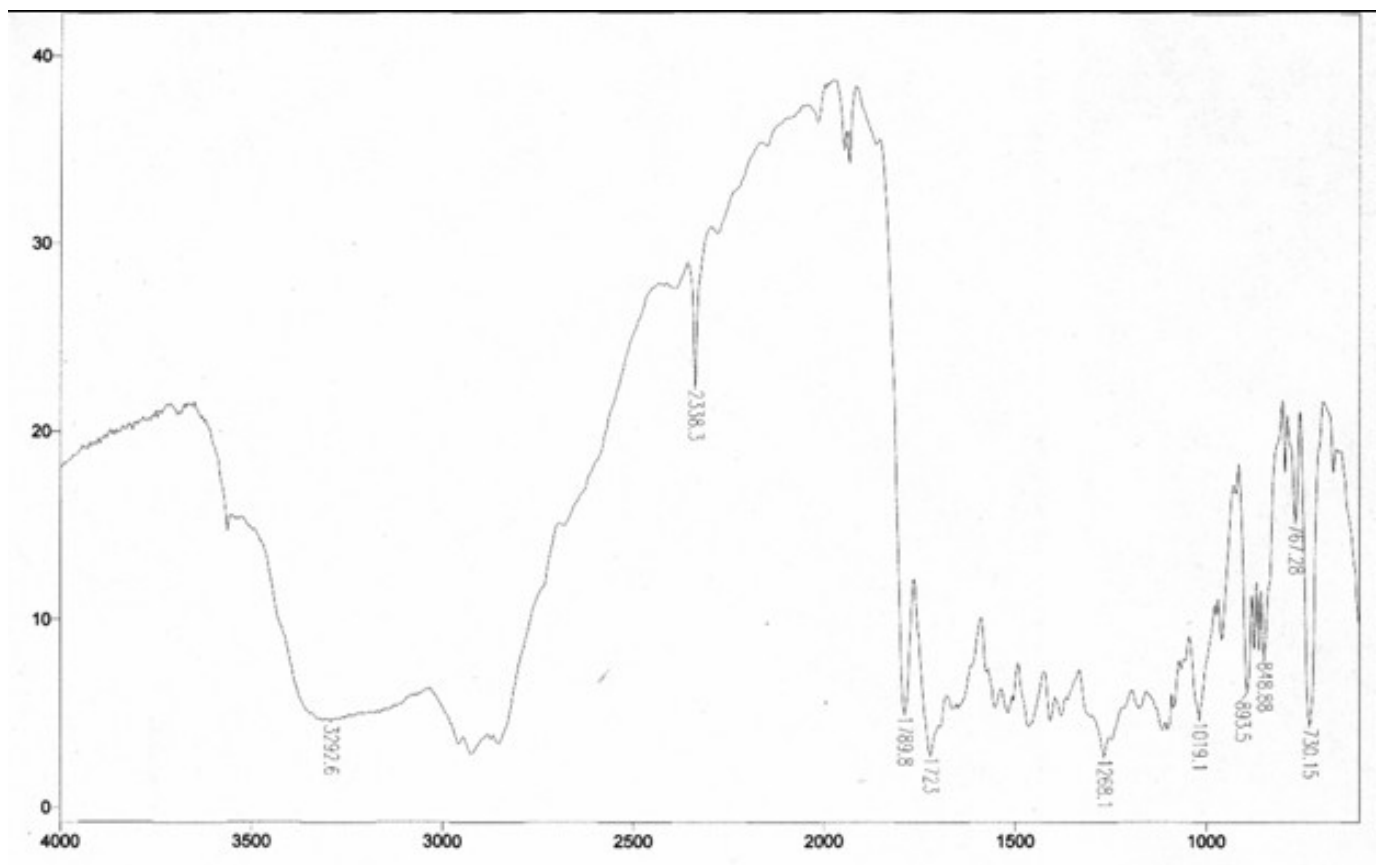

Figure 2.7 IR spectrum of the reaction mixture after adding 9-heptadecanol as shown in Scheme 2.6

\subsubsection{Reaction of $N^{1}, N^{4}$-diacetoxyterephthalamide and heptadecane-9-ol in the presence of} a base catalyst

DATA was heated with heptadecane-9-ol in the presence of TBD as a base catalyst at 80 ${ }^{\circ} \mathrm{C}$ according to Scheme 2.8. After the reaction, we got the same result as described above, compound 1 and unreacted DATA. At the same time, we studied the reaction with another base, 1,8-diazabicyclo[5.4.0]undec-7-ene (DBU). The $\mathrm{p} k_{\mathrm{a}}$ values of DBU and TBD are 13.5 and 15.2, respectively, and thus should be basic enough to remove the amide proton from DATA to promote the Lossen rearrangement. Accordingly, the base deprotonates the $\mathrm{NH}$ of the amide part of DATA and then elimination of acetate ion occurs, followed by the migration of carbon to nitrogen to form the isocyanate (Scheme 2.9). The isolated yields of compound $\mathbf{1}$ from the 
reactions using either TBD or DBU are similar, in 50-52\%. All the spectra are given in Appendix A (Figures S2.6-2.12).

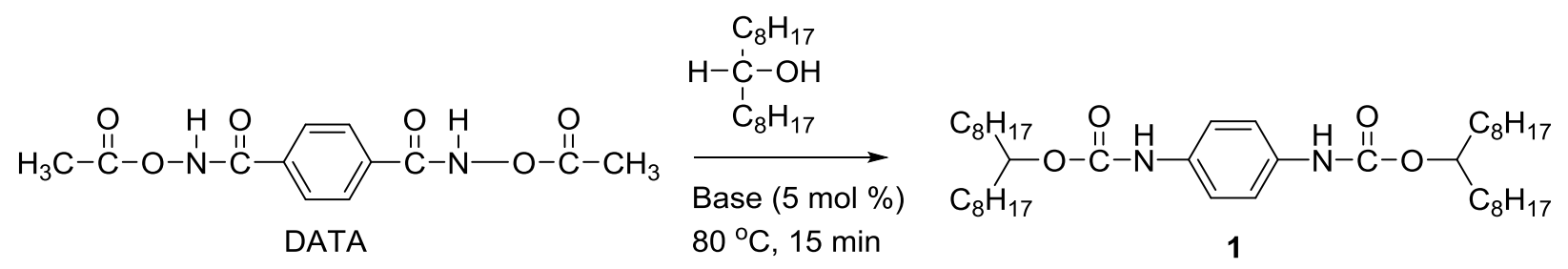

Scheme 2.8 Reaction of DATA and heptadecane-9-ol in the presence of a base catalyst
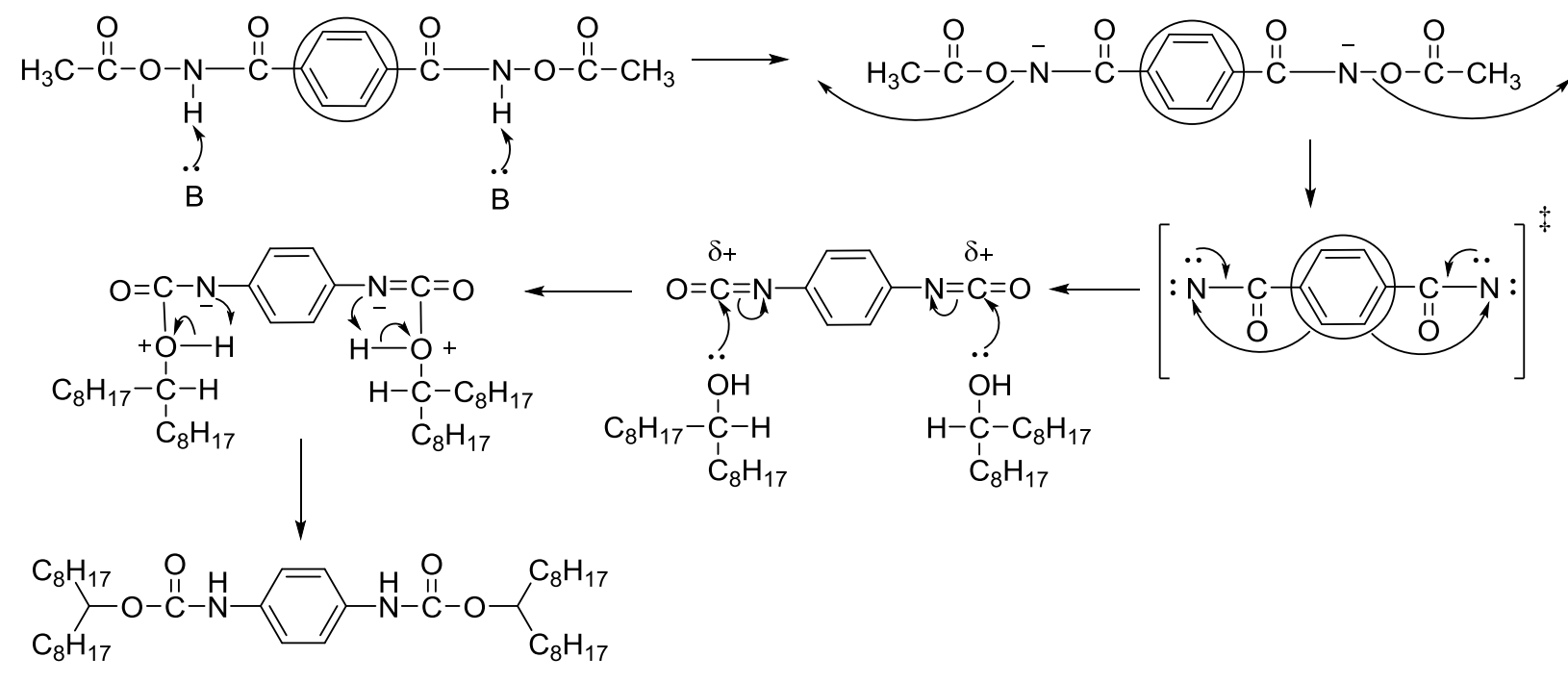

Scheme 2.9 Reaction mechanism of DATA and heptadecane-9-ol in the presence of a base

It was thought at that time that the unknown peak at $2338 \mathrm{~cm}^{-1}$ is an intermediate, which is gradually converted to PPDI in situ. To figure out what this intermediate might be, when the peak at $2338 \mathrm{~cm}^{-1}$ appeared in a high intensity ethanol was added in order to trap the intermediate compound. However, there was no compound that could relate to the peak at 2338 $\mathrm{cm}^{-1}$ in IR spectrum. A short investigation was done in order to identify the source of the mysterious IR peak at $2338 \mathrm{~cm}^{-1}$. As we know, the asymmetric peak for $\mathrm{CO}_{2}$ in the gas phase is at $2345 \mathrm{~cm}^{-1}$, being close to $2338 \mathrm{~cm}^{-1}$. In a test experiment, $\mathrm{CO}_{2}$ gas was bubbled into DOT at 
room temperature and the IR spectra of the $\mathrm{CO}_{2}$-containing DOT were taken at 5 minute intervals for 15 minutes (Figures S13-S14). DOT does not have any peak at $2338 \mathrm{~cm}^{-1}$ in the IR spectrum. However, the IR spectrum (Figure 2.8) shows a peak at $2336 \mathrm{~cm}^{-1}$ for the $\mathrm{CO}_{2}$ containing DOT, with a very similar shape to the one for the unknown found in the model reactions. The peak intensity increased with the concentration of $\mathrm{CO}_{2}$ in DOT as well. The test simply confirms that the peak at $2338 \mathrm{~cm}^{-1}$ observed during the model reactions is due to $\mathrm{CO}_{2}$ trapped or dissolved in the mixture. During the reaction, $\mathrm{CO}_{2}$ could be formed from the reaction as a result of hydrolysis of isocyanate or from the air as we did not degas the reaction mixture.

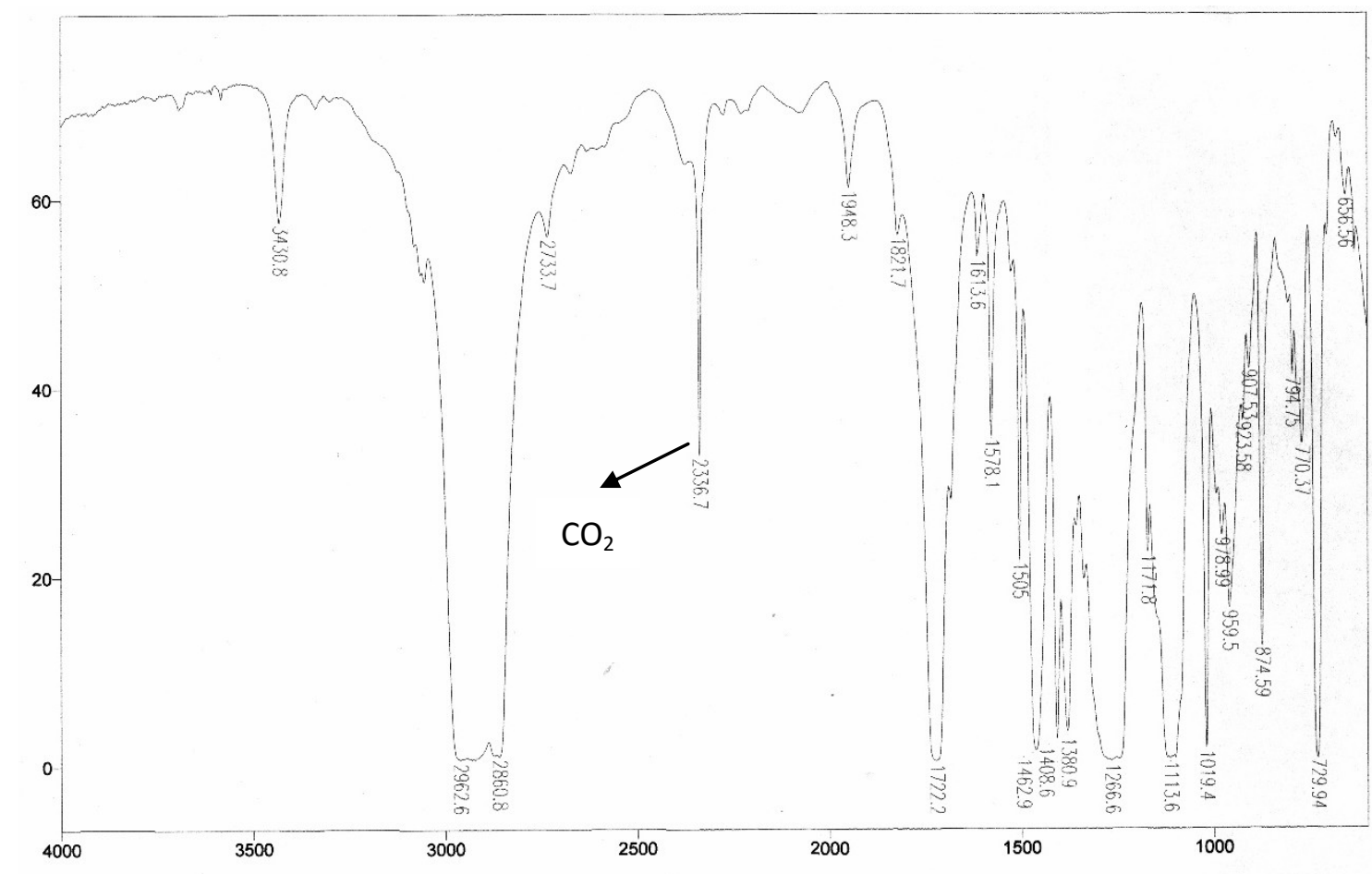

Figure 2.8 IR spectrum of dioctyl terephthalate after bubbling in $\mathrm{CO}_{2}$ at room temperature for 15 minutes

\subsubsection{Reactions of $\boldsymbol{N}$-acetoxybenzamide and $\boldsymbol{n}$-butanol with and without a base catalyst}

Before moving to the target prepolymers, another model reaction was carried out using $N$-acetoxybenzamide (ABA) and $n$-butanol (Scheme 2.10). We intended to do the same reaction 
with $\mathrm{ABA}$, following the same reaction conditions for model reactions in sections 2.4 .1 and 2.4.2. The characterizations and spectra of ABA are in Appendix A ((Figures S2.15-2.18). TGA of ABA (Figure S2.19) indicates the onset temperature for Lossen rearrangement of the acetoxyamide group at $100{ }^{\circ} \mathrm{C}$, much lower than that of DATA. ABA was synthesized (Scheme 2.10) by acylation of $N$-hydroxybenzamide with acetic anhydride. ${ }^{17-19}$ The synthesis of ABA without the $N$-acylation can easily be controlled by the amount of base. ${ }^{20,21}$

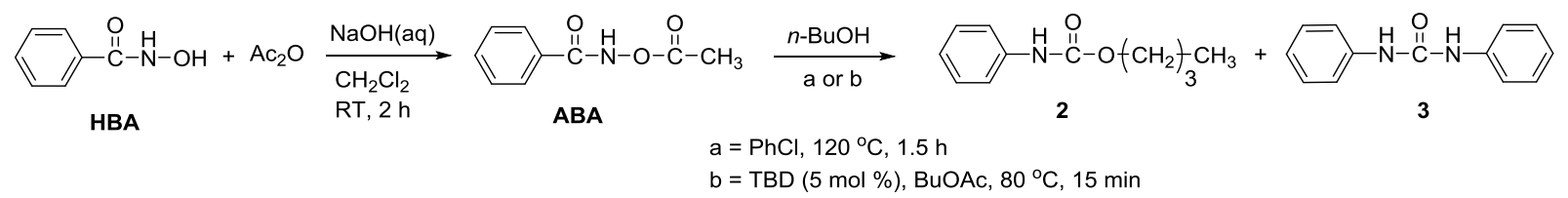

Scheme 2.10 Reactions of ABA with $n$-butanol under two different reaction conditions

After the reaction with $n$-butanol, the products were separated and characterized by spectroscopic analysis and elemental analysis (Figures S2.20-S2.27). It was found that the major product was urethane compound 2 (35\%), along with the urea $3(25 \%)$ and the unreacted ABA. The characterizations of compound $\mathbf{3}$ are consistent with the known data in the literature. ${ }^{22,23}$ No urea product was found from the reaction of DATA, which implies a different reactivity between DATA and ABA.

From the studies of these model reactions, we have learned that the Lossen rearrangement of DATA can take place under the two different conditions: at $150{ }^{\circ} \mathrm{C}$ without a base and at 80 ${ }^{\circ} \mathrm{C}$ with a base. The investigation revealed that in the presence of TBD catalyst the desired Lossen rearrangement can be accomplished in a very short time and at a lower temperature since the in situ generated isocyanate was not detectable by routine sampling for IR analysis. We have further learned that we can make the target prepolymers terminated either with the acetoxyamide (AA) group (as a latent $\mathrm{NCO}$ ) or with the $\mathrm{NCO}$ group. There are several variables like 
temperature, time and the amount of catalyst need to be further optimized for the prepolymer synthesis.

\subsection{Synthesis of NCO-terminated prepolymer from DATA and PTMEG}

The NCO-terminated prepolymer P1 was synthesized from DATA and PTMEG at high temperature without a base catalyst (Scheme 2.11). PTMEG stands for poly(tetramethylene ether glycol) with a molecular weight 1000. The reaction was carried out using 3:1 molar ratio of DATA and PTMEG at $150{ }^{\circ} \mathrm{C}$ and the reaction progress was monitored by IR spectroscopy (Figure 2.9). IR spectra were taken at different time intervals and observed the formation of isocyanate with the peak at $2268 \mathrm{~cm}^{-1}$, since it is known that the NCO stretching appears at 2261-

$2265 \mathrm{~cm}^{-1} \cdot{ }^{24}$ It was also observed that the urethane $\mathrm{C}=\mathrm{O}$ stretching peak at around $1730 \mathrm{~cm}^{-1}$ increased in intensity with simultaneous decrease of the intensity of acetate $\mathrm{C}=\mathrm{O}$ peak at 1788 $\mathrm{cm}^{-1}$ (Figure 2.9a). After 90 minutes, the peaks at $3304 \mathrm{~cm}^{-1}$ and $2268 \mathrm{~cm}^{-1}$ appeared (Figure $2.9 \mathrm{~b}$ ), indicating the presence of the urethane bond and isocyanate. It was also noticed that the peak for acetate $\mathrm{C}=\mathrm{O}$ was very weak and the peak for urethane $\mathrm{C}=\mathrm{O}$ stretching was very intense. If the reaction mixture was heated for more than 90 minutes, then the peak intensity of NCO decreased (Figure S2.28), presumably due to the formation of side products. In general, the formation of urethane bond (-NH-COO-) is evident by the peaks around $3300-3320 \mathrm{~cm}^{-1}$, the stretching vibration of $\mathrm{OCN}$ and $\mathrm{NH}$ bending around $1526 \mathrm{~cm}^{-1}$ and $\mathrm{C}-\mathrm{O}-\mathrm{C}$ stretching around $1057-1103 \mathrm{~cm}^{-1}$. More importantly, the sharp peaks around $1696-1730 \mathrm{~cm}^{-1}$ and $1222 \mathrm{~cm}^{-1}$ are typical for the stretching vibration of $\mathrm{C}=\mathrm{O}$ and asymmetric stretching vibration of $\mathrm{C}-\mathrm{O}$ of the urethane bond, respectively. $^{25}$ 


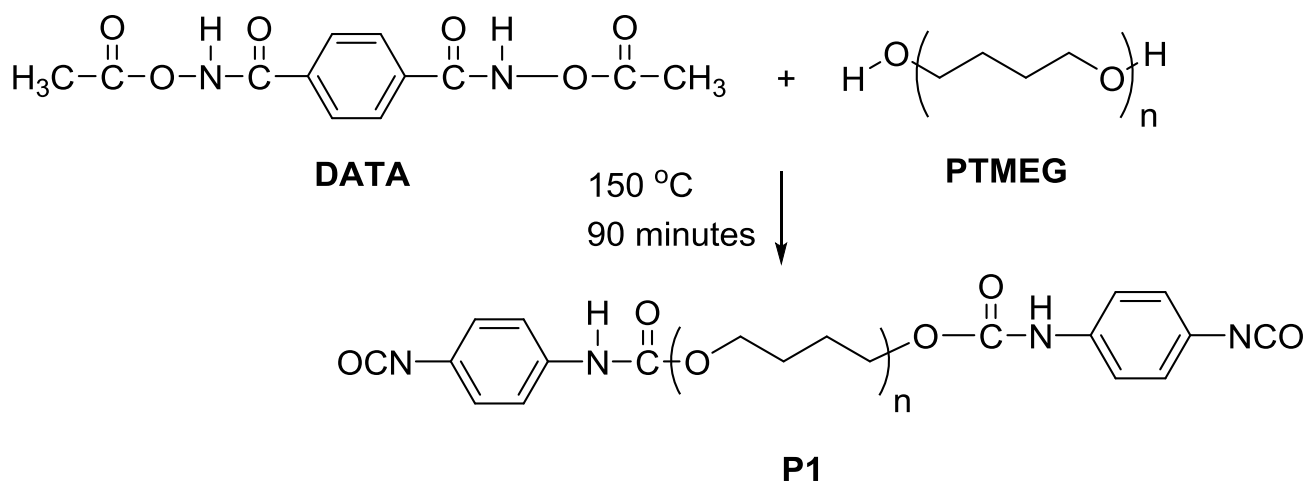

Scheme 2.11 Synthesis of prepolymer P1

After performing several experiments, it can be concluded that the reaction time of 90 minutes is optimal for the synthesis of P1. Acetic acid formed during the reaction should be removed in order to prevent the reaction with isocyanate. In our reaction, a higher molar ratio of DATA was used in order to facilitate the in situ formation of PPDI and NCO-terminated prepolymer. 


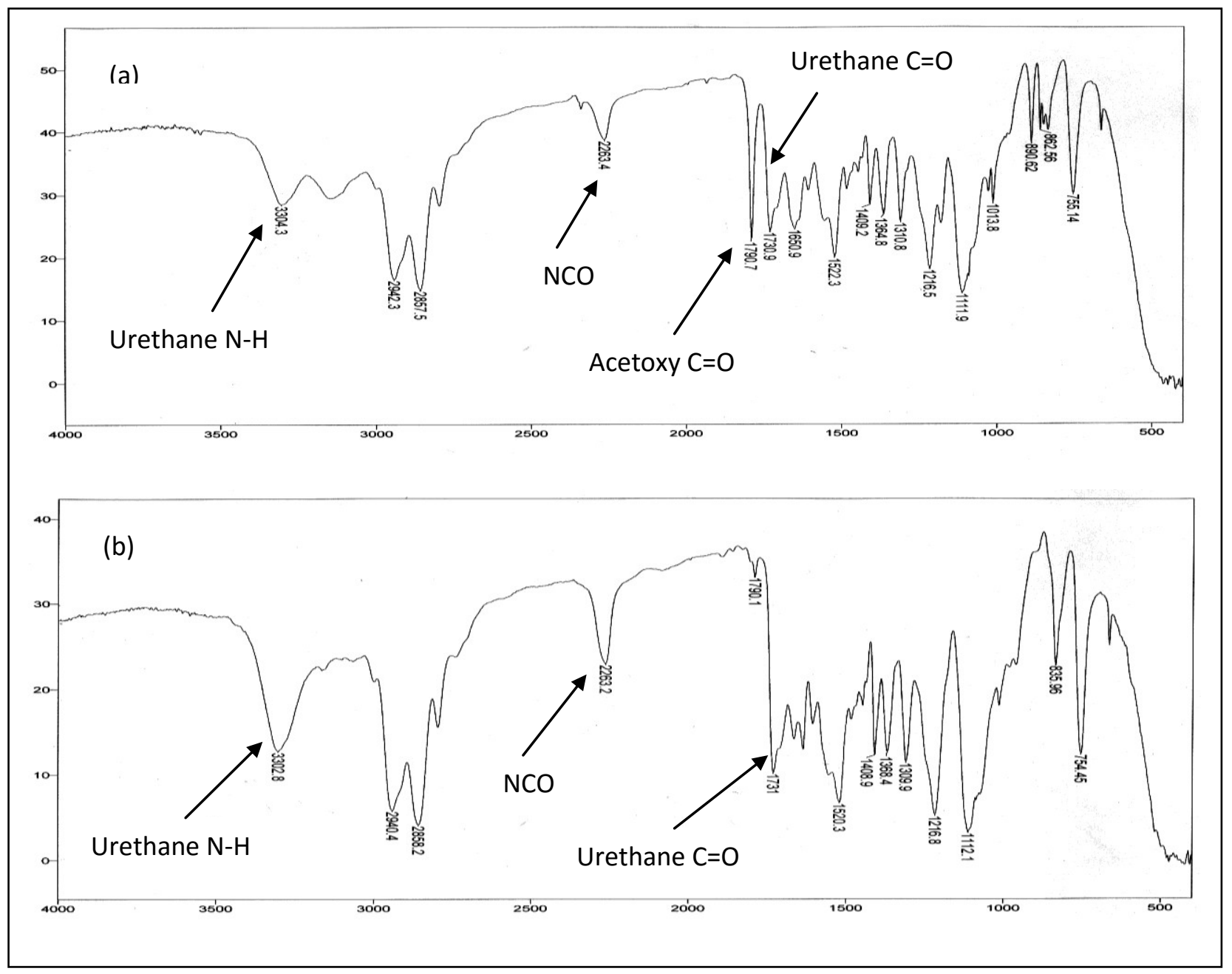

Figure 2.9 IR spectra of the reaction mixtures (DATA and PTMEG) taken at different time intervals, (a) 60 minutes, and (b) 90 minutes

To confirm the formation of prepolymer P1 from DATA and PTMEG, the same polymer was synthesized from PPDI and PTMEG (Scheme 2.12). PPDI and PTMEG were combined in a 3:1 molar ratio and the reaction mixture was heated at $150{ }^{\circ} \mathrm{C}$ for 5 to 15 minutes while monitoring by IR spectroscopy. The IR spectrum (Figure 2.10) displays the peaks at 3296, 2262 and $1730 \mathrm{~cm}^{-1}$, which are exactly the same as those for prepolymer P1 made from DATA and 
PTMEG (Figure 2.9). The fingerprint regions of both the IR spectra have the same profile, which further confirms the successful synthesis of prepolymer P1 from DATA.

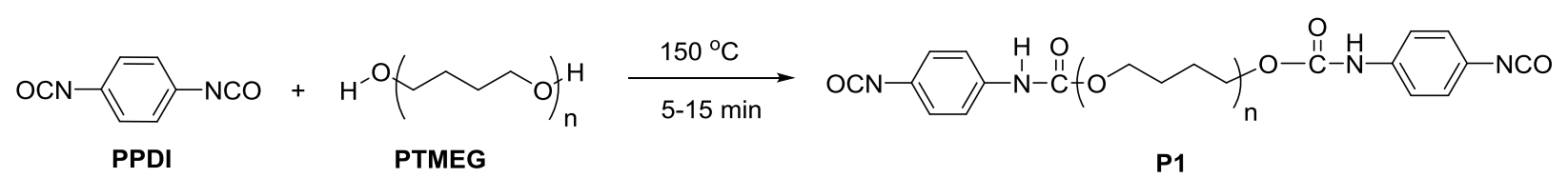

Scheme 2.12 Synthesis of NCO-terminated prepolymer with PPDI

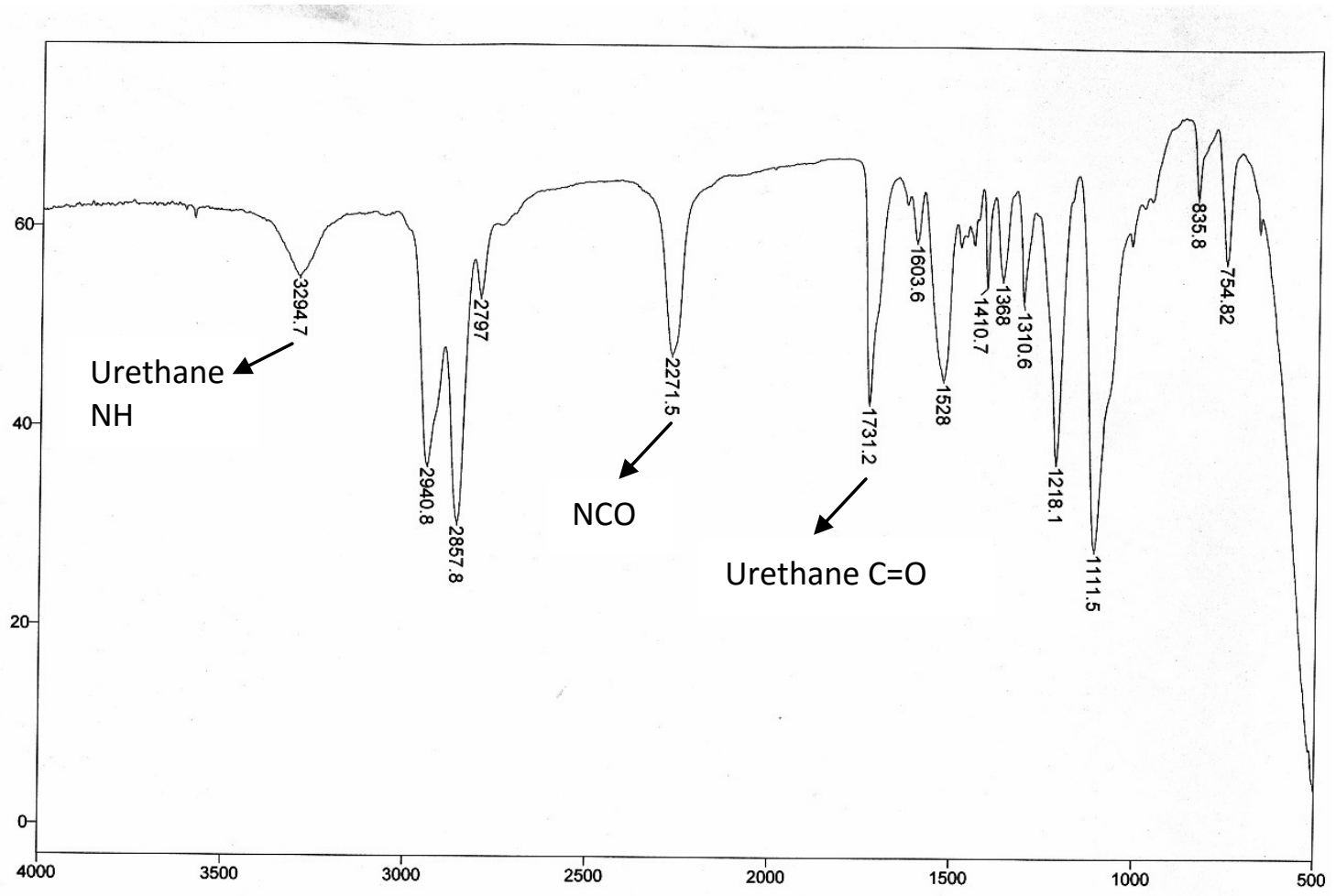

Figure 2.10 IR spectrum of reaction mixture of PPDI and PTMEG

\subsection{One-pot synthesis of polyurethane from DATA and PTMEG}

Our research on the chemistry of DATA continued by optimizing the reaction time and temperature, and by exploring the use of TBD as a catalyst. Accordingly, the direct synthesis of polyurethane (P2) in one pot form DATA and PTMEG was explored. Initially, the reaction was 
carried out using a 1:1 molar ratio of DATA and PTMEG at $130{ }^{\circ} \mathrm{C}$ in the presence of $5 \mathrm{~mol} \%$ TBD (Scheme 2.13). After 2 minutes of the reaction, the IR spectrum displays characteristic peaks at 3304 and $1727 \mathrm{~cm}^{-1}$ for $\mathrm{NH}$ and $\mathrm{C}=\mathrm{O}$ of urethane (Figure 2.11). There were no peaks at $1790 \mathrm{~cm}^{-1}$ and $3144 \mathrm{~cm}^{-1}$ from DATA, indicating a complete conversion of DATA by Lossen rearrangement and subsequent reaction with PTMEG to form a PU within 2 minutes. In the presence of TBD catalyst, the desired Lossen rearrangement can be accomplished in a very short time and at a lower temperature. Even without observing the peak at $2263 \mathrm{~cm}^{-1}$ for the NCO group, the reaction certainly proceeds via in situ formation of isocyanate. This study shows that by using a base catalyst and adjusting the ratio of reactants, the polyurethane can be synthesized from a non-isocyanate monomer, such as DATA.

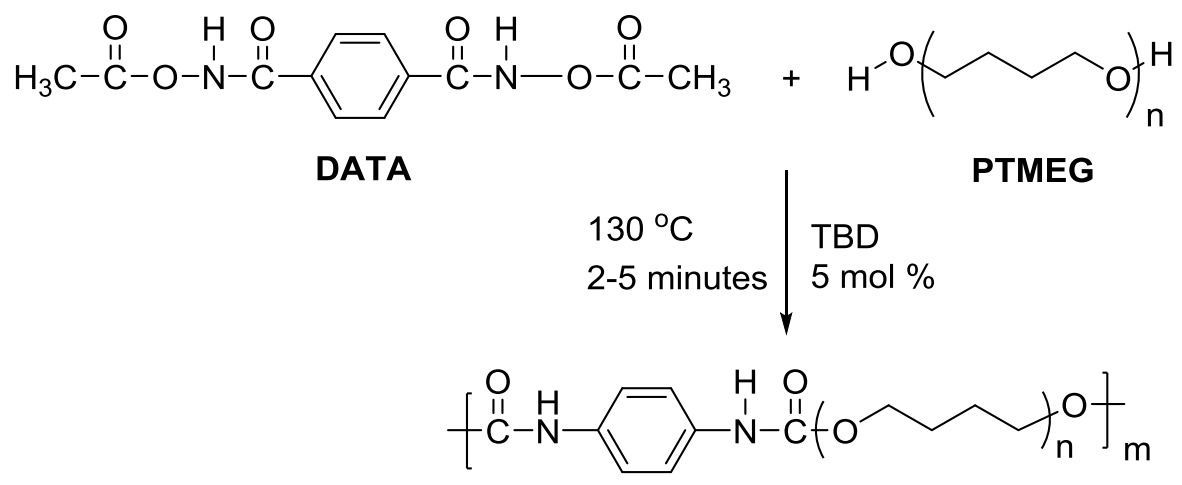

P2

Scheme 2.13 One-pot synthesis of polyurethane from DATA and PTMEG in the presence of a base catalyst 


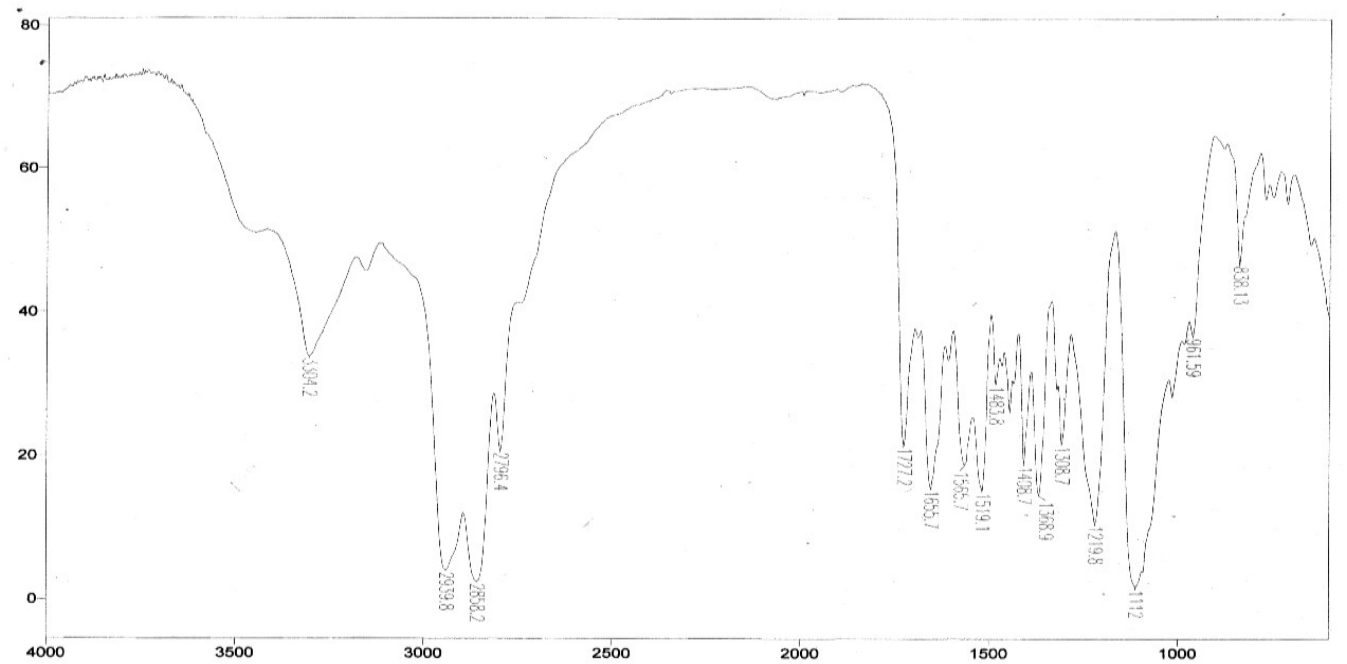

Figure 2.11 IR spectrum of the reaction mixture of DATA and PTMEG (1:1 molar ratio) at 130 ${ }^{\circ} \mathrm{C}$ in the presence of $5 \mathrm{~mol} \% \mathrm{TBD}$ after 2 minutes

\subsection{Synthesis of acetoxyamide-terminated prepolymer from DATA and PTMEG}

Based on the results obtained from our previous experiments, by running the same reaction in the presence of $5 \mathrm{~mol} \% \mathrm{TBD}$ but at a lower temperature, one should be able to selectively make the acetoxyamide-terminated prepolymers. Thus, under an argon atmosphere, a mixture of DATA and PTMEG (2.2:1 molar ratio) in the presence of $5 \mathrm{~mol} \% \mathrm{TBD}$ was heated at $80{ }^{\circ} \mathrm{C}$ (Scheme 2.14) for 15 minutes. The IR spectra were acquired every 5 minutes. Figures 2.12(a) and (b) show the IR spectra of the reaction mixture after 5 and 15 minutes. In Figure 2.12(a), there is no peak at $2263 \mathrm{~cm}^{-1}$ for $\mathrm{NCO}$ stretching. It also displays a strong urethane $\mathrm{C}=\mathrm{O}$ peak at $1732 \mathrm{~cm}^{-1}$ and an acetate $\mathrm{C}=\mathrm{O}$ peak at $1789 \mathrm{~cm}^{-1}$, along with a weak peak $2338 \mathrm{~cm}^{-1}$. In Figure 2.12(b), there is still no peak at around $2263 \mathrm{~cm}^{-1}$ for the NCO group. The urethane $\mathrm{C}=\mathrm{O}$ peak at $1731 \mathrm{~cm}^{-1}$ became more intense and the acetoxy $\mathrm{C}=\mathrm{O}$ peak at $1789 \mathrm{~cm}^{-1}$ became weaker. From these IR spectra, it can be concluded that under these conditions prepolymer $\mathbf{P 3}$ is produced and the end group is AA rather than NCO group. 

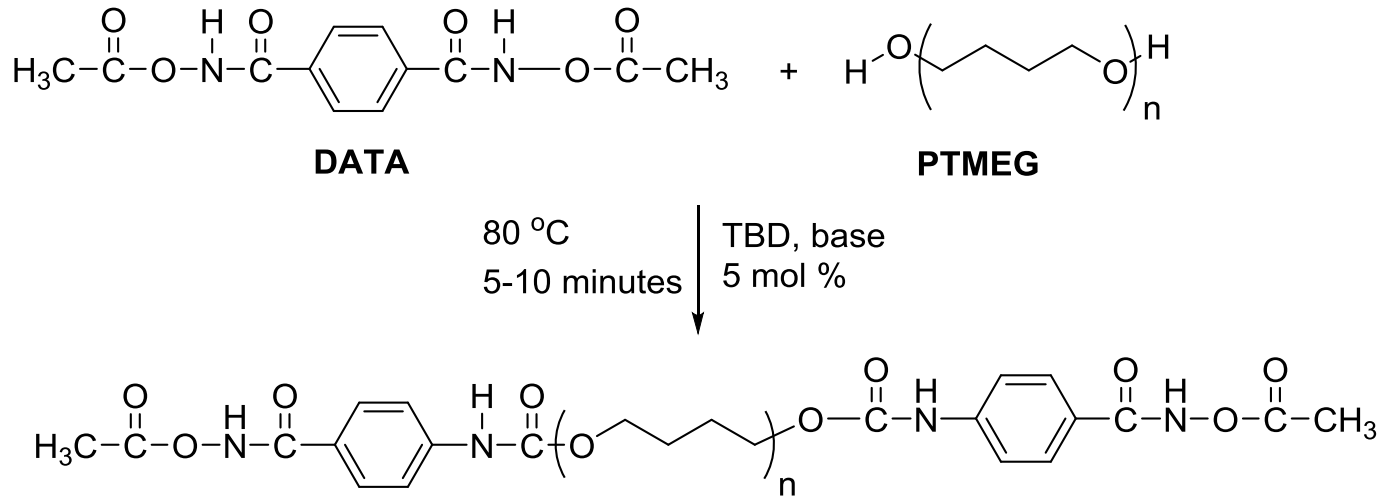

P3

Scheme 2.14 Synthesis of AA-terminated prepolymer P3 in the presence of a base catalyst

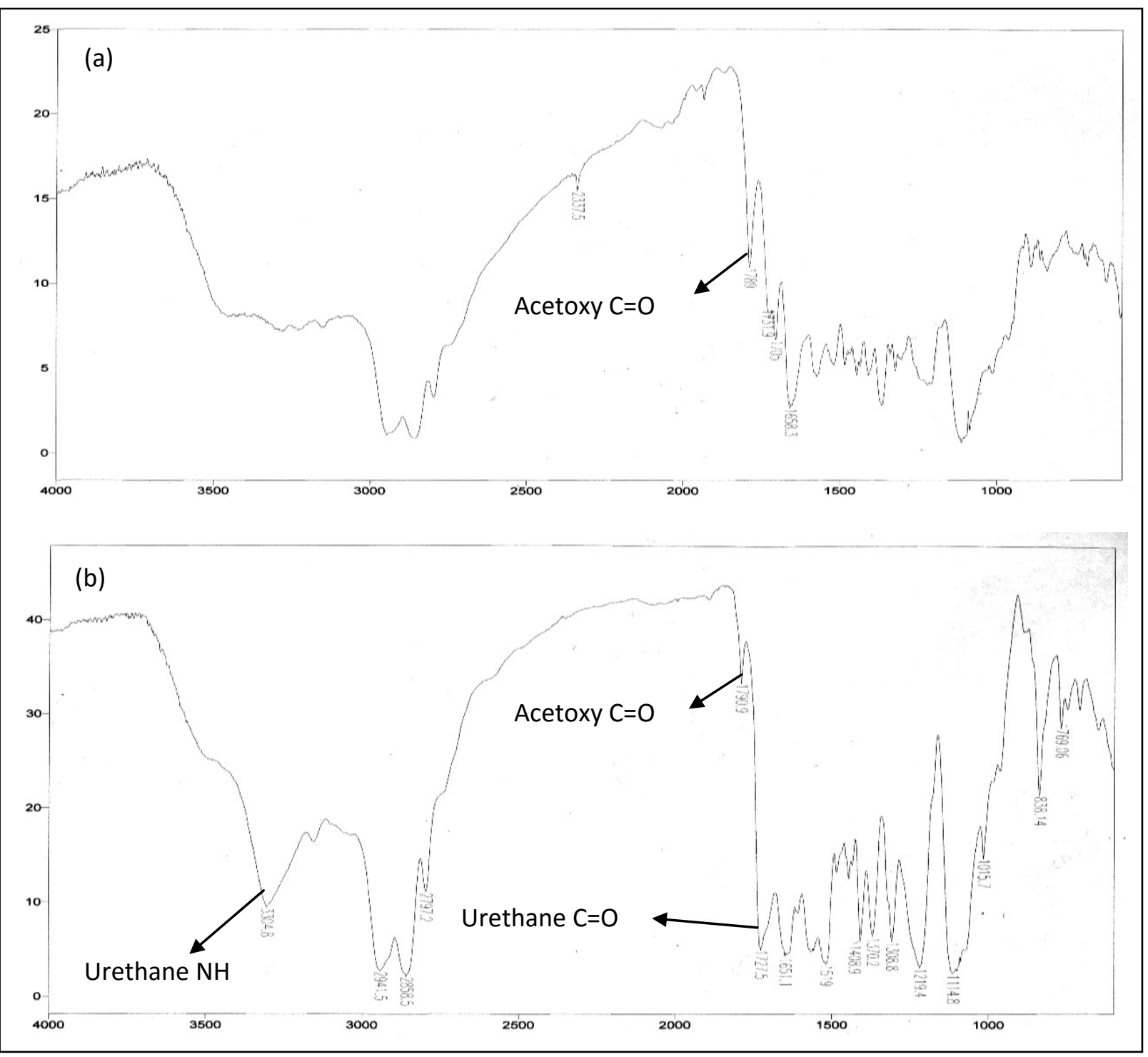


Figure 2.12 The IR spectra of reaction mixture of DATA and PTMEG (2.2:1 molar ratio) heated at $80{ }^{\circ} \mathrm{C}$ in the presence of $5 \mathrm{~mol} \% \mathrm{TBD}$ after (a) 5 minutes (b) 15 minutes

\subsection{One-pot synthesis of polyurethane from DATA}

Prepolymer P3 is terminated with AA and should be able to convert to prepolymer P1 by simple heating at elevated temperatures. Thus, when prepolymer $\mathbf{P 3}$ was heated from $115^{\circ} \mathrm{C}$ to $130{ }^{\circ} \mathrm{C}$, no peak was observed at $2263 \mathrm{~cm}^{-1}$ in the IR spectrum, indicating no conversion to isocyanate. When the temperature increased to $150{ }^{\circ} \mathrm{C}$ and after 5 to 10 minutes, the IR spectrum displayed a peak at $2152 \mathrm{~cm}^{-1}$ for the NCO group (Figure 2.13). The peak at $1790 \mathrm{~cm}^{-1}$ for the AA group disappeared. This result implies that prepolymer $\mathbf{P 3}$ was successfully converted to prepolymer $\mathbf{P 1}$.<smiles>CC(=O)ONC(=O)c1ccc(NC(=O)OCCCCC(C)(C)OC(=O)Nc2ccc(C(=O)NOC(C)=O)cc2)cc1</smiles>

P3

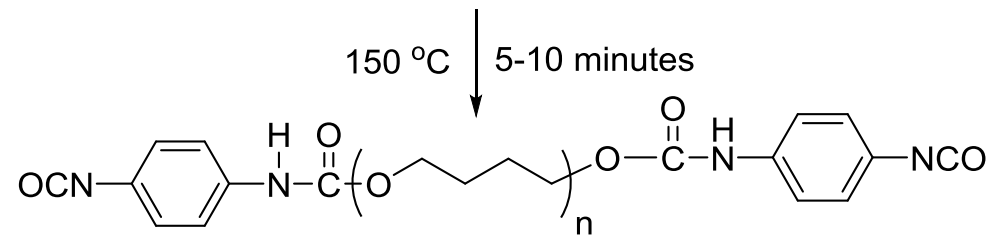

P1
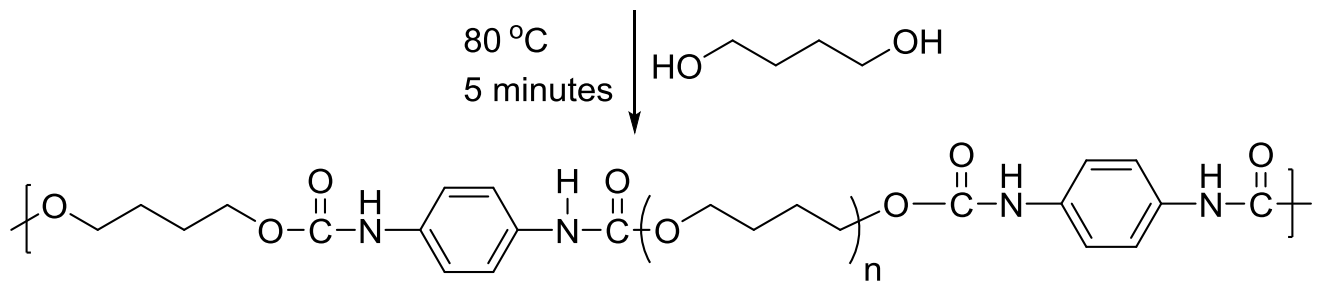

P4

Scheme 2.15 In situ conversion of acetoxyamide-terminated prepolymer P3 to NCO-terminated prepolymer P1 and subsequent chain extension with BDO to form polyurethane $\mathbf{P 4}$ 


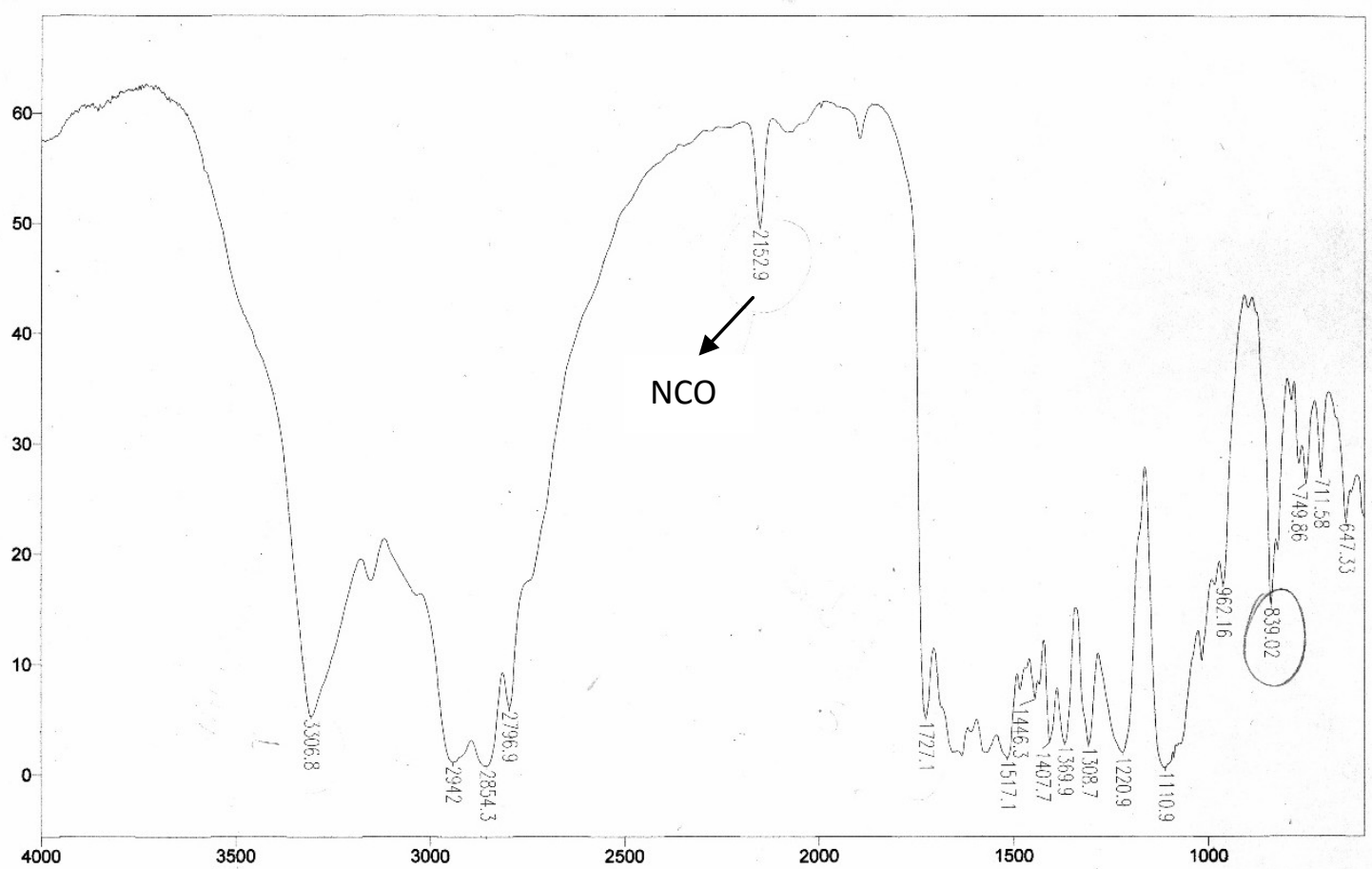

Figure 2.13 IR spectrum for the in situ conversion of prepolymers P3 to P1

To further confirm the formation of reactive prepolymer P1, a molar equivalent of 1,4butanediol (BDO) was added to the mixture of DATA and PTMEG after being heated for $10 \mathrm{~min}$ (Scheme 2.15). After addition and heating at $80{ }^{\circ} \mathrm{C}$ for $5 \mathrm{~min}$, the IR spectrum (Figure 2.14) shows the intense $\mathrm{C}=\mathrm{O}$ band at $1729 \mathrm{~cm}^{-1}$ and $\mathrm{NH}$ band at $3314 \mathrm{~cm}^{-1}$. Therefore, polyurethane $\mathbf{P 4}$ is formed and it is elastic. 


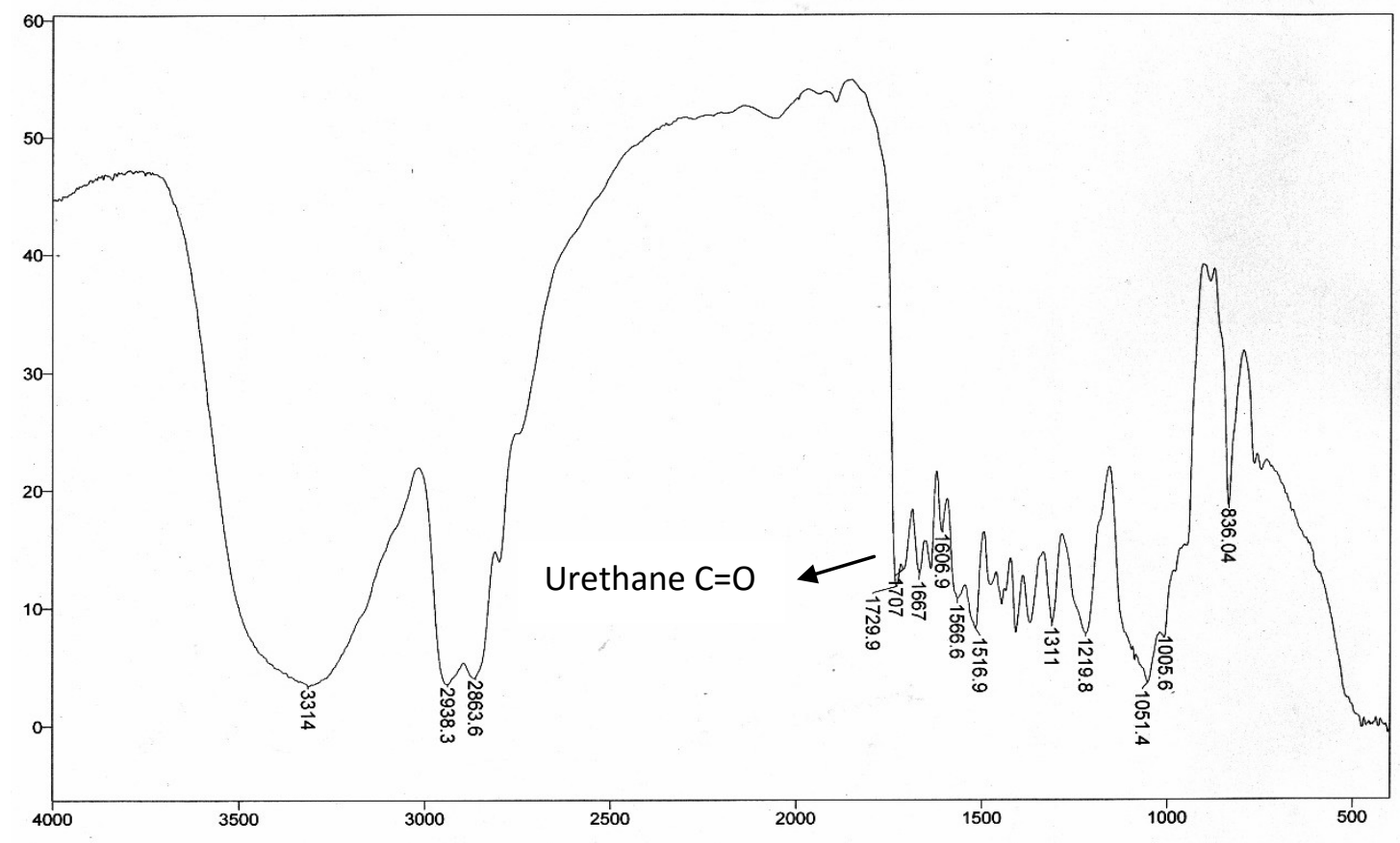

Figure 2.14 IR spectrum of the reaction mixture after addition of BDO in 5 minutes at $80{ }^{\circ} \mathrm{C}$ to the product from the reaction of DATA and PTMEG

The investigation into the effect of reaction conditions on the Lossen reaction of DATA have drawn some conclusive findings so far: (1) By heating at $150{ }^{\circ} \mathrm{C}$, it is feasible to obtain the NCO-terminated prepolymer (e.g., P1) from DATA and a polyol (e.g., PTMEG), (2) In the presence of base (e.g., TBD) at $80{ }^{\circ} \mathrm{C}$, it is feasible to synthesize the acetoxyamide-terminated or so-called blocked prepolymer (e.g., P3), (3) It is feasible to further convert the acetoxyamideterminated prepolymer to NCO-terminated prepolymer by heating at $150{ }^{\circ} \mathrm{C}$ (e.g., from $\mathbf{P 3}$ to P1), (4) It is feasible to directly synthesize a polyurethane from DATA and a polyol (e.g., P2). For industrial applications, AA-terminated prepolymers (e.g., P3) can be stored over a long period of time and can be formulated with alcohol curative as one-component polyurethane precursors or with amine as polyurea precursors. 


\subsection{Conclusion}

By changing the reaction conditions, NCO-terminated and acetoxyamide-terminated prepolymers were synthesized via Lossen rearrangement. By heating at $150{ }^{\circ} \mathrm{C}$, the reaction of an excess of $N^{1}, N^{4}$-diacetoxyterephthalamide and PTMEG afforded the NCO-terminated prepolymer. Addition of 1,4-butanediol to the resultant prepolymer produced a polyurethane. In the presence of a base at $80^{\circ} \mathrm{C}$, an acetoxyamide-terminated prepolymer was selectively formed. This prepolymer could be converted to the NCO-terminated prepolymer upon heating at $150{ }^{\circ} \mathrm{C}$. The acetoxyamide-terminated prepolymers are considered the latent or blocked NCO-terminated prepolymers. Accordingly, $N^{1}, N^{4}$-diacetoxyterephthalamide is considered to be the equivalent of PPDI suitable for the syntheses of polyurethane or polyurea.

\subsection{Experimental Section}

\section{Materials}

All reagents were purchased from commercial sources and were used as received unless otherwise noted. PTMEG (Terathane 1000) was dried in a vacuum oven at $70{ }^{\circ} \mathrm{C}$ for $5 \mathrm{~h}$ before use. 1,4-Butanediol was dried in a vacuum oven at $50{ }^{\circ} \mathrm{C}$ for $5 \mathrm{~h}$ before using. DOT (boiling point of $400{ }^{\circ} \mathrm{C}$ ) was purchased from Sigma-Aldrich and was purified by distillation to remove low boiling $\left(\sim 100^{\circ} \mathrm{C}\right)$ impurities.

\section{General methods}

Thin layer chromatography (TLC) was used to monitor reaction progress and to check the purity of the synthesized products. The material used for TLC was silica gel, $60 \mathrm{G} / \mathrm{UV}_{254}$ (Macherey-Nagel). Column chromatography was used for the separation of pure product from a reaction mixture. The crude product was often purified by column chromatography. The 
chromatography column was prepared using slurry silica gel (Silicycle Chemical Division, 70$230 \mathrm{mesh}$ ) as the stationary phase.

Fisher-John's melting point apparatus was used for recording the melting points of all the synthesized compounds by thin disc method. The melting points were not corrected. ${ }^{1} \mathrm{H}$ and ${ }^{13} \mathrm{C}$ NMR spectra were recorded on a Bruker Avance Digital $300 \mathrm{MHz}\left(300\right.$ and $75 \mathrm{MHz}$ for ${ }^{1} \mathrm{H}$ and ${ }^{13} \mathrm{C}$ NMR, respectively). Resonances were quoted on the $\delta$ scale relative to tetramethylsilane (TMS, $\delta=0$ ) as an internal standard. For ${ }^{1} \mathrm{H}$ NMR spectra, the following abbreviations have been used: $\mathrm{s}=$ singlet, $\mathrm{d}=$ doublet, $\mathrm{t}=$ triplet, $\mathrm{q}=$ quartet, $\mathrm{m}=$ multiplet. Infrared measurements were performed on a Varian 1000 IR Scimitar series spectrophotometer. The measuring mode was $\% \mathrm{~T}$ (percentage transmittance). The bands were expressed in $\mathrm{cm}^{-1}$ (per centimeter). Mass spectra were measured with a Micromass Quattro LC-ESI (EI). Thermo gravimetric analysis was carried out in nitrogen on a Hi-Res TGA 2950 thermo gravimetric analyzer with a heating rate of $10{ }^{\circ} \mathrm{C} \mathrm{min}^{-1}$. Standard differential scanning calorimetric analysis was carried out under a nitrogen atmosphere on a TA DSC Q100 with a heating rate $10^{\circ} \mathrm{C} \mathrm{min}^{-1}$.

\section{Synthesis}

$N^{1}, N^{4}$-dihydroxyterephthalamide

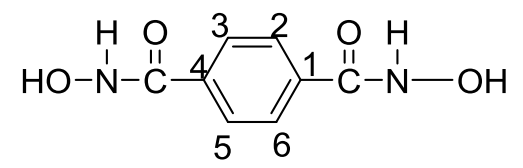

Diethyl terephthalate $(10.0 \mathrm{~g}, 0.0449 \mathrm{~mol})$ was dissolved in $150 \mathrm{~mL}$ of methanol in a 500 mL 3-neck, round-bottomed flask at room temperature. In another 2-neck, round-bottomed flask, $\mathrm{NaOH}(10.8 \mathrm{~g}, 0.271 \mathrm{~mol})$ and $\mathrm{NH}_{2} \mathrm{OH} . \mathrm{HCl}(9.48 \mathrm{~g}, 0.136 \mathrm{~mol})$ were dissolved in $85 \mathrm{~mL}$ of water. The basic hydroxylamine solution was added dropwise through a dropping funnel to the 
terephthalate solution at $0{ }^{\circ} \mathrm{C}$ and the reaction mixture was then stirred at $55^{\circ} \mathrm{C}$ for $48 \mathrm{~h}$. After cooling to room temperature, the reaction mixture was neutralized by $5 \% \mathrm{HCl}(275 \mathrm{~mL})$ and the precipitate was removed by filtration. The solid was stirred in hot methanol for 5 minutes. The solid product was obtained in $57 \%$ yield after drying in a vacuum oven for $6 \mathrm{~h}$ at $50{ }^{\circ} \mathrm{C}$. m.p. $\sim 245^{\circ} \mathrm{C}$ decomposed. ${ }^{12}$ IR (Nujol) $v_{\max } / \mathrm{cm}^{-1} 3286(\mathrm{NH}, \mathrm{OH}), 1642(\mathrm{O}=\mathrm{CNH}), 1602(\mathrm{O}=\mathrm{CNH})$, 1565 (C-N). ${ }^{1} \mathrm{H}$ NMR (300 MHz, DMSO-d $)$ ) $\delta 11.37$ (s, 2NH), 9.14 (s, 2OH), 7.81 (s, 4Ar-H). ${ }^{13} \mathrm{C}$ NMR $\left(75 \mathrm{MHz}, \mathrm{DMSO}-\mathrm{d}_{6}\right) \delta 163.8(\mathrm{C}=\mathrm{O}), 135.49(\mathrm{Ar}-\mathrm{C} 1, \mathrm{C} 4), 127.37(\mathrm{Ar}-\mathrm{C} 2, \mathrm{C} 3, \mathrm{C} 5$, C6). MS (EI): Exact mass calculated for $\mathrm{C}_{8} \mathrm{H}_{8} \mathrm{~N}_{2} \mathrm{O}_{4}[\mathrm{M}]^{+}$: 196.05000 , found: 196.04625 .

$N^{l}, N^{4}$-diacetoxyterephthalamide

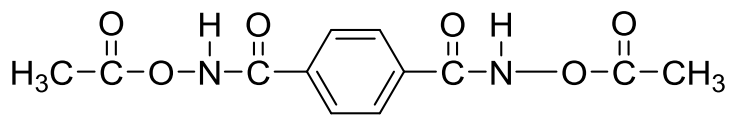

Under an atmosphere of argon in a $100 \mathrm{~mL}$ round-bottom flask, a mixture of $N^{1}, N^{4}$ dihydroxyterephthalamide $(3.70 \mathrm{~g}, 18.8 \mathrm{mmol})$, acetic anhydride $(3.94 \mathrm{~g}, 38.7 \mathrm{mmol})$ and $\mathrm{N}, \mathrm{N}-$ diisopropylethyl amine (4.6 mL, $39.6 \mathrm{mmol})$ in $45 \mathrm{~mL}$ of dry dimethoxymethane was refluxed at $80{ }^{\circ} \mathrm{C}$ for $2 \mathrm{~h}$. The resultant mixture was cooled down and filtered, followed by washing with $\mathrm{CHCl}_{3}$. The product was dried in a vacuum oven for 3 hours at around $50{ }^{\circ} \mathrm{C}$ and obtained as a white solid (4.72 g, $90 \%$ yield). m.p. $\sim 150{ }^{\circ} \mathrm{C}$ decomposed. IR (Nujol) $v_{\max } / \mathrm{cm}^{-1} 3128(\mathrm{~N}-\mathrm{H}$ stretching), $1789\left(\mathrm{O}=\mathrm{C}\right.$ stretching for acetoxy), $1649\left(\mathrm{O}=\mathrm{C}\right.$ stretching for amide), $1521(\mathrm{C}-\mathrm{N}) .{ }^{1} \mathrm{H}$ NMR (300 MHz, DMSO-d 6 ) $\delta 12.52(\mathrm{~s}, 2 \mathrm{NH}), 7.93(\mathrm{~s}, 4 \mathrm{Ar}-\mathrm{H}), 2.25\left(\mathrm{~s}, 3 \mathrm{CH}_{3}-\mathrm{H}\right) .{ }^{13} \mathrm{C}$ NMR $(75$ MHz, DMSO-d $\left.{ }_{6}\right) \delta 170.0(\mathrm{C}=\mathrm{O}), 165.12(\mathrm{C}(\mathrm{O}) \mathrm{O}), 134.74(\mathrm{Ar}-\mathrm{C} 1$ and $\mathrm{C} 4), 128.19(\mathrm{Ar}-\mathrm{C} 2, \mathrm{C} 3$, $\mathrm{C} 5$ and $\mathrm{C} 6), 18.57\left(\mathrm{CH}_{3}-\mathrm{C}\right)$. MS (ESI, DMSO/Acetonitrile): Exact mass calculated for $\mathrm{C}_{12} \mathrm{H}_{12} \mathrm{~N}_{2} \mathrm{O}_{6}[\mathrm{M}+\mathrm{Na}]^{+}: 303.0700$, found: 303.0593 . 
NCO-terminated prepolymer P1<smiles>CC(=O)OCCCCC(C)(C)OC(=O)Nc1ccc([N+](=O)[O-])cc1</smiles>

A flame-dried $25 \mathrm{~mL}$ two-neck round-bottomed flask was kept under argon for 15 min and then $0.128 \mathrm{~g}(0.458 \mathrm{mmol})$ of DATA and $0.153 \mathrm{~g}(0.153 \mathrm{mmol})$ of PTMEG were added to the flask that was placed in a pre-heated oil bath at $150{ }^{\circ} \mathrm{C}$. The progress of the reaction was monitored by IR spectroscopy. After 90 min, prepolymer P1 was obtained as amber and sticky liquid. IR ( $\mathrm{NaCl}$ plate) $v_{\max } / \mathrm{cm}^{-1} 3312.8$ (N-H stretching), 2263.2 (NCO asymmetric stretching), $1731 \& 1707$ (urethane, $\mathrm{C}=\mathrm{O}$ stretching), 1520.3 (N-H bending and $\mathrm{C}-\mathrm{N}$ stretching), 1216.8 (CO stretching), 1112.1 (C-O-C stretching).

Polyurethane P2

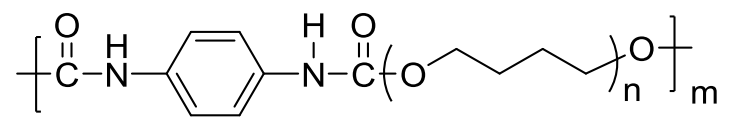

Under an argon atmosphere, to a flame-dried 25-mL two-neck round-bottomed flask placed in a pre-heated oil bath at $130{ }^{\circ} \mathrm{C}, 0.153 \mathrm{~g}(0.544 \mathrm{mmol})$ of DATA and $0.544 \mathrm{~g}(0.544$ mmol) of PTMEG and $0.00379 \mathrm{~g}(0.0272 \mathrm{mmol})$ of TBD were added. The reaction was monitored by IR spectroscopy. After $2 \mathrm{~min}$, the IR spectrum showed the characteristic peaks of polyurethane (amber in color). IR ( NaCl plate) $v_{\max } / \mathrm{cm}^{-1} 3304.2(\mathrm{~N}-\mathrm{H}$ stretching), $1727.2(\mathrm{C}=\mathrm{O}$ stretching), 1519.1 (NH bending and C-N stretching), 1219.8 (C-O), 1112 (C-O-C stretching).

$N$-acetoxyamide-terminated prepolymer $(\mathbf{P 3})$ 


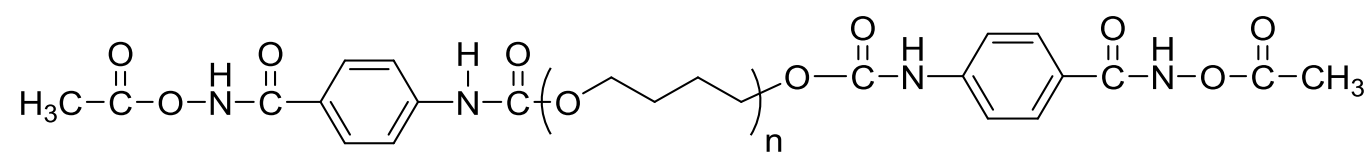

A flame-dried, $25 \mathrm{~mL}$, two-neck, round-bottomed flask was kept under argon for $15 \mathrm{~min}$ and placed in a pre-heated oil bath at $80{ }^{\circ} \mathrm{C}$. Then, $0.153 \mathrm{~g}(0.544 \mathrm{mmol})$ of DATA and $0.247 \mathrm{~g}$ (0.247 mmol) of PTMEG with $5 \mathrm{~mol} \%$ TBD (0.00379 g, $0.0272 \mathrm{mmol})$ were added to the flask. The reaction was monitored by IR spectroscopy. After $15 \mathrm{~min}$, prepolymer P3 was obtained as an amber liquid. IR ( $\mathrm{NaCl}$ plate) $v_{\max } / \mathrm{cm}^{-1} 3304.8(\mathrm{~N}-\mathrm{H}$ stretching), $1790.9(\mathrm{C}=\mathrm{O}$ stretching, from acetoxy) $1727.5(\mathrm{C}=\mathrm{O}$ stretching, from urethane), 1651.1 (amide $\mathrm{C}=\mathrm{O}), 1519$ ( $\mathrm{NH}$ bending and C-N stretching), 1219.4 (C-O), 1114.8 (C-O-C).

NCO-terminated prepolymer P1 from prepolymer P3<smiles>CC(C)(CCCCOC(C)(C)Nc1ccc([N+](=O)[O-])cc1)OC(=O)Nc1ccc([N+](=O)[O-])cc1</smiles>

Upon heating at $150{ }^{\circ} \mathrm{C}$, the formation of the resulting product was monitored and confirmed by IR analysis to be identical to P1. IR ( NaCl plate) $v_{\max } / \mathrm{cm}^{-1} 3306.8(\mathrm{~N}-\mathrm{H}$ stretching), 2152.9 ( $\mathrm{NCO}$ asymmetric stretching), 1727.1 ( $\mathrm{C}=\mathrm{O}$ stretching), 1517.1 ( $\mathrm{NH}$ bending and C-N stretching), 1220.9 (C-O stretching), 1110.9 (C-O-C stretching).

\section{Polyurethane P4}

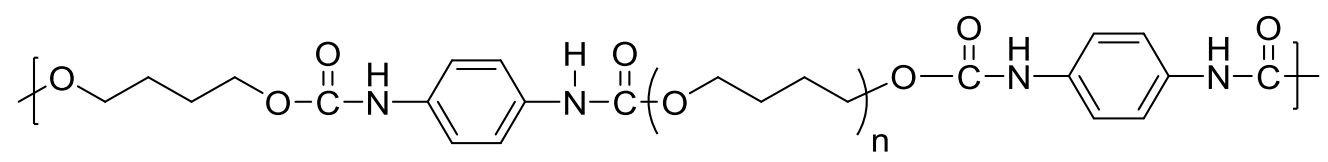

Under an argon atmosphere, $0.290 \mathrm{mmol}$ of 1,4-butanediol was added to a volumetric flask containing P1. After heating to $80{ }^{\circ} \mathrm{C}$ for $5 \mathrm{~min}$, the polyurethane $\mathbf{P 4}$ was obtained as an 
amber elastomeric product. IR ( $\mathrm{NaCl}$ plate) $\left.v_{\max } / \mathrm{cm}^{-1}\right) 3314$ (N-H stretching), 1729.9 and 1707 (C=O stretching), 1516.9 (NH bending and C-N stretching), 1219.8 (C-O stretching), 1051.4 (CO-C stretching).

Di(heptadecan-9-yl) 1,4-phenylenedicarbamate (1)

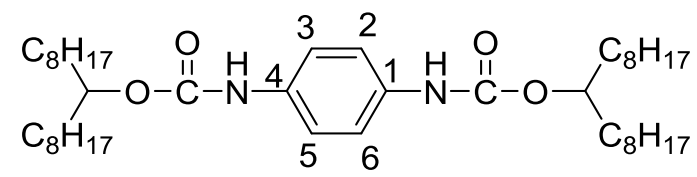

Under an argon atmosphere, in a flame-dried, $25 \mathrm{~mL}$, two-neck, round-bottomed flask $1.19 \mathrm{~g}(4.26 \mathrm{mmol})$ of DATA was dissolved in $1 \mathrm{~mL}$ of dioctyl terephthalate. The flask was placed in a pre-heated oil bath at $150{ }^{\circ} \mathrm{C}$. Continuous of IR analysis revealed that after 25 min the peak for $\mathrm{NCO}$ became very broad and the acetate $\mathrm{C}=\mathrm{O}$ peak became smaller. The temperature was then reduced from $150{ }^{\circ} \mathrm{C}$ to $90{ }^{\circ} \mathrm{C}$ and $2.19 \mathrm{~g}(8.52 \mathrm{mmol})$ of 9-heptadecanol was added. After 10 minutes, the NCO peak disappeared. The reaction continued for $1.5 \mathrm{~h}$ and then stopped. The reaction mixture was extracted three times by $\mathrm{CHCl}_{3}$. The solvent was evaporated under reduced pressure and the residue was purified by silica gel column chromatography using hexane and acetone (10\% acetone: hexane) as eluting solvents. The product was white in color with a melting point of $122{ }^{\circ} \mathrm{C}$ (40\% of yield). The work-up procedure was same for the base catalysed reactions and the obtained product yield was $50-52 \%$. IR ( $\mathrm{NaCl}$ plate) $v_{\max } / \mathrm{cm}^{-1} 3280.2$ ( $\mathrm{N}-\mathrm{H}$ stretching), 1694.5 (C=O stretching), 1551.2 ( $\mathrm{NH}$ bending and C-N stretching), 1237.5 (C-O stretching), 1086.8 (C-O-C stretching). ${ }^{1} \mathrm{H}$ NMR (300 MHz, $\left.\mathrm{CDCl}_{3}\right) \delta 7.34(\mathrm{~s}, 4 \mathrm{Ar}-\mathrm{H}), 6.50(\mathrm{~s}, 2 \mathrm{NH}), 4.84(\mathrm{~m}, 2 \mathrm{H}-\mathrm{CH}), 1.59\left(\mathrm{~m}, 8 \mathrm{H}_{-} \mathrm{CH}_{2}\right) 1.28(\mathrm{~m}, 24 \mathrm{H}-$ $\left.\mathrm{CH}_{2}\right), 0.89\left(\mathrm{~m}, 12 \mathrm{H}-\mathrm{CH}_{3}\right) .{ }^{13} \mathrm{C} \mathrm{NMR}\left(75 \mathrm{MHz}, \mathrm{CDCl}_{3}\right) \delta 153.70(\mathrm{C}=\mathrm{O}), 133.65$ (Ar-Cland $\left.\mathrm{C} 4\right)$, 
119.33 (Ar-C2, C3, C5 and C6), 75.62 (HC-O), 34.45, 31.77, 29.60, 25.06, 22.60- $\mathrm{CH}_{2}, 14.12$

$\left(\mathrm{CH}_{3}-\mathrm{C}\right)$. MS-ESI m/z: $[\mathrm{M}+\mathrm{Na}]^{+}$calculated for $\mathrm{C}_{42} \mathrm{H}_{76} \mathrm{~N}_{2} \mathrm{O}_{4} \mathrm{Na}$, 695.5700, Found, 695.5703 .

$N$-acetoxybenzamide

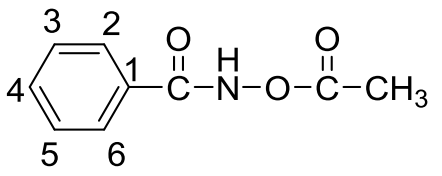

In a $50 \mathrm{~mL}$, three-neck, round-bottomed flask, $N$-hydroxy benzamide $(1.04 \mathrm{~g}, 7.56 \mathrm{mmol})$ was dissolved in $20 \mathrm{~mL}$ of $\mathrm{CH}_{2} \mathrm{Cl}_{2}$ and $7.56 \mathrm{mmol}(2 \mathrm{M}, 4.15 \mathrm{~mL})$ of an aqueous solution of $\mathrm{NaOH}$ was added to that mixture, followed by dropwise addition of acetic anhydride $(0.8 \mathrm{~mL}$, $8.32 \mathrm{mmol})$. The reaction mixture was stirred at room temperature and the progress of the reaction was monitored by TLC (hexane: acetone, 2:1). After 2 hours, the completion of the reaction was indicated by TLC. The reaction mixture was extracted with $\mathrm{CH}_{2} \mathrm{Cl}_{2}$ and the organic extracts were dried over anhydrous $\mathrm{MgSO}_{4}$. After removing the solvent by rotary evaporator at $30{ }^{\circ} \mathrm{C}, \mathrm{N}$-acetoxybenzamide was obtained as a white solid: $0.9899 \mathrm{~g}(73.0 \%$ of yield); m.p. $120-$ $121{ }^{\circ} \mathrm{C} .{ }^{26} \mathrm{IR}\left(\mathrm{NaCl}\right.$ plate) $v_{\max } / \mathrm{cm}^{-1} 3145$ (N-H stretching), 1792 (C=O stretching, from acetoxy) $1648\left(\mathrm{C}=\mathrm{O}\right.$ stretching, from amide), 1530 (NH bending and $\mathrm{C}-\mathrm{N}$ stretching). ${ }^{1} \mathrm{H}$ NMR (300 MHz, DMSO-d $\left.\mathrm{d}_{6}\right) \delta 12.40(\mathrm{~s}, 1 \mathrm{NH}), 7.81(\mathrm{~m}, 2 \mathrm{Ar}-\mathrm{H}), 7.61(\mathrm{~m}, 1 \mathrm{Ar}-\mathrm{H}), 7.53(\mathrm{~m}, 2 \mathrm{Ar}-\mathrm{H}), 2.25(\mathrm{~s}$, 3CH $\left.\mathrm{CH}_{3}-\mathrm{H}\right) .{ }^{13} \mathrm{C}$ NMR (75 MHz, DMSO-d $\left.\mathrm{d}_{6}\right) \delta$ 169.1(C=O), $165.0(\mathrm{C}(\mathrm{O}) \mathrm{O}), 132.7(\mathrm{Ar}-\mathrm{C} 1), 131.4$ (Ar-C4), 129.1 (Ar-C2), 127.8 (Ar-C3), $18.6\left(\mathrm{CH}_{3}-\mathrm{C}\right)$. MS (EI): Exact mass calculated for $\mathrm{C}_{9} \mathrm{H}_{9} \mathrm{NO}_{3}[\mathrm{M}]^{+}: 179.0600$, found: 176.1001 .

Butyl phenylcarbamate (2) 


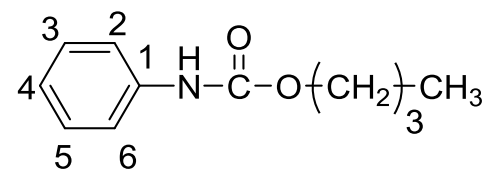

In a flame-dried, 2-neck, $25 \mathrm{~mL}$ round-bottomed flask, $(0.113 \mathrm{~g}, 0.630 \mathrm{mmol})$ of $\mathrm{N}$ acetoxybenzamide was dissolved in $1 \mathrm{~mL}$ of chlorobenzene. The solution was then heated at 120 ${ }^{\circ} \mathrm{C}$. After $1.5 \mathrm{~h}$, the $\mathrm{NCO}$ asymmetric stretching peak became intense and then $0.0467 \mathrm{~g}(0.630$ mmol) of $n$-butanol was added to the reaction mixture. After 15 minutes, IR spectrum showed the disappearance of the characteristic NCO peak and then the reaction stopped. The reaction mixture was then extracted three times with $\mathrm{CHCl}_{3}$ and the organic extracts were evaporated by rotary evaporator. Preparative TLC was performed on the crude material using a mixture of hexane and acetone $(1: 1, \mathrm{v} / \mathrm{v})$ as developing solvents. Three compounds were isolated as compounds 2, 3 and some unreacted starting materials. The urethane product $\mathbf{2}$ was obtained as a yellowish solid (35\% yield). m.p. $58{ }^{\circ} \mathrm{C}$. IR ( $\mathrm{NaCl}$ plate) $v_{\max } / \mathrm{cm}^{-1} 3310.6$ (N-H stretching), 1737.4 and $1707.7(\mathrm{C}=\mathrm{O}$ stretching), $1545.6(\mathrm{NH}$ bending and $\mathrm{C}-\mathrm{N}$ stretching) $1226.6(\mathrm{C}-\mathrm{O}$ stretching), 1070 (C-O-C stretching). ${ }^{1} \mathrm{H}$ NMR (300 MHz, DMSO-d 6 ) $\delta$ 7.40-7.56 (m, 4Ar-H), $7.07(\mathrm{~m}, 1 \mathrm{Ar}-\mathrm{H}), 6.63(\mathrm{~s}, 1 \mathrm{NH}), 4.19\left(\mathrm{~m}, 2 \mathrm{CH}_{2}-\mathrm{H}\right), 1.69\left(\mathrm{~m}, 2 \mathrm{CH}_{2}-\mathrm{H}\right) 1.45\left(\mathrm{~m}, 2 \mathrm{CH}_{2}-\mathrm{H}\right), 0.97$ $\left(\mathrm{m}, 3 \mathrm{CH}_{3}-\mathrm{H}\right) .{ }^{13} \mathrm{C}$ NMR $\left(75 \mathrm{MHz}, \mathrm{DMSO}-\mathrm{d}_{6}\right) \delta 153.713(\mathrm{C}=\mathrm{O}), 137.978(\mathrm{Ar}-\mathrm{C} 1), 129.048(\mathrm{Ar}-$ C3 and C5), 123.661 (Ar-C4), 118.618 (Ar-C2 and C6), 65.133, 30.710, $19.09\left(\mathrm{CH}_{2}-\mathrm{C}\right), 13.743$ $\left(\mathrm{CH}_{3}-\mathrm{C}\right) . \mathrm{MS}(\mathrm{EI})$ : Exact mass calculated for $\mathrm{C}_{11} \mathrm{H}_{15} \mathrm{NO}_{2}[\mathrm{M}]^{+}$: 193.1101, found: 193.1100 .

\section{1,3-Diphenylurea (3)}

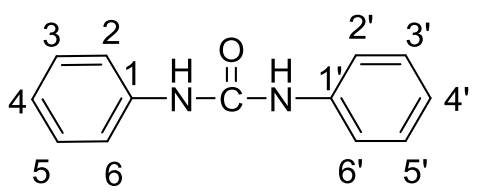


Compound 3 was isolated as yellow solid in $25 \%$ yield, m.p. approximately $220{ }^{\circ} \mathrm{C}$ decomposed. ${ }^{27} \mathrm{IR}\left(\mathrm{NaCl}\right.$ plate) $v_{\max } / \mathrm{cm}^{-1} 3321.5(\mathrm{~N}-\mathrm{H}$ stretching), $1649.8(\mathrm{C}=\mathrm{O}$ stretching), 1556.1 (NH bending and C-N stretching). ${ }^{1} \mathrm{H}$ NMR (300 MHz, DMSO-d 6 ) $\delta 8.67$ (s, 2NH) 7-8 $(\mathrm{m}, 10 \mathrm{Ar}-\mathrm{H}) .{ }^{13} \mathrm{C}$ NMR $\left(75 \mathrm{MHz}, \mathrm{DMSO}-\mathrm{d}_{6}\right) \delta 152(\mathrm{C}=\mathrm{O}), 140(\mathrm{Ar}-\mathrm{C} 1, \mathrm{C} 1$ '), $130(\mathrm{Ar}-\mathrm{C} 3, \mathrm{C} 5$ and C3', C5'), 123 (Ar-C4, C4'), 119 (Ar-C2, C6 and C2', C6'). MS (EI): Exact mass was found: 212.1001 for the formula, $\mathrm{C}_{13} \mathrm{H}_{12} \mathrm{~N}_{2} \mathrm{O}$. 


\subsection{References}

1. Gogoi, R.; Alam, M. S.; Khandal, R. K. Engineering Res. Tech. 2014, 3, 1404.

2. Majoros, L.; Dekyser, B.; Hoogenboom, R.; Fijten, M. W. M.; Haucourt, N.; Schubert, U. S. Polym. Chem. 2009, 47, 3729.

3. Smith, A. K.; Goddard, R. J.; Paulsen, E. J. L. US patent 2005/006884904B2, 2005.

4. Unverferth, M.; Kreye, O.; Prohammer, A.; Meier, M. A.R. Macromol. Rapid Commun. 2013, 34, 1569.

5. Heintz, A. M.; Duffy, D. J.; Hsu, S. L. Macromolecules 2003, 36, 2695.

6. Gogoi, R.; Alam, M. S.; Khandal, R. K. Engineering Res. Tech. 2014, 3, 118.

7. Bauer, L; Exner, O. Angew. Chem. Internat. Edit.1974, 13, 376.

8. Deepa, P; Jayakannan, M. Polym. Chem. 2007, 45, 2351.

9. PPDI for high-performance polyurethanes, 1992, from https://www.thefreelibrary.com/+for+high+performance+polyurethanes.-a012146965.

10. Codd, R. Coord. Chem. Rev. 2008, 252, 1387.

11. Matlin, S. A.; Sammes, P. G.; Upton, R. M. J. Chem. Soc. Perkin Trans. 1979, 1, 2481.

12. Yamaye, M.; Nago, Y.; Sasaki, M.; Tsuru, T.; Mukae, K.; Yoshinaga, T.; Murayama, R.; Tahara, C. Polym. Degrad. Stability 2006, 91, 2014.

13. Hynes, J. B. J. Med. Chem. 1970, 13, 1235.

14. Wang, j.; Zhang, G.; Liu, Z.; Gu, X.; Yan, Y.; Zhang, C.; Xu, Z.; Zhao, Y.; Fu, H.; Zhang, D. Tetrahedron 2013, 69, 2687.

15. Ghosh, K. K.; Tamrakar, P. Indian J. Chem. A, 2003, 42, 1081.

16. Smith, M. B.; March, J. March's Advanced Organic Chemistry: Reactions, Mechanisms, and Structure, Published by John Wiley \& Sons, Inc., New Jersey, 2007, Part 2, p1559. 
17. Lossen, W. Liebigs Ann. Chem. 1872, 161, 347; 1877, 186, 1; 1889, 252, 170. 1894, 281, 169.

18. Bauer, L.; Exner, O.; Angew. Chem. int. Ed. 1974, 13, 376.

19. Cherest, M.; lusinchi, X. Tetrahedron Lett. 1989, 30, 715.

20. Guimond, N.; Gorelsky, S. I.; Fagnou, K. J. Am. Chem. Soc. 2011, 133, 6449.

21. Webb, N. J.; Marsden, S. P.; Raw, Org. Lett. 2014, 16, 4718.

22. Uehara, K.; Fukaya, K.; Mizuno, N. Angew. Chem. Int. Ed. 2012, 51, 7715.

23. Pathak, G.; Das, D.; Rokhum, L. RSC Adv. 2016, 6, 93729.

24. Eyster, E. H.; Gillette, R. H. J. Chem. Phys. 1940, 8, 36.

25. Jiao, L.; Xiao, H.; Wang, Q.; Sun, J. Polym. Degrad. Stability, 2013, 98, 2687.

26. Ghosh, H.; Patel, B. K. Org. Biomol. Chem. 2010, 8, 384.

27. Lebel, H.; Leogane, O. Org. Lett. 2006, 8, 5717. 


\section{Chapter 3 Design, Synthesis and Characterizations of Pendent NCO-containing Polymethacrylates}

\subsection{Introduction}

A pendent $\mathrm{NCO}$-containing polymer contains the isocyanato $(\mathrm{NCO})$ groups on the side chains. The NCO-containing polymers can react with alcohols and amines to yield the modified polymers for certain applications. ${ }^{1}$ Such a reactive polymer is in demand both in academic research and industry. ${ }^{2}$ The NCO-containing polyacrylates and polymethacrylates are typically synthesized by polymerization with isocyanate containing monomer, ${ }^{3,4}$ modification of the initiator $^{5,6}$ and polymer modification. ${ }^{7,8}$ Since the isocyanate functional groups are extremely moisture sensitive, a care should be taken to keep the reaction system dry. A glove box may be

used for conducting the reactions and for storage monomers as well. ${ }^{7,9}$ There are only two commercially available NCO-containing monomers useful for free radical polymerization: dimethyl meta-isopropenyl benzyl isocyanate (TMI) and 2-isocyanatoethyl methacrylate (IEM) (Figure 3.1). TMI-functionalized polymers were first introduced by Dexter et. al. ${ }^{10}$ and then a number of follow-up studies have been reported. ${ }^{11}$ IEM is widely used due to its ability to undergo a free-radical polymerization under mild conditions with a wide variety of vinyl monomers. $^{12-14}$

Benzoyl azide ${ }^{7}$ or cinnamoyl azide ${ }^{15}$ has been incorporated into a monomer for polymerization and the acyl azide group of the polymer can be transformed into the isocyanate via Curtius rearrangement, which then reacts with an alcohol (Scheme 3.1). This type of monomers is synthesized from the reaction of the corresponding acid with triethylamine and ethyl chloroformate in the presence of toxic sodium azide. 


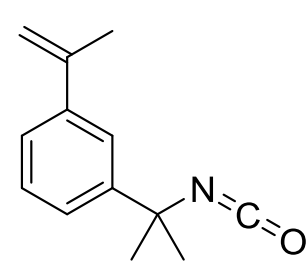

Dimethyl meta-isopropenyl benzyl isocyanate (TMI)

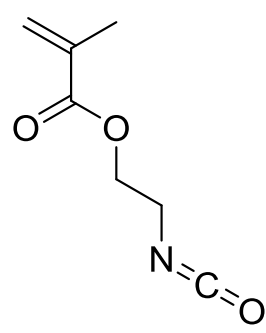

2-isocyanatoethyl methacrylate (IEM)

Figure 3.1 Isocyanate-functionalized monomers
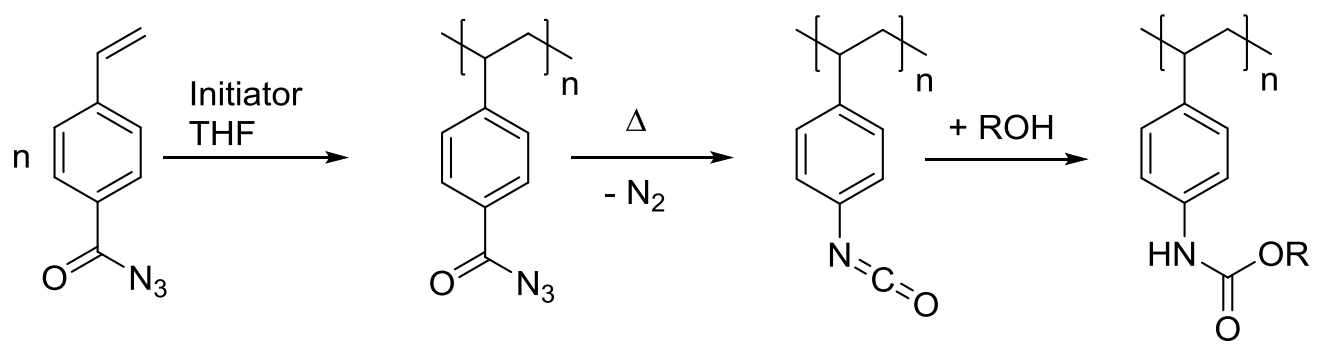

Scheme 3.1 Synthesis of the pendent NCO-containing polystyrene using 4-vinylbenzoyl azide via Curtius rearrangement

Polymerization of NCO-containing monomers has some limitations due to the high reactivity of NCO group. The crosslinking reaction may take place due to the formation of the urea bond as a result of exposure to the moisture, ${ }^{16}$ or formation of a cyclic trimer of the $\mathrm{NCO}$ groups at high temperature. $^{17}$

Poly(methyl methacrylate) (PMMA) is a well-known polymer with high transmittance, good mechanical strength and processability. ${ }^{18}$ PMMA is also subject to intense research for structural modifications. ${ }^{19}$ Therefore, we intend to explore a simple and convenient approach to the synthesis of latent NCO-containing polymethacrylates, based on the previous studies on the chemistry of the $\mathrm{N}$-acetoxyamide (AA) group (Chapter 2). Since the AA group is a latent NCO 
group, we can demonstrate this new approach by incorporating $N$-acetoxymethacrylamide (AMAA) into PMMA by polymerization of AMAA with methyl methacrylate (MMA).

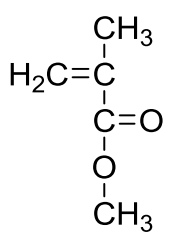

Methyl methacrylate (MMA)

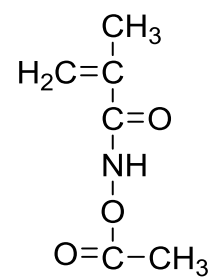

N-acetoxymethacrylamide (AMAA)

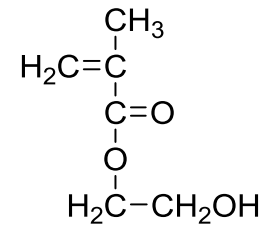

2-hydroxyethyl methacrylate (HEMA)

Figure 3.2 Methyl methacrylate, $N$-acetoxymethacrylamide and 2-hydroxyethyl methacrylate

Herein we designed two series of binary and ternary methacrylate copolymers containing the latent NCO group. AMAA is used as a latent isocyanate comonomer, MMA is a major monomer in polymerization, and 2-hydroxyethyl methacrylate (HEMA) is also used as a comonomer (Figure 3.2). The hydroxyl group in HEMA can react with the resulting NCO group in the polymer, leading to polymer crosslinking. The synthesized copolymers were characterized by the IR, ${ }^{1} \mathrm{H}$ NMR, ${ }^{13} \mathrm{C}$ NMR, TGA, DSC, and GPC. In addition, thermal conversion of the AA to NCO groups in these copolymers was studied by IR spectroscopy.

\subsection{Synthesis and characterizations of $N$-hydroxymethacrylamide}

The synthetic route to $N$-hydroxymethacrylamide (NHMA) is shown in Scheme 3.2. The reaction of acrylate with hydroxylamine was used to prepare the corresponding hydroxamic acid. ${ }^{20}$ However, the Michael addition reaction could occur at the vinyl group, leading to the formation of side products. Although the use of acid chloride can solve this problem, the product yield is rather low. ${ }^{21}$ In our work, NHMA was synthesized from MMA with hydroxylamine. In order to prevent the hydrolysis of the ester group in MMA, MMA was added to the mixture of 
sodium hydroxide and hydroxylamine hydrochloride at $0-5{ }^{\circ} \mathrm{C}$. The reaction continued for 24 hours at room temperature and after work up, NHMA was obtained as yellowish semi-solid in quantitative yield.

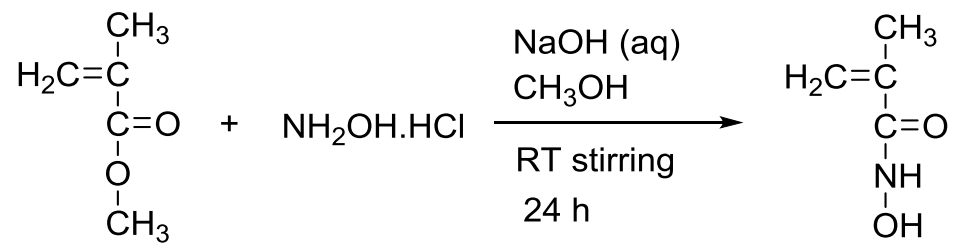

Methyl methacrylate

$\mathrm{N}$-hydroxymethacrylamide

Scheme 3.2 Synthesis of $N$-hydroxymethacrylamide

$N$-hydroxymethacrylamide (NHMA) was fully characterized by the IR, ${ }^{1} \mathrm{H}$ NMR, ${ }^{13} \mathrm{C}$ NMR, and mass spectrometry. All the spectra are given in "Appendix B". The IR spectrum (Figure S3.1) of NHMA displays the characteristic $\mathrm{HN}-\mathbf{C}=\mathbf{O}$ band at $1657 \mathrm{~cm}^{-1}$, as well as the $\mathrm{HN}-\mathrm{C}=\mathrm{O}$ bending and $\mathrm{C}-\mathrm{N}$ stretching band at $1526 \mathrm{~cm}^{-1}$. The broad peak at $3235 \mathrm{~cm}^{-1}$ is a stretching band for $\mathrm{NH}$ and $\mathrm{OH}$. The ${ }^{1} \mathrm{H}$ NMR spectrum (Figure S3.2) of NHMA shows broad singlet peaks at $10.71 \mathrm{ppm}$, and $8.82 \mathrm{ppm}$, which are due to $\mathrm{NH}$ and $\mathrm{OH}$ protons, respectively. The two singlet peaks at $5.56 \mathrm{ppm}$ and $5.28 \mathrm{ppm}$ are due to methylene protons and another singlet at $1.83 \mathrm{ppm}$ for methyl protons, respectively. The ${ }^{13} \mathrm{C}$ NMR spectrum (Figure S3.3) of NHMA shows peaks at $165.75,138.08,119.46,18.89 \mathrm{ppm}$, assigned to amide $\mathrm{C}=\mathrm{O}$, methylene carbons, and methyl carbon, respectively. Mass spectrum (Figure S3.4) shows a molecular ion peak at $\mathrm{m} / \mathrm{z} 101$, which corresponds to the desired compound.

\subsection{Synthesis and Characterizations of $\mathrm{N}$-acetoxymethacrylamide}

The target monomer AMAA was synthesized by acylation of NHMA in the presence of a molar equivalent of $\mathrm{NaOH}$ (Scheme 3.3). The reaction mixture was stirred for $24 \mathrm{~h}$ at room 
temperature and AMAA was obtained as a dense, colorless liquid. With an excess of $\mathrm{NaOH}$, further, acylation took place, resulting in an $N$-substituted by-product.

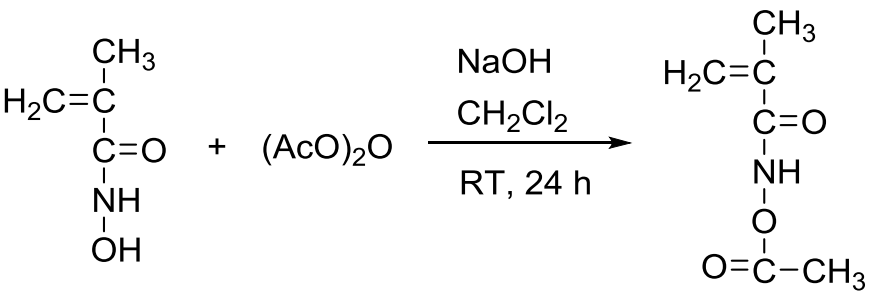

N-hydroxymethacrylamide

N-acetoxymethacrylamide

Scheme 3.3 Synthesis of $N$-acetoxymethacrylamide

AMAA was fully characterized by the IR, ${ }^{1} \mathrm{H}$ NMR, ${ }^{13} \mathrm{C}$ NMR, and mass spectrometry. The IR spectrum (Figure 3.3) displays $\mathrm{O}-\left(\mathrm{CH}_{3}\right) \mathbf{C}=\mathbf{O}$ stretching band at $1795 \mathrm{~cm}^{-1}$ and $\mathrm{NH}$ stretching band at $3205 \mathrm{~cm}^{-1}$, which are the characteristic peaks for acetoxy $\mathrm{C}=\mathrm{O}$ and amide $\mathrm{NH}$. There is no peak at $3235 \mathrm{~cm}^{-1}$, indicating that all $\mathrm{OH}$ groups had been converted to ester and an NH band separated out. The ${ }^{1}$ H NMR spectrum (Figure 3.4) of AMAA shows a new singlet peak at $2.17 \mathrm{ppm}$, indicating the presence of $\mathbf{C H}_{3}-\mathrm{C}=\mathrm{O}$. There is no peak at $8.82 \mathrm{ppm}$, indicating all the $\mathrm{OH}$ groups took part in nucleophilic substitution reaction and produced AMAA. The NH peak was shifted downfield to $11.79 \mathrm{ppm}$ from $10.71 \mathrm{ppm}$ due to the electron withdrawing nature of acetoxy group. The methylene proton and ester $\mathrm{CH}_{3}$ proton ratio in AMAA is exactly 1:1:3, indicating that there was no nucleophilic substitution reaction between amide $\mathrm{NH}$ and acetic anhydride. The ${ }^{13} \mathrm{C}$ NMR spectrum (Figure S3.5) of AMAA shows two peaks at 169.01 and 165.96 ppm, indicating the $\mathrm{O}-\left(\mathrm{CH}_{3}\right) \mathbf{C}=\mathbf{O}$ and $\mathrm{HN}-\mathbf{C}=\mathbf{O}$ groups. The methylene carbons are at $137.00 \mathrm{ppm}, 121.73 \mathrm{ppm}$ and methyl carbon at $18.66 \mathrm{ppm}$, respectively. MS (ESI, DMSO/CH$\left.{ }_{3} \mathrm{OH}\right)$ : Exact mass calculated for $\mathrm{C}_{6} \mathrm{H}_{9} \mathrm{NO}_{3}[\mathrm{M}+\mathrm{H}]^{+}:$144.0600, found: 144.0739 (Figure S3.6) 


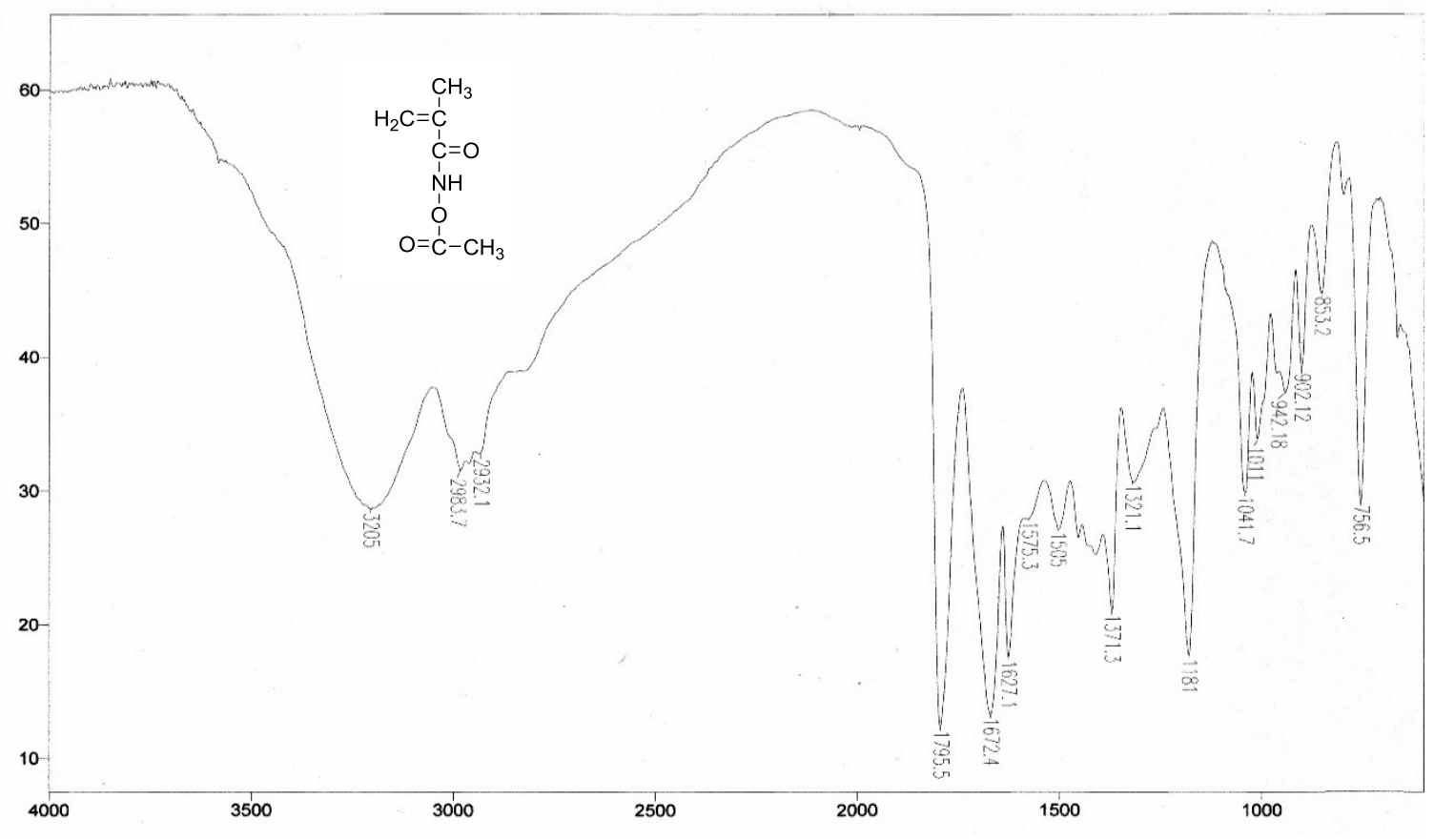

Figure 3.3 IR spectrum of $N$-acetoxymethacrylamide

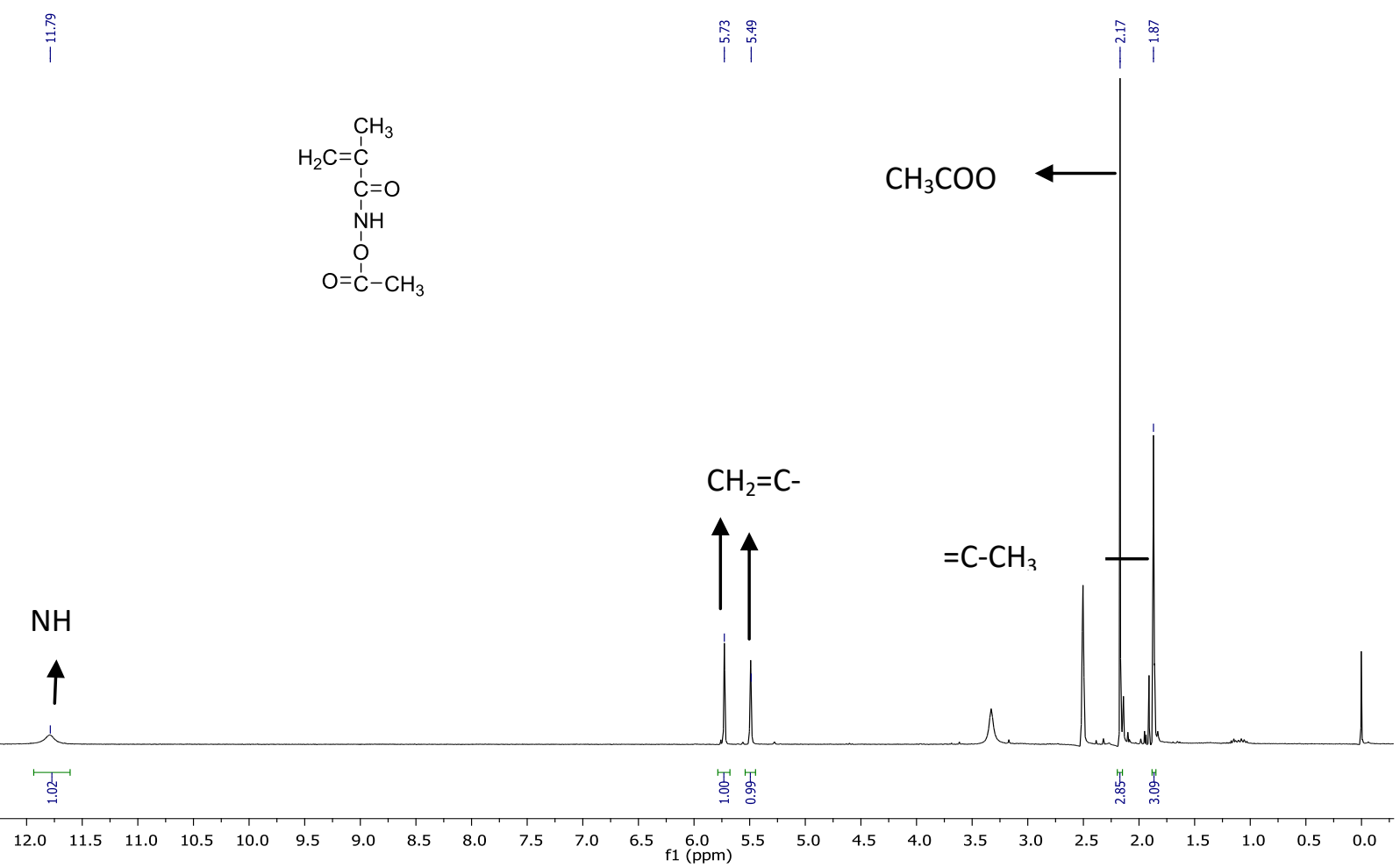

Figure $3.4{ }^{1} \mathrm{H}$ NMR spectrum $\left(300 \mathrm{MHz}, \mathrm{DMSO}-\mathrm{d}_{6}\right)$ of $\mathrm{N}$-acetoxymethacrylamide 


\subsection{Synthesis and characterizations of MMA-AMAA copolymers}

A series of MMA-AMAA copolymers (P5-P7) were synthesized by free radical polymerization of MMA and AMAA with various molar ratios in THF at $55{ }^{\circ} \mathrm{C}$ for 24 hours (Scheme 3.4). Copolymers were isolated by precipitation in methanol or hexane.

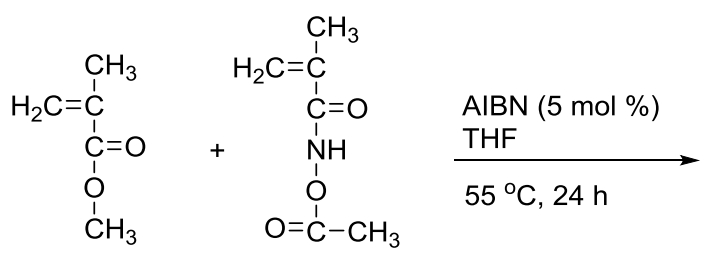

MMA
AMAA

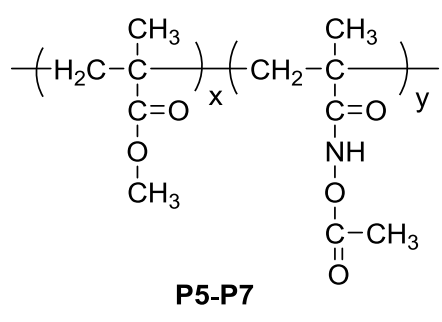

P5-P7

Scheme 3.4 Synthesis of MMA-AMAA copolymers P5-P7

Each of copolymers P5-P7 was fully characterized by the IR, ${ }^{1} \mathrm{H}$ NMR, ${ }^{13} \mathrm{C}$ NMR, TGA, DSC, and GPC. The IR spectra (Figure 3.5b-d) shows a peak at $1797 \mathrm{~cm}^{-1}$ for P5-P7, which is due to acetoxy $\mathrm{C}=\mathrm{O}$ of AMAA (Figure 3.5a). The peak intensity at $1797 \mathrm{~cm}^{-1}$ increases with an increase of the amount of AMAA. The ratios of AMAA in feed and in copolymers P5-P7 are represented in Table 3.1. The calculated ratio was obtained by comparing the peak intensity at $1797 \mathrm{~cm}^{-1}$ with a standard mixture (Figure 3.5e) of 9:1 molar ratio of PMMA to AMAA. With increasing the feed in ration the calculated ratio increase. The ${ }^{1} \mathrm{H}$ NMR spectra (Figure 3.6b-d) shows two new peaks at 9.43 and 2.21 ppm for P5-P7, corresponding to $\mathrm{NH}$ and $\mathrm{O}=\mathrm{C}-\mathrm{CH}_{3}$ (Figure 3.6a) of AMAA. The NH peak shifted upfield to $9.43 \mathrm{ppm}$ from $11.79 \mathrm{ppm}$ due to the less polar solvent, $\mathrm{CDCl}_{3}$. Figure 3.6 shows the peak intensity at 2.21 increasing from $\mathbf{P 5}$ to $\mathbf{P 7}$, indicating that the molar ratio of AMAA in polymer correlates to the feed ratio. Two singlet peaks at $5.73 \mathrm{ppm}$ and $5.49 \mathrm{ppm}$ from methylene protons are shifted to $1.82-1.90 \mathrm{ppm}$ as a broad peak, typical for a polymer. The singlet peak at $3.60 \mathrm{ppm}$ is from the $\mathrm{CH}_{3}-\mathrm{O}-\mathrm{C}=\mathrm{O}$ moiety of 
MMA, indicating the formation of the copolymer with AMAA. The ${ }^{13} \mathrm{C}$ NMR spectra (Figure S3.7, S3.11, S3.15) show three new peaks at 178.11, 177.81 and 176.94 ppm for P5-P7 from $\mathrm{C}=\mathrm{O}$ of acetoxy, $\mathrm{C}=\mathrm{O}$ of MMA and $\mathrm{NHC}=\mathrm{O}$ of AA. Decomposition temperatures $\left(\mathrm{T}_{\mathrm{d}}\right)$ for P5-P7 are presented in Table 3.2 and the thermograms are given in Appendix B (Figures S3.8, S3.12, and S3.16). All the copolymers display three-stage decomposition temperatures. From TGA data in Table 2, it is noticeable that with an increase of the molar ratio of AMAA in copolymers, the onset temperatures for decomposition gradually decrease. The first onset relates to the conversion of AA group to NCO group. The glass transition $\left(\mathrm{T}_{\mathrm{g}}\right)$ temperatures of P5-P7 were measured by differential scanning calorimetry (DSC) and the thermograms are presented in Figures S3.9, S3.13 and S3.17. The $\mathrm{T}_{\mathrm{g}}$ values of P5-P7 are 129.48, 122.71, $121.78{ }^{\circ} \mathrm{C}$, respectively. The $T_{g}$ decreases with increasing molar ratio of AMAA in copolymers. Since AMAA group is large enough to force the polymer chains apart, decrease intermolecular attraction. This result increasing more free volume to facilitate the segment movement, thus leading to a decrease in $\mathrm{T}_{\mathrm{g}}{ }^{22}$ However, the glass transition is affected by a number of factors, including heating rate, ageing history, morphology and molecular weight. Copolymers P5-P7 are of high molecular weights (Figures S3.10, S3.14 S3.18) with a polydispersity index from 1.71 to 2.65 .

Table 3.1 The onset temperatures for decomposition of copolymers P5-P7.

\begin{tabular}{|c|c|c|c|}
\hline Polymer & $1^{\text {st }}$ onset temperature $\left({ }^{\circ} \mathrm{C}\right)$ & $2^{\text {nd }}$ onset temperature $\left({ }^{\circ} \mathrm{C}\right)$ & $3^{\text {rd }}$ onset temperature $\left({ }^{\circ} \mathrm{C}\right)$ \\
\hline P5 & 170.17 & 269.68 & 322.27 \\
\hline P6 & 154.17 & 251.00 & 310.13 \\
\hline $\mathbf{P 7}$ & 144.47 & 245.69 & 305.58 \\
\hline
\end{tabular}




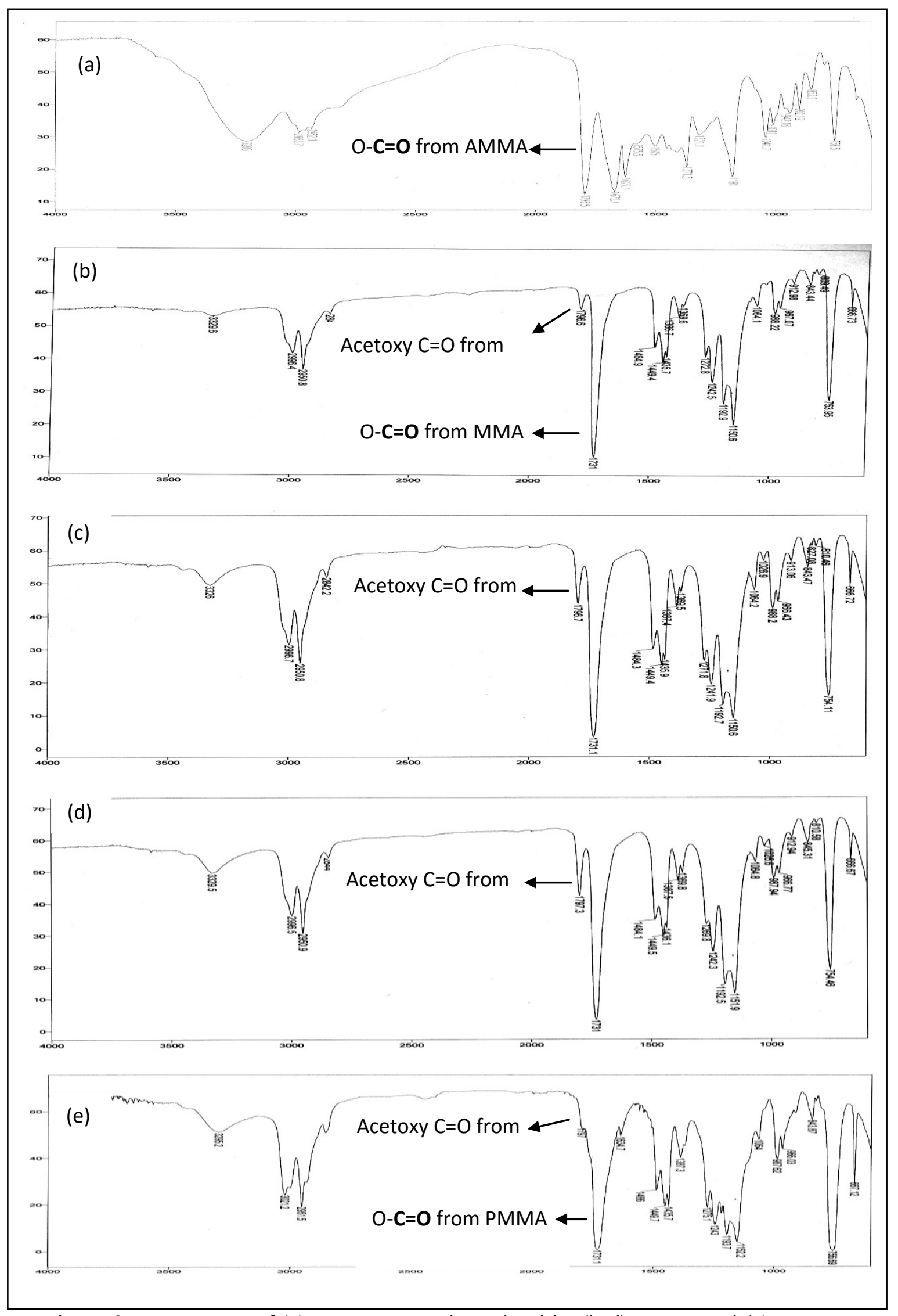

Figure 3.5 IR spectra of (a) $N$-acetoxymethacrylamide, (b-d) P5-P7 and (e) PMMA 


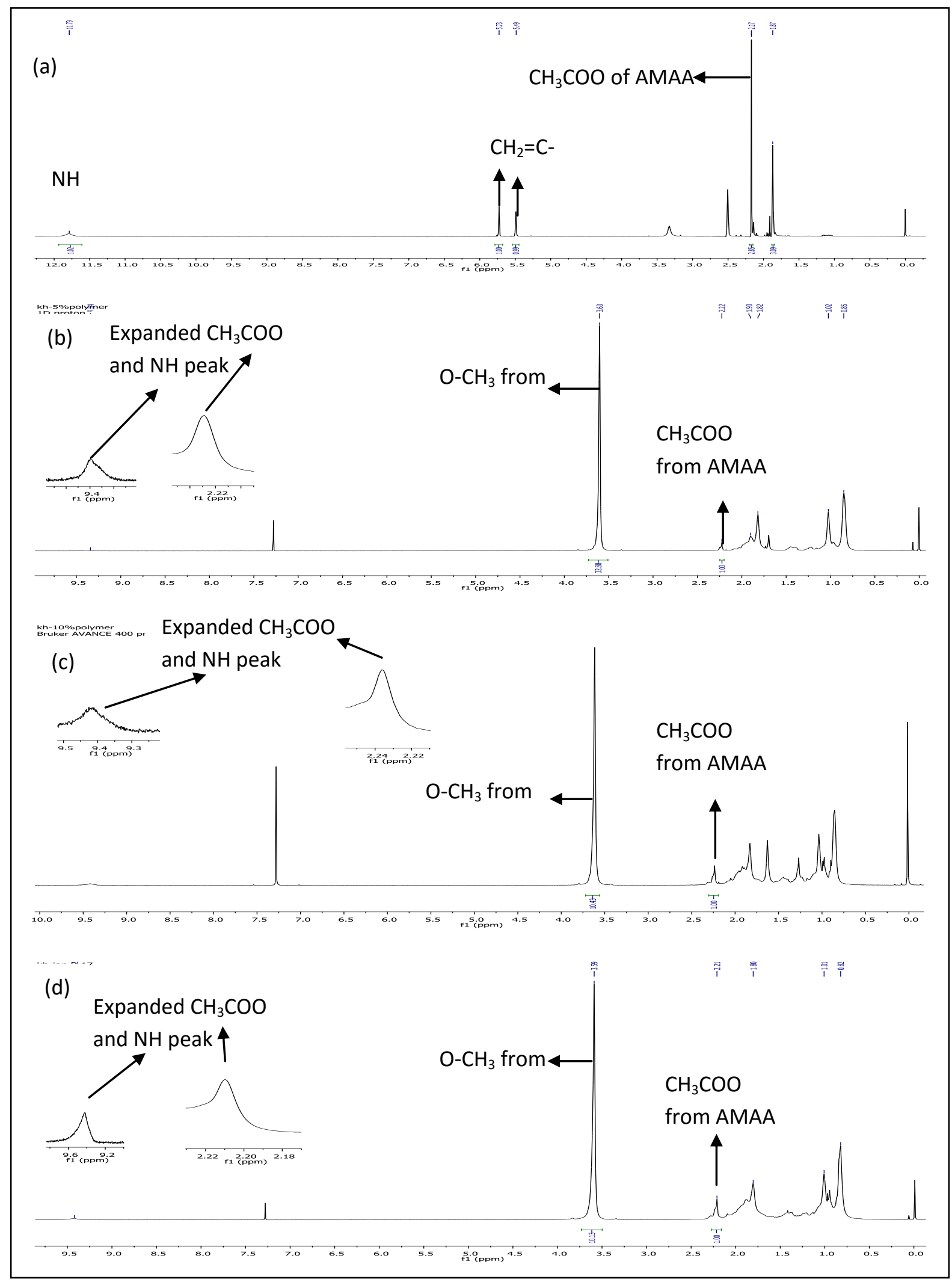

Figure $3.6{ }^{1} \mathrm{H}$ NMR spectra $\left(300 \mathrm{MHz}, \mathrm{CDCl}_{3}\right)$ of (a) $\mathrm{N}$-acetoxymethacrylamide (b-d) and P5-P7 
Table 3.2 The calculated and obtained ratios of AMAA in polymers (from IR spectra)

\begin{tabular}{|c|c|c|}
\hline Polymer & Ratio of AMAA in feed (mol \%) & Ratio of AMAA in copolymer \\
\hline P5 & 5 & 4.5 \\
\hline P6 & 10 & 11.9 \\
\hline P7 & 20 & 13.5 \\
\hline
\end{tabular}

\subsection{Conversion of $\mathrm{N}$-acetoxyamide to NCO in MMA-AMAA copolymers}

From DSC and TGA data, we conclude that at the temperatures between $129^{\circ} \mathrm{C}$ and 144 ${ }^{\circ} \mathrm{C}$, conversion of copolymers P5-P7 to analogous NCO-containing copolymers P8-P10 is feasible. Thus, the polymer transformation was studied at $110^{\circ} \mathrm{C}$ and $130^{\circ} \mathrm{C}$, respectively.

Polymer transformation at $110-130{ }^{\circ} \mathrm{C}$

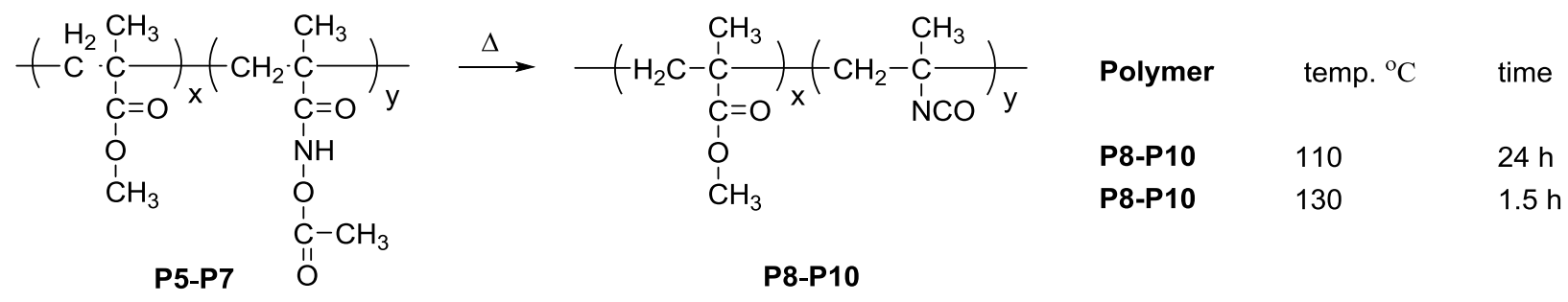

Scheme 3.5 Conversion of acetoxyamide to NCO in copolymers at $110-130{ }^{\circ} \mathrm{C}$

Copolymers P8-P10 were obtained by heating P5-7 in the powder form at $130{ }^{\circ} \mathrm{C}$ for 1.5 hours (Scheme 3.5). By IR analysis, the NCO peak appeared faster for P10 due to a higher content of AMAA, whereas relatively slow for P8. The conversion from AA to NCO was complete within 1.5 hours. The IR spectrum (Figure 3.7) shows a new peak at 2261 to $2263 \mathrm{~cm}^{-1}$ for NCO after 1.5 hours. There is no peak at $1795 \mathrm{~cm}^{-1}$ and $3205 \mathrm{~cm}^{-1}$, indicating that the AA group is fully converted to NCO. At $110^{\circ} \mathrm{C}$, transformation of P5-P7 to P8-P10 was also 
successful but required $24 \mathrm{~h}$ for completion. Thus, heating at $130{ }^{\circ} \mathrm{C}$ is the best for conversion of the AA to $\mathrm{NCO}$ in polymethacrylates.

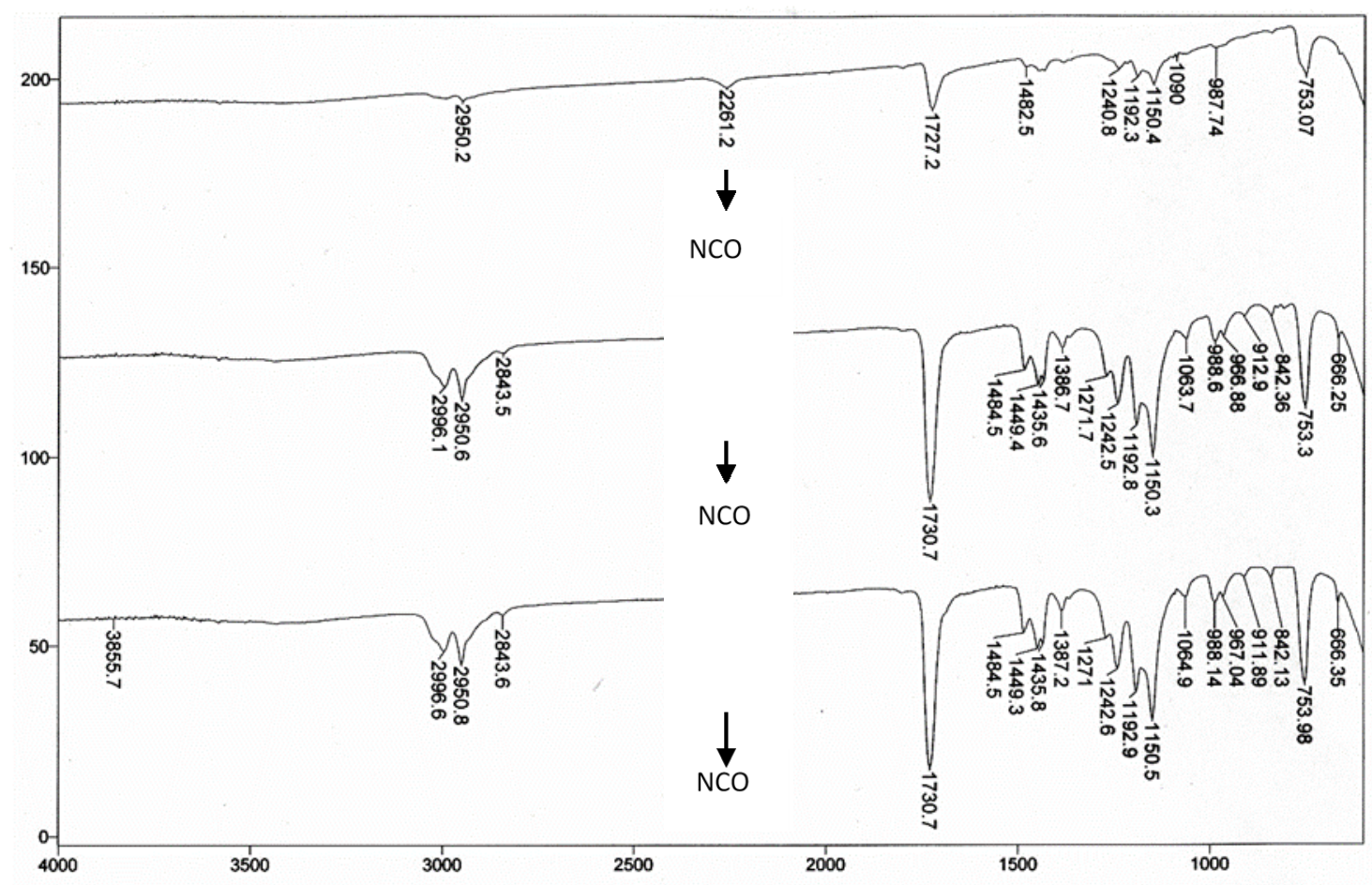

Figure 3.7 IR spectra of copolymers P8-P10

\section{Reaction of NCO-containing polymethacrylate with disperse red 1}

To further confirm the presence of reactive NCO group in copolymers P8-P10, we treated these NCO-containing polymers with a red dye called disperse red 1 (DR1). The nucleophilic addition reaction was carried out between polymer P8 and DR1 in presence of 5 mol \% DBTDL as catalyst at room temperature and as well $60{ }^{\circ} \mathrm{C}$. The reaction indeed took place between the NCO moiety of P8 and DR1 to afford red-colored polymer P11 (Scheme 3.6). Since DR1 is soluble in methanol, polymer P11 was isolated by precipitation into methanol to get rid of excess DR1. Polymer P11 is soluble in chloroform and characterized by the IR and ${ }^{1} \mathrm{H}$ NMR spectroscopy. 


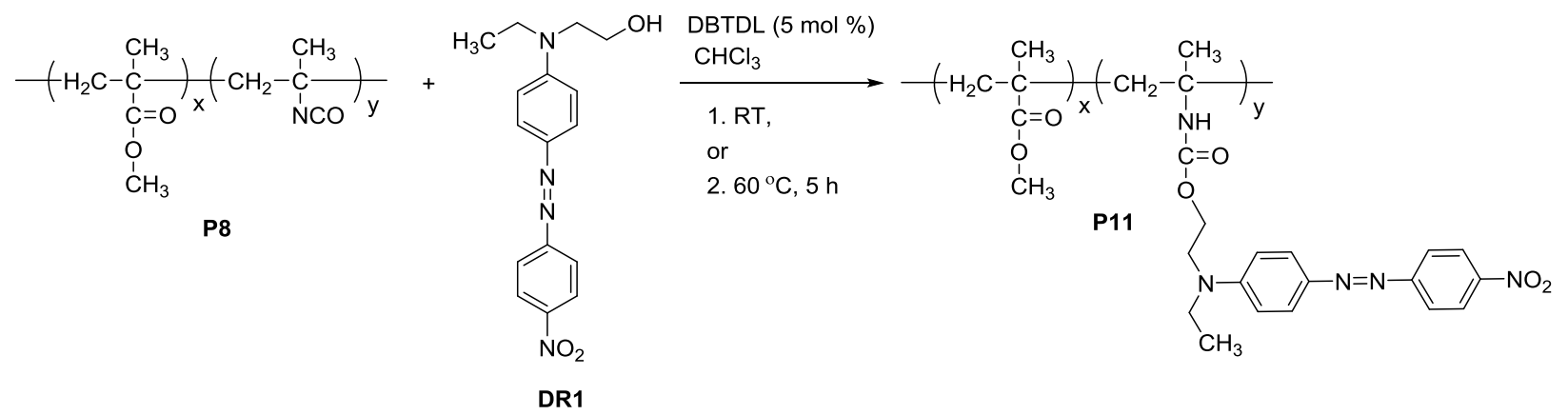

Scheme 3.6 Reaction of $\mathbf{P 8}$ and DR1 to yield P11

The IR spectrum (Figure 3.8a) shows the peak at $2261 \mathrm{~cm}^{-1}$, which is characteristic for NCO moiety of polymer P8. There is no peak (Figure 3.8b) at $2261 \mathrm{~cm}^{-1}$, indicating that all the $\mathrm{NCO}$ groups reacted with the $\mathrm{OH}$ group of DR1. The new peaks at 1602,1502 and $1340 \mathrm{~cm}^{-1}$ are assigned to the aromatic $\mathrm{C}=\mathrm{C}$, asymmetric and symmetric $\mathrm{NO}_{2}$, respectively, indicating the presence of $\mathrm{DR} 1$. The urethane $\mathrm{C}=\mathrm{O}$ was not possible to identify separately in the IR spectrum (Figure 3.8b) due to the overlap of the $\mathrm{C}=\mathrm{O}$ from MMA. ${ }^{23}$ The ${ }^{1} \mathrm{H}$ NMR spectrum (Figure 3.9) of $\mathbf{P 1 1}$ shows three new peaks in the aromatic region at 8.35, 7.94 and $6.8 \mathrm{ppm}$, as a result of DR1 chemically bounded to the polymer. Figure 3.10 represents the absorption spectra of precursor polymer P5, NCO-pendent polymer P8, DR1 and polymer P11 chemically grafted with DR1. Polymers $\mathbf{P 5}$ and $\mathbf{P 8}$ have weak absorption below $300 \mathrm{~nm}$ because of $\mathrm{n}-\pi^{*}$ transition. DR1 shows two absorption maxima at $478 \mathrm{~nm}$ for the $\pi-\pi^{*}$ transition and at $285 \mathrm{~nm}$ due to the $\mathrm{n}-$ $\pi^{*}$ transition. Polymer P11 shows the similar absorption to DR1 with two absorption maximal peaks at 478 and $285 \mathrm{~nm}$, indicating the successful grafting of DR1 onto polymer P8. 


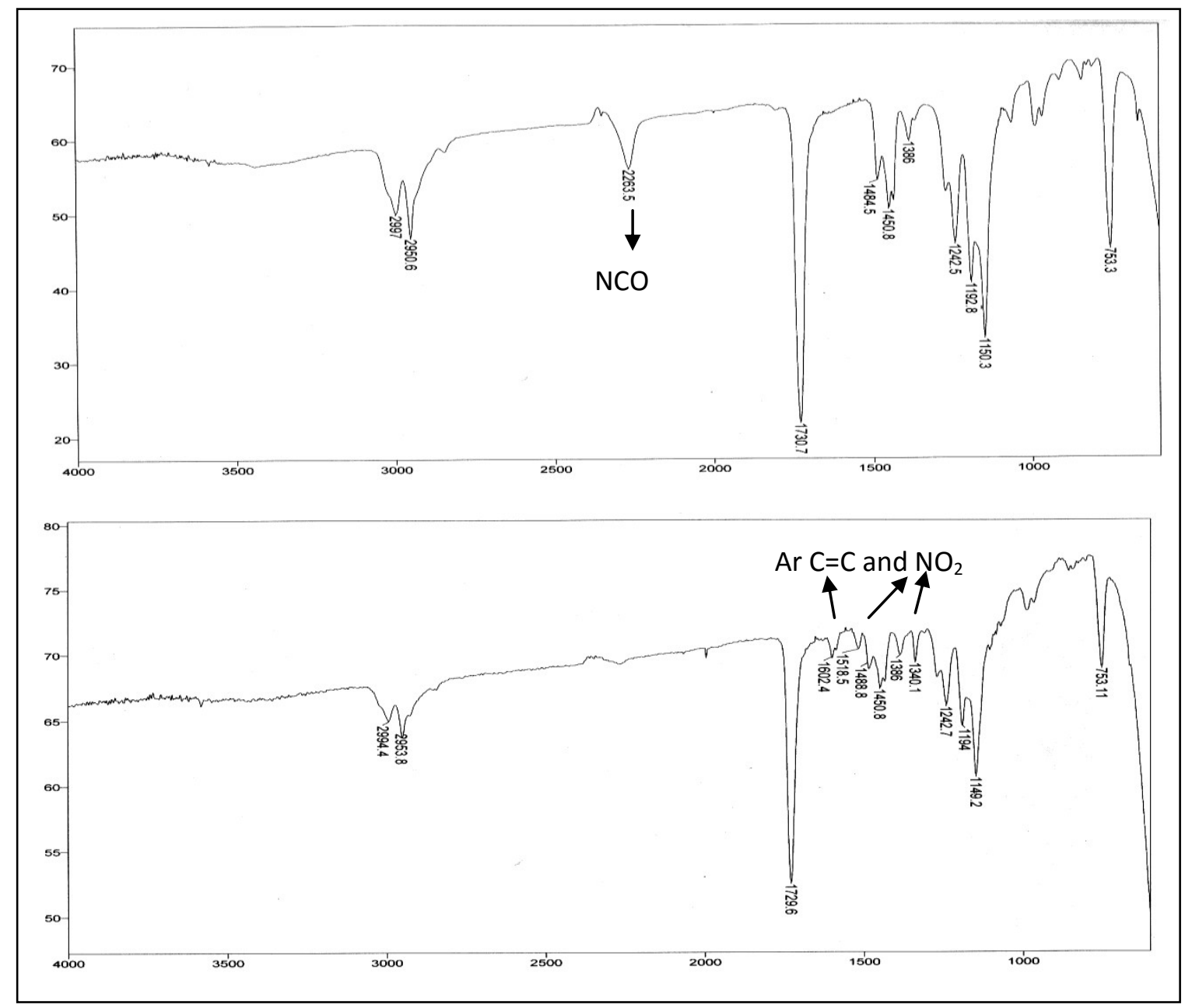

Figure 3.8 IR spectra of copolymers (a) P8 and (b) P11 


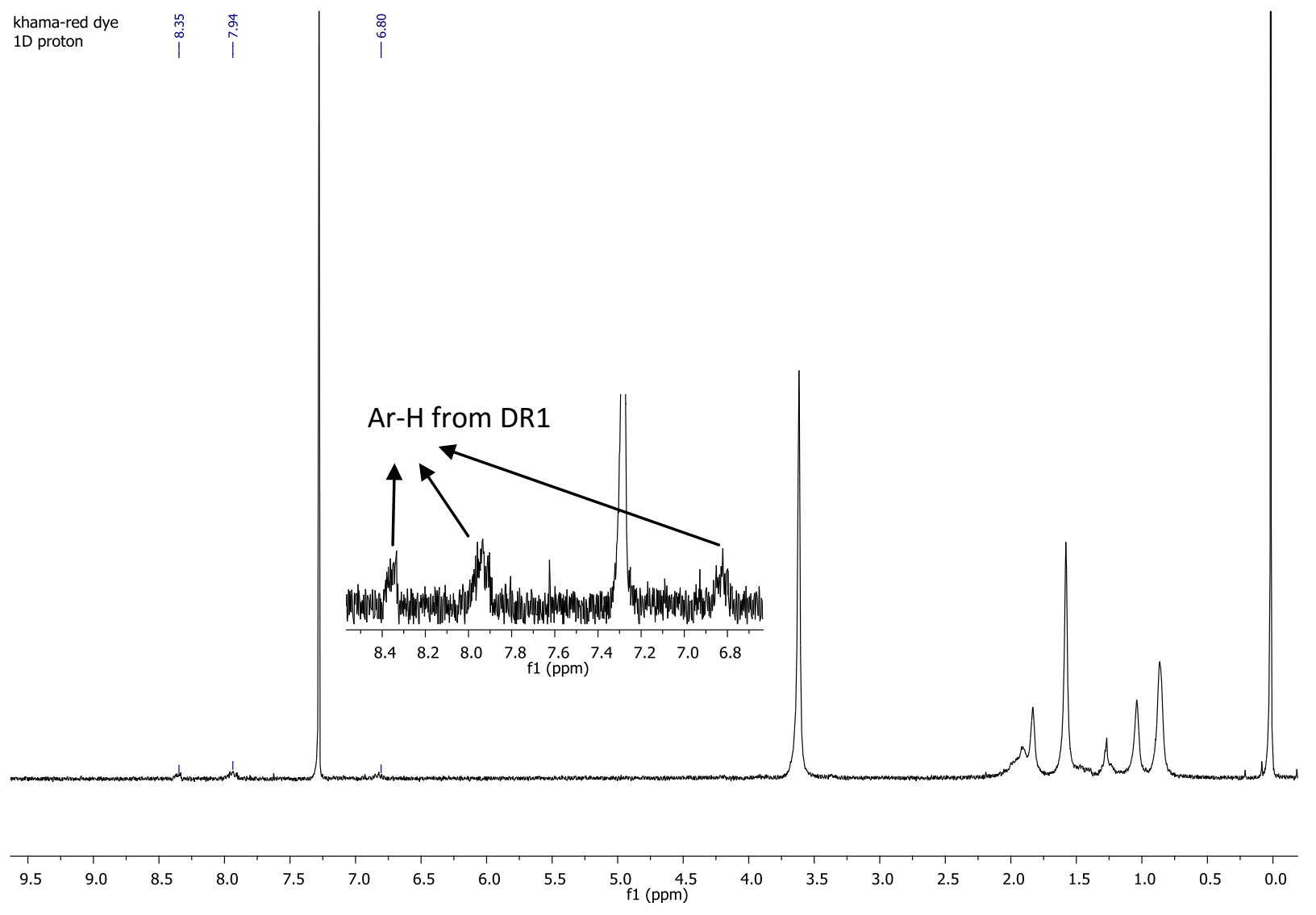

Figure $3.9{ }^{1} \mathrm{H}$ NMR spectrum of polymer P11

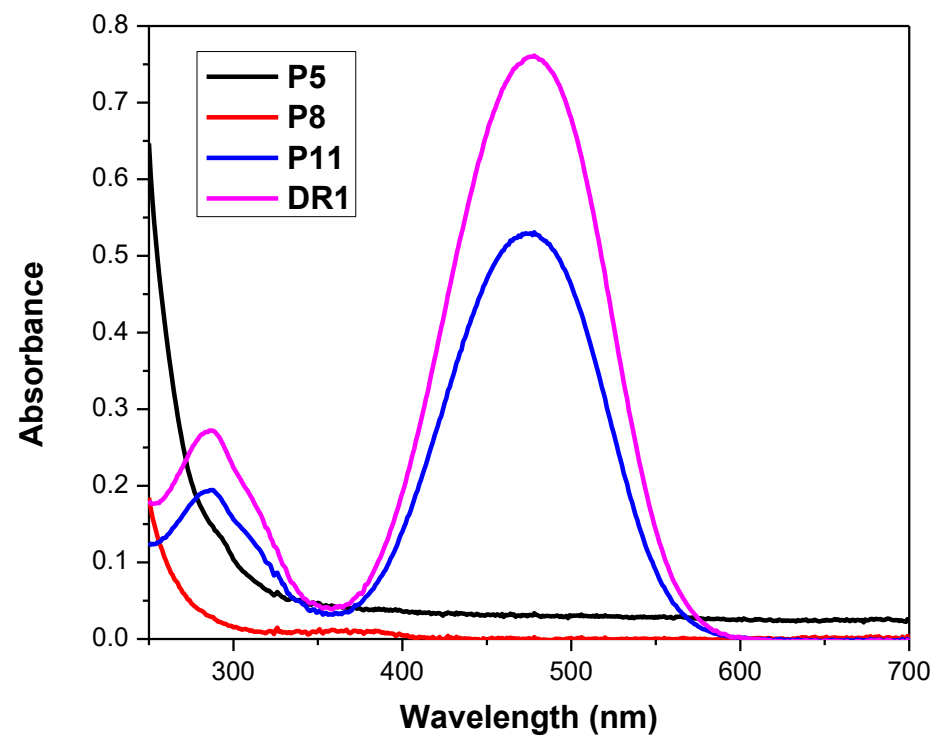

Figure 3.10 Absorption spectra of P5, P8, P11 and DR1 


\subsection{Synthesis and characterizations of MMA-AMAA-HEMA terpolymers}

The NCO-containing polymer could react with any nucleophile for various applications, such as polyurea coatings by reacting with amines. Curing the NCO-containing polymers can be achieved by moisture as well. ${ }^{20}$ Cross-linkable latexes are also based on the NCO-containing copolymers, such as copolymers derived from methyl methacrylate or $n$-butyl acrylate with dimethyl meta-isopropenyl benzyl isocyanate (TMI). ${ }^{24}$

A series of MMA-AMAA-HEMA ternary polymers (P12-P13) were synthesized by free radical polymerization of MMA, AMAA and HEMA with various molar ratios in THF at $60{ }^{\circ} \mathrm{C}$ for 24 hours (Scheme 3.7).

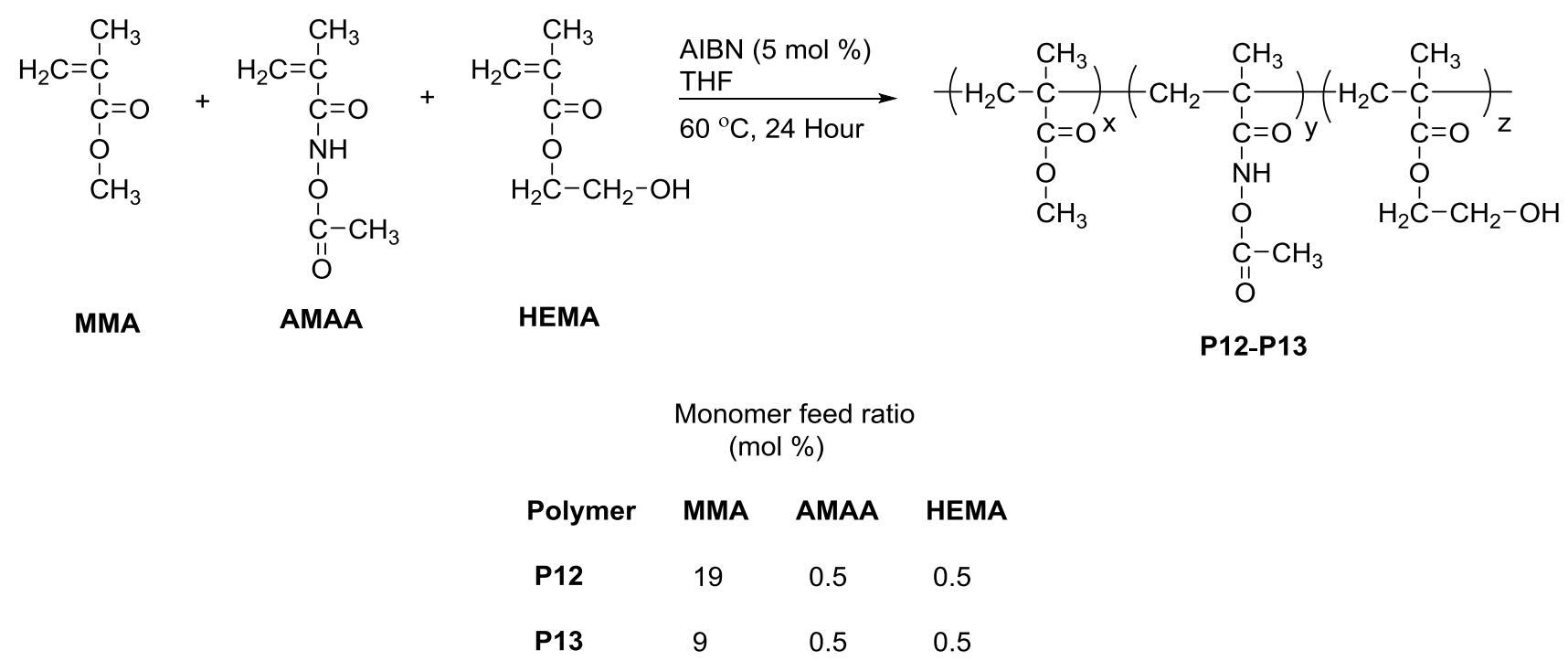

Scheme 3.7 Synthesis of MMA-AMAA-HEMA terpolymers P12-P13

Terpolymers $\mathbf{P 1 2}$ and $\mathbf{P 1 3}$ were fully characterized by the IR, ${ }^{1} \mathrm{H}$ NMR, ${ }^{13} \mathrm{C}$ NMR, TGA, DSC, and GPC. The IR spectra (Figure 3.11a and b) show a peak at $1797 \mathrm{~cm}^{-1}$, which indicates the presence of the AMAA unit in the polymer backbone. The peak intensity at $1797 \mathrm{~cm}^{-1}$ increased with increasing the molar ratio of AMAA. The ${ }^{1} \mathrm{H}$ NMR spectrum (Figure 3.12) shows 
a new peak at 9.43 and $2.21 \mathrm{ppm}$, due to $\mathrm{NH}$ and $\mathrm{O}=\mathrm{C}-\mathrm{CH}_{3}$ of AMAA. In Figure 3.12 the peak intensity at 2.21 increases from $\mathbf{P 1 2}$ to $\mathbf{P 1 3}$, which indicates the molar ratio of AMAA in polymer increases with respect to the ratio in the feed. The ${ }^{1} \mathrm{H}$ NMR spectra (Figure 3.12) also show new peaks at 4.11 and $3.81 \mathrm{ppm}$, which are due to $\mathrm{CH}_{2} \mathrm{COO}-$ and $\mathrm{CH}_{2}-\mathrm{OH}$ of HEMA. Two singlet peaks at $5.73 \mathrm{ppm}$ and $5.49 \mathrm{ppm}$ from methylene protons are shifted to $1.82-1.90 \mathrm{ppm}$ as a broad peak, which indicates the formation of the polymer. The singlet peak at $3.60 \mathrm{ppm}$ is from the $\mathrm{CH}_{3}-\mathrm{O}-\mathrm{C}=\mathrm{O}$ moiety of MMA, indicating the formation of the copolymer with AMAA and HEMA. The ratios of AMAA and HEMA in P12 and P13 are represented in Table 3.3 which are calculated from the ${ }^{1} \mathrm{H}$ NMR spectra of $\mathbf{P 1 2}$ and $\mathbf{P 1 3}$. The actual ratio in the polymer was obtained by comparing with the $\mathrm{CH}_{3}-\mathrm{O}-\mathrm{C}=\mathrm{O}$ peak of MMA with the $\mathrm{O}=\mathrm{C}-\mathrm{CH}_{3}$ of AMAA and HEMA's $\mathrm{O}-\mathrm{CH}_{2}$, respectively. Table 3.3 shows that with increasing the feed in ratio increase the calculated ratio. The ${ }^{13}$ C NMR spectra (Figures S3.19 and S3.23) show two new peaks at 66.87 and $60.58 \mathrm{ppm}$ from $\mathrm{O}_{-} \mathrm{CH}_{2}$ and $\mathrm{CH}_{2} \mathrm{OH}$ of HEMA, confirming the formation of terpolymers $\mathbf{P 1 2}$ and P13. Decomposition temperatures $\left(\mathrm{T}_{\mathrm{d}}\right)$ for $\mathbf{P 1 2}$ and $\mathbf{P 1 3}$ are represented in Table 3.4 and the thermograms are given in Appendix B (Figures S3.20 and S3.24). According to the TGA data, with increasing the molar ratio of AMAA and HEMA in polymers, the onset temperatures for decomposition gradually decrease. The first onset is due to the conversion of AA to NCO. The $\mathrm{T}_{\mathrm{g}}$ of terpolymers $\mathbf{P 1 2}$ and $\mathbf{P 1 3}$ (Figures S3.21 and S3.25) are 128.3 and $127.9{ }^{\circ} \mathrm{C}$, respectively. The DSC traces of $\mathbf{P 1 2}$ and $\mathbf{P 1 3}$ change during the $2^{\text {nd }}$ cycle and show the $T_{g}$ at 123.1 and $122.4{ }^{\circ} \mathrm{C}$, respectively. The polymers $\mathbf{P 1 2}$ and $\mathbf{P 1 3}$ are of high molecular weights (Figures S3.22 and 3.26) with a polydispersity index from 1.69 to 1.61 . 


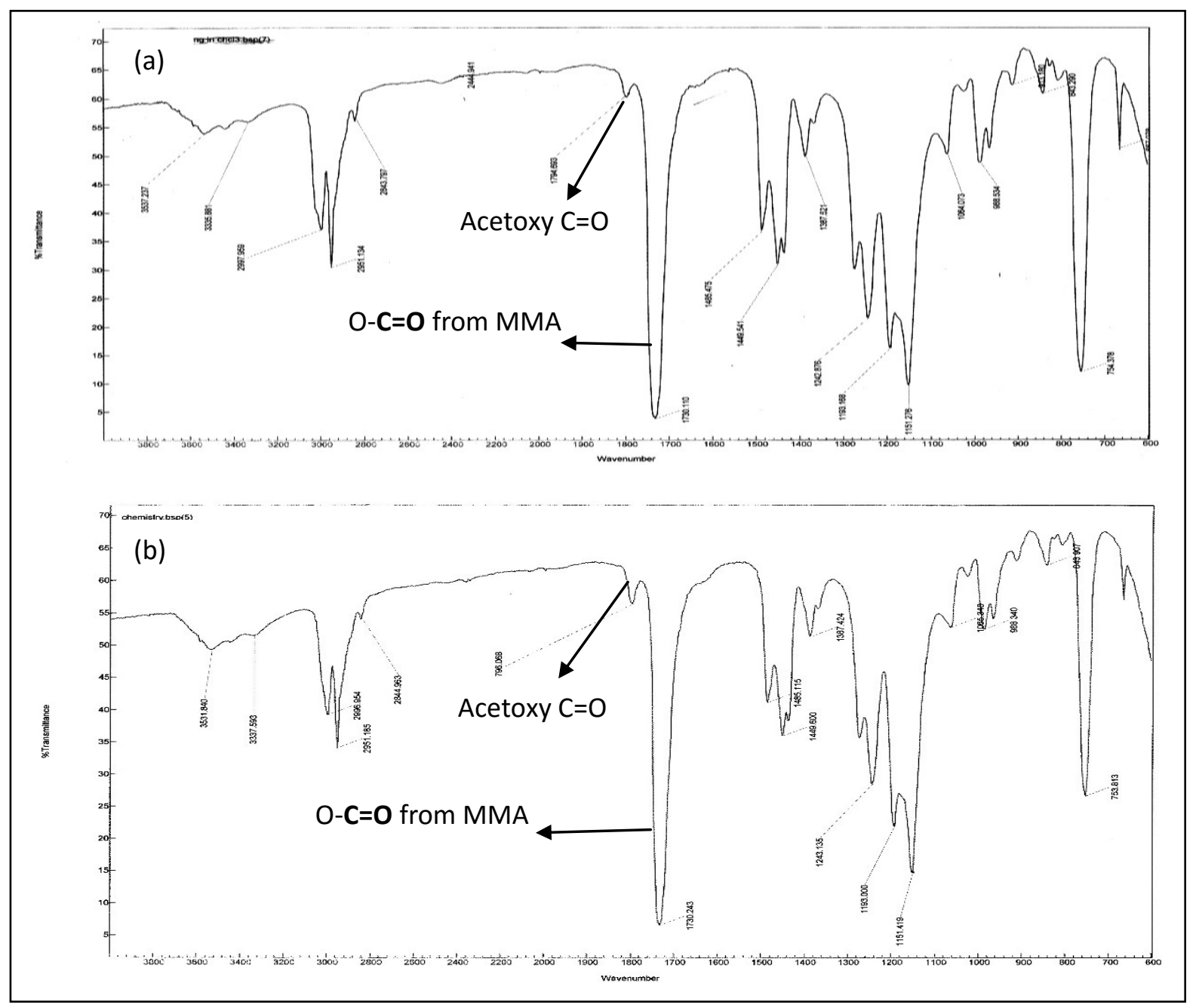

Figure 3.11 IR spectra of terpolymers (a) P12 (containing $2.5 \mathrm{~mol} \%$ of AMAA and $2.5 \mathrm{~mol} \%$ of HEMA), and (b) P13 (containing $5 \mathrm{~mol} \%$ of AMAA and 5 mol \% of HEMA) 


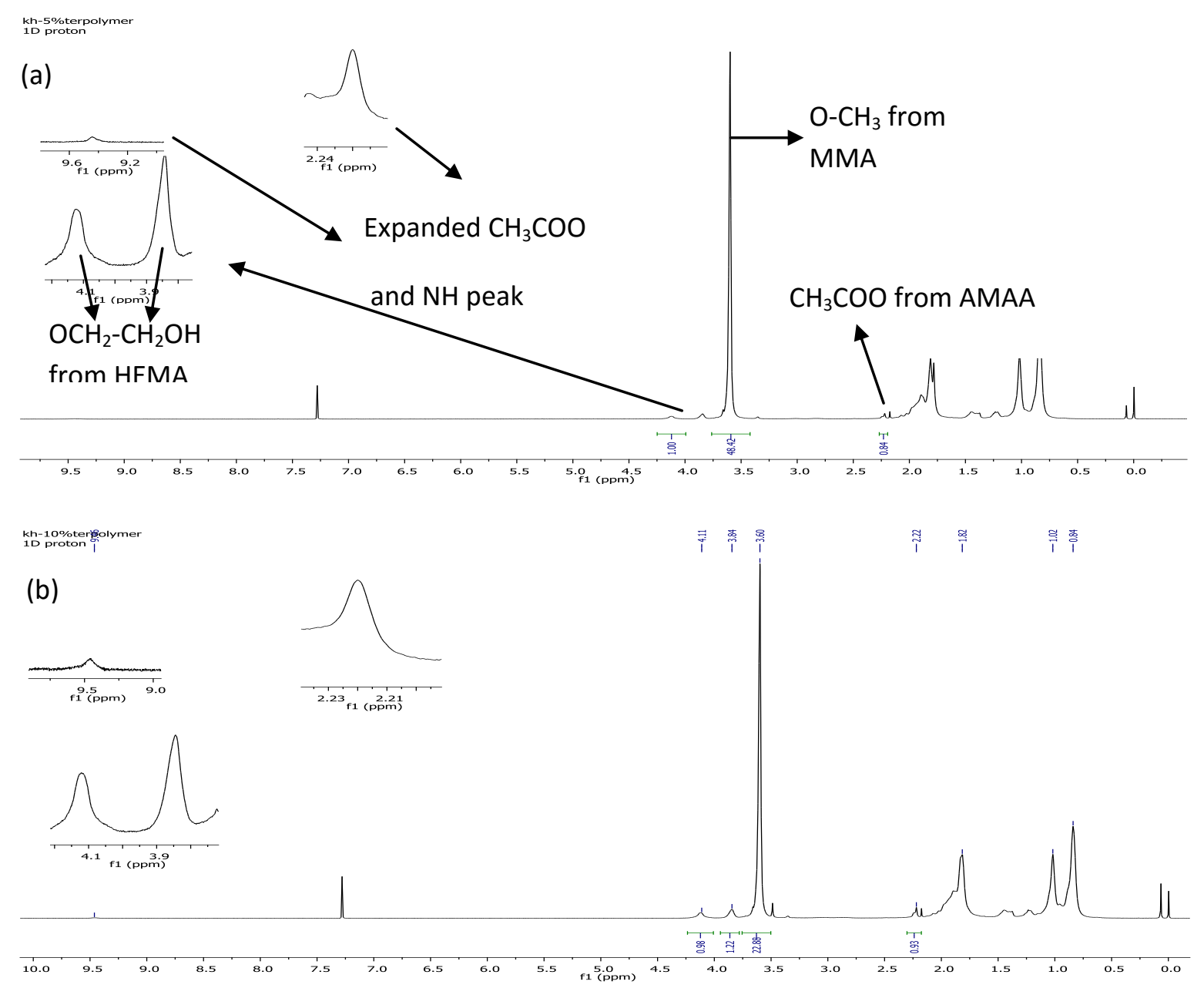

Figure $3.12{ }^{1} \mathrm{H}$ NMR spectra of (a) terpolymers P12 (containing $2.5 \mathrm{~mol} \%$ of AMAA and 2.5 mol \% of HEMA and (b) P13 (containing 5 mol \% of AMAA and 5 mol \% of HEMA)

Table 3.3 The calculated and obtained ratios of monomers in polymers (from ${ }^{1} \mathrm{H}$ NMR spectra)

\begin{tabular}{|c|c|c|c|c|}
\hline Terpolymer & $\begin{array}{c}\text { Feed in ratio of } \\
\text { AMAA (mol \%) }\end{array}$ & Obtained ratio of & Feed in ratio of & Obtained ratio of \\
& AMA (mol \%) & HEMA (mol \%) & HEMA (mol \%) \\
\hline P12 & 2.50 & 1.67 & 2.50 & 1.98 \\
\hline
\end{tabular}


Table 3.4 The onset temperatures for decomposition of terpolymers P12 and P13

\begin{tabular}{|c|c|c|c|}
\hline \multirow{2}{*}{ Terpolymer } & $1^{\text {st }}$ onset & $2^{\text {nd }}$ onset temperature & $3^{\text {rd }}$ onset temperature \\
& temperature $\left({ }^{\circ} \mathrm{C}\right)$ & $\left({ }^{\circ} \mathrm{C}\right)$ & $\left({ }^{\circ} \mathrm{C}\right)$ \\
\hline $\mathbf{P 1 2}$ & 173.96 & 239.82 & 315.64 \\
\hline $\mathbf{P 1 3}$ & 153.11 & 234.14 & 327.96 \\
\hline
\end{tabular}

\subsection{Synthesis and characterizations of polymer P14}

The conversion of copolymer $\mathbf{P 1 2}$ to $\mathbf{P 1 4}$ was studied at $130{ }^{\circ} \mathrm{C}$ and $110{ }^{\circ} \mathrm{C}$ (Scheme 3.8). The DSC and TGA studies indicate that the Lossen reaction of $\mathbf{P 1 2}$ should take place at any temperature between $127^{\circ} \mathrm{C}$ and $153{ }^{\circ} \mathrm{C}$. Under an argon atmosphere, polymer P12 was heated in its powder form at $130{ }^{\circ} \mathrm{C}$ for 1 hour while monitoring by IR spectroscopy.

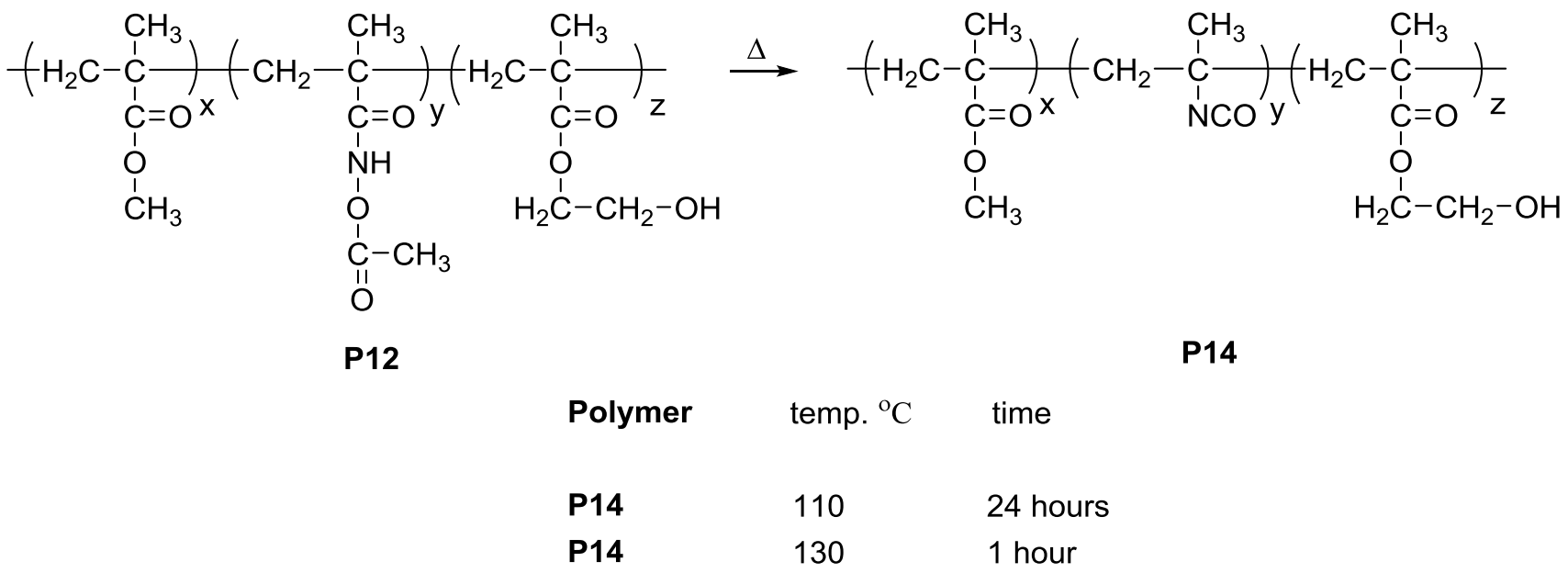

Scheme 3.8 Thermal conversion of terpolymer P12 to P14

The NCO peak appeared after 15 minutes and after 1 hour all the AA groups in P12 were converted to the NCO groups. The IR spectrum (Figure 3.13) of the sample after heating for 1 hour shows a new peak at $2261 \mathrm{~cm}^{-1}$ for NCO moiety and no peak at $1795 \mathrm{~cm}^{-1}$ and $3205 \mathrm{~cm}^{-1}$ 
for the AA group. The observation of the NCO peak in IR spectrum indicates polymer P14 is not crosslinked or does not react with the $\mathrm{OH}$ group of the HEMA part under this condition. The conversion of polymer P12 to P14 at $110{ }^{\circ} \mathrm{C}$ (Figure S3.27) was also successful but required 24 hours to go to completion. Therefore, at $110-130{ }^{\circ} \mathrm{C}$, the AA-containing polymers can be converted to the reactive NCO-containing polymers without crosslinking even with the reactive $\mathrm{OH}$ group presented in the same polymer, which opens a new route to the synthesis of the reactive $\mathrm{NCO}$ - and $\mathrm{OH}$-containing polymers.

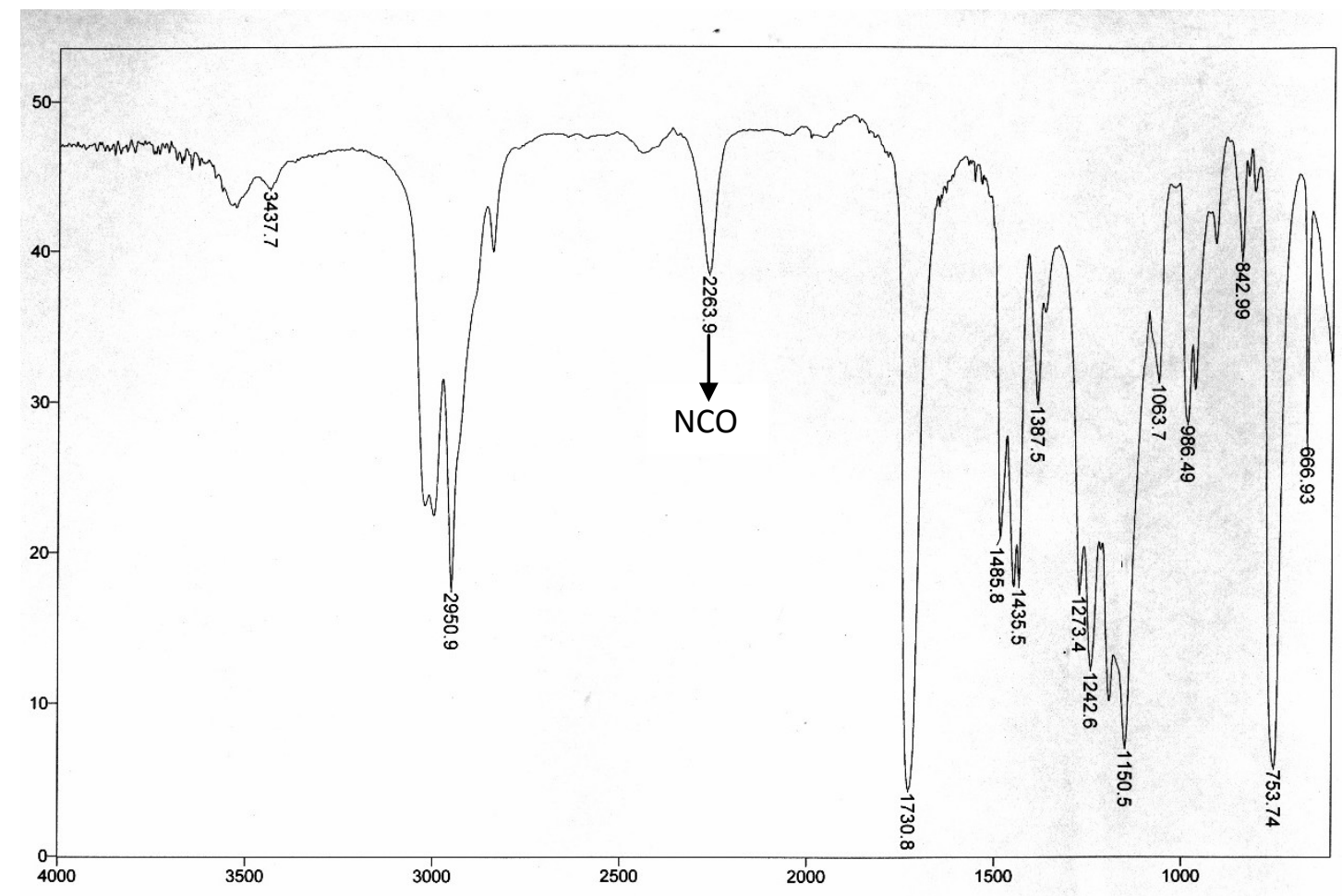

Figure 3.13 Thermal conversions $\left(130^{\circ} \mathrm{C}\right)$ of acetoxyamide group in $\mathbf{P 1 2}$ to $\mathrm{NCO}$ group in $\mathbf{P 1 4}$

\subsection{Study of self-crosslinking NCO- and $\mathrm{OH}-$ containing polymer}

Polymer P14 contains both $\mathrm{NCO}$ and $\mathrm{OH}$ groups and should be reactive by itself even though it could be isolated and characterized. To demonstrate its ability to crosslink, a film of P14 on a glass plate was prepared by casting its solution in chloroform containing $5 \mathrm{~mol} \%$ of 
dibutyltin dilaurate and heated on a pre-heated hot plate at $140{ }^{\circ} \mathrm{C}$ under an argon atmosphere for 10 minutes. The progress of the reaction was monitored by IR spectroscopy. After 10 minutes the glass plate was removed from the hot plate and dipped into chloroform.

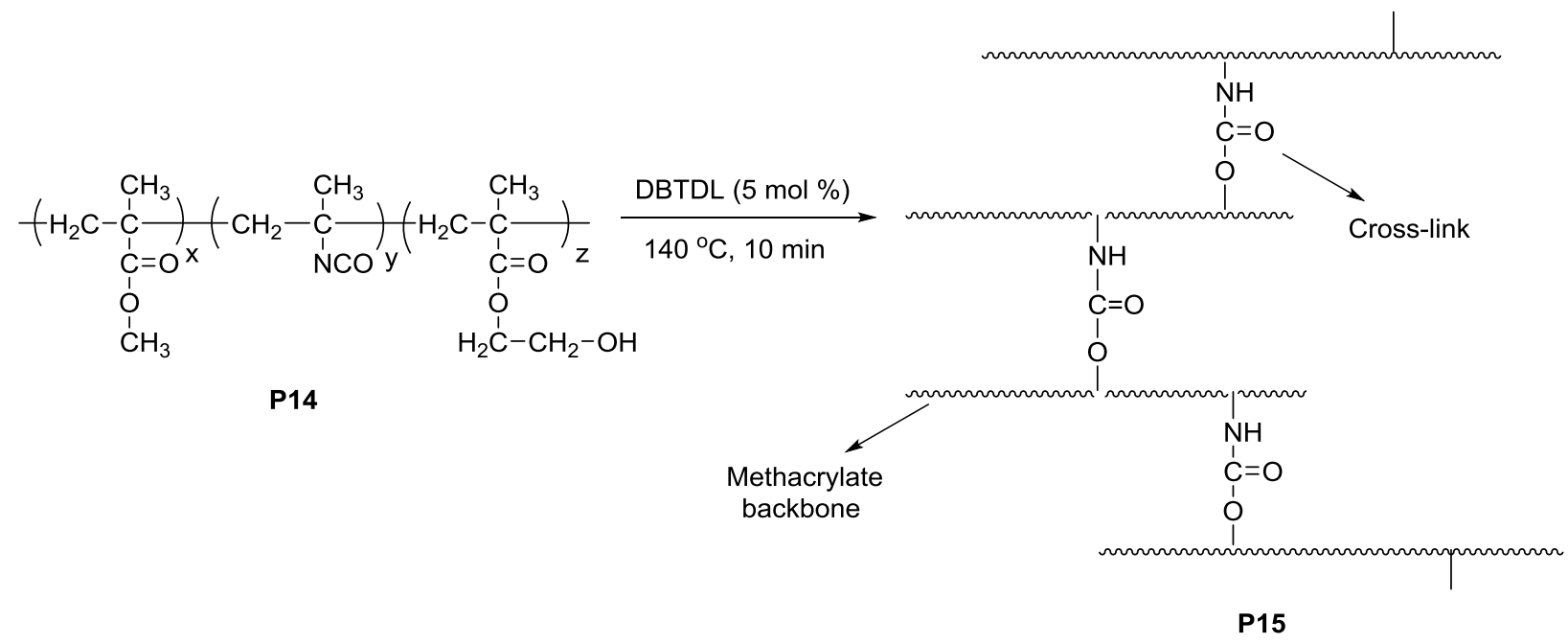

Scheme 3.9 Heating of polymer P14 to form the crosslinked polymer P15

The film was not soluble in chloroform and peeled off from the glass surface (Figure 3.14), indicating the formation of the crosslinked polymer. The IR spectrum of the free-standing film of P15 was taken (Figure 3.15), showing the disappearance of the NCO peak at $2261 \mathrm{~cm}^{-1}$. The urethane $\mathrm{C}=\mathrm{O}$ peak is hard to identify due to the overlap with the ester peak of MMA. The new peak at $1535 \mathrm{~cm}^{-1}$ belongs to is the $\mathrm{N}-\mathrm{H}$ bending and $\mathrm{C}-\mathrm{N}$ stretching, indicating the reaction between $\mathrm{NCO}$ and $\mathrm{OH}$ group in the polymer.

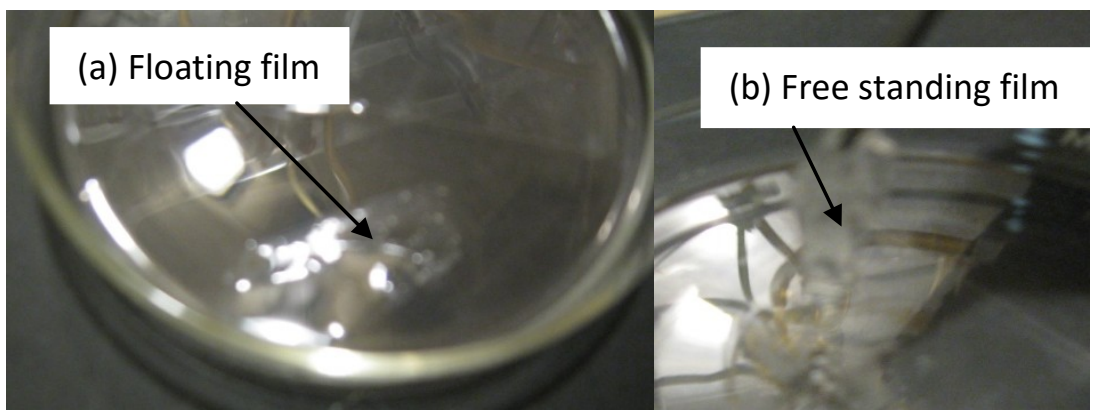


Figure 3.14 The crosslinked film of polymer P15 in chloroform (a) floating and (b) free-standing

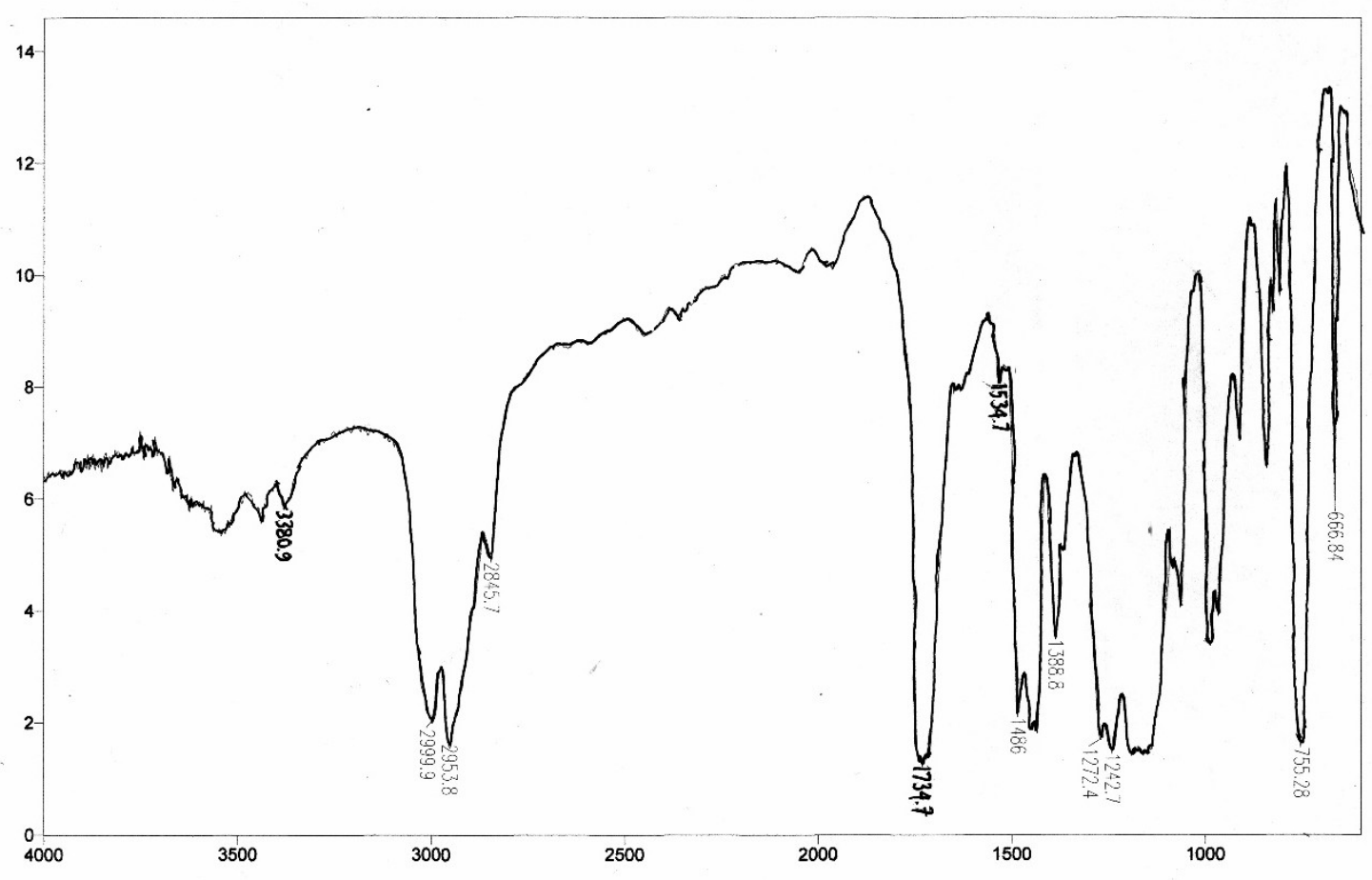

Figure 3.15 IR spectrum of free-standing film of P15 (after heating at $140{ }^{\circ} \mathrm{C}$ )

Another experiment was done to show the adhesion property of the crosslinked polymer P15. A film of polymer P14 containing dibutyltin dilaurate (5 mol \%) was sandwiched between the two glass plates. After heating at $140{ }^{\circ} \mathrm{C}$ for 10 minutes, the glass plates were tightly glued together and could not be pulled apart by force even after soaking in boiling water for 24 hours.

\subsection{Conclusion}

Two series of binary and ternary co-polymers were successfully synthesized by freeradical polymerization and were fully characterized. Successful conversion of these polymers to the corresponding $\mathrm{NCO}$-containing polymers at $110-130{ }^{\circ} \mathrm{C}$ within $1.5 \mathrm{~h}$ were demonstrated and confirmed by IR analysis. The NCO-containing polymers can be generated from the chemically 
inert but thermally sensitive AA-containing polymers and are useful for further reaction with a variety of interesting molecules, as demonstrated by the case of $\mathbf{P 8}$ with a red dye to form a red polymer P11. This work demonstrates a new approach to crosslinkable polymethacrylates with the pre-determined degree of crosslinking, as in the case of polymer P15.

\subsection{Experimental Section}

\section{Materials}

All the reagents were purchased from commercial sources and used as received unless otherwise stated. MMA and HEMA were passed through a column of basic aluminum oxide to remove inhibitor, distilled under argon and stored under an argon atmosphere at $5{ }^{\circ} \mathrm{C}$. Tetrahydrofuran was distilled from sodium and benzophenone prior to use.

\section{N-hydroxymethacrylamide (NHMA)}<smiles>C=C(C)C(=O)NO</smiles>

$\mathrm{NH}_{2} \mathrm{OH} . \mathrm{HCl}(10.4 \mathrm{~g}, 149 \mathrm{mmol})$ was dissolved in $40 \mathrm{~mL}$ of $\mathrm{H}_{2} \mathrm{O}$ in a $250 \mathrm{~mL}$ 3-neck, round-bottomed flask at room temperature. Under an argon atmosphere, $\mathrm{NaOH}(12.0 \mathrm{~g}, 300$ mmol) was dissolved in $20 \mathrm{~mL}$ of $\mathrm{H}_{2} \mathrm{O}$ in a $100 \mathrm{~mL}$, round-bottommed flask and added dropwise to the hydroxylamine solution. Subsequently, $10.0 \mathrm{~g}(99.8 \mathrm{mmol})$ of methyl methacrylate in 40 $\mathrm{mL}$ of methanol was slowly added under vigorous stirring at $0-5{ }^{\circ} \mathrm{C}$. The reaction mixture was stirred at room temperature for $24 \mathrm{~h}$ and then all the solvents were evaporated from the reaction mixture. The reaction mixture was neutralized by $5 \% \mathrm{HCl}(220 \mathrm{~mL})$ and all the liquid was 
evaporated by rotary evaporator. The solid was washed with hot methanol and removed by filtration. The filtrate was evaporated by rotary evaporator and dried in vacuum oven at $30^{\circ} \mathrm{C}$. After drying the pale-yellow liquid obtained initially solidified to a waxy solid in quantitative yield, m.p. $85{ }^{\circ} \mathrm{C}^{19} \mathrm{IR}\left(\mathrm{NaCl}\right.$ Plate) $v_{\max } / \mathrm{cm}^{-1} 3235(\mathrm{NH}$ and $\mathrm{OH}), 1657(\mathrm{O}=\mathrm{CNH}), 1526(\mathrm{C}-\mathrm{N})$. ${ }^{1} \mathrm{H}$ NMR (300 MHz, DMSO-d $\left.{ }_{6}\right) \delta 10.71(\mathrm{~s}, 1 \mathrm{H}), 8.82(\mathrm{~s}, 1 \mathrm{H}), 5.56(\mathrm{~s}, 1 \mathrm{H}), 5.28(\mathrm{~s}, 1 \mathrm{H}), 1.83(\mathrm{~s}$, 3H). ${ }^{13} \mathrm{C}$ NMR (75 MHz, DMSO-d $\left.{ }_{6}\right) 165.75$ (-C=O), 138.08 (=C-), $119.46\left(\mathrm{CH}_{2^{-}}\right), 18.89\left(\mathrm{CH}_{3}-\right)$. MS (EI): Exact mass calculated for $\mathrm{C}_{4} \mathrm{H}_{7} \mathrm{NO}_{2}[\mathrm{M}]^{+}: 101.05001$, found: 101.02001 .

\section{$N$-acetoxymethacrylamide (AMAA)}<smiles>C=C(C)C(=O)NOC(C)=O</smiles>

N-hydroxymethacrylamide $(10.0 \mathrm{~g}, 99.0 \mathrm{mmol})$ and dichloromethane $(150 \mathrm{~mL})$ were added to a $500 \mathrm{~mL}$ round-bottommed flask under an atmosphere of argon. Then $2 \mathrm{M}$ aqueous $\mathrm{NaOH}$ solution ( $3.95 \mathrm{~g}, 98.9 \mathrm{mmol})$ was added dropwise to the suspension and the mixture was stirred for 10 minutes. Following this, acetic anhydride (10.1 g, $98.9 \mathrm{mmol})$ was added dropwise to the mixture and the mixture was stirred for $24 \mathrm{~h}$ at room temperature. Thereafter, water was added to the reaction mixture and then extracted three times with dichloromethane. The organic layer was washed with brine solution and dried over anhydrous $\mathrm{MgSO}_{4}$. The combined dichloromethane layers were concentrated under vacuum and the product is obtained as a dense colorless liquid with $40 \%$ of yield. IR ( $\mathrm{NaCl}$ plate) $v_{\max } / \mathrm{cm}^{-1} 3205(\mathrm{~N}-\mathrm{H}$ stretching), $1795(\mathrm{C}=\mathrm{O}$ stretching for acetoxy), $1672(\mathrm{C}=\mathrm{O}$ stretching for amide), $1575(\mathrm{C}-\mathrm{N}$ stretching and $\mathrm{N}-\mathrm{H}$ bending). ${ }^{1} \mathrm{H}$ NMR (300 MHz, DMSO-d $\left.\mathrm{d}_{6}\right) \delta 11.79$ (s, H), 5.73 (s, 1H), 5.49 (s, 1H), 2.17 (s, 3H), 
$1.87(\mathrm{~s}, 3 \mathrm{H}) .{ }^{13} \mathrm{C}$ NMR (75 MHz, DMSO-d 6$) 169.09(\mathrm{C}(\mathrm{O}) \mathrm{O}), 165.96(\mathrm{C}=\mathrm{O}), 137.00$ (=C-), $121.73\left(\mathrm{CH}_{2}-\right), 18.66$ and $18.60\left(\mathrm{CH}_{3}-\right)$. MS (ESI, DMSO/CH 33$)$ : Exact mass calculated for $\mathrm{C}_{6} \mathrm{H}_{9} \mathrm{NO}_{3}[\mathrm{M}+\mathrm{H}]^{+}: 144.0600$, found: 144.0739 .

\section{$N$-acetoxymethacrylamide-containing polymethacrylates P5-P7}

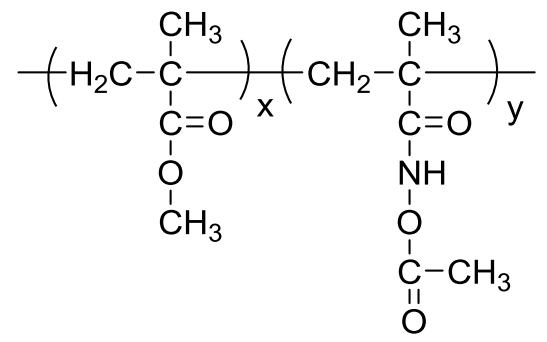

\section{General synthesis by free radical polymerization}

Under an argon atmosphere, methyl methacrylate, $\mathrm{N}$-acetoxymethacrylamide, and THF were added to a flame-dried $25 \mathrm{~mL}$ three-necked round-bottomed flask. After being degassed and purged with argon for $30 \mathrm{~min}, 5 \mathrm{~mol} \%$ of AIBN (relative to all monomers) was added. The reaction mixture was heated at $60{ }^{\circ} \mathrm{C}$ in dark for $24 \mathrm{~h}$ under argon. After cooling, the resulting viscous solution was diluted with Chloroform and precipitated out in methanol (for polymer P5) and in hexane (for polymers $\mathbf{P 6}$ and P7) with vigorous stirring and the target polymers were collected by filtration, which are obtained in a white powdered form and then dried in a vacuum oven at $60{ }^{\circ} \mathrm{C}$ for $8 \mathrm{~h}$.

\section{Polymer P5:}

MMA (4.75 g, $47.5 \mathrm{mmol})$, AMAA $(0.358 \mathrm{~g}, 2.50 \mathrm{mmol})$ and AIBN $(0.410 \mathrm{~g}, 2.50$ mmol). The obtained polymer is white solid ( $70 \%$ yield). IR ( $\mathrm{NaCl}$ plate) $v_{\max } / \mathrm{cm}^{-1} 3329.6(\mathrm{~N}-\mathrm{H}$ 
stretching), $1797(\mathrm{C}=\mathrm{O}$ stretching for acetoxy $), 1731(\mathrm{C}=\mathrm{O}$ stretching for MMA $) .{ }^{1} \mathrm{H}$ NMR $(300$ $\left.\mathrm{MHz}, \mathrm{CDCl}_{3}\right) \delta 9.40$ (NH moiety for acetoxy), $3.61\left(\mathrm{OCH}_{3}\right.$ moiety for MMA), $2.24\left(\mathrm{CH}_{3}\right.$ moiety for acetoxy), $0.97\left(\mathrm{CH}_{2}\right.$ moiety), $0.86\left(\mathrm{CH}_{3}\right.$ moiety). ${ }^{13} \mathrm{C} \mathrm{NMR}\left(75 \mathrm{MHz}, \mathrm{CDCl}_{3}\right) 178.09(\mathrm{C}=\mathrm{O}$ for acetoxy), $177.80\left(\mathrm{C}=\mathrm{O}\right.$ for MMA), $176.97\left(\mathrm{C}=\mathrm{O}\right.$ for acetoxy amide), $54.41\left(\mathrm{O}-\mathrm{CH}_{3}\right.$ for MMA), $51.81\left(\mathrm{CH}_{3}\right.$ for acetoxy), $44.51\left(\mathrm{C}-\mathrm{CH}_{3}\right), 18.69\left(\mathrm{CH}_{2}-\right), 16.44\left(\mathrm{CH}_{3}-\right)$.

\section{Polymer P6:}

MMA (4.50 g, $45.0 \mathrm{mmol})$, AMAA (0.716 g, $5.00 \mathrm{mmol})$ and AIBN (0.410 g, $2.50 \mathrm{mmol})$. The obtained polymer is white solid (70\% yield). IR ( $\mathrm{NaCl}$ plate) $v_{\max } / \mathrm{cm}^{-1} 3329.6(\mathrm{~N}-\mathrm{H}$ stretching), $1797.7\left(\mathrm{C}=\mathrm{O}\right.$ stretching for acetoxy), 1731.1 ( $\mathrm{C}=\mathrm{O}$ stretching for MMA). ${ }^{1} \mathrm{H}$ NMR $(300 \mathrm{MHz}$, $\left.\mathrm{CDCl}_{3}\right) \delta 9.43$ (NH moiety for acetoxy), $3.60\left(\mathrm{OCH}_{3}\right.$ moiety for MMA), $2.25\left(\mathrm{CH}_{3}\right.$ moiety for acetoxy), $1.02\left(\mathrm{CH}_{2}\right.$ moiety), $0.84\left(\mathrm{CH}_{3}\right.$ moiety). ${ }^{13} \mathrm{C} \mathrm{NMR}\left(75 \mathrm{MHz}, \mathrm{CDCl}_{3}\right) 178.13(\mathrm{C}=\mathrm{O}$ for acetoxy), $177.84\left(\mathrm{C}=\mathrm{O}\right.$ for MMA), $176.98(\mathrm{C}=\mathrm{O}$ for acetoxy amide $), 54.34\left(\mathrm{O}-\mathrm{CH}_{3}\right.$ for $\left.\mathrm{MMA}\right)$, $51.86\left(\mathrm{CH}_{3}\right.$ for acetoxy $), 45.63\left(\mathrm{C}-\mathrm{CH}_{3}\right), 18.73\left(\mathrm{CH}_{2}-\right), 16.39\left(\mathrm{CH}_{3}-\right)$

\section{Polymer P7:}

MMA (4.00 g, $40.0 \mathrm{mmol})$, AMAA (1.43 g, $10.0 \mathrm{mmol})$ and AIBN (0.410 g, $2.50 \mathrm{mmol})$. The obtained polymer is white solid ( $87 \%$ yield). IR ( $\mathrm{NaCl}$ plate) $v_{\max } / \mathrm{cm}^{-1} 3329.5$ (N-H stretching), $1797.3\left(\mathrm{C}=\mathrm{O}\right.$ stretching for acetoxy), 1731 ( $\mathrm{C}=\mathrm{O}$ stretching for MMA). ${ }^{1} \mathrm{H}$ NMR (300 MHz, $\left.\mathrm{CDCl}_{3}\right) \delta 9.40$ (NH moiety for acetoxy), $3.61\left(\mathrm{OCH}_{3}\right.$ moiety for MMA), $2.24\left(\mathrm{CH}_{3}\right.$ moiety for acetoxy), $0.97\left(\mathrm{CH}_{2}\right.$ moiety), $0.86\left(\mathrm{CH}_{3}\right.$ moiety). ${ }^{13} \mathrm{C} \mathrm{NMR}\left(75 \mathrm{MHz}, \mathrm{CDCl}_{3}\right): 178.10(\mathrm{C}=\mathrm{O}$ for acetoxy), $177.81\left(\mathrm{C}=\mathrm{O}\right.$ for MMA), $176.92(\mathrm{C}=\mathrm{O}$ for acetoxy amide $), 54.37\left(\mathrm{O}-\mathrm{CH}_{3}\right.$ for MMA), $51.82\left(\mathrm{CH}_{3}\right.$ for acetoxy $), 44.50\left(\mathrm{C}-\mathrm{CH}_{3}\right), 18.69\left(\mathrm{CH}_{2}-\right), 16.38\left(\mathrm{CH}_{3}-\right)$. 


\section{Polymer P11}

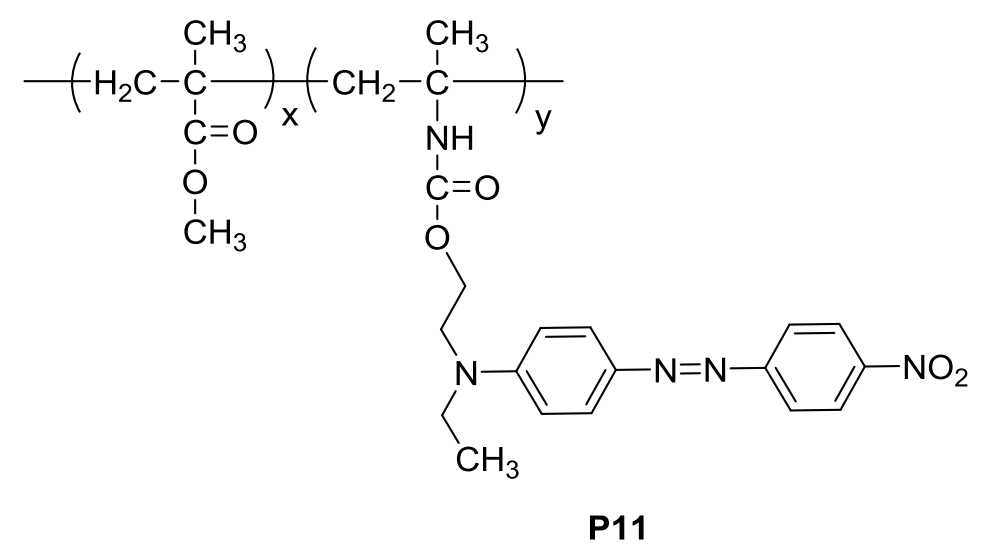

Polymer P11 was synthesized under two different conditions, (a) at $60{ }^{\circ} \mathrm{C}$ (b) at room temperature. The progress of the reaction was monitored by IR spectroscopy.

\section{(a) At $60{ }^{\circ} \mathrm{C}$}

To a flame-dried $25 \mathrm{~mL}$ three-necked round-bottomed flask, $\mathbf{P 8}(0.100 \mathrm{~g}, 1.00 \mathrm{mmol})$ and disperse red $1(0.0157 \mathrm{~g}, 0.0500 \mathrm{mmol})$ in the presence of $5 \mathrm{~mol} \%(0.0006 \mathrm{~g})$ of dibutyltin dilaurate in $4 \mathrm{~mL}$ of chloroform were heated at $60{ }^{\circ} \mathrm{C}$ for $5 \mathrm{~h}$. After cooling, the resulting mixture was evaporated by rotary evaporator and then dissolved in chloroform and precipitated in methanol with vigorous stirring. The polymer was re-precipitated three times in methanol from a solution of chloroform. The target polymer was collected by filtration which is obtained in a reddish powdered form. IR ( $\mathrm{NaCl}$ plate) $v_{\max } / \mathrm{cm}^{-1} 1729.6(\mathrm{C}=\mathrm{O}$ stretching), $1602(\mathrm{C}=\mathrm{C}$ stretching for DR1), 1502 and $1340\left(\mathrm{NO}_{2}\right.$ asymmetric and symmetric stretching for DR1). ${ }^{1} \mathrm{H}$ NMR (300 MHz, $\left.\mathrm{CDCl}_{3}\right) \delta 8.35$ (Ar-H for DR1), 7.95 (Ar-H for DR1) and 6.8 (Ar-H for DR1), 3.61 $\left(\mathrm{OCH}_{3}\right.$ moiety for MMA), $0.85\left(\mathrm{CH}_{2}\right.$ moiety for MMA), $1.03\left(\mathrm{CH}_{3}\right.$ moiety for MMA). 


\section{(b) At room temperature}

The same reaction scale was used except temperature. Now the reaction was continued for 24 hours because 5-8 hours monitoring of the reaction mixture showed still remaining peak of isocyanate. The work-up procedure was similar to heating approach.

\section{Ternary polymers P12-P13}

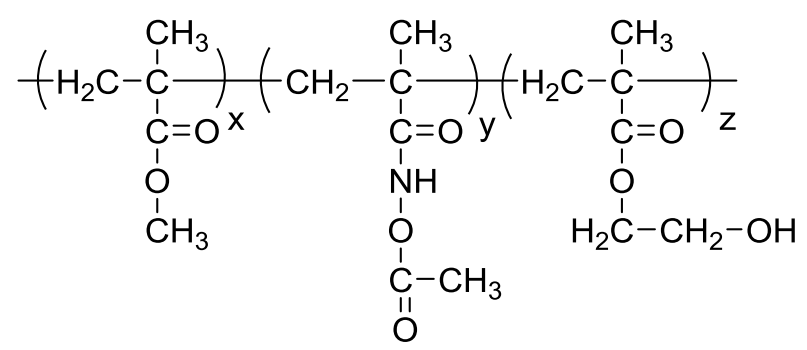

\section{General synthesis by free radical polymerization}

Under an argon atmosphere, in a flame-dried $25 \mathrm{~mL}$ round-bottomed flask, methyl methacrylate, $\mathrm{N}$-acetoxymethacrylamide and 2-hydroxy ethyl methacrylate were dissolved in dry THF. The solution was bubbled with argon for $30 \mathrm{~min}$ and then $5 \mathrm{~mol} \%$ of AIBN was added and the solution was heated to $60^{\circ} \mathrm{C}$ and stirred for $24 \mathrm{~h}$ in a dark to provide a viscous solution. After cooling, the resulted viscous solution was diluted with chloroform and precipitated out in methanol and the target polymer was collected by filtration, which are obtained as a white powdered form and then dried in a vacuum oven at $60{ }^{\circ} \mathrm{C}$ for $8 \mathrm{~h}$.

\section{Polymer P12:}

MMA (2.85 g, 28.5 mmol), AMAA (0.107 g, 0.750 mmol), HEMA (0.0976 g, 0.750 mmol) and AIBN (0.246 g, $1.50 \mathrm{mmol})$. The obtained polymer is light yellowish solid (65\% yield). IR 
( NaCl plate) $v_{\max } / \mathrm{cm}^{-1} 3414.5$ (O-H stretching), 3335.8 (N-H stretching), $1794.6(\mathrm{C}=\mathrm{O}$ stretching for acetoxy), $1730\left(\mathrm{C}=\mathrm{O}\right.$ stretching for MMA). ${ }^{1} \mathrm{H}$ NMR $\left(300 \mathrm{MHz}, \mathrm{DMSO}-\mathrm{d}_{6}\right) \delta 9.45(\mathrm{NH}$ moiety for acetoxy), $4.12\left(\mathrm{OCH}_{2}\right.$ moiety for HEMA), $3.84\left(\mathrm{CH}_{2} \mathrm{O}\right.$ moiety for HEMA), $3.35(\mathrm{OH}$ moiety for HEMA) $2.22\left(\mathrm{CH}_{3}\right.$ moiety for acetoxy). ${ }^{13} \mathrm{C}$ NMR (75 MHz, DMSO-d 6$) 178.41(\mathrm{C}=\mathrm{O}$ for HEMA), $178.12(\mathrm{C}=\mathrm{O}$ for acetoxy), $177.83(\mathrm{C}=\mathrm{O}$ for MMA), $177.00(\mathrm{C}=\mathrm{O}$ for acetoxy amide), 66.91 ( $\mathrm{O}-\mathrm{CH}_{2}$ for HEMA), $60.64\left(\mathrm{CH}_{2} \mathrm{OH}\right.$ for HEMA), 54.21( $\mathrm{O}-\mathrm{CH}_{3}$ for MMA), 51.84 $\left(\mathrm{CH}_{3}\right.$ for acetoxy $), 45.51\left(\mathrm{C}-\mathrm{CH}_{3}\right), 18.69\left(\mathrm{CH}_{2}-\right), 16.42\left(\mathrm{CH}_{3}-\right)$

Polymer P13: MMA (2.70 g, 27.0 mmol), AMAA (0.215 g, 1.50 mmol), HEMA (0.195 g, 1.50 mmol) and AIBN (0.246 g, $1.50 \mathrm{mmol})$. The obtained polymer is light yellowish solid (70 \% yield). IR ( $\mathrm{NaCl}$ plate) $v_{\max } / \mathrm{cm}^{-1} 3414.5$ (O-H stretching), 3337.5 (N-H stretching), $1796.0(\mathrm{C}=\mathrm{O}$ stretching for acetoxy), $1730\left(\mathrm{C}=\mathrm{O}\right.$ stretching for MMA). ${ }^{1} \mathrm{H}$ NMR $\left(300 \mathrm{MHz}, \mathrm{DMSO}-\mathrm{d}_{6}\right) \delta 9.45$ (NH moiety for acetoxy), $4.12\left(\mathrm{OCH}_{2}\right.$ moiety for HEMA), $3.84\left(\mathrm{CH}_{2} \mathrm{O}\right.$ moiety for HEMA), 3.60 (OH moiety for HEMA) $2.22\left(\mathrm{CH}_{3}\right.$ moiety for acetoxy). ${ }^{13} \mathrm{C} \mathrm{NMR}\left(75 \mathrm{MHz}, \mathrm{DMSO}-\mathrm{d}_{6}\right) 178.40$ $(\mathrm{C}=\mathrm{O}$ for HEMA $), 178.11(\mathrm{C}=\mathrm{O}$ for acetoxy $), 177.81(\mathrm{C}=\mathrm{O}$ for MMA $), 176.98(\mathrm{C}=\mathrm{O}$ for acetoxy amide), 66.90 ( $\mathrm{O}-\mathrm{CH}_{2}$ for HEMA), $60.54\left(\mathrm{CH}_{2} \mathrm{OH}\right.$ for HEMA), 54.33 ( $\mathrm{O}-\mathrm{CH}_{3}$ for MMA), 51.84 $\left(\mathrm{CH}_{3}\right.$ for acetoxy), $44.50\left(\mathrm{C}-\mathrm{CH}_{3}\right), 18.69\left(\mathrm{CH}_{2}-\right), 16.39\left(\mathrm{CH}_{3}-\right)$. 


\subsection{References}

1. Itagaki, Y.; Sudo, A.; Endo, T. J. Polym. Sci., Part A: Polym. Chem. 2006, 44, 681.

2. a) Greving, N.; Keul, H.; Millaruelo, M.; Weberskirch, R.; Moeller, M. Euro. Polym. J. 2013, 49, 235. b) Patnaik, B. K.; Zdrahala, R. J. U.S. Patent 6107416, 2000. c) Smith, R. A.; Parker, D. K.; Colvin, H. a.; Weinstein, A. H.; Patterson, D. B.; Cottman, K. S. U.S. patent 0194222 A2, 1986. d) Barr, R. K.; Anzures, E.; Lundy, D. E. U.S. Patent 7364834 B2, 2008. e) Smith, R. A.; Parker, D. K.; Colvin, H. A.; Weinstein, A. H.; Patterson, D. B. U.S. Patent 4694057, 1986.

3. Zhang, X.; Wang, K.; Liu, H.; Gu, Z.; Yang, Y.; Wei, Y.; J. Mater. Chem. C 2015, 3, 1738

4. Moraes, J.; Maschmeyer, T.; Perrier, S. J. Polym. Sci., Part A: Polym. Chem. 2011, 49, 2771.

5. Gody, G.; Rossner, J.; Vana, P.; Maschmeyer, T.; Perrier, S. J. Am. Chem. Soc. 2012, 134, 12596.

6. Greving, N.; Keul, H.; Millaruelo, M.; Weberskirch, R.; Moeller, M. Macromol. Chem. Phys. 2012, 213, 1465.

7. Klinger, D.; Chang, J. Y.; Theato, P. Macromol. Rapid Commun. 2007, 28, 718.

8. Delebecq, E.; Pascault, J. P.; Boutevin, B.; Ganachaud, F. Chem. Rev. 2013, 113, 80.

9. Risangud, N.; Congdon, T. R.; Keddie, D. J.; Wilson, P.; Kempe, K.; Haddleton, D. M. J. Polym. Sci., Part A: Polym. Chem. 2016, 54, 2698.

10. Dexter, R. W.; Saxon, R.; Fiori, D. E. Polym. Mat. Sci. Eng. 1985, 53, 534.

11. a) Brauer, G. M.; Lee, C. H. J. Biomed. Mater. Res. 1989, 23, 295. b) Parker, D. K.; Colvin, H.A.; Weinstein, A. H.; Chen, S.-L. Rubber Chem. Technol. 1990, 63, 582. c). Ekman, K.B.; Nakman, J. H. Polym. Prepr. 1994, 34, 483.

12. Kiguchi, T.; Aota, H.; Matsumoto, A. Macromolecules 2004, 37, 8249. 
13. Kiguchi, T.; Aota, H.; Matsumoto, A. J. Polym. Sci., Part A: Polym. Chem. 2003, 41, 3243.

14. Seo, S. H.; Chang, J. Y. Polym. Prepr. 2002, 43, 1168.

15. Singh, R. P.; Patwa, A. N.; Desai, S. M.; Pandey, J. K.; Solanky, S. S.; Prasad, A. V. J. Appl.Polym. Sci. 2003, 90, 1126.

16. Brandenberger, H. Helv. Chim. Acta 1957, 40, 61.

17. Iwakura, Y.; Uno, K.; Nakabayashi, N.; Kojima, T. Bull. Chem. Soc. Jpn. 1968, 41, 186.

18. Veyries, M.-L.; Faurisson, F.; Joly-Guillou, M.-L.; Rouveix, B. Antimicrob. Agents Chemother. 2000, 44, 1093.

19. a) Basheer, R. A.; Jodeh, S.; Hopkins, A. R. Mat. Res. Innoval, 2001, 4, 144. b) de Lima, J. R.; Schreiner, C.; Hummelgen, I. A.; Fornari Jr, C. C. M.; Ferreira, C. A.; Nart, F. C. J. App. Phys. 1998, 84, 1445. c) Wang, Y.; Jing, X. Mater. Sci. Eng. B 2007, 138, 95. d) Jin, S.; Tiefel, T. H.; Wolfe, R.; Sherwood Jr. R. C.; Mottine, J. J. Science, 1992, 255, 446. e) Vijayaraghavan, G. V.; Ahamed, M. B. Spectrochimica Acta Part A: Molecul. Biomole. Spectro. 2016, 158, 43.

20. a) Kern, W.; Schultz, R. C. Angew Chem. 1957, 69, 153. b) Narita, M. Bull. Chem. Soc. Jpn. 1972, 45, 3149.

21. Ni, H.; Skaja, A. D.; Sailer, R. A.; Soucek, M. D. Macromol. Chem. Phys. 2000, 201, 722.

22. (a) Gogai, R.; Alam, M. S.; Khandal, R. K. Int. J. Basic Appl. Sci. 2014, 3, 118. (b) Beck, K. R.; Korsmeyer, R.; Kunz, R. J. J. Chem. Edu. 1984, 61, 668.

23. Biswas, N.; Umapathy, S. J. Phys. Chem. A 2000, 104, 2734.

24. Lovell, P. A.; Yoon, J. J. Macromol. Sci. Part B: Phys. 2005, 44, 1041. 


\section{Chapter 4 Detection of Ultralow Trace Amounts of Isocyanates in Air Using Fluorescent Conjugated Polymer}

\subsection{Introduction}

\subsubsection{Overview of conjugated polymers}

Conjugated polymers have received much attention as signal-transducing elements for sensory applications due to their signal amplification properties and the versatility in their molecular design. ${ }^{1,2}$ Fluorescent conjugated polymers have been employed extensively as sensing materials almost three decades. ${ }^{3-5}$ This method based on upon changes in fluorescence intensity, energy transfer, electron transfer, wavelength (excitation and emission), and lifetime. ${ }^{6}$ Fluorescence is a leading signal transduction method for the formation of the chemosensory device. One of the main features that have accelerated the use of fluorescent conjugated polymers as sensors relate to their ability to produce the signal gain in response to an interaction with target analytes. The $\pi$ electrons along the polymer chain are available for delocalization all over the backbone. Excitons in conjugated polymers are mobile and can migrate throughout the polymer backbone; this migration increases the frequency of interaction with the bound quencher, as a result, the sensitivity becomes higher.

To understand this chemistry, studies were done in parallel with the small molecule receptor and polymeric analogues of different molecular weights. The result shows that both displayed quenching towards the analyte by forming a complex between the receptor and analyte. The polymer has a greatly enhanced sensitivity over the small molecular receptor. Further investigations have also indicated that the signal amplification increases with molecular weight. Once the molecular weight exceeds the average diffusion length of the excitation (ca. Mn $=100000)$, the effect is independent of molecular weight. ${ }^{3}$ 
In this part of the thesis two main properties of the analyte were considered for detection; 1) electron deficiency 2) volatility. The isocyanato group is electron deficient due to the electron withdrawing nature by electronegative atom oxygen and nitrogen from the carbon atom. The volatility (high vapor pressure) of isocyanates allows for detection in air by electron-rich conjugated polymers. Herein we demonstrate the detection of 8 different types of commercially available aromatic and aliphatic isocyanates. Selectivity of fluorescent conjugated polymer sensors can be improved by (a) filtering the analytes and then introduced to the sensor, (b) adsorbing the analytes on sensor and then followed desorption by heating and (c) creating a fluorescence character map based on analytes.

\subsubsection{Contribution}

The following is a full version of the article "Direct detection of ultralow trace amounts of isocyanates in air using a fluorescent conjugated polymer" published on November $8^{\text {th }}, 2013$ in Chemical Communications and reprinted with permission from The Royal Society of Chemistry Copyright Clarence Centre (see Appendix C). This includes detailed supporting information with optimization data and tables as well as additional unpublished data, provided therein. The synthesis of TPE (tetraphenylethylene) monomer and polymerization were carried out by another student, Sukanta Kumar Saha. Some difficulties during the synthesis of pentiptycene monomer and film preparation were solved by Dr. Jian Ping Gao. Manuscript writing, synthesis of monomer pentiptycene and rest of the experimental work including film preparation and sensitivity of the polymer towards 8 different types of isocyanates as well as the related calculations were carried out by me.

The link to the article: http://pubs.rsc.org/en/content/articlehtml/2014/cc/c3cc47934j 


\subsubsection{Abstract}

A fluorescence sensory polymer containing the pentiptycene and tetraphenylethylene units linked by acetylene was synthesized for direct detection of isocyanates in air. Eight industrially available aliphatic and aromatic isocyanates were tested. The polymer film shows a rapid fluorescence quenching response to any type of isocyanates with a ppt level of detection limit, which is much lower than the permissible exposure limit of $5 \mathrm{ppb}$. 


\subsubsection{References}

1. Thomas, S.W.; Joly, G. D.; Swager, T. M. Chem. Rev. 2007, 107, 1339.

2. Duan, X.; L. Liu, L.; Feng, F.; Wang, S. Acc. Chem. Res. 2009, 43, 260.

3. Zhou, Q.; Swager, T. M. J. Am. Chem. Soc. 1995, 117, 7017.

4. Cumming, C. J.; Aker, C.; Fisher, M.; Fox, M.; la Grone, M. J.; Reust, D.; Rockley, M. G.; Swager, T. M.; Towers, E.; Williams, V. IEEE Trans. Geosci. Rem. Sens. 2001, 39, 1119.

5. Toal S. J.; Trogler, W. C. J. Mater. Chem. 2006, 16, 2871.

6. McQuade, D. T.; Pullen, A. E.; Swager, T. M. Chem. Rev. 2000, 100, 2537.

7. Chiang, C. K.; Fincher, C. R.; Park, Y. W.; Heeger, A. J.; Shirakawa, H.; Louis, E. J.; Gau, S. C.; MacDiarmid, A. G. Phys. Rev. Lett. 1977, 39, 1098. 


\subsection{Article}

Isocyanates are highly reactive and low molecular weight compounds containing the isocyanato group $(-\mathrm{N}=\mathrm{C}=\mathrm{O})$. There are three common types of isocyanates available: mono-, di/tri- and polyisocyanates and among them polyisocyanates are not volatile at room temperature. ${ }^{1-}$

${ }^{4}$ Isocyanates are widely used in large quantity in many industrial sectors, such as automobile, construction, and sports. The most commonly used diisocyanates are 4,4-methylenedisocyanate (MDI), 2,4-toluene diisocyanate (TDI), hexamethylene diisocyanate (HDI), isophorone diisocyanate (IPDI), 1,5-naphthalene diisocyanate (NDI), and $p$-phenylene diisocyanate (PPDI). ${ }^{5-}$ ${ }^{8}$ Exposure to even a small amount of isocyanates can cause irritation to the eyes and skin and also decreased lung function when inhaled in the form of gas, steam, dust or aerosol. ${ }^{9,10}$ The most common adverse health impact associated with isocyanate exposure is asthma due to sensitization. ${ }^{11}$ In most countries, the permissible exposure limit (PEL) for most isocyanate monomers is $5 \mathrm{ppb}$ in air. ${ }^{12}$ This implies that the detection of isocyanate compounds in a workplace is highly required to maintain a safe working environment and protect the workers from adverse health effects. The most commonly used analytical methods for the detection of isocyanates in air are colorimetric methods, ${ }^{13}$ absorption spectrophotometry, ${ }^{14-17}$ liquid chromatography with fluorescence detection using N-methyl-1-naphthalenemethylamine as a fluorescence-activating reagent, ${ }^{18}$ capillary zone electrophoresis as an alternative to HPLC, ${ }^{19}$ and mass spectrometry. ${ }^{20}$ A recently developed and improved method to detect isocyanates is PTRMS (proton transfer reaction-mass spectrometry). ${ }^{21}$ Nearly all the analytical techniques require chemical derivatizations and separation. Most methods are limited to a few isocyanates with the unknown detection limits, except for the one by alkaline adduct coordination ion spray tandem mass spectrometry for determining specifically three isocyanates (HDI, 2,4-TDI, and 2,6-TDI) at ppt levels in air. ${ }^{20 a}$ 
Highly fluorescent conjugated polymers are used as molecular sensors for direct detection of various chemicals, such as nitroaromatic explosives. ${ }^{22,23}$ Conjugated polymers are able to transfer their energy from the excited state to the electron deficient analytes and cause fluorescence turn-off. In comparison with nitro-compounds, isocyanates are also electron deficient and have sufficient vapor pressure at room temperature. Accordingly, isocyanates should be detectable, like nitro-compounds, by a fluorescence quenching mechanism using a conjugated polymer.

For direct detection of isocyanates in air by the fluorescence method, we designed a conjugated polymer containing the pentiptycene and tetraphenylethylene (TPE) units that are linked by an acetylene bond, namely polymer P1 (Scheme 1). Pentiptycene is selected as one of the key structural components of polymers, since it is readily available by simple synthesis ${ }^{24,25}$ and is proven to be useful in polymers for fluorescence detection of nitro explosives. ${ }^{26}$ Pentiptycene has a three dimensional rigid structure, which prevents $\pi$-stacking or excimer formation in the excited state in the solid film. ${ }^{26,27}$ TPE is a known luminophore and in particular is highly emissive in the aggregated state or has the aggregation-induced emission (AIE) effect. ${ }^{28}$ In addition to some unique applications, ${ }^{29-34}$ the polymers with the AIE property have been used as chemosensors. ${ }^{35-37}$ Therefore, polymer $\mathbf{P 1}$ is expected to be highly emissive as the solid film, due to the presence of the aggregated TPE moiety. The aggregated TPE and the bulky pentiptycene moieties create the molecular cavities in polymer film, which allow for the analytes to enter and get trapped readily. Thus, these molecular features and characteristics are deemed to make polymer P1 suitable for sensing various isocyanates.

Herein we represent the synthesis and properties of a sensory polymer with a high sensitivity towards isocyanates. Thin films of polymer $\mathbf{P 1}$ are able to detect various aliphatic, 
aromatic, mono- and diisocyanates at the permissible exposure limit at room temperature. In our work, we selected pentiptycene diacetylene (1) and 1,2-bis(4-bromophenyl)-1,2-diphenylethene (2) as monomers. Monomers $\mathbf{1}$ and $\mathbf{2}$ were synthesized according to the standard procedure from the literature. ${ }^{38,39}$ The synthetic route to the target sensory polymer $\mathbf{P 1}$ is shown in Scheme 1. The Sonogashira cross-coupling reaction of compound $\mathbf{1}$ with compound $\mathbf{2}$ affords polymer $\mathbf{P 1}$ in $71 \%$ yield. ${ }^{40}$
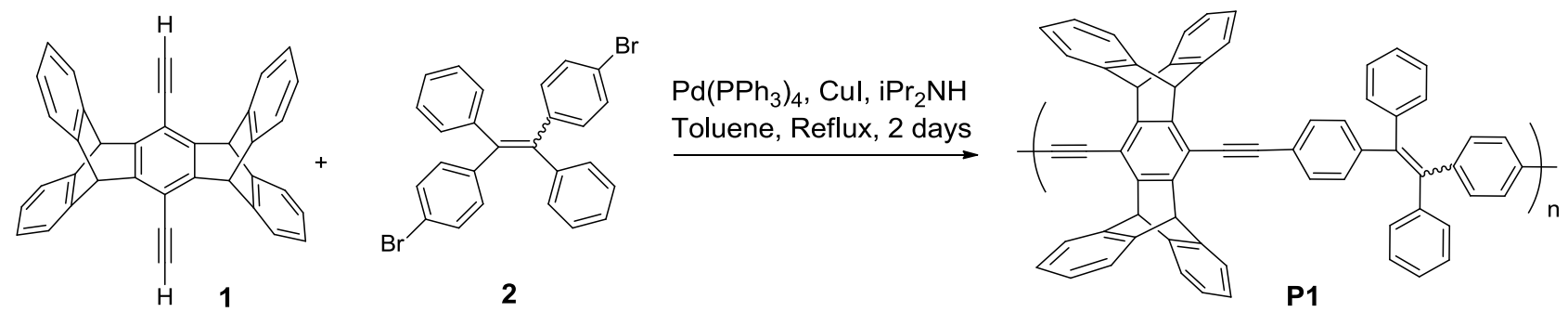

Scheme 1. Synthesis of polymer $\mathbf{P 1}$ by Sonogashira cross-coupling reaction.

Polymer P1 is highly soluble in THF and TCE and partially soluble in $\mathrm{CHCl}_{3}, \mathrm{CH}_{2} \mathrm{Cl}_{2}$, and DMF. Polymer P1 has an inherent viscosity of $0.20 \mathrm{dL} \mathrm{g}^{-1}$ in TCE at $30{ }^{\circ} \mathrm{C}$ and forms a uniform and smooth film on glass surface by casting or spin coating indicating reasonably high molecular weight. The ${ }^{1}$ H-NMR spectrum (Fig. S1) of P1 displays the peaks for aromatic protons at 6.2 to $7.2 \mathrm{ppm}$ but no peak at $3.71 \mathrm{ppm}$ for the acetylenic proton. The band at 2203 $\mathrm{cm}^{-1}$ for the $\mathrm{C} \equiv \mathrm{C}$ bond in the IR spectrum (Fig. S2) also confirms the polymer formation. Further evidence comes from the ${ }^{13} \mathrm{C}-\mathrm{NMR}$ data (Fig. S3), which clearly reveals that the acetylene carbons at 78.03 and 84.84 ppm for monomer 1 shift to 124.88 and $123.52 \mathrm{ppm}$ for polymer $\mathbf{P 1}$. Fig. S6 displays the normalized absorption and emission spectra of polymer P1 in solution and in film. In solution, polymer $\mathbf{P 1}$ shows a maximum absorption at $363 \mathrm{~nm}$ whereas monomers $\mathbf{1}$ and 2 have the absorption maxima at $332 \mathrm{~nm}$ and $317 \mathrm{~nm}$, respectively. Polymer P1 emits at $488 \mathrm{~nm}$, whereas monomers 1 and 2 show the emission maxima at $356 \mathrm{~nm}$ and $437 \mathrm{~nm}$, respectively. A red shift in absorption and emission spectra of polymer $\mathbf{P} \mathbf{1}$ relative to monomers $\mathbf{1}$ and $\mathbf{2}$ resulted 
from extension of $\pi$-conjugation in the polymer backbone. The film of polymer P1 absorbs broadly in comparison with the one in solution and emits at $504 \mathrm{~nm}$ with a quantum yield of 17\%. From SEM images (Figure S14C), the average pore size of the film surface is estimated to be $97 \mathrm{~nm}$.

In sensing experiments, polymer films were exposed to the vapor of isocyanates at room temperature and the fluorescence spectra were recorded immediately after isocyanate exposure for a specific period. Fluorescence Quenching Response (FQR) can be defined as the percent ratio of fluorescence intensity decrease upon exposure and initial fluorescence intensity. Fig. 1(a) displays the gradual changes in the fluorescence intensity upon exposure to MDI vapor over a period of $300 \mathrm{~s}$. After $60 \mathrm{~s}$ of exposure to MDI, the fluorescence intensity of $\mathbf{P 1}$ dropped by $74 \%$ or $74 \% \mathrm{FQR}$, and continued to drop by $84 \%$ at 300 s. Figure 1(b) shows the changes in the fluorescence intensity in the presence of IPDI vapor. The FQR was $52 \%$ at $60 \mathrm{~s}$ and $67 \%$ at 300 s. By comparing the FQR for MDI and IPDI, it can be concluded that polymer $\mathbf{P 1}$ is sensitive towards both aromatic and aliphatic isocyanates.
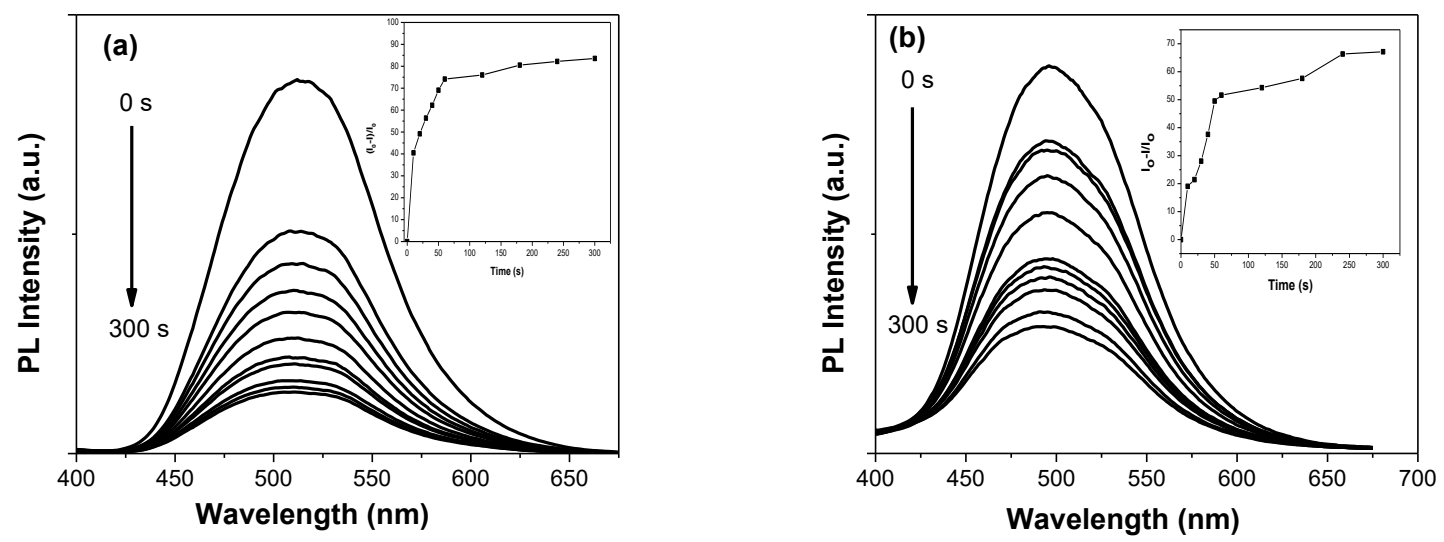

Figure 1. The time- dependent fluorescence intensity of P1 film (7 nm) upon exposure to (a) MDI (b) IPDI vapor (room temperature) from $0 \mathrm{~s}$ to $300 \mathrm{~s}$. 
In the isocyanato group, the carbon atom is partially positively charged, as it is doubly bonded to more electronegative $\mathrm{N}$ and $\mathrm{O}$ atoms. These electronegative atoms pull electrons away from the carbon atom, making the isocyanato group quite electron deficient. The high reduction potential of the isocyanato group allows for an easy electron transfer and thus effective fluorescence quenching. The pentiptycene and TPE moieties create a large number of cavities or free volume in polymer film, which permits a high diffusion rate for isocyanate molecules to enter the polymer film, thus leading to a high FQR within a short period of exposure. Experimentally, the fluorescence signals decrease noticeably within only $10 \mathrm{~s}$ of isocyanate exposure (Table 1) and become constant after $5 \mathrm{~min}$, indicating that the concentration of analyte molecules in the polymer film with a thickness of $4-30 \mathrm{~nm}$ has reached the equilibrium at room temperature.

To further prove the sensitivity of polymer P1 towards isocyanates, 8 different isocyanates, including phenyl isocyanate (PI), 4-methoxyphenyl isocyanate (MeOPI), 4nitrophenyl isocyanate $\left(\mathrm{NO}_{2} \mathrm{PI}\right)$, MDI, TDI, HDI, IPDI, PPDI, were tested. The FQR data at given time are summarized in Table 1 and the other spectroscopic measurements are given in the supporting information.

At $60 \mathrm{~s}$ of exposure, the order of the $\mathrm{FQR}$ is $\mathrm{MDI}>\mathrm{PPDI}>\mathrm{PI}>\mathrm{TDI}>\mathrm{HDI}>\mathrm{NO}_{2} \mathrm{PI}>$ MeOPI > IPDI. The experimental results indicate that polymer P1 can maintain a high sensitivity towards aliphatic and aromatic isocyanates. The detection sensitivity or FQR relates to the vapor pressure of the isocyanate. For aromatic mono-isocyanates, polymer P1 shows a higher sensitivity towards phenyl isocyanate, due to the higher vapor pressure of PI (1.4 mmHg, Table $\mathrm{S} 1)$ than that of MeOPI $(0.107 \mathrm{mmHg})$ or $\mathrm{NO}_{2} \mathrm{PI}\left(6.84 \times 10^{-3} \mathrm{mmHg}\right)$. The detection sensitivity is also related to the nature or reduction potential of isocyanates. Polymer P1 shows a higher 
sensitivity towards aromatic diisocyanates than aliphatic diisocyanates, because the former is more electron deficient than the latter, even though MDI has a much lower vapor pressure $(4.5 \mathrm{x}$ $\left.10^{-6} \mathrm{mmHg}\right)$ than HDI $\left(5.26 \times 10^{-3} \mathrm{mmHg}\right)$ or IPDI $(3.0 \times 10-4 \mathrm{mmHg})$. For the same reason, PPDI has a vapor pressure less than $6.0 \times 10^{-3} \mathrm{mmHg}$ but due to its highly electron-deficient nature causes the highest FQR for polymer P1. Since MDI has the lowest vapor pressure or vapor concentration of $0.062 \mathrm{ng} \mathrm{mL}^{-1}$ among all the eight isocyanates tested, the detection limit of polymer P1 should be at a ppt level (i.e., $0.06 \mathrm{ppt}$ ) and is much lower than the permissible exposure limit (PEL) of 5 ppb.

Table 1. Fluorescence quenching response (\%) at a given time for various isocyanates using isocyanates using polymer $\mathbf{P 1}^{a}$

\begin{tabular}{llllllllll}
\hline Time, $\mathrm{s}$ & 10 & 20 & 30 & 40 & 50 & 60 & 120 & 180 & 240 \\
\hline PPDI & 23 & 31 & 48 & 54 & 63 & 71 & 75 & 78 & 81 \\
MDI & 41 & 49 & 56 & 62 & 69 & 74 & 76 & 81 & 82 \\
TDI & 22 & 30 & 42 & 52 & 60 & 63 & 67 & 70 & 75 \\
PI & 21 & 30 & 41 & 52 & 60 & 66 & 70 & 73 & 77 \\
$\mathrm{NO}_{2} \mathrm{PI}$ & 14 & 18 & 32 & 40 & 49 & 61 & 66 & 69 & 72 \\
MeOPI & 22 & 31 & 41 & 45 & 54 & 59 & 61 & 65 & 66 \\
$\mathrm{HDI}$ & 24 & 26 & 40 & 45 & 53 & 62 & 67 & 71 & 73 \\
IPDI & 19 & 21 & 28 & 38 & 50 & 52 & 54 & 58 & 63 \\
\hline
\end{tabular}

${ }^{a}$ The film thickness is in a range of 4-7 nm for all measurements

The FQR value is higher for MeOPI than $\mathrm{NO}_{2} \mathrm{PI}$ during the initial $60 \mathrm{~s}$ exposure but is lower for MeOPI than $\mathrm{NO}_{2} \mathrm{PI}$ after $60 \mathrm{~s}$. This time-dependent $\mathrm{FQR}$ is likely due to the different permeability of these two isocyanates in the polymer film. Since the gas permeation involves adsorption of the permeating species onto the polymer, diffusion through the polymer and desorption of the permeating species from the polymer surface and removal, the permeability can be affected by solubility and diffusivity of isocyanate molecules in the polymer film. At the 
beginning, the FQR is mainly controlled by the diffusion rate, which is governed by the vapor pressure. Therefore, MeOPI with a higher vapor pressure $(0.107 \mathrm{mmHg})$ gives a higher FQR than $\mathrm{NO}_{2} \mathrm{PI}\left(6.84 \times 10^{-3} \mathrm{mmHg}\right)$. At a later stage, the adsorption and desorption of isocyanate molecules in polymer reach an equilibrium and the $\mathrm{FQR}$ should now be governed by the isocyanate solubility in polymer. Thus, $\mathrm{NO}_{2} \mathrm{PI}$ with a higher affinity gives a larger FQR than MeOPI.

In summary, a conjugated fluorescent polymer has been successfully synthesized and used for direct detection of various isocyanates in air. Polymer P1 is highly sensitive to aromatic and aliphatic isocyanates with a detection limit at the ppt level. The isocyanate sensing under ambient conditions is fast, typically within a period of 10-60 s. A highly sensitive optical sensor based on $\mathbf{P 1}$ or other similar conjugated polymers is feasible and applicable for direct monitoring the isocyanate chemicals at a workplace. 


\subsection{References}

1 J. H. Saunders and R. J. Slocombe, Chem. Rev., 1948, 43, 203.

2 R. G. Arnold, J. A. Nelson and J. J. Verbanc, J. Chem. Edu., 1957, 34, 158.

3 M. Sato, J. Am. Chem. Soc., 1960, 82, 3893.

4 M. Kaplan, J. Chem. Eng., 1961, 6, 272.

5 R. B. Seymour and G. B. Kauffman, J. Chem. Ed., 1992, 69, 909.

6 Flexible polyurethane foams. Technical bulletin 2008, Doc. No. AX-239.

7 R. P. Streicher, C.M. Reh, R. Key-Schwartz, P. C. Schlecht and M. E. Cassinelli, NOISH Manual of Analytical Methods, 2004, 116.

8 A Summary of Health Hazard Evaluations: Issues Related to Occupational Exposure to Isocyanates, 1989 to $2002,2004,116$.

9 L. H. Kormos, R. L. Sandridge and J Keller, Anal. Chem., 1981, 53, 1122.

10 L. T. Budnik, D. Nowak, R. Merget, C. Lemiere and X. Baur, J. Occup. Med. Toxicol., $2011,6,9$.

11 M. M. Methner, C. Achutan and A. Adebayo, Health Hazard Evaluation Report, 20040349-2970 and the references there in.

12 See ref. 2 in ESI.

13 (a) C. J. Purnell and R. F. Walker, Analyst, 1985, 110, 893; (b) R. J. Rando and Y. Y. Hammad, Am. Ind. Hyg. Assoc. J., 1985, 46, 206.

14 (a) K. Marcali, Anal. Chem., 1957, 29, 552; (b) K. E. Grim and A. L. Linch, Am. Ind. Hyg. Assoc. J., 1964, 25, 285.

15 J. Keller, K. L Dunlap and R. L. Sandridge, Anal. Chem., 1974, 46, 1845.

16 J. Keller and R. L. Sandridge, Anal. Chem., 1979, 51, 1868. 
17 R. F. Walker and M. A. Pinches, Analyst, 1979, 104, 928.

18 L. H. Kormos, R. L. Sandridge and J Keller, Anal. Chem., 1981, 53, 1122.

19 W. E. Rudzinski, L. Pin, R. Sutcliffe, A. Richardson and T. Thomas, Anal. Chem., 1994, 66, 1664.

20 (a) S. Gagné, J. Lesage, C. Ostiguy, Y. Cloutier and H. V. Tra, J. Environ. Monit., 2005, 7, 145. (b) D. Karlsson, M. Spanne, M. Dalene and G. Skarping, Analyst, 1998, 123, 117.

21 B. Agarwal, S. Jürschik, P. Sulzer, F. Petersson, S. Jaksch, A. Jordan and T. D. Märk, Rapid Commun., Mass Spectrom. 2012, 26, 983.

22 S.W. Thomas, G. D. Joly and T. M. Swager, Chem. Rev., 2007, 107, 1339.

23 X. Duan, L. Liu, F. Feng and S. Wang, Acc. Chem. Res., 2009, 43, 260.

24 E. Clar, Chem. Ber., 1931, 64, 1676.

25 W. Theilacker, U. Berger-Brose and K.-H. Beyer, Chem. Ber., 1960, 93, 1658.

26 J.-S. Yang and T. M. Swager, J. Am. Chem. Soc., 1998, 120, 11864.

27 J.-S. Yang and T. M. Swager, J. Am. Chem. Soc., 1998, 120, 5321.

28 (a) J. Luo, Z. Xie, J. W. Y. Lam, L. Cheng, H. Chen, C. Qiu, H. S. Kwok, X. Zhan, Y. Liu, D. Zhu and B. Z. Tang, Chem. Commun., 2001, 1740; (b) B. Z. Tang, X. Zhan, G. Yu, P. P. S. Lee, Y. Liu and D. Zhu, J. Mater. Chem., 2001, 11, 2974.

29 B. Xu, Z. Chi, H. Li, X. Zhang, X. Li, S. Liu, Y. Zhang and J. Xu, J. Phys. Chem. C, $2011, \mathbf{1 1 5}, 17574$.

30 X. Fan, J. Sun, F. Wang, Z. Chu, P. Wang, Y. Dong, R. Hu, B. Z. Tang and D. Zou, Chem. Commun., 2008, 2989.

31 W.-E. Lee, C.-L. Lee, T. Sakaguchi, M. Fujikid and G. Kwak, Chem. Commun., 2011, 47, 3526. 
32 X. Zhou, H.-Y. Li, Z.-G. Chi, B.-J. Xu, X.-Q. Zhang, Y. Zhang, S.-W. Liu and J.-R. Xu, J. Fluoresc., 2012, 2, 565.

33 J. Chen, C. C. W. Law, J. W. Y. Lam, Y. Dong, S. M. F. Lo, I. D. Williams, D. Zhu and B. Z. Tang, Chem. Mater., 2003, 15, 1535.

34 H. Li, X. Zhang, Z. Chi, B. Xu, W. Zhou, S. Liu, Y. Zhang and J. Xu, Org. Lett., 2011, 13,556 .

35 W. Wu, S. Ye, L. Huang, L. Xiao, Y. Fu, Q. Qi Huang, G. Yu, Y. Liu, J. Qin, Q. Lia and Z. Li, J. Mater. Chem., 2012, $22,6374$.

36 R. Hu, J. L. Maldonado, M. Rodriguez, C. Deng, C. K. W. Jim, J. W. Y. Lam, M. M. F. Yuen, G. Ramos-Ortiz and B. Z. Tang, J. Mater. Chem., 2012, 22, 232.

37 Y. Liu, C. Deng, L. Tang, A. Qin, R. Hu, J. Z. Sun and B. Z. Tang, J. Am. Chem. Soc., $2011, \mathbf{1 3 3}, 660$.

38 T. Yamamoto and K. Takimiya, J. Am. Chem. Soc., 2007, 129, 2224.

39 X.-Z. Zhu and C.-F. Chen, J. Org. Chem., 2005, 70, 917.

40 Z. Zhu and T. M. Swager, Org. Lett., 2001, 3, 3471. 


\section{Chapter 5 Detection of Nitroaromatic Compounds by Fluorescent Conjugated Polymer}

\subsection{Introduction}

\subsubsection{Overview of nitroaromatic compounds}

Nitroaromatic compounds are generally less stable under certain conditions (temperature and pressure) and considered as explosives. An explosive compound is defined as an unstable material (chemical or nuclear) that can be initiated to undergo a very rapid, and self-propagating decomposition resulting in the formation of more stable material, the liberation of heat or the development of sudden pressure effect. Basically, explosives are classified as low and high; these two categories and both types are further classified into different sub-category as shown in Figure 5.1. ${ }^{1,2}$

Secondary explosives which include nitroaromatics and nitramines are extensively used in military sites. They are often used as main charge or bolstering explosives because they are formulated to detonate only under specific circumstances. ${ }^{1,2}$

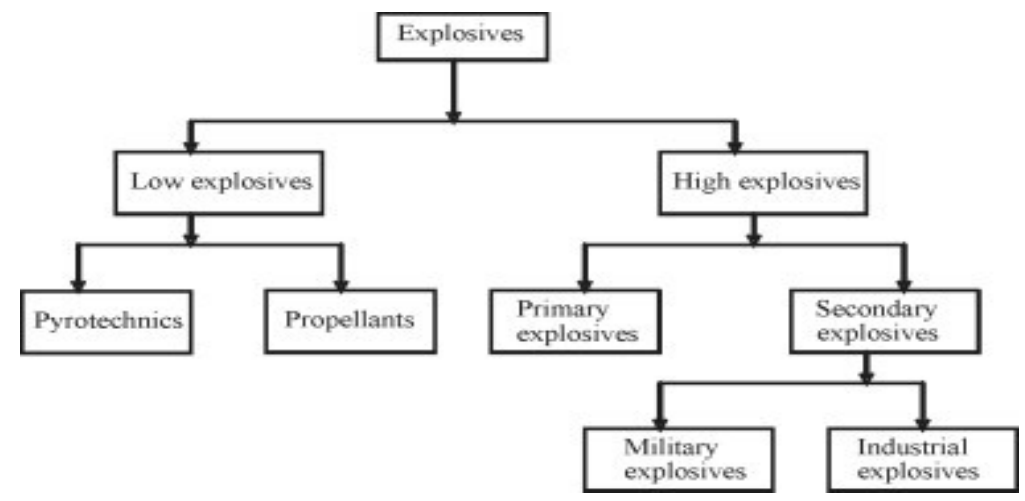

Figure 5.1 Classification of Explosives based on structure, performance, and sensitivity ${ }^{1}$

Nitroaromatic explosives such as trinitrotoluene (TNT) and picric acid (PA) are composed of a benzene ring-functionalized with several nitro groups. The detection of this class 
of explosives is not easy because they have moderate vapor pressures and their reactivity is quite limited. ${ }^{3}$ Most of the explosive compounds contain the nitro groups in the structures and are considered to be electron deficient. The electron-withdrawing group in an aromatic ring lowers the energy level of the empty $\pi^{*}$ orbital, thus making them a good electron acceptor. Therefore, the reduction potentials become less negative (more favorable) with increasing the nitro substituents. The reduction potential of TNT is $-0.7 \mathrm{~V}$ versus the normal hydrogen electrode (NHE), which is less negative than that of either DNT (-0.9 V versus NHE) or nitrobenzene ($1.15 \mathrm{~V}$ versus NHE). ${ }^{4,5}$ Most of the fluorescent conjugated polymers are highly electron rich which have a very high HOMO level, as a result they have been applied to detect the nitroaromatic explosives in the vapor phase. ${ }^{6}$ Therefore, a nonbonding electrostatic interaction between the electron deficient explosive and electron rich polymer is achieved which causes the fluorescence quenching. The fluorescence quenching mechanism can also be achieved through the Lewis acid-base (donor-acceptor or charge transfer) mechanism. Electron deficient nitro explosives act as electron acceptors for photo-excited electrons of the fluorescent polymers. These electron-deficient molecules are able to form $\pi$-stacking complexes with electron-rich fluorophores, and this particular property has been widely used for their detection with chromofluorogenic probes. ${ }^{7,8}$

In this part of the thesis, a few properties of the analyte were considered for detection: 1) electron deficiency 2) volatility 3) diffusion rate. The moderate vapor pressures of nitroaromatics make a perfect experimental analyte for detection in air using electron-rich conjugated polymers. However, these explosives in water (solution) are challenging for detection for fluorescence method using fluorescent conjugated polymers. Herein we chose picric acid (PA) and 2,4- 
dinitrotoluene (DNT) as representatives of nitroaromatic explosives for fluorescence detection in aqueous solution and in air, respectively.

\subsubsection{Contribution}

The following is a full version of the article "Ultra-Sensitive Detection of Explosive in Solution and Film as well as the Development of Thicker Film Effectiveness by Tetraphenylethene Moiety in AIE Active Fluorescent Conjugated Polymer" published on June 11, 2014 in Polymer Chemistry and reprinted with permission from The Royal Society of Chemistry Copyright Clarence Centre Appendix C). This includes detailed supporting information with optimization data and tables as well as additional unpublished data, provided therein. The synthesis of the tetraphenylethylene (TPE) monomer and polymerization were carried out by another student, Sukanta Kumar Saha. Manuscript writing, synthesis of monomer pentiptycene and rest of the experimental work including film preparation and sensitivity of the polymer towards PA and DNT as well as the related calculations were carried out by me.The link to the article: $\underline{\text { http://pubs.rsc.org/en/content/articlelanding/2014/py/c4py00673a\#!divAbstract }}$

\subsubsection{Abstract}

A fluorescence sensory polymer consisting of pentiptycene and tetraphenylethylene (TPE) units linked by acetylene was synthesized for direct detection of explosives in solution and air. At the same time, the effectiveness of thicker film in the presence of TPE moiety for the detection of explosive was investigated. Fluorescence turn-off detection of PA was carried out in $\mathrm{H}_{2} \mathrm{O} /$ THF mixture $\left(\mathrm{H}_{2} \mathrm{O} /\right.$ THF) containing $90 \%$ water, where the TPE units of the polymer are in a highly emissive aggregation state. Polymer P1 exhibits a fluorescence quenching response 
(FQR) of $24 \%$ for $4.36 \times 10^{-8} \mathrm{M}$ of PA and $98 \%$ for $4.7 \times 10^{-6} \mathrm{M}$ of PA. For a thin film (4 nm) of P1 after exposure to DNT, its fluorescence intensity dropped $48 \%$ after $10 \mathrm{~s}$ and continued to drop $97 \%$ after $300 \mathrm{~s}$. In comparison, the fluorescence intensity decreased $29 \%$ (10 s) and $72 \%$ (300 s) for a thicker film $(75 \mathrm{~nm})$. These results indicate that $\mathbf{P 1}$ is a promising material for direct detection of nitroaromatic explosives in air and in solution at a ppb level. The TPE moiety in the polymer improves the sensitivity and effectiveness of thick polymer films for sensing. 


\subsubsection{References}

1 Singh, S. J. Hazard. Mater. 2007, 144, 15.

2 Hoor, M. J. T. J. Chem. Ed. Chem. Wisc. Edu. 2003, 80, 1397.

3 Oxley, J. C.; Smith, J. L.; Shinde, K.; Moran, J. Propellants, Explosives, Pyrotechnics, 2005, 30, 127.

$4 \quad$ Yang, J.-S.; Swager, T. M. J. Am. Chem. Soc. 1998, 120, 11864.

5 Sohn, H.; Calhoun, R. M.; Sailor, M. J.; Trogler, W. C. Angew. Chem. Int. Ed. 2001, 40, 2104

6 McQuade, D. T.; Pullen, A. E.; Swager, T. M. Chem. Rev. 2000, 100, 2537.

7 Salinas, Y.; Martınez-Manez, R.; Marcos, M. D.; Sancenon, F.; Costero, A. M.; Parra, M.; Gil, S. Chem. Soc. Rev. 2012, 41, 1261.

8 He, C.; Zhu, D.; He, Q.; Shi, L.; Fu, Y.; Wen, D.; Cao, H.; Chen, J. Chem. Commun. 2012, $48,5739$. 


\subsection{Article}

Nitroaromatic compounds are commonly used as explosives among them 2,4,6trinitrotoluene (TNT) is a main component in more than 10 types of bombs, especially those used in landmines. ${ }^{1}$ The recent global terrorism has made an attention to trace detection of explosives. $^{2}$ Low-level exposure to PA, DNT and TNT can cause eye injury, red skin, liver damage and aplastic anemia. ${ }^{3}$ The allowable contaminate level of PA is not estimated; that of DNT is $0.2 \mu \mathrm{g} \mathrm{L} \mathrm{L}^{-1}$ and TNT in water is $2 \mathrm{ppb}^{4}$ This implies that the detection of explosive materials is greatly required for stopping unexpected terrorist activities and to ensure safe living. For the detection of explosives, there are many different techniques are available like gas chromatography coupled with mass spectrometry, ${ }^{5,6}$ surface-enhanced Raman spectroscopy, ${ }^{7}$ ion mobility spectrometry, ${ }^{8}$ energy dispersive X-ray diffraction, ${ }^{9}$ metal detectors. ${ }^{7}$ Though among the above-mentioned methods some are highly sensitive to the explosives, they are expensive, need to be calibrated frequently, require time-consuming procedures, ${ }^{10}$ or too sophisticated to handle that are not easily applied to the on-field testing. ${ }^{11}$ Besides these methods for the onsite explosive detection, the use of dogs is one of the most common methods, but is totally dependent on the behavior of the dogs, and also the training and long-term care of dogs are expensive. ${ }^{12}$ So, the development of efficient and cost-effective detection devices has become an urgent worldwide necessity.

Over the last two decades, considerable research efforts have been devoted to fluorescence sensor polymers for accurate and rapid detection of nitro explosives by Swager and many other research groups, using pentiptycene, modified pentiptycene and tetraphenylethylene (TPE) moieties as the key components in conjugated polymers. ${ }^{2,13,14}$ Among them, several pentiptycene based conjugated polymers are found to be highly sensitive towards nitroaromatic explosives. ${ }^{2,13}$ We previously reported a series of highly sensitive sensory polymers containing 
pendent cholesteryl ester groups followed by fluorene and pentiptycene for detection of nitroaromatics. ${ }^{15}$

TPE-based polymers are Aggregation-Induced Emission (AIE) active, AIE being a phenomenon where a non-emissive material in solution becomes highly luminescent upon aggregation. ${ }^{16}$ Recently, TPE-based conjugated polymers for sensing of nitro explosives have become promising materials. Most of them are demonstrated in solution with a rare example in the film.

Pentiptycene has a three-dimensional rigid and bulky structure, which prevents $\pi$ stacking or excimer formation in the excited state in the solid film; as a result, a gaseous analyte can easily interact with the surface and diffuse through the film causing fluorescence quenching. ${ }^{13 \mathrm{a}, \mathrm{d}}$ TPE polymer in solution forms highly emissive nanoaggregates; an analyte can enter the nanoaggregates and be captured, at the same time associating with the polymer by nonbonding electrostatic interactions or potential involvement of Lewis acid-base interactions, causing fluorescence quenching. ${ }^{14 a, b}$

To detect an explosive in air with rapid response and a high sensitivity, a fluorescence polymer film that features a high degree of conjugation, large free volume, and high adsorption ability of gaseous analyte on the surface, with a fast diffusion rate and high solubility through the film would be an ideal system. Moreover, to detect an explosive in solution with rapid response and a high sensitivity, a fluorescence polymer that features strong luminescent amplification characteristics, e.g., AIE enhancement with high degree of conjugation, would be an ideal system.

Since nitroaromatic compounds are electron deficient in nature, with highly conjugated electron-rich polymers are able to make $\pi$-stacking complexes, and by the photoinduced electron 
transfer process, the fluorescence intensity of polymer sensors can be quenched. ${ }^{17,18}$ Highly fluorescent AIE active polymers are also able to be quenched by nitroaromatics through energy transfer. With these basic requirements in mind, we have designed a polymer (P1) which consists of pentiptycene diacetylene (1) and 1,2-bis(4-bromophenyl)-1,2-diphenylene (2) as monomers making the polymer highly conjugated and electron rich. This polymer showed high sensitivity towards isocyanates and can detect them at ppt levels. ${ }^{19}$ Though isocyanates are less electron deficient than the explosive compounds, we expect polymer P1 to have very high sensitivity towards nitroaromatic explosives. In this paper, we report the detection of nitroaromatic compounds in air and solution as well as the effectiveness of a thicker polymer film. We also hypothesized that TPE moiety would increase the cavity of the film as well as conjugation, thus enhancing the performance of the thicker film.

\section{Measurements}

${ }^{1} \mathrm{H}$ NMR and ${ }^{13} \mathrm{C}$ NMR spectra were recorded on a Bruker Avance Digital 300 and 400 $\mathrm{MHz}\left(300,400\right.$ and 75, $100 \mathrm{MHz}$ for ${ }^{1} \mathrm{H}$ NMR and ${ }^{13} \mathrm{C}$ NMR, respectively). Resonances were quoted on the $\delta$ scale relative to tetramethylsilane (TMS, $\delta=0$ ) as an internal standard. For ${ }^{1} \mathrm{H}-$ NMR spectra, the following abbreviations have been used: $\mathrm{s}=$ singlet, $\mathrm{d}=$ doublet, $\mathrm{t}=$ triplet, $\mathrm{q}=$ quartet, $\mathrm{m}=$ multiplet. Infrared measurements were performed on a Varian 1000 FT-IR Scirinitar series spectrophotometer. The following abbreviations have been used in IR spectra: $\mathrm{s}=$ strong, $\mathrm{m}=$ medium, $\mathrm{w}=$ weak, $\mathrm{br} .=$ broad, $\mathrm{sh}=$ sharp. The measuring mode was $\% \mathrm{~T}$ (percentage transmittance). The band positions were expressed in per centimeter $\left(\mathrm{cm}^{-1}\right)$. Mass spectra were measured with a Micromass Quattro LC ESI (EI). The UV-Vis spectra were recorded with a Perkin-Elmer Lambda 900 UV-Vis-NIR spectrometer at room temperature. The fluorescence 
spectra of all samples were measured in a quartz cuvette with a path length $1 \mathrm{~cm}$ in Shimadzu RF-1501 spectrofluorometer.

\section{Preparation of fluorometric titration solutions}

Stock THF solutions of polymer P1 with a concentration of $1.17 \times 10^{-3} \mathrm{M}$ in $10 \mathrm{~mL}$ was prepared and then $1 \mathrm{~mL}$ of the solution was transferred into a $20 \mathrm{~mL}$ vial to further dilution by 9 $\mathrm{mL}$ of THF as experiment solution. After adding an appropriate amount of the experiment solution into a $20 \mathrm{~mL}$ vial, water was added under vigorous stirring to prepare $\mathrm{H}_{2} \mathrm{O}$-THF mixture of different fractions $\left(f_{w}=0-95\right)$ by volume. A stock PA solution was prepared at a concentration of $4.36 \times 10^{-3} \mathrm{M}$ in $10 \mathrm{~mL}$ THF. The fluorescence spectroscopic titration was carried out by sequentially adding $0.1,0.2,0.3$ and up to $10.8 \mu \mathrm{L}$ of PA solution to $10 \mathrm{~mL}$ of $\mathbf{P 1}$ in $\mathrm{H}_{2} \mathrm{O}-\mathrm{THF}$ mixture containing $90 \%$ of water. The solution of P1 with PA was stirred for 1 minute and then placed in a quartz cuvette. The fluorescence spectra of the resultant mixtures were then recorded immediately at room temperature with excitation wavelength of $365 \mathrm{~nm}$. During the titrations the instrument sensitivity was kept at low, otherwise, it went beyond the range.

\section{Film formation}

Films coated on glass substrates were prepared by the spin-coating process. Polymer films were spin-coated from 1,1,2,2 tetrachloroethane solutions onto a glass plate (microscope slide, $20 \times 20 \times 1 \mathrm{~mm}$ ) and spun at $1000 \mathrm{rpm}$ by using a Chemat KW-4B spin-Coater. Then the films were dried for overnight at $80{ }^{\circ} \mathrm{C}$ under argon atmosphere. To prepare $4-80 \mathrm{~nm}$ thick films, 5-10 mg of polymer in $1.5 \mathrm{~mL}$ of solvent were used. The thickness of the films ranged from 4-80 
nm, as measured using a Tencor Instrument Alpha-Step 200 surface profiler (uncertainty \pm 0.01 $\AA$ ) and by Atomic Force Microscopy (AFM) (uncertainty $\pm 0.2 \mathrm{~nm}$ ).

The fluorescence spectra were recorded with a fluorimeter immediately after exposing the polymer film to the analyte, 2,4-DNT for a specific period of time. Fluorescence Quenching Response (FQR) is defined as $\mathrm{I}_{0}-\mathrm{I} / \mathrm{I}_{0} \times 100$, where $\mathrm{I}_{0}$ and $\mathrm{I}$ are the fluorescence intensity prior to and after exposure to analyte (DNT) vapor, respectively.

\section{Results and discussion}

The synthetic route to the target sensory polymer P1 is shown in Scheme 1. The Sonogashira cross-coupling reaction of compound $\mathbf{1}$ with compound $\mathbf{2}$ affords polymer $\mathbf{P} \mathbf{1}$ in $71 \%$ yield which we have recently published. Its characterization by spectroscopic method has been mentioned in that literature. ${ }^{19}$
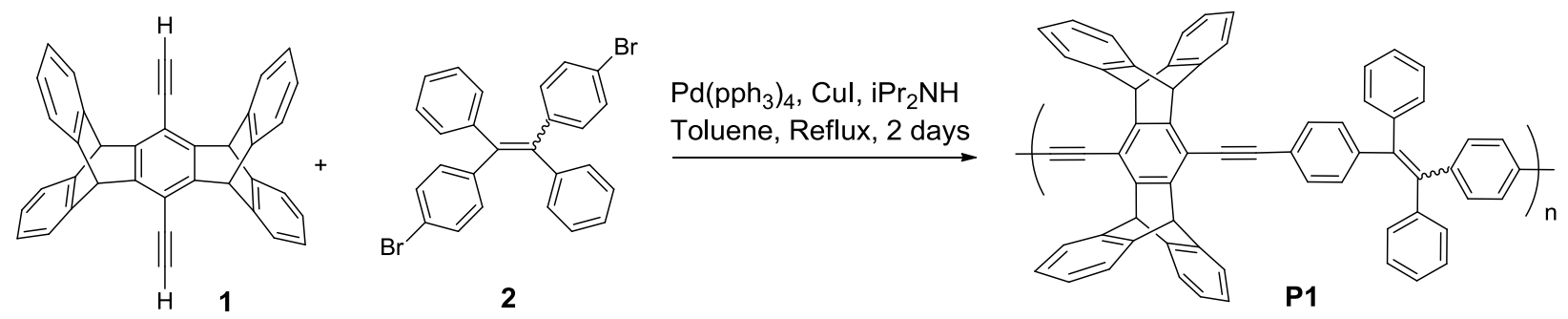

Scheme 1. Synthesis of polymer $\mathbf{P 1}$ by Sonogashira cross-coupling reaction

The backbone of polymer P1 contains the TPE unit, which is AIE active. The emission property of polymer and its AIE behavior were investigated in THF and in $\mathrm{H}_{2} \mathrm{O}$-THF mixture solutions (Fig. 1). Tang has revealed that TPE-containing polyenes are non-emissive in solution but become luminescent afterwards due to AIE with the addition of poor solvent. This emission gradually increased with the formation of aggregates and reached its maximum in $90 \%$ aqueous solution. ${ }^{14 a, b, 16}$ A similar phenomenon was observed for polymer $\mathbf{P 1}$ in THF and in $\mathrm{H}_{2} \mathrm{O}-\mathrm{THF}$ 
solutions. Polymer P1 is non-emissive or very weakly emissive in THF or in $\mathrm{H}_{2} \mathrm{O}-\mathrm{THF}$ mixture when the water content is below $30 \%$, but upon further addition of water the photoluminescence (PL) intensity gradually increases at around $490 \mathrm{~nm}$.

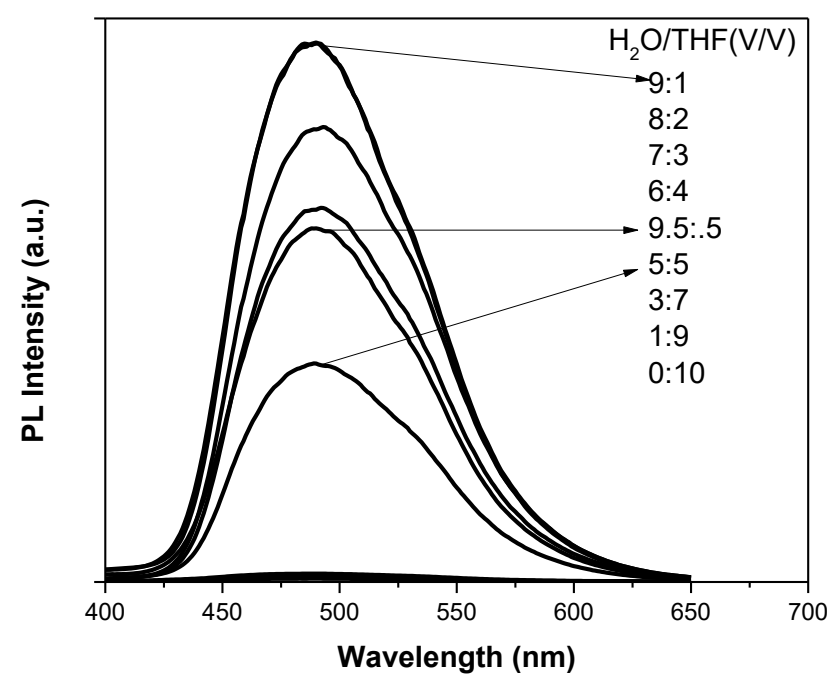

Fig. 1 Photoluminescence spectra of polymer $\mathbf{P 1}$ in $\mathrm{H}_{2} \mathrm{O}-\mathrm{THF}$ mixtures with different water contents. Excitation wavelength: $365 \mathrm{~nm}$.

The photograph in Fig. S1 clearly indicates that in $\mathrm{H}_{2} \mathrm{O}-\mathrm{THF}$ solution with lower water fraction, polymer P1 emits no light; the emission becomes stronger afterwards due to the aggregation.

\section{Explosive detection by polymer nano-aggregates}

We used picric acid (PA) as a model explosive of TNT in this work. The sensitivity of polymer P1 towards PA was determined by fluorescence spectroscopic titration experiment. Fig. 2 displays the fluorescence turn-off detection of different concentrations of PA using P1 (1.17x $10^{-5} \mathrm{M}$ ) in $\mathrm{H}_{2} \mathrm{O}-\mathrm{THF}$ mixture containing $90 \%$ of water. Upon the addition of PA into P1 
aggregates solution, the emission intensity decreased quickly and FQR reached over $98 \%$ with the PA concentration at $4.71 \times 10^{-6} \mathrm{M}$. The inset photograph (bottom) shows that polymer $\mathbf{P 1}$ is highly emissive but after addition of PA emission decreases, which is also detectable by the naked eye (inset photograph (top)).

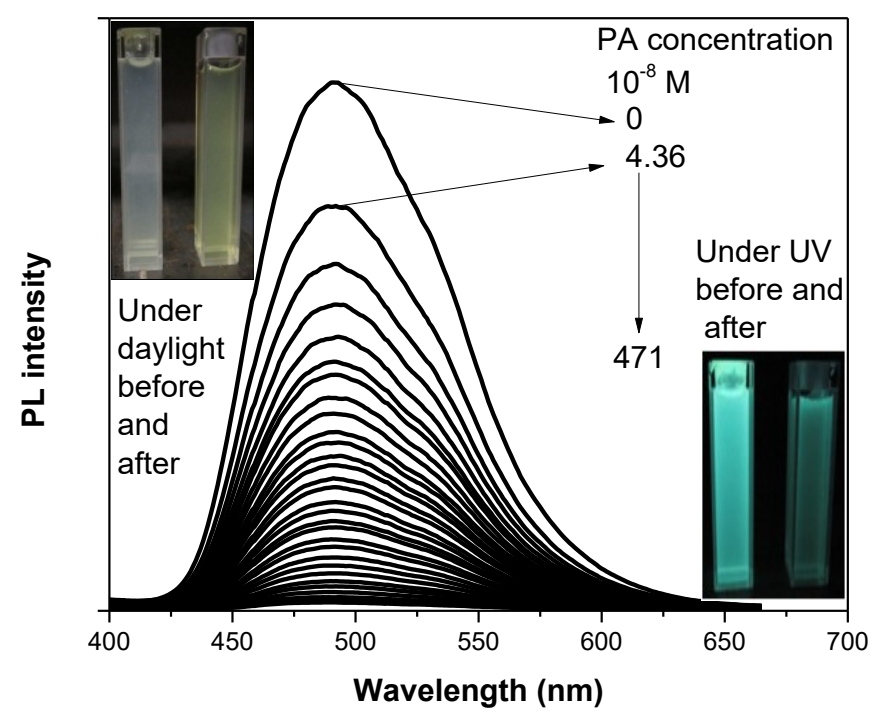

Fig. 2 Fluorescence response of $\mathbf{P 1}\left(1.17 \times 10^{-5} \mathrm{~mol} / \mathrm{L}\right)$ to the addition of different amounts of picric acid (PA) into the $\mathrm{H}_{2} \mathrm{O}-\mathrm{THF}$ mixture solution with a water fraction of $90 \%$. Excitation wavelength: $365 \mathrm{~nm}$.

The FQR was $24 \%$ with addition of $4.36 \times 10^{-8} \mathrm{M}$ PA to the nano-aggregates of $\mathbf{P 1}$, which confirmed the nanoaggregates of $\mathbf{P} \mathbf{1}$ could be a very sensitive sensor for the detection of nitroaromatic explosives at the ppb level in solution. Fig. S3 reveals that polymer P1 exhibits high sensitivity towards PA with the association constant between the host and guest being $37 \times$ $10^{-8} \mathrm{M}^{-1}$, deduced by the Stern-Volmer equation, which is lower than that reported for TPE-based fluorescence sensor polymers. ${ }^{14 a, b}$ This can be explained by the fact that in the turn-off detection 
system where water content is high, the AIE active TPE units are in aggregated state, which gives intense fluorescence. In addition, pentiptycene is a large compound and maintains a large free volume inside the polymer allowing the analyte to enter into the polymer. This large cavity in the nano-aggregated state helps to bind with more PA thus making the quenching a highly efficient process for polymer $\mathbf{P 1}{ }^{17,} 20$ This can also be explained by the Lewis acid-base interaction between the electron-rich polymer P1 and the electron-deficient PA molecules as well as the photoinduced electron transfer from the higher lowest unoccupied molecular orbital (LUMO) energy level of P1 nanoaggregates to PA which plays a key role in the quenching process. Cyclic Voltammetry (CV) was carried out for this polymer to determine the highest occupied molecular orbital (HOMO) and LUMO energy levels (Fig. S4 in SI). From CV we only got the noticeable oxidation process $\left(0.87 \mathrm{~V}\right.$, onset ${ }^{1}$ since our synthesized polymer is electron rich. This oxidation potential value provides the HOMO energy level of $-5.25 \mathrm{eV} .^{21}$ Calculation of LUMO from optical energy band gap tends to give a large error; therefore, the calculated LUMO would not be accurate. Addition of trace analytes to the polymer aggregates leads to quenching, indicating that surely the LUMO of the polymer is much higher than the LUMO of analytes (PA and DNT) which facilitates electron transfer from the excited polymer to analytes. Fig. S5 in the SI illustrates that there is an overlap between the absorption spectrum of PA and the emission spectrum of the polymer aggregates, but this was not possible for DNT. Therefore, in solution the quenching mechanism can be dominated by charge or energy transfer or both, depending on the overlap of the absorption of nitroaromatics and emission of polymer at the highest aggregation state. ${ }^{14 \mathrm{c}, 14 \mathrm{e}, 22}$

\footnotetext{
${ }^{1}$ There was an error in the article, the onset oxidation would be $0.87 \mathrm{~V}$ instead of $2.02 \mathrm{~V}$ and the calculated HOMO would be $-5.25 \mathrm{eV}$ instead of $6.81 \mathrm{eV}$
} 


\section{Study of fluorescence quenching of polymer P1 film by DNT}

DNT was used as an analyte in our study, because of its higher equilibrium vapor pressure $\left(1.47 \times 10^{-4} \mathrm{mmHg}\right.$ at $\left.22{ }^{0} \mathrm{C}\right)$ than TNT $\left(8.06 \times 10^{-6} \mathrm{mmHg} \text { at } 25{ }^{0} \mathrm{C}\right)^{7}$ as well as its availability. Fig. 3 shows the changes in fluorescence intensity of the P1 film (16 nm thick) upon exposure to DNT vapor over several different time periods. With a $10 \mathrm{~s}$ exposure, fluorescence intensity dropped $43 \%$ and after $360 \mathrm{~s}$ intensity dropped $92.0 \%$.

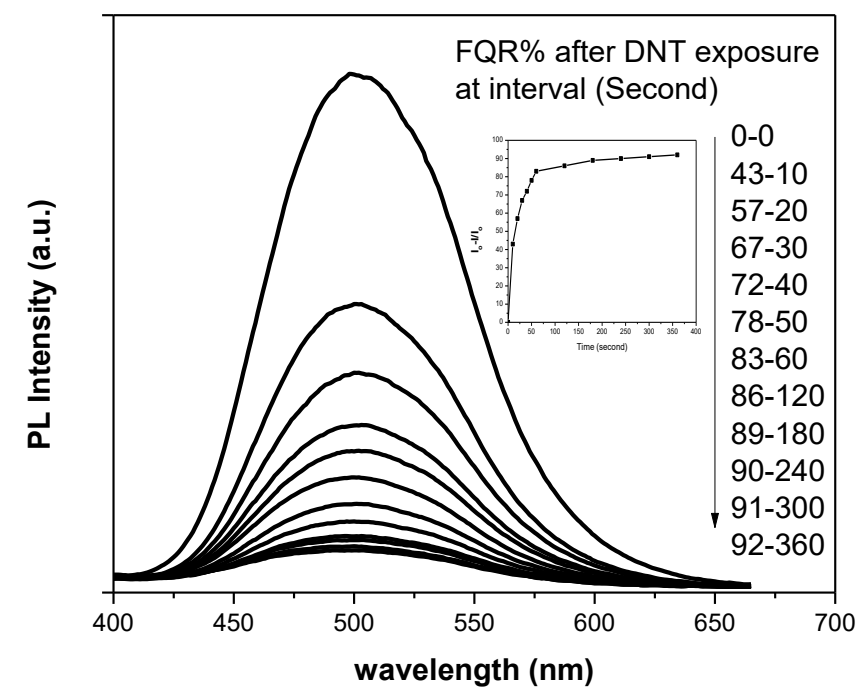

Fig. 3 Time-dependent PL spectra of a spin-coating film of $\mathbf{P 1}$ upon exposure to DNT vapor at room temperature for 0-360 s. Film thickness: $16 \mathrm{~nm}$; excitation wavelength: $375 \mathrm{~nm}$. 


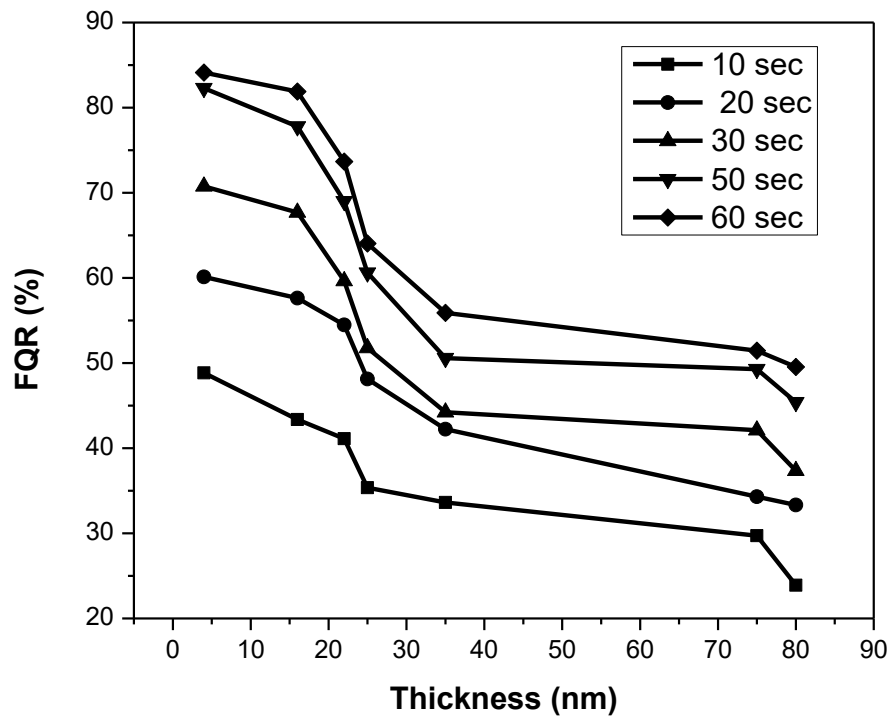

Fig. 4 Fluorescence quenching response of films of $\mathbf{P 1}$ of different thickness after exposure to DNT vapour for 10-60 s.

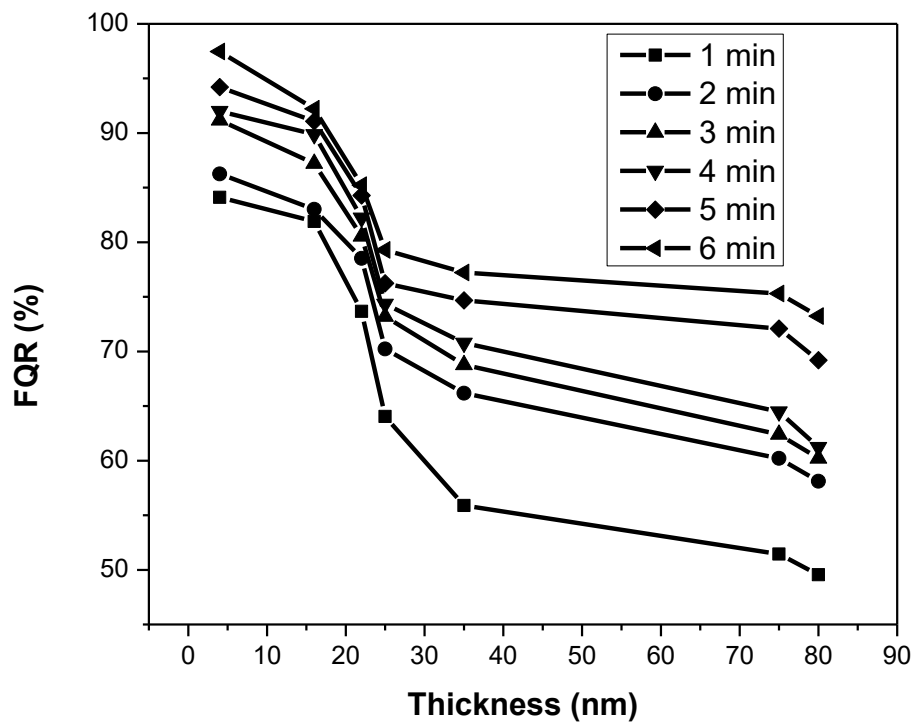

Fig. 5 Fluorescence quenching response of films of $\mathbf{P} \mathbf{1}$ of different thicknesses after exposure to DNT vapor for 1-6 min. 
Fig. 4 and 5 display the FQR for different time intervals upon exposure of DNT analyte to different thicknesses of the polymer film. For a $4 \mathrm{~nm}$ film, the FQR after exposures for $30 \mathrm{~s}$ is $70 \%$; however, this reduces to $37 \%$ for a film of $80 \mathrm{~nm}$ in thickness. This result is consistent with the time intervals within $60 \mathrm{~s}$ exposure to DNT vapor: our experimental work shows that thinner films give a higher response than thicker films, especially in the first $30 \mathrm{~s}$. A similar phenomenon was observed for 1-5 min of DNT exposure (Fig. 5). However, after 5 min exposure to DNT, films around $35-75 \mathrm{~nm}$ in thickness gave almost the same FQR (77-73\%). This rapid response to the analyte indicates that the DNT molecules have better nonbonding electrostatic interactions with the electron-rich polymer. ${ }^{9}$ Thinner films $4-30 \mathrm{~nm}$, show a better FQR (85-64\%) within $60 \mathrm{~s}$ for our synthesized polymer P1. These results can be explained by the cavity of the polymer backbone and diffusion rate of the analyte through the polymer in the solid state. The pentiptycene and TPE moieties make the polymer more electron rich, thus leading to a high FQR within a short period of exposure. These two moieties also create a large number of cavities or free volume in the polymer film which permits a high diffusion rate for explosive molecules to enter and solubilize in the polymer film, thus gives a window for higher FQR in a longer period of exposure. Moreover, the rigid pentiptycene moiety in the polymer prevents significant $\pi$ stacking interactions between polymer backbones, thereby weakening the interpolymer interactions. As a result, the polymer will provide a very quick response regardless of thickness and, due to the morphological change in the polymer film; it will provide a better response with time for thicker films. Experimentally, the fluorescence signals decrease noticeably within only $10 \mathrm{~s}$ of DNT exposure regardless of the thickness of the film and reach equilibrium after $5 \mathrm{~min}$. The FQR value is always higher for thinner films and also it improves for thicker films with time, which indicates that the DNT molecules have strong interactions with film surface and can 
easily diffuse and solubilize through the bulk of the film. These results indicate that the TPE moiety enhances the formation of cavities along with the pentiptycene moiety in the solid state as well as increasing the conjugation, thus significantly improving the FQR for thicker films, though the thinner films have higher FQR. Our experimental result was relevant to our hypothesis and gave a better response than in our previous work. ${ }^{16}$ Therefore; our polymer is more suitable for the detection of nitroaromatic vapor.

\section{DNT detection study by using APTES under-coated and top-coated films of P1}

Fig. 6 displays the FQR for different time intervals upon exposure of DNT analyte to different thicknesses of polymer film under-coated by (3-amino propyl)triethoxysilane (APTES). APTES is a surface promoter for a variety of substances such as glass, silicon and plastics, and is able to form an ultrathin layer containing the free amino groups on the surface. The polar electron-donating group $\left(-\mathrm{NH}_{2}\right)$ in APTES has an ability to bind electron-deficient nitroaromatic compounds through the electron donor and acceptor interaction. The purpose of using APTES in our experiment was to get a quick response compared to the uncoated polymer film. However, Fig. 6 clearly shows that the polymer films with the APTES undercoating gave lower FQR than those without the undercoating for different time intervals. Thus, this indicates that the undercoating decreases the sensitivity of polymer P1. Fig. 7 displays the fluorescence turn-off detection of DNT using P1 top-coated with APTES. This shows lower FQR for different time intervals compared to the uncoated film, indicating that top coating also decreases the sensitivity of polymer P1.

Table 1 shows the level of fluorescence quenching after $10 \mathrm{~s}, 30 \mathrm{~s}$ and $60 \mathrm{~s}$ for a $4 \mathrm{~nm} \mathrm{P1}$ film with under-coated and top-coated surfaces with respect to that without APTES. There is a 
noticeable difference among them; initially, the under-coated film shows better response upon exposure than the top-coated one. After $60 \mathrm{~s}$ exposure of DNT vapor the top-coated film, it shows higher FQR compare to the undercoated film. The polymer film without APTES gave higher FQR response for time intervals than that with APTES coating. This indicates that the polymer itself has enough cavities to allow sufficient diffusion of DNT vapor through the film. Moreover, with the presence of APTES, the interaction with the analyte is more hindered, which leads to a slight decrease of FQR.

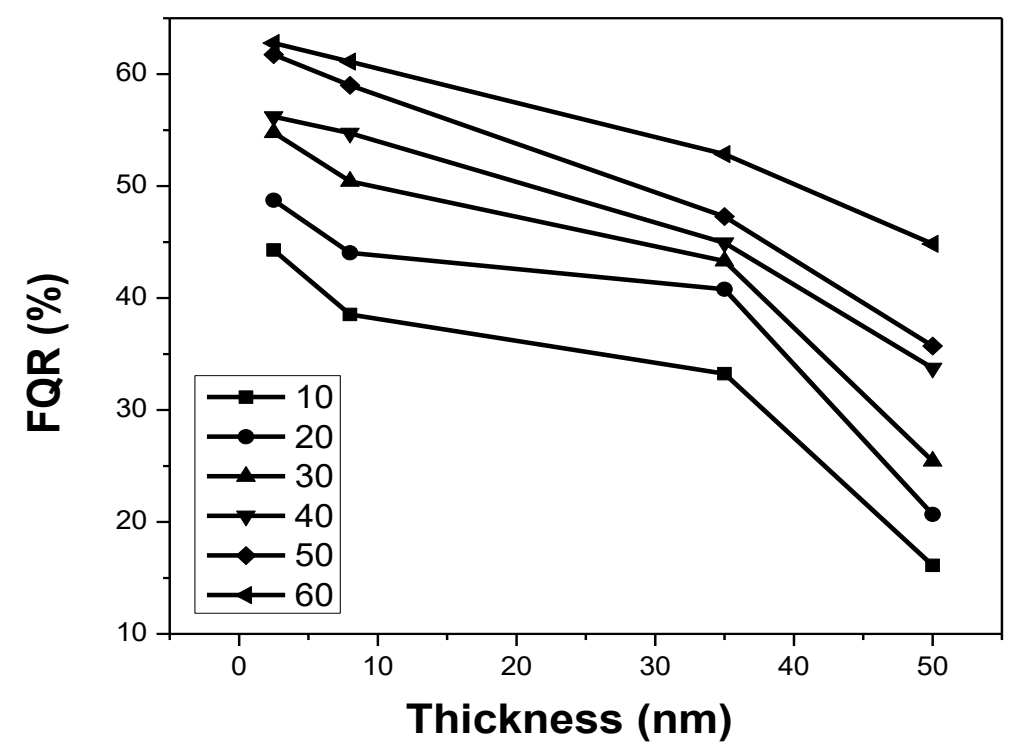

Fig. 6 Fluorescence quenching response of films of $\mathbf{P 1}$ of different thickness undercoated with APTES after exposure to DNT vapor for 10-60 s. 


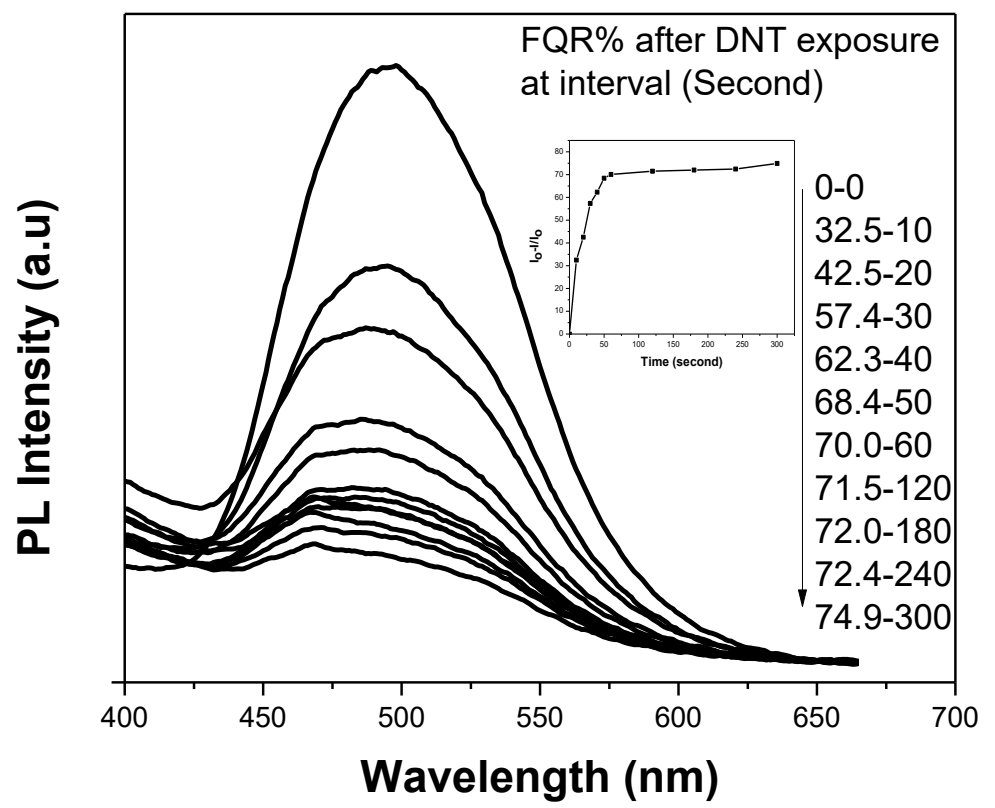

Fig. 7 Time-dependent PL spectra of the spin-coated film of P1 with APTES top-coating upon exposure to DNT vapor at room temperature for 0-300 s. Film thickness: $4 \mathrm{~nm}$; excitation wavelength: $375 \mathrm{~nm}$.

Table 1 Comparison of the fluorescence quenching response (FQR) of P1 films (4 nm) on glass plates with APTES under-coating and top-coating

\begin{tabular}{|c|c|c|c|}
\hline Time (s) & 10 & 30 & 60 \\
\hline (a) With under-coated film & 10.31 & 20.33 & 23.75 \\
\hline (b) With top-coated film & 16.38 & 13.41 & 14.79 \\
\hline
\end{tabular}

(a) Change in FQR $=\mathrm{F} 1-\mathrm{F} 2$, where $\mathrm{F} 1$ is the FQR without undercoating and F2 is the FQR with APTES undercoating

(b) Change in FQR $=F 1-F 2$, where $F 1$ is the FQR without top-coating and F2 is the FQR with APTES top-coating

\section{Conclusion}

In this work, a conjugated fluorescent polymer (P1) consisting of pentiptycene and TPE moieties that are linked with acetylene was successfully used as an explosive chemosensor both 
in the aggregated and solid states. Polymer P1 showed high sensitivity towards nitroaromatic explosives. In the aggregated state the detection limit can reach the ppb level; while for a solid film with a thickness of $4 \mathrm{~nm}, 85 \% \mathrm{FQR}$ has been recorded within $60 \mathrm{~s}$. Moreover, in the presence of TPE moiety, the polymer itself has enough cavities that facilitate better FQR for thicker films as well. The presence of top-coating or under-coating did not improve the FQR response. The experimental data indicated that $\mathbf{P 1}$ self-supporting films can be used as a promising sensor material for the detection of nitroaromatic explosives without the need for any further film modifications. 


\subsection{References}

1 W.R. Davidson, W. R. Scott, R. Sleeman, and A. k. Akery, Proc. SPIE, 1994, 108, 2092.

2 Y. Salinas, R. Martinez-Manez, M. D. Marcos, F. Sancenon, A. M. Costero, M. Parra and S. Gil, Chem. Soc. Rev., 2012, 41, 1261-1296.

3 U.S. departments of health and human services, Public Health Service Agency for Toxic Substances and Disease Registry, September 1996.

4 United state environmental protection agency, EPA 505-F-10-010, February 2010. (b) United state environmental protection agency, technical fact sheet-dinitrotoluene, EPA 505-F-14-010, January 2014.

5 J. Yinon, Forensic and Environmental Detection of Explosives; John Wiley \& Sons: Chichester, England, 1999.

6 J. Yinon, and S. Zitrin, Modern Methods and Applications in Analysis of Explosives; John Wiley \& Sons: Chichester, England, 1993

7 J. M. Sylvia, J. A. Janni, J. D. Klein and K. M. Spencer, Anal. Chem., 2000, 72, 58345840.

8 K. Hakansson, R. V. Coorey, R. A. Zubarev, V. L. Talrose and P. Hakansson, J. Mass Spectrom., 2000, 35, 337-346.

9 P. Kolla, Angew. Chem. Int. Ed. Engl., 1997, 36, 800-811.

10 S. Singh, J. Hazard. Mater. 2007, 144, 15-28.

11 Y. Engel, R. Elnathan, A. Pevzner, G. Davidi, E. Flaxer and F. Patolsky, Angew. Chem. Int. Ed. Engl., 2010, 49, 6830-6835.

12 D. S. Moore, Rev. Sci. Instrum., 2004, 75, 2499-2512. 
13 (a) J.-S. Yang and T. M. Swager, J. Am. Chem. Soc., 1998, 120, 5321-5322; (b) C. J. Cumming, C. Aker, M. Fisher, M. Fox, M. J. la Grone, D. Reust, M. G. Rockley, T. M. Swager, E. Towers and V. Williams, IEEE Trans. Geosci. Rem. Sens., 2001, 39, 11191128; (c) S. J. Toal and W. C. Trogler, J. Mater. Chem., 2006, 16, 2871-2883. (d) J.-S. Yang and T. M. Swager, J. Am. Chem. Soc., 1998, 120, 11864-11873; (e) S. Yamaguchi and T. M. Swager, J. Am. Chem. Soc., 2001, 123, 12087-12088; (f) S. Zahn and T. M. Swager, Angew. Chem., Int. Ed., 2002, 41, 4226-4230; (g) J. P. Amara and T. M. Swager, Macromolecules, 2005, 38, 9091-9094; (h) D. Zhao and T. M. Swager, Macromolecules, 2005, 38, 9377-9384; (I) S. W. Thomas III, J. P. Amara, R. E. Bjork and T. M. Swager, Chem. Commun., 2005, 4572-4574; (j) S. Rochat and T. M. Swager, ACS Appl. Mater. Interfaces, 2013, 5, 4488-4502.

14 (a) W. Wu, S. Ye, L. Huang, L. Xiao, Y. Fu, Q. Huang, G. Yu, Y. Liu, J. Qin, Q. Li and Z. Li, J. Mater. Chem., 2012, 22, 6374-6382. (b) R. Hu, J. L. Maldonado, M. Rodriguez, C. Deng, C. K. W. Jim, J. W. Y. Lam, M. M. F. Yuen, G. Ramos-Ortiz and B. Z. Tang, J. Mater. Chem., 2012, 22, 232-240 (c) J. Wang, J. Mei, W. Yuan, P. Lu, A. Qin, J. Sun, Y. Ma and B. Z. Tang, J. Mater. Chem., 2011, 21, 4056-4059 (d) W. Wu, S. Ye, G. Yu, Y. Liu, J. Qin and Z. Li, Macromol. Rapid Commun., 2012, 33, 164-171 (e) B. Xu, X. Wu, H. Li, H. Tong and L. Wang, Macromolecules, 2011, 44, 5089-5092.

15 (a) H. H. Nguyen, X. Li, N. Wang, Z. Y. Wang, J. Ma, W. J. Bock, and D. Ma, Macromolecules, 2009, 42, 921-926. (b) Z. Wang, Z, Y. Wang, J. Ma, W. J. Bock and D. Ma, Polymer, 2010, 51, 842-847. 
16 (a) J. Luo, Z. Xie, J. W. Y. Lam, L. Cheng, H. Chen, C. Qiu, H. S. Kwok, X. Zhan, Y. Liu, D. Zhu and B. Z. Tang, Chem.Commun., 2001, 1740-1741; (b) B. Z. Tang, X. Zhan, G. Yu, P. P. S. Lee, Y. Liu and D. Zhu, J. Mater. Chem., 2001, 11, 2974-2978.

17 S. W. Thomas III, G. D. Joly and T. M. Swager, Chem. Rev., 2007, 107, 1339-1386.

18 Q. Zhou and T. M. Swager, J. Am. Chem. Soc., 1995, 117, 12593-12602.

19 K. R. Ghosh, S. K. Saha, J. P. Gao and Z. Y. Wang, Chem. Commun., 2014, 50, 716-718.

20 (a) U. H. F. Bunz, Chem. Rev., 2000, 100, 1605-1644; (b) J. Liu, Y. Zhong, P. Lu, Y. Hong, J. W. Y. Lam, M. Faisal, Y. Yu, K. S. Wong and B. Z. Tang, Polym. Chem., 2010, 1, 426-429.

21 X. Wang, Y. Sun, S. Chen , X. Guo, M. Zhang, X. Li, Y. Li and H. Wang, Macromolecules, 2012, 45, 1208-1216.

22 B. Valeur, Molecular Fluorescence: Principle and Applications, Wiely-VCH, Weinheim, 2002. 


\section{Contribution to knowledge}

1. Demonstrated a phosgene-free synthetic route to isocyanates and polyurethanes.

2. Synthesized latent isocyanate terminated prepolymers.

3. Synthesized methacrylate copolymers with the pendent latent isocyanate groups.

4. Demonstrated the fluorescence detection of trace amounts of isocyanates and explosives in air and in solution by fluorescent conjugated polymers. 


\section{Publications}

1. Ghosh, K. R.; Saha, S.; Wang, Z. Y. "Ultra-sensitive detection of explosives in solution and film as well as the development of thicker film effectiveness by tetraphenylethene moiety in AIE active fluorescent conjugated polymer" Polym. Chem. 2014, 5, 5638.

2. Ghosh, K. R.; Saha, S.; Gao, J. P.; Wang, Z. Y. "Direct detection of ultralow trace amounts of isocyanates in air using a fluorescent conjugated polymer" Chem. Commun. 2014, 50, 176.

3. Saha, S.; Ghosh, K. R.; Gao, J. P.; Wang, Z. Y. "Highly sensitive dual-mode fluorescence detection of lead ion in water using aggregation-induced emissive polymers" Macromol. rapid commun. 2014, 35, 1592.

4. Saha, S.; Ghosh, K. R.; Hao, W.; Gao, J. P.; Wang, Z. Y.; Ma, J.; Chiniforooshan, Y.; Bock, W. J. "Highly sensitive and selective fluorescence turn-on detection of lead Ion in water using fluorene-based compound and polymer” J. Matter. Chem A. 2014, 2, 5024.

\section{Seminar and Conference Presentations}

1. Ghosh, K. R.; Saha, S.; Gao, J. P.; Wang, Z. Y. "Ultra Low Detection of Isocyanates and Nitro-Explosives Using Aggregation-Induced Emission Active Fluorescent Conjugated Polymer" Poster presentation, Ottawa-Carleton Chemistry Institute Conference day, Carleton University, Ottawa, ON, May 2014.

2. Saha, S.; Ghosh, K. R.; Gao, J. P.; Wang, Z. Y. "Amplified Fluorescence Detection of Isocyanates, Nitro-Explosives and Toxic Metals Using Aggregation-Induced Emissive Polymers" Oral presentation, Ottawa-Carleton Chemistry Institute Conference day, Carleton University, Ottawa, ON, May 2014. 


\section{Appendix A: Spectra of compounds in Chapter 2}

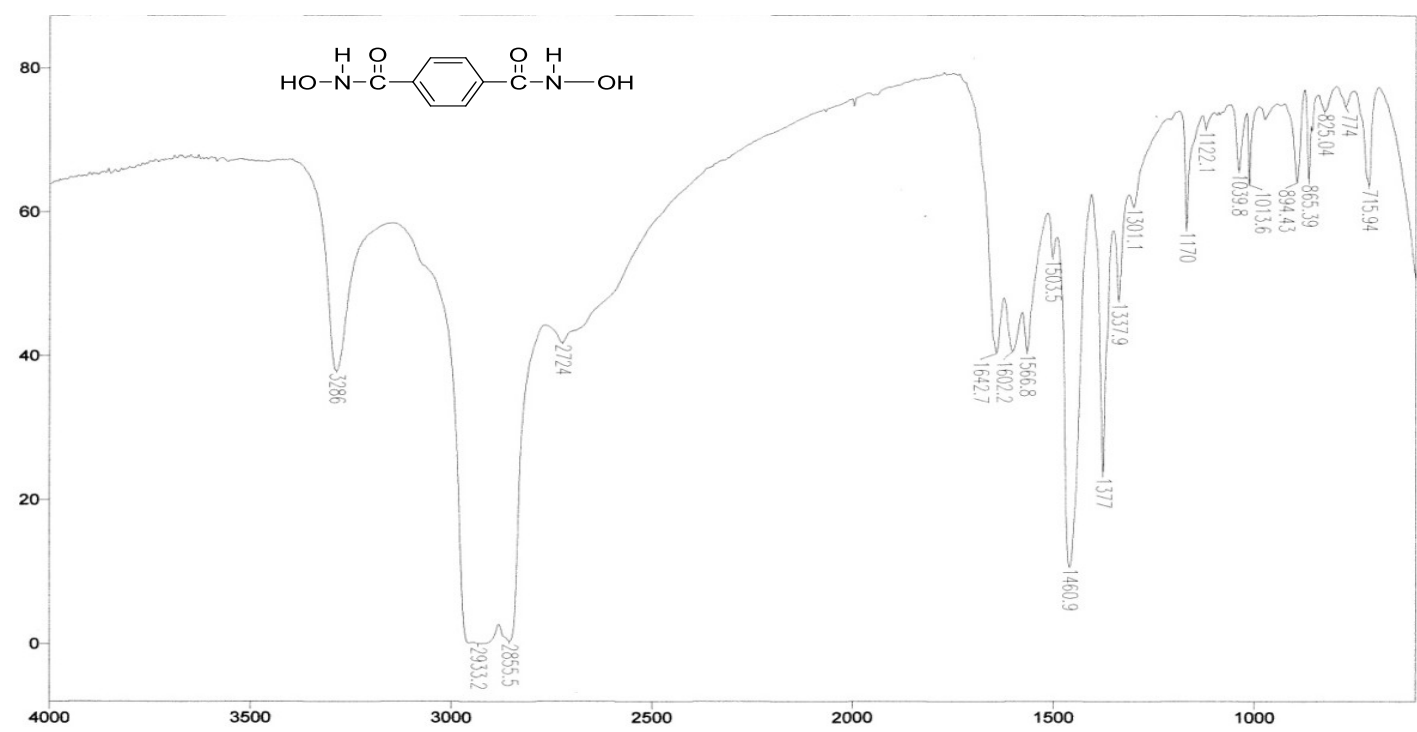

Figure S2.1. IR spectrum of $N 1$, N4-dihydroxyterephthalamide ( $\mathrm{NaCl}$ plate)

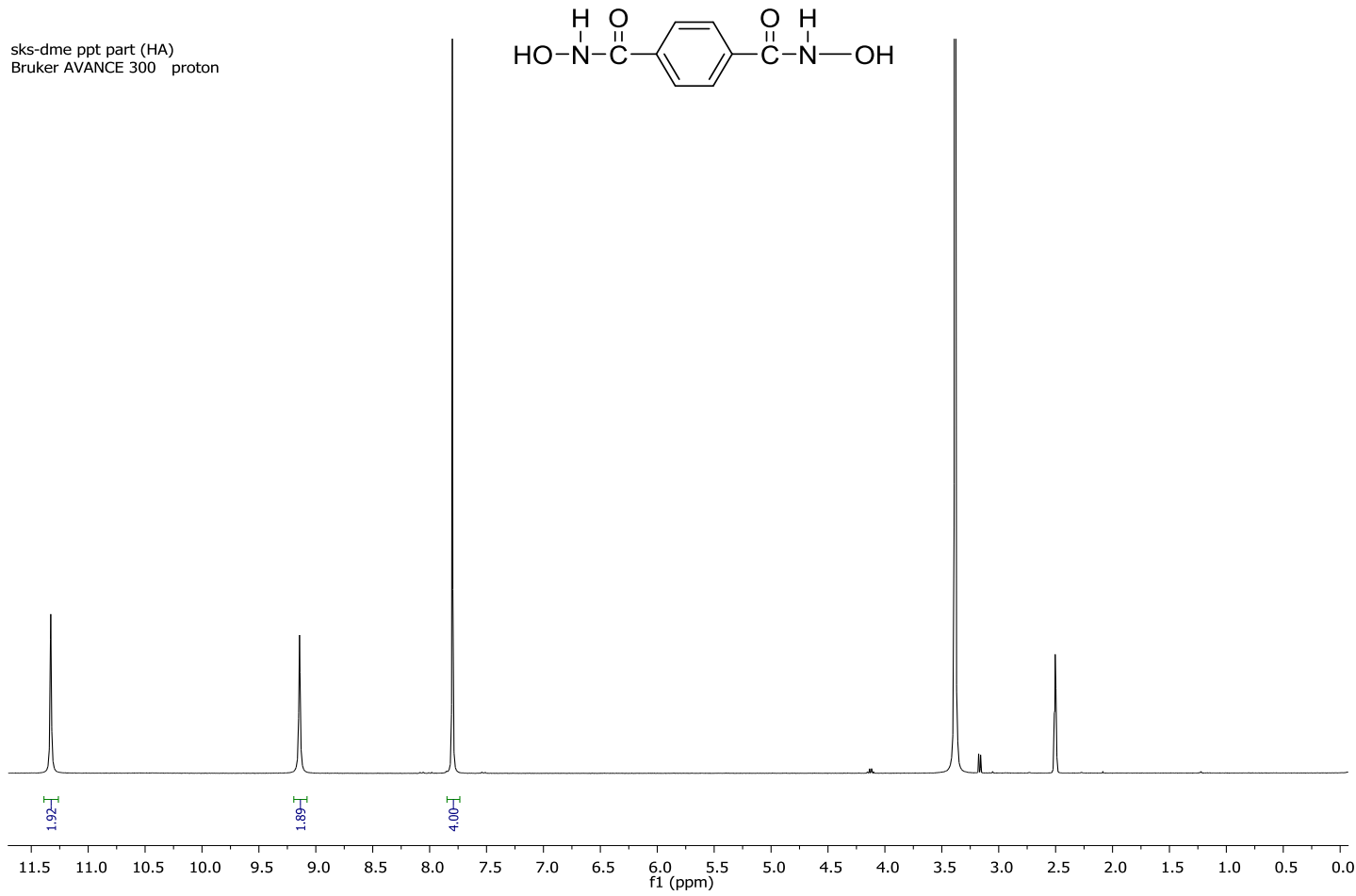

Figure S2.2. The ${ }^{1} \mathrm{H}$ NMR spectrum (300 MHz, DMSO- $\mathrm{d}_{6}$ ) of N1, N4-dihydroxyterephthalamide 


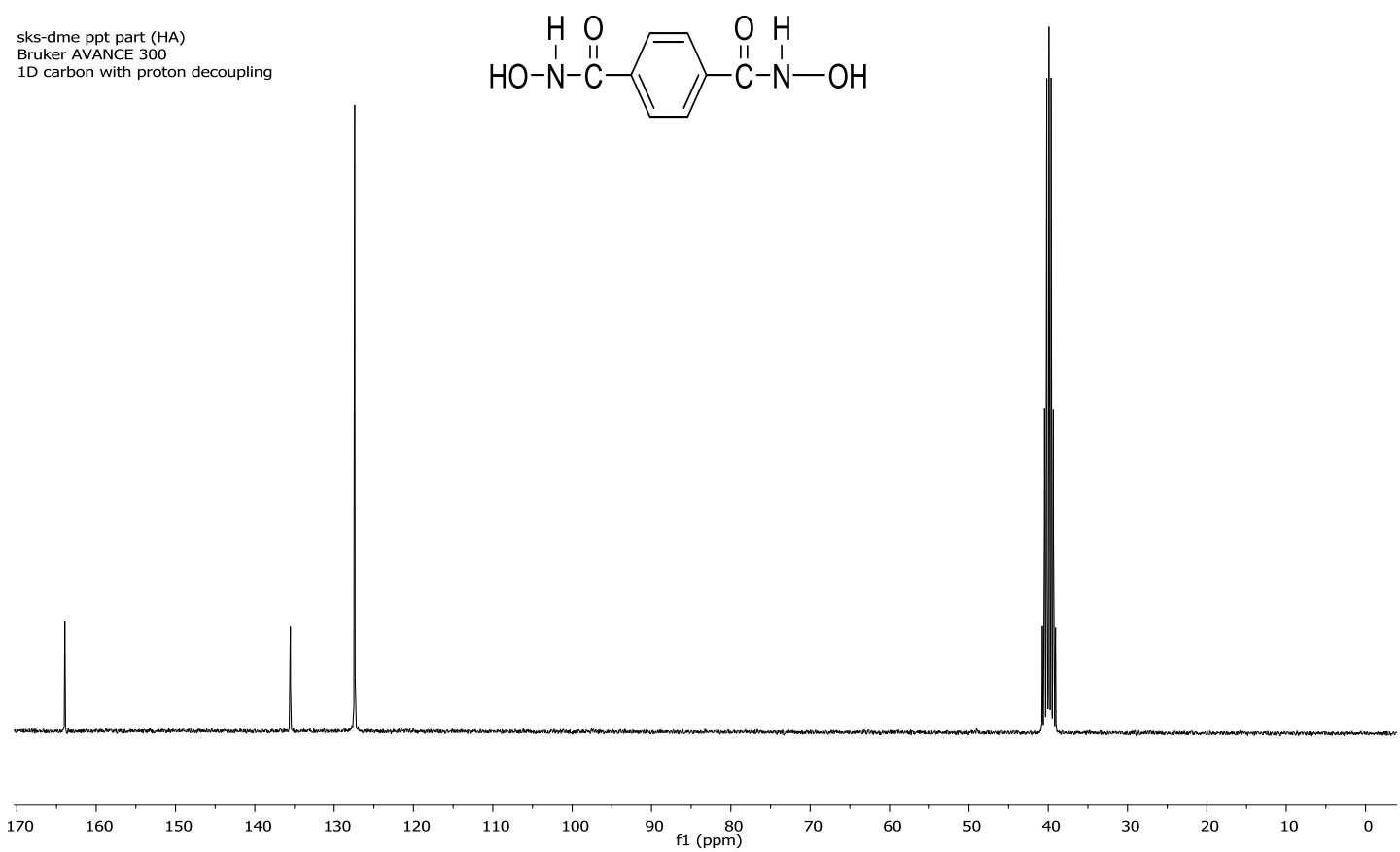

Figure S2.3. The ${ }^{13} \mathrm{C}$ NMR spectrum (75 MHz, DMSO-d ${ }_{6}$ ) of $N 1$, N4-dihydroxyterephthalamide 2 d1799 Scan $7 \quad \mathrm{RT}=1: 48 \quad 1008=734899 \mathrm{mv} 18$-Oct-2016 $13: 52$ HRP +EI petro 0283

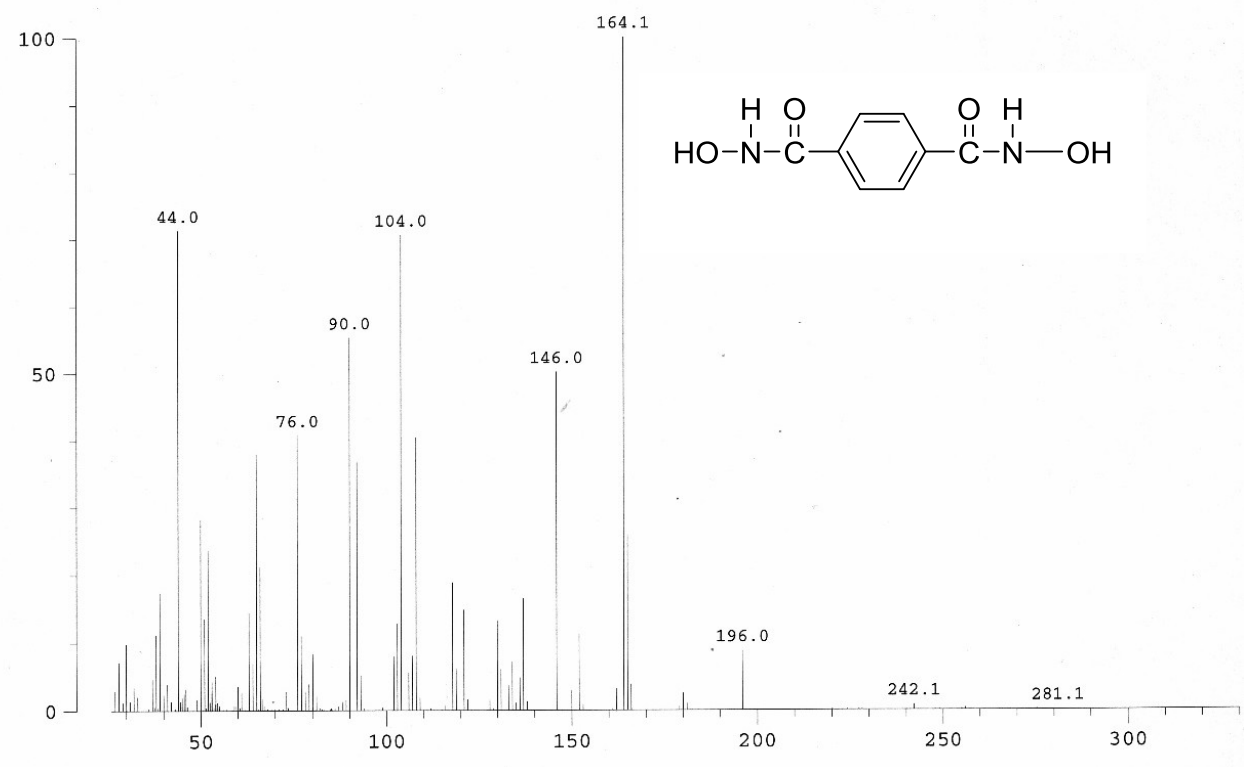

Figure S2.4. EI mass spectrum of N1, N4-dihydroxyterephthalamide 


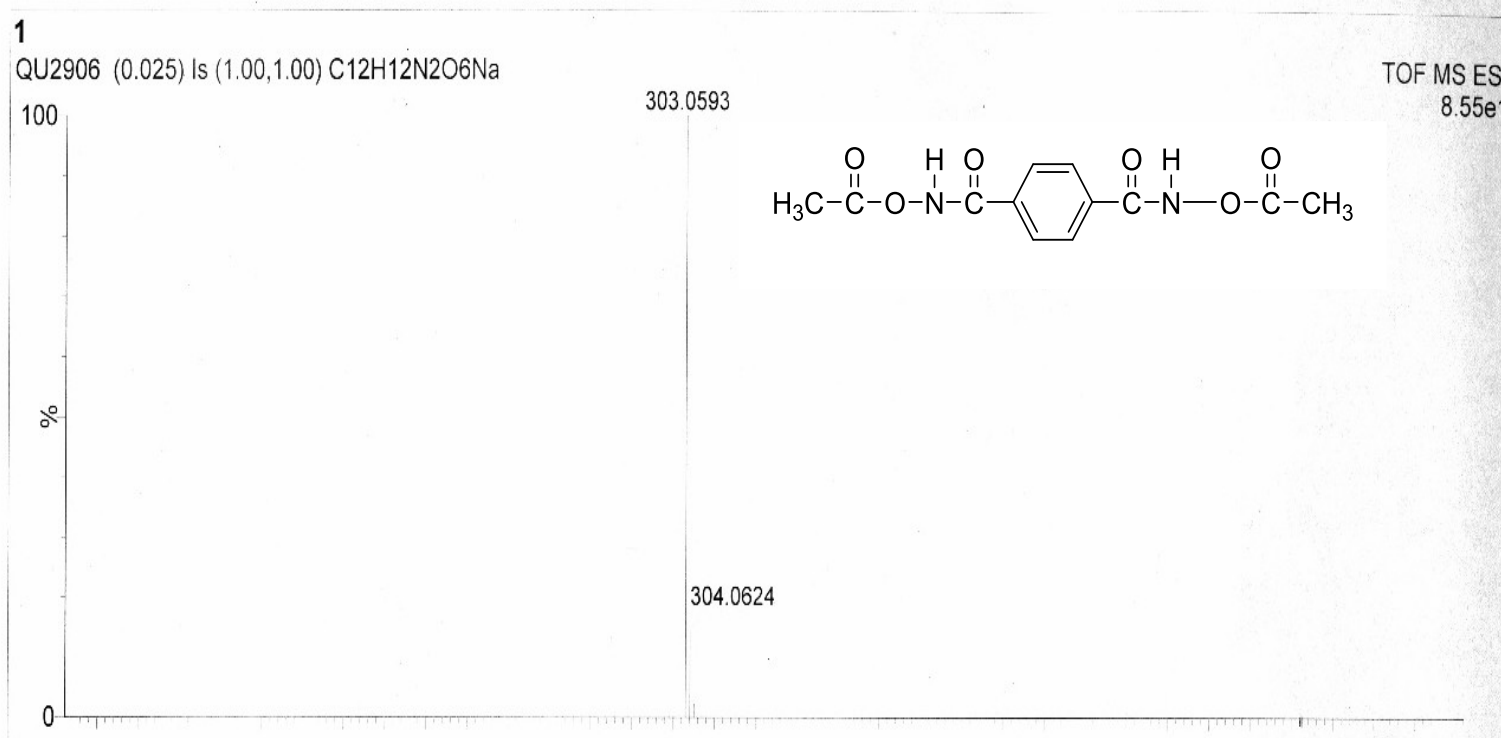

Figure S2.5. ESI mass spectrum of N1, N4-diacetoxyterephthalamide

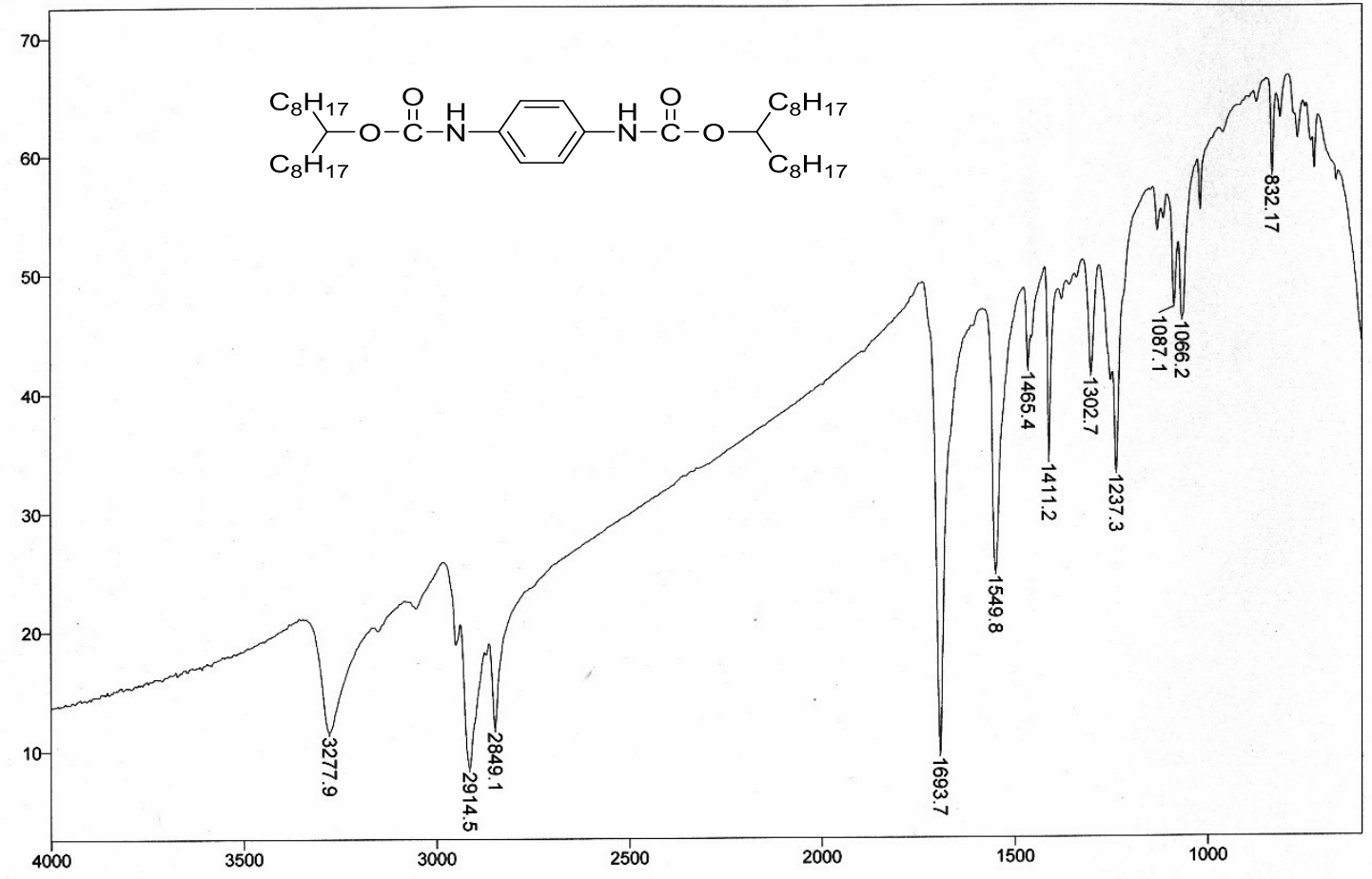

Figure S2.6. IR spectrum of di(heptadecan-9-yl) 1,4-phenylenedicarbamate (compound 1) ( $\mathrm{NaCl}$ plate) -in absence of base catalyst 


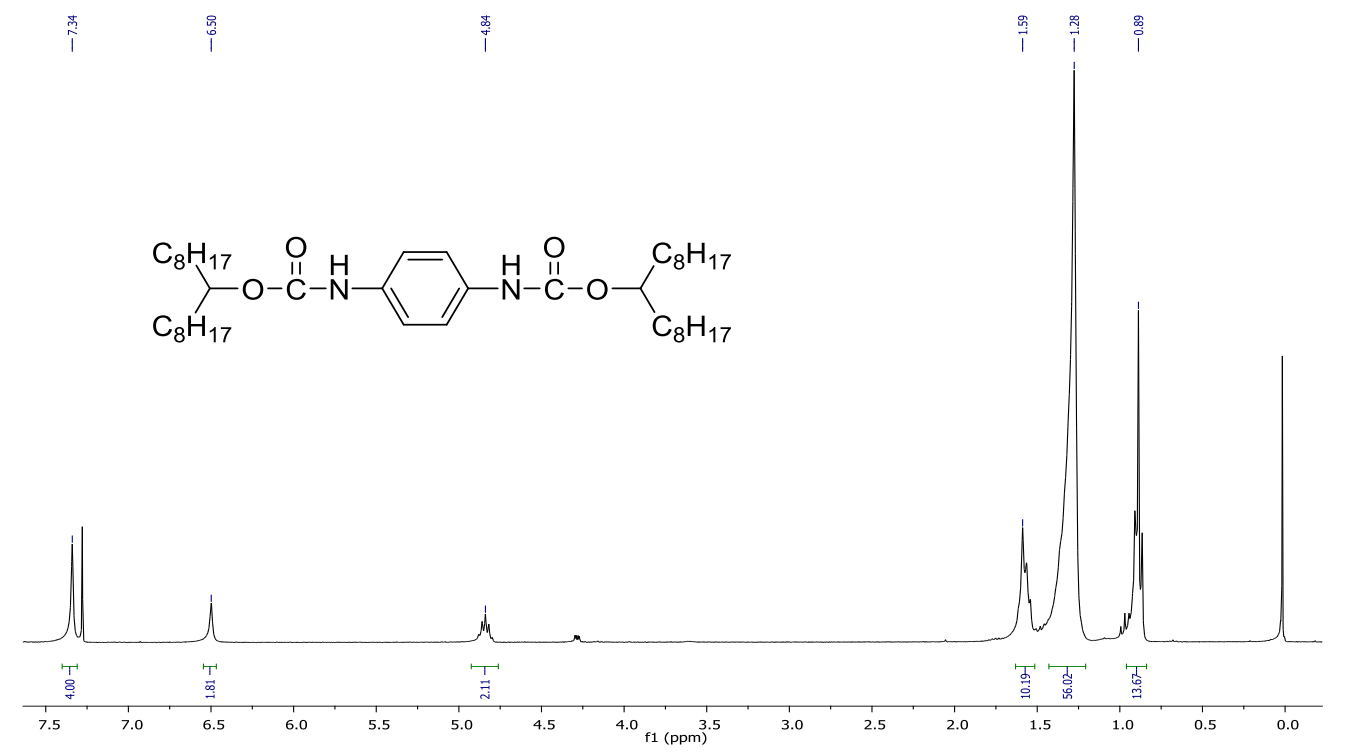

Figure S2.7. The ${ }^{1} \mathrm{H}$ NMR spectrum (300 MHz, $\mathrm{CDCl}_{3}$ ) of di(heptadecan-9-yl) 1,4 phenylenedicarbamate (compound $\mathbf{1}$ ) -in absence of base catalyst

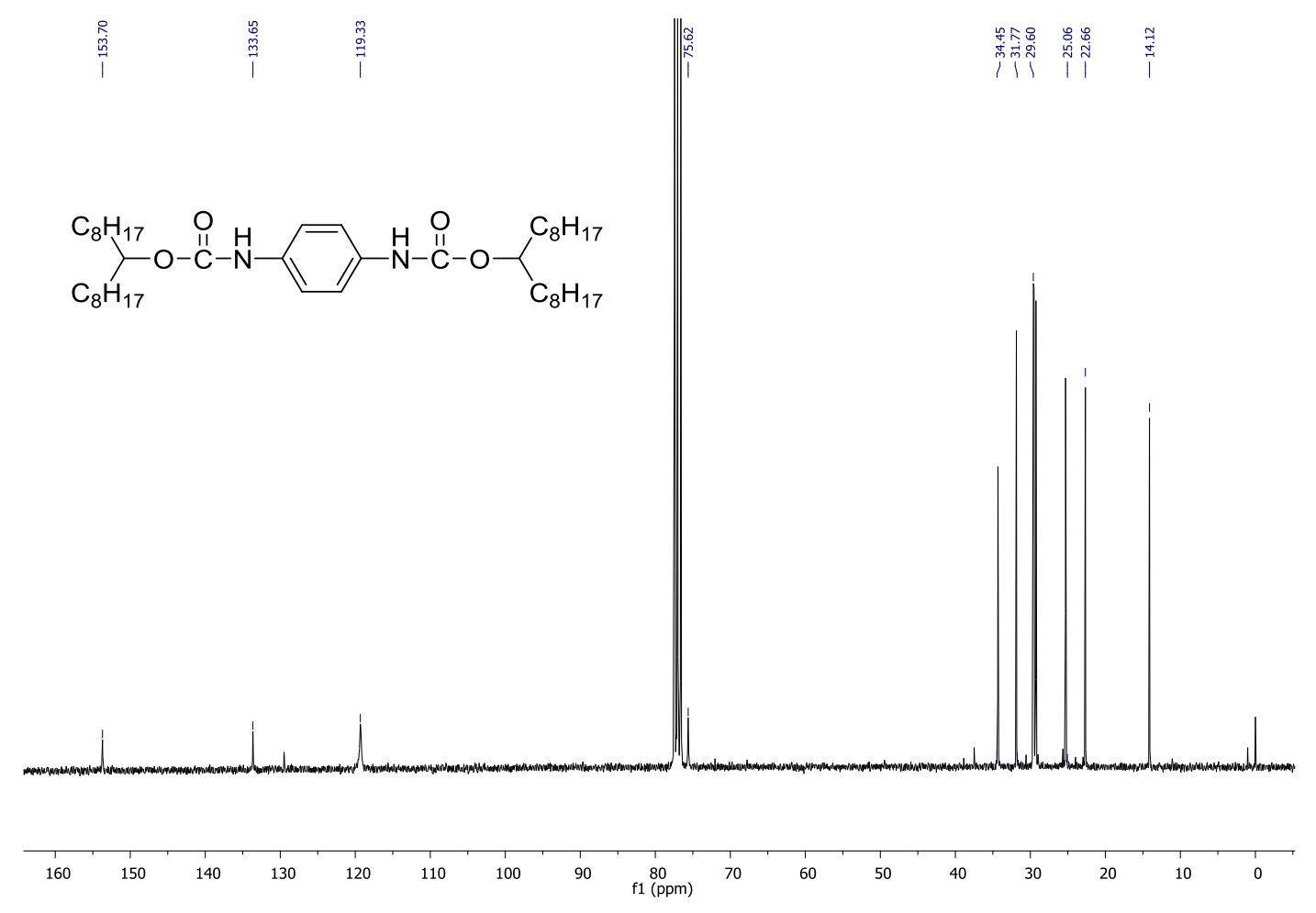

Figure S2.8. The ${ }^{13} \mathrm{C}$ NMR spectrum (75 MHz, $\mathrm{CDCl}_{3}$ ) of di(heptadecan-9-yl) 1,4 phenylenedicarbamate (compound $\mathbf{1}$ ) -in absence of base catalyst 


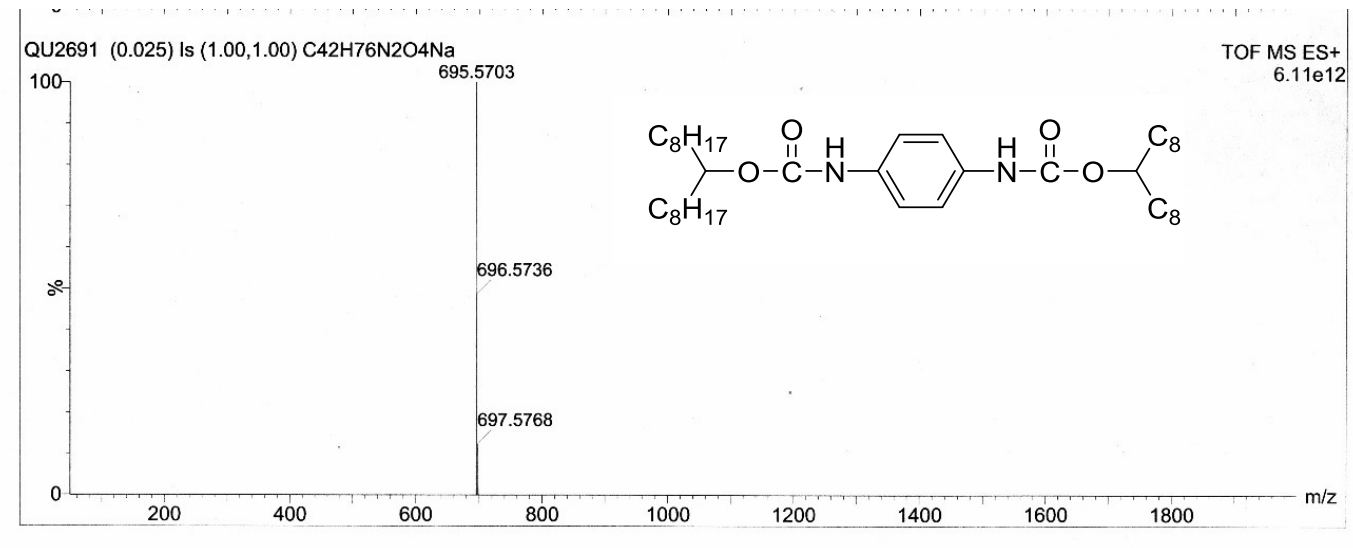

Figure S2.9. ESI Mass spectrum of di(heptadecan-9-yl) 1,4-phenylenedicarbamate (compound 1) - in absence of base catalyst

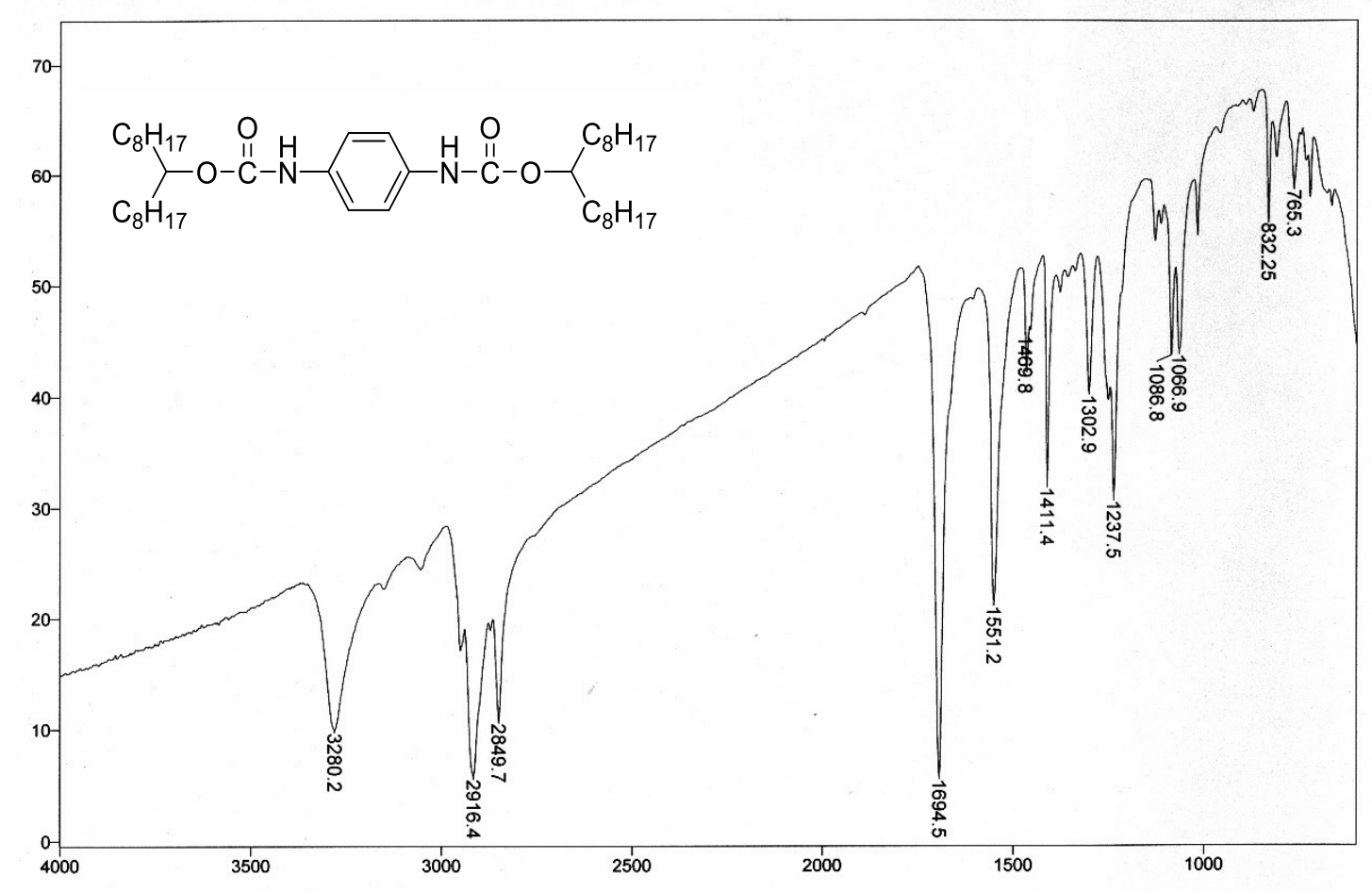

Figure S2.10. IR spectrum of di(heptadecan-9-yl) 1,4-phenylenedicarbamate (compound 1) $(\mathrm{NaCl}$ plate $)$-in the presence of base catalyst 


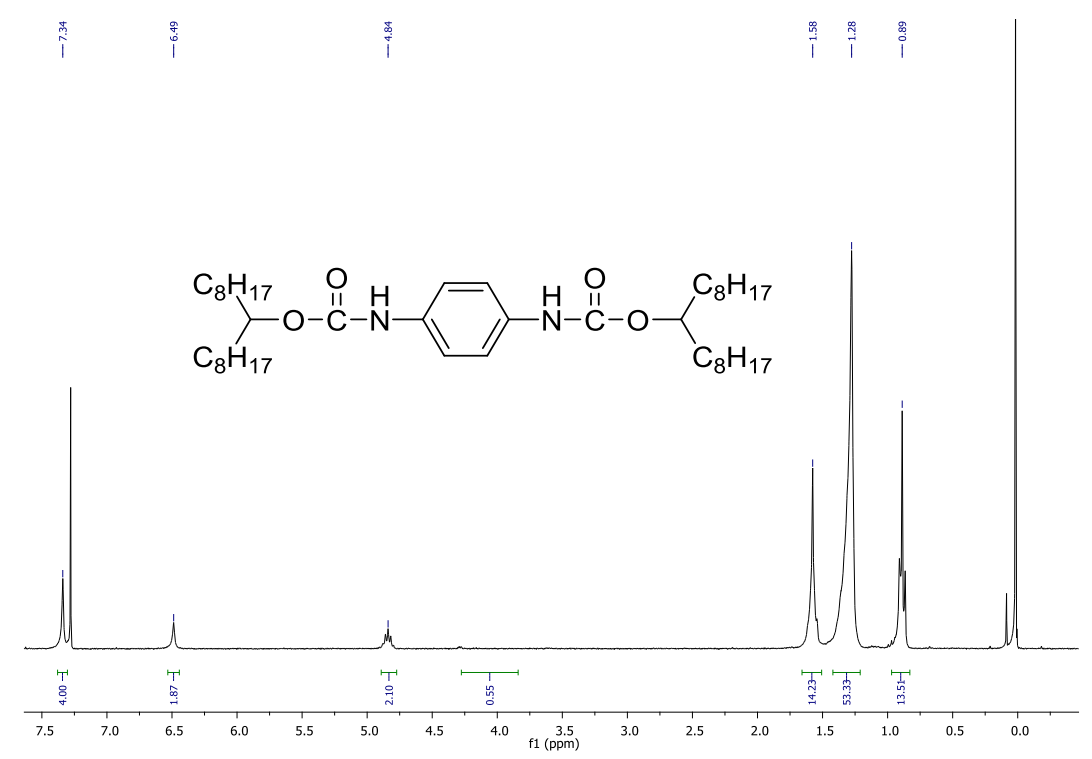

Figure S2.11. The ${ }^{1} \mathrm{H}$ NMR spectrum $\left(300 \mathrm{MHz}, \mathrm{CDCl}_{3}\right.$ ) of di(heptadecan-9-yl) 1,4phenylenedicarbamate (compound 1) -in the presence of base catalyst

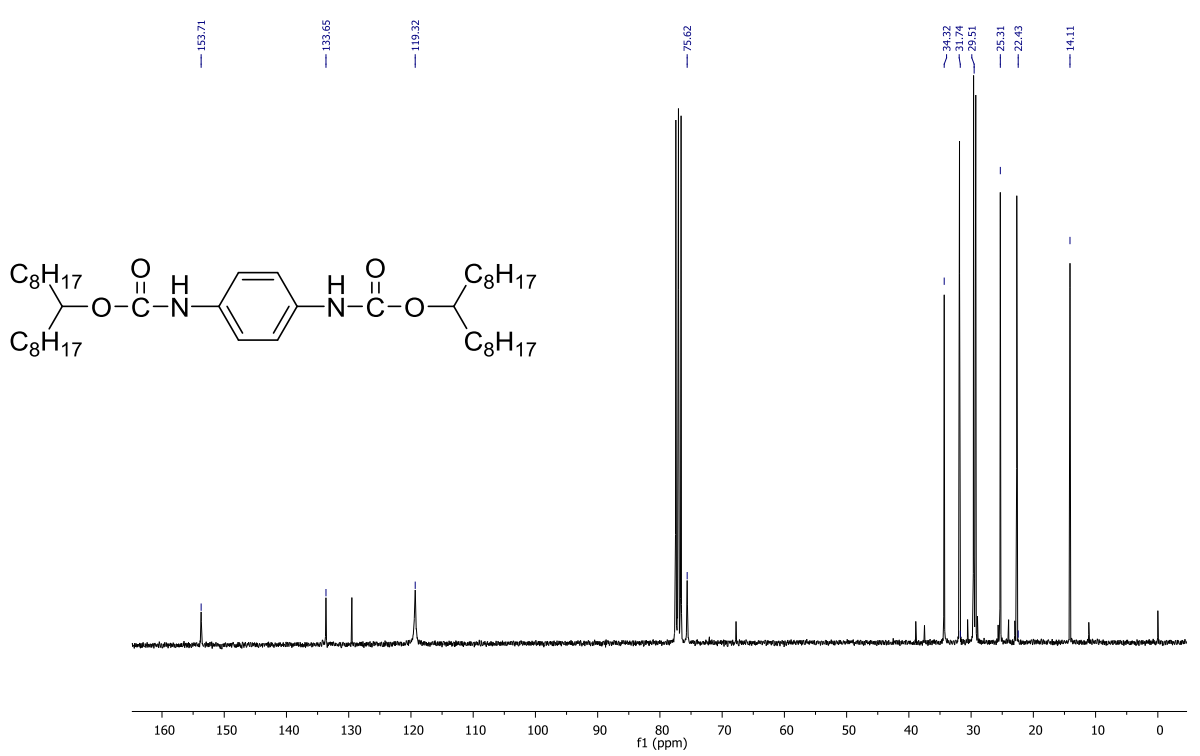

Figure S2.12. The ${ }^{13} \mathrm{C}$ NMR spectrum (75 MHz, $\mathrm{CDCl}_{3}$ ) of di(heptadecan-9-yl) 1,4phenylenedicarbamate (compound 1) -in the presence of base catalyst 


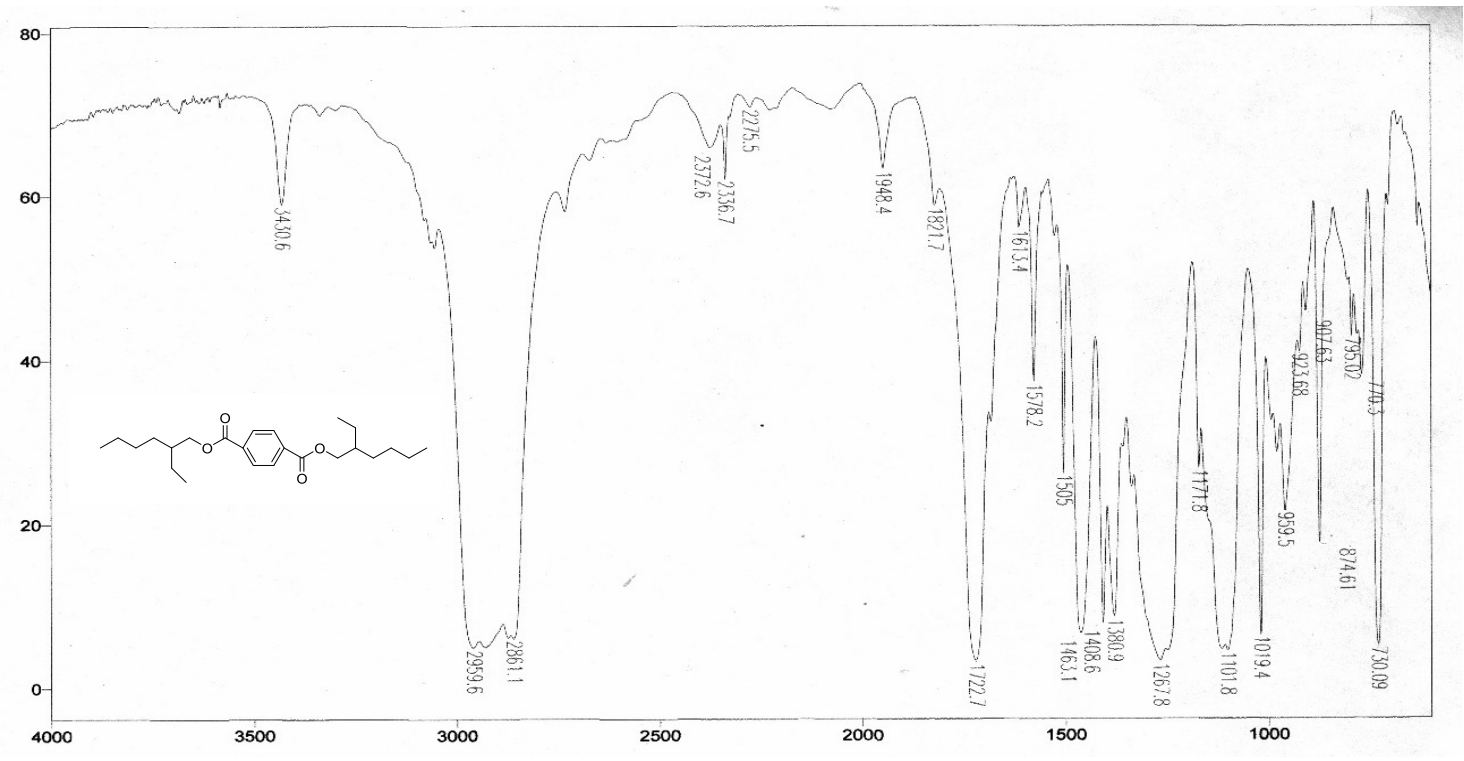

Figure S2.13. IR spectrum after 5 min bubbled of $\mathrm{CO}_{2}$ into Dioctyl terephthalate at room temperature $(\mathrm{NaCl}$ plate)

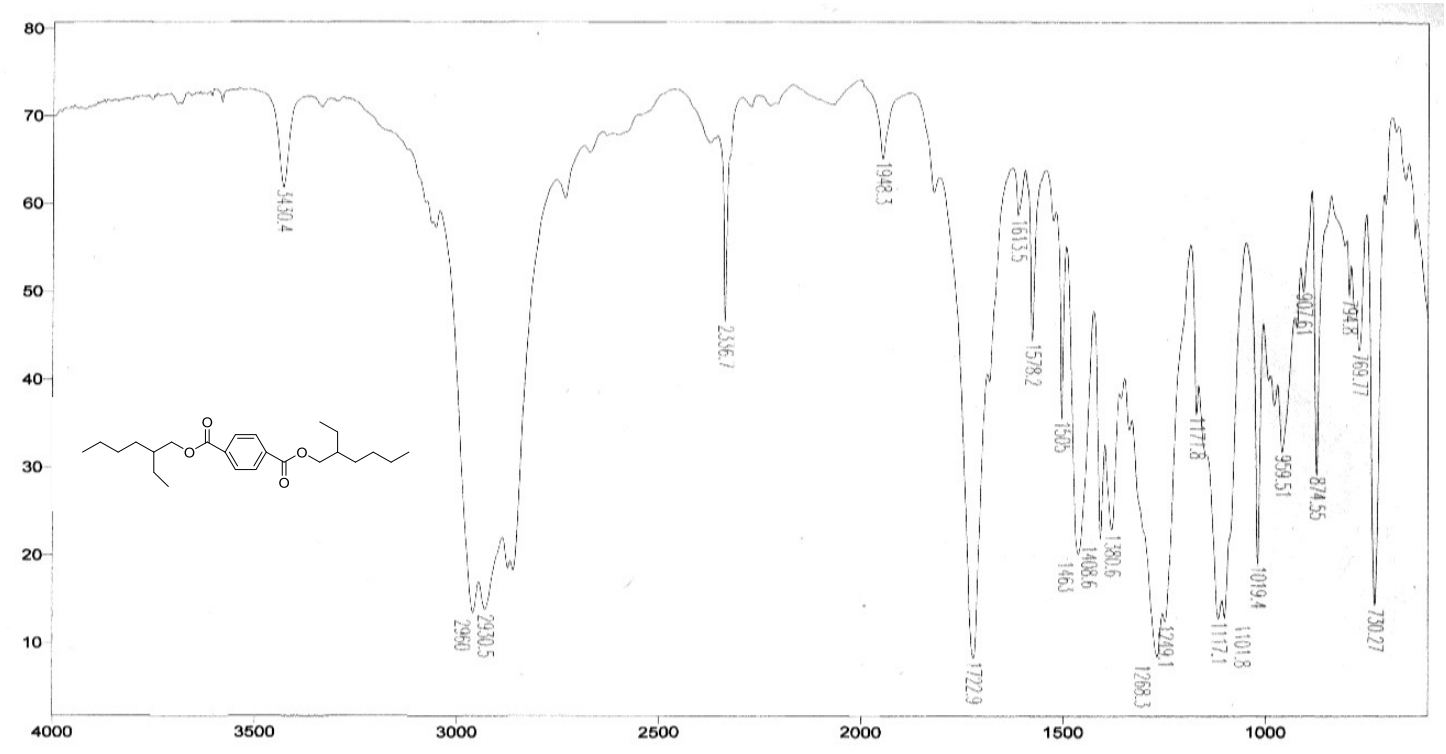

Figure S2.14 IR spectrum after 10 minutes bubbled of $\mathrm{CO}_{2}$ into Dioctyl terephthalate at room temperature $(\mathrm{NaCl}$ plate) 


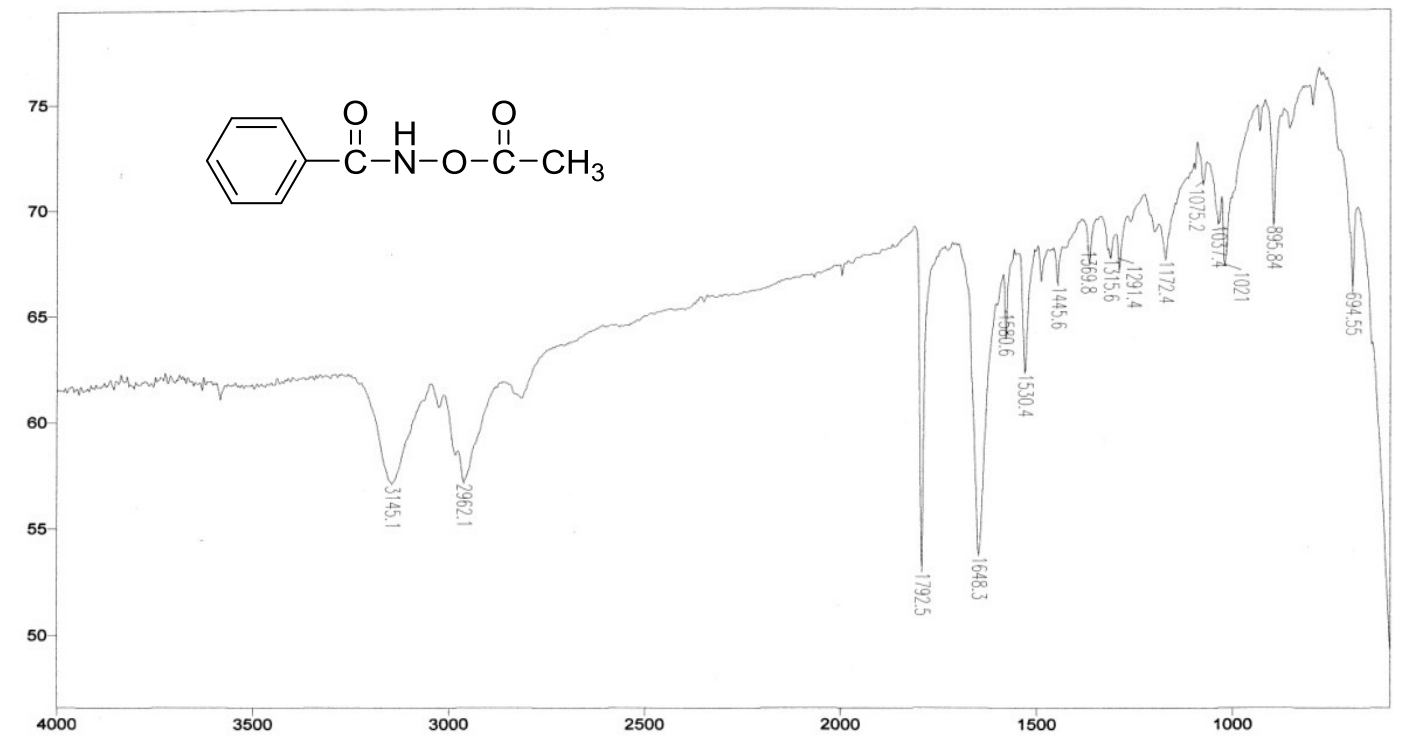

Figure S2.15. IR of $\mathrm{N}$-acetoxybenzamide ( $\mathrm{NaCl}$ plate)

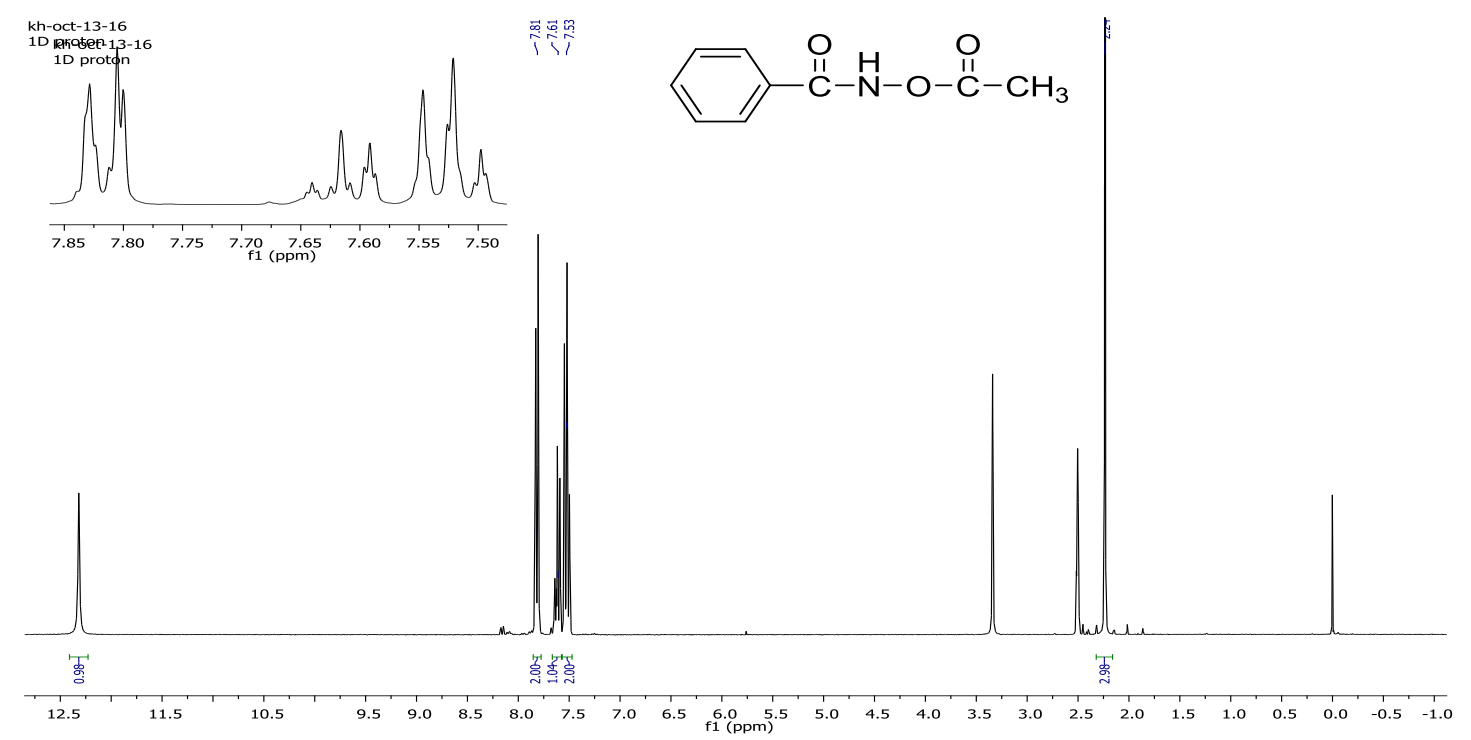

Figure S2.16. The ${ }^{1} \mathrm{H}$ NMR spectrum (300 MHz, DMSO- $\mathrm{d}_{6}$ ) of $N$-acetoxybenzamide 


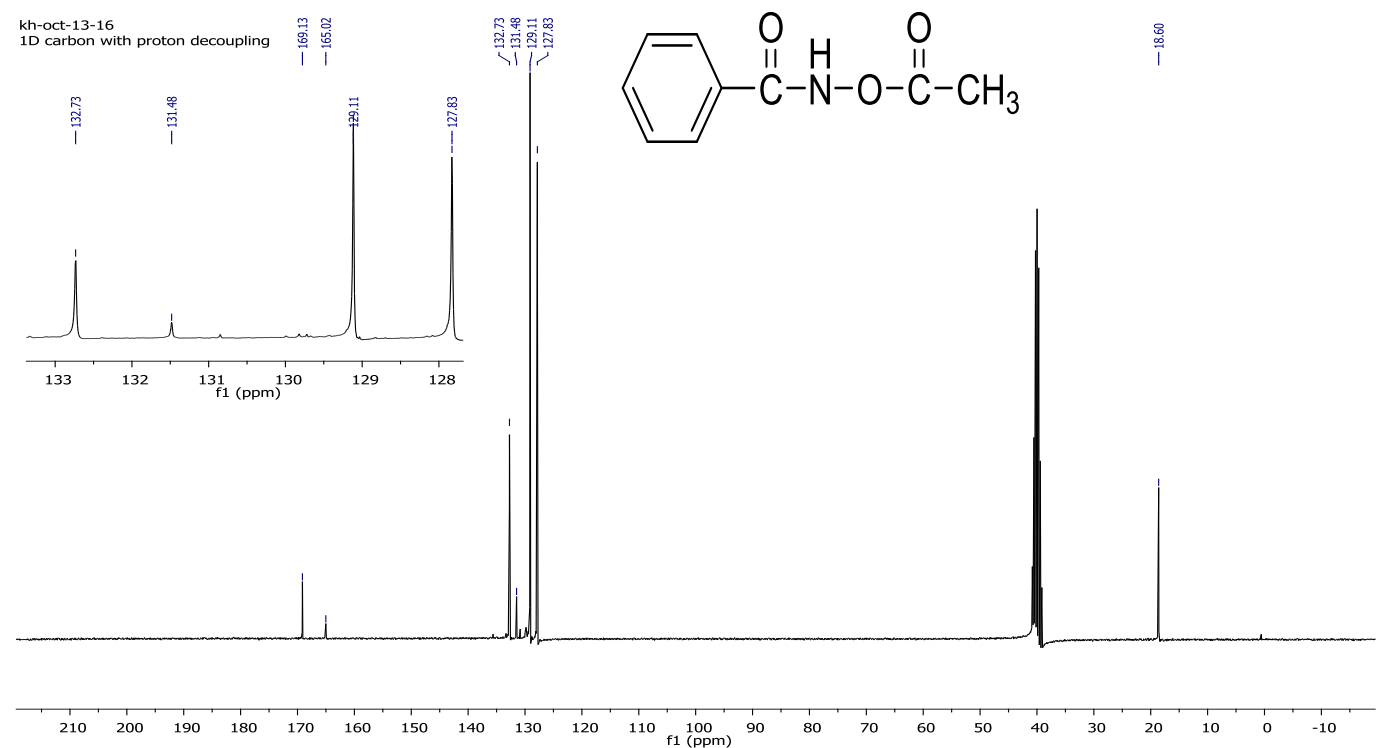

Figure S2.17. The ${ }^{13} \mathrm{C}$ NMR spectrum $\left(75 \mathrm{MHz}, \mathrm{DMSO}-\mathrm{d}_{6}\right)$ of $N$-acetoxybenzamide

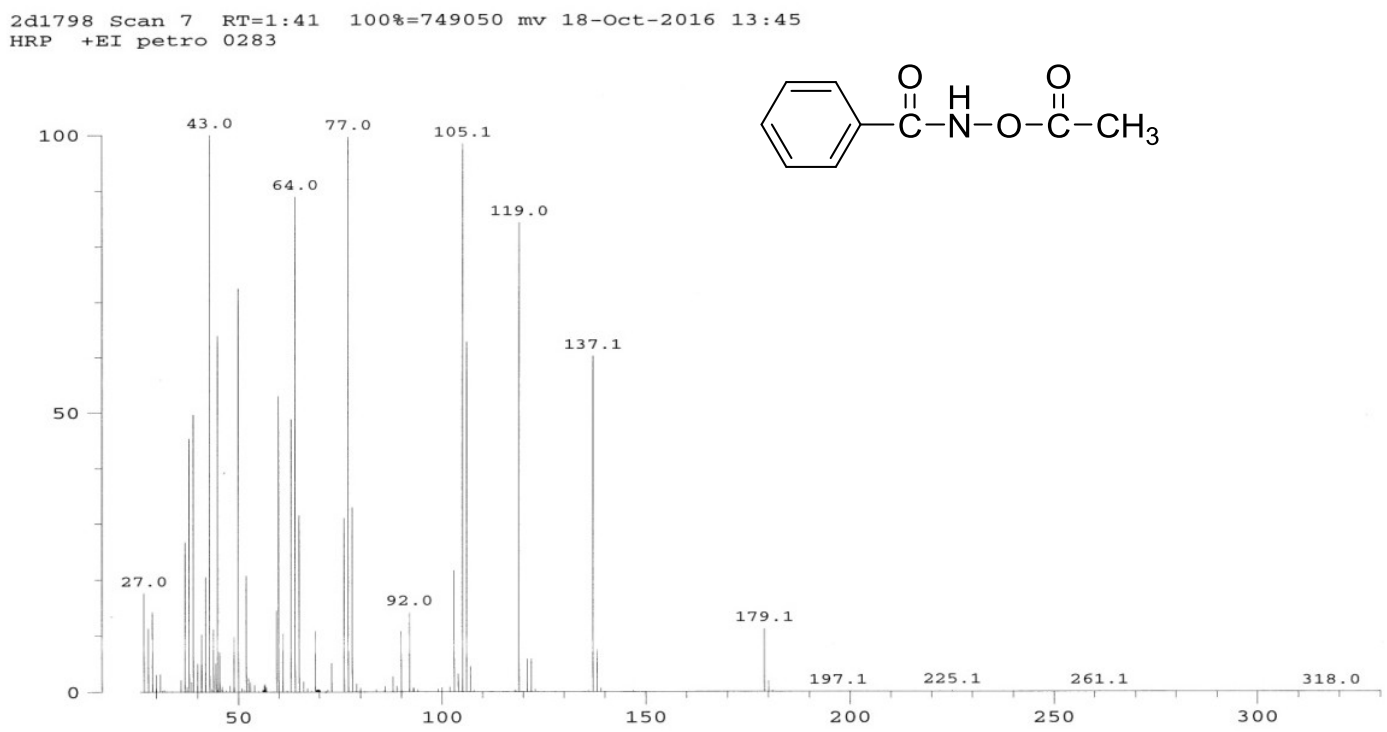

Figure S2.18. EI mass spectrum of $N$-acetoxybenzamide 


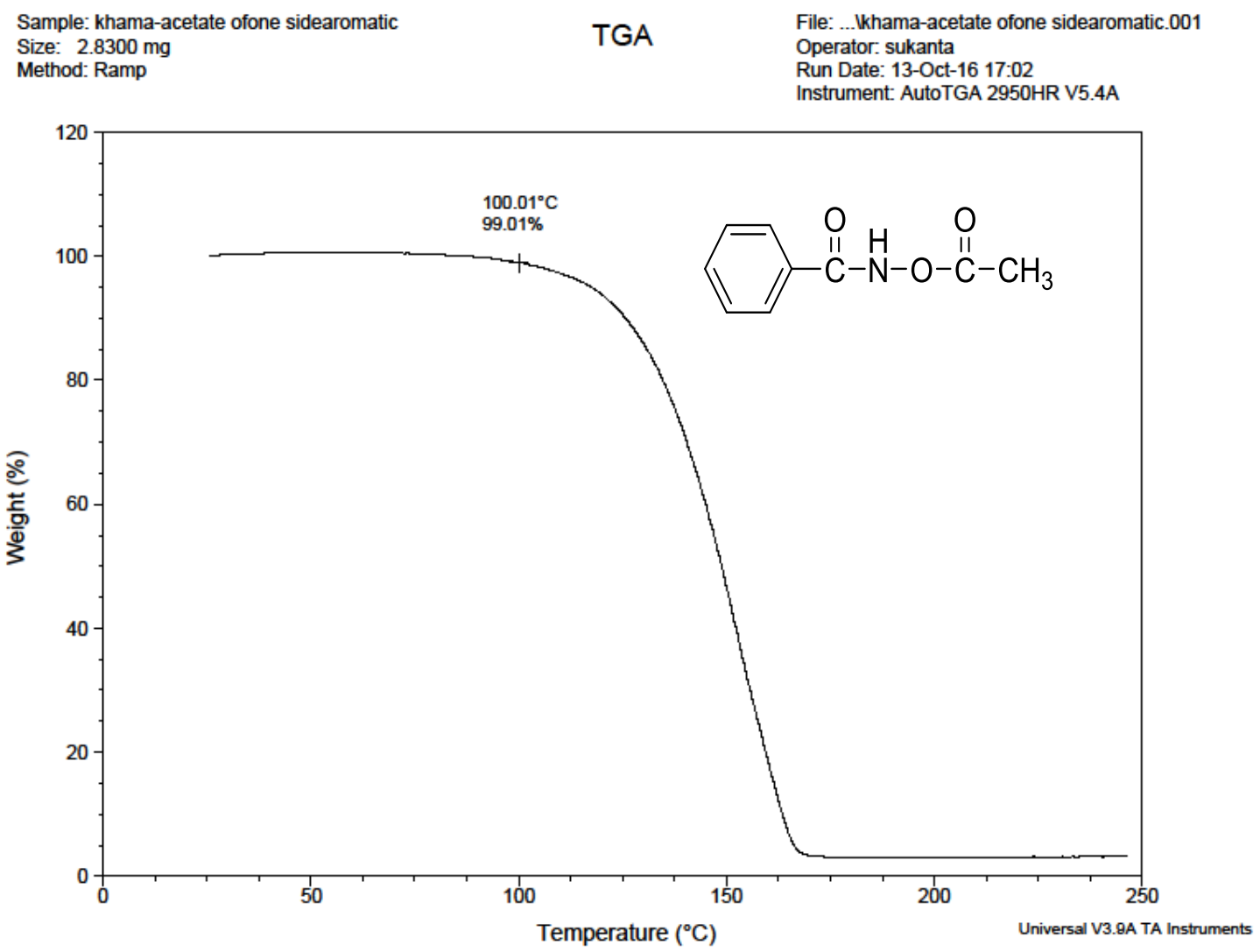

Figure S2.19. TGA of $N$-acetoxybenzamide

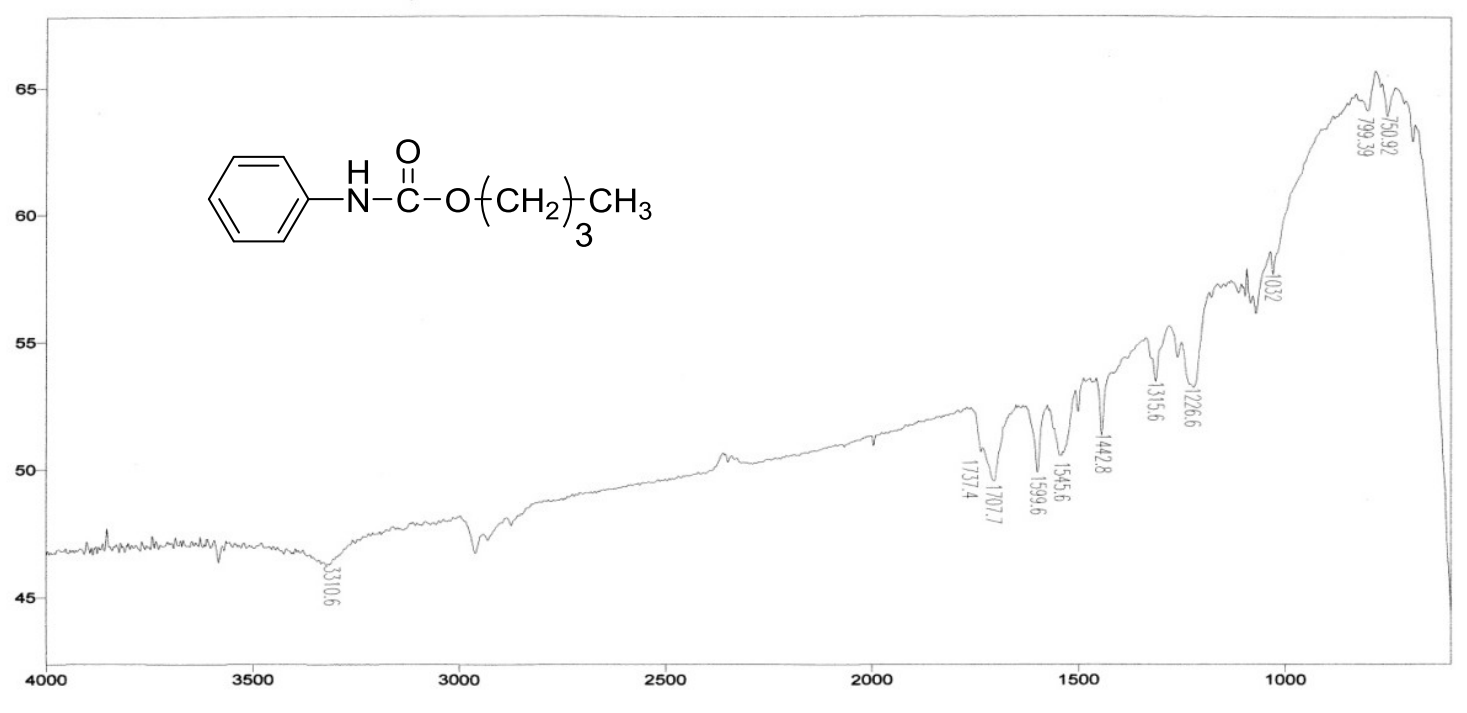

Figure S2.20. IR of butyl phenylcarbamate (compound 2) ( $\mathrm{NaCl}$ plate) 

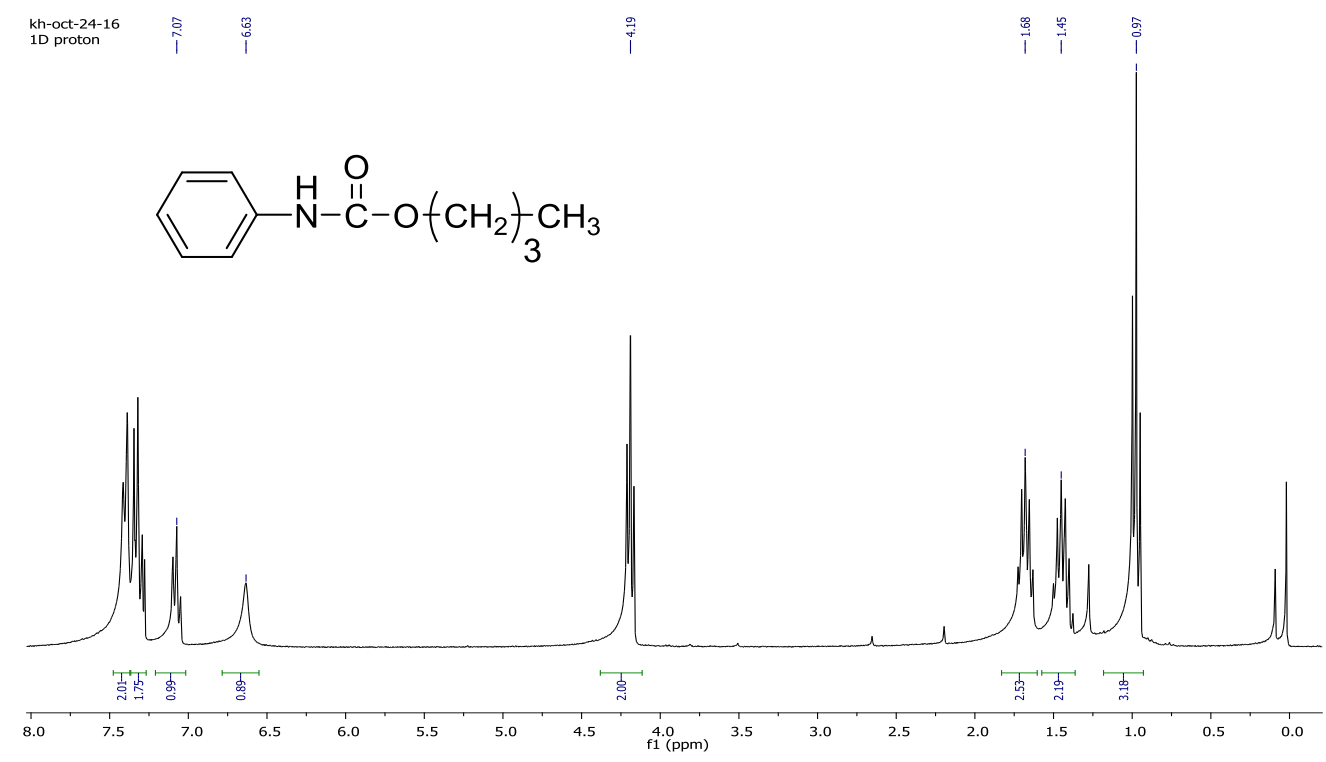

Figure S2.21. The ${ }^{1} \mathrm{H}$ NMR spectrum $\left(300 \mathrm{MHz}, \mathrm{CDCl}_{3}\right.$ ) of butyl phenylcarbamate (compound 7)

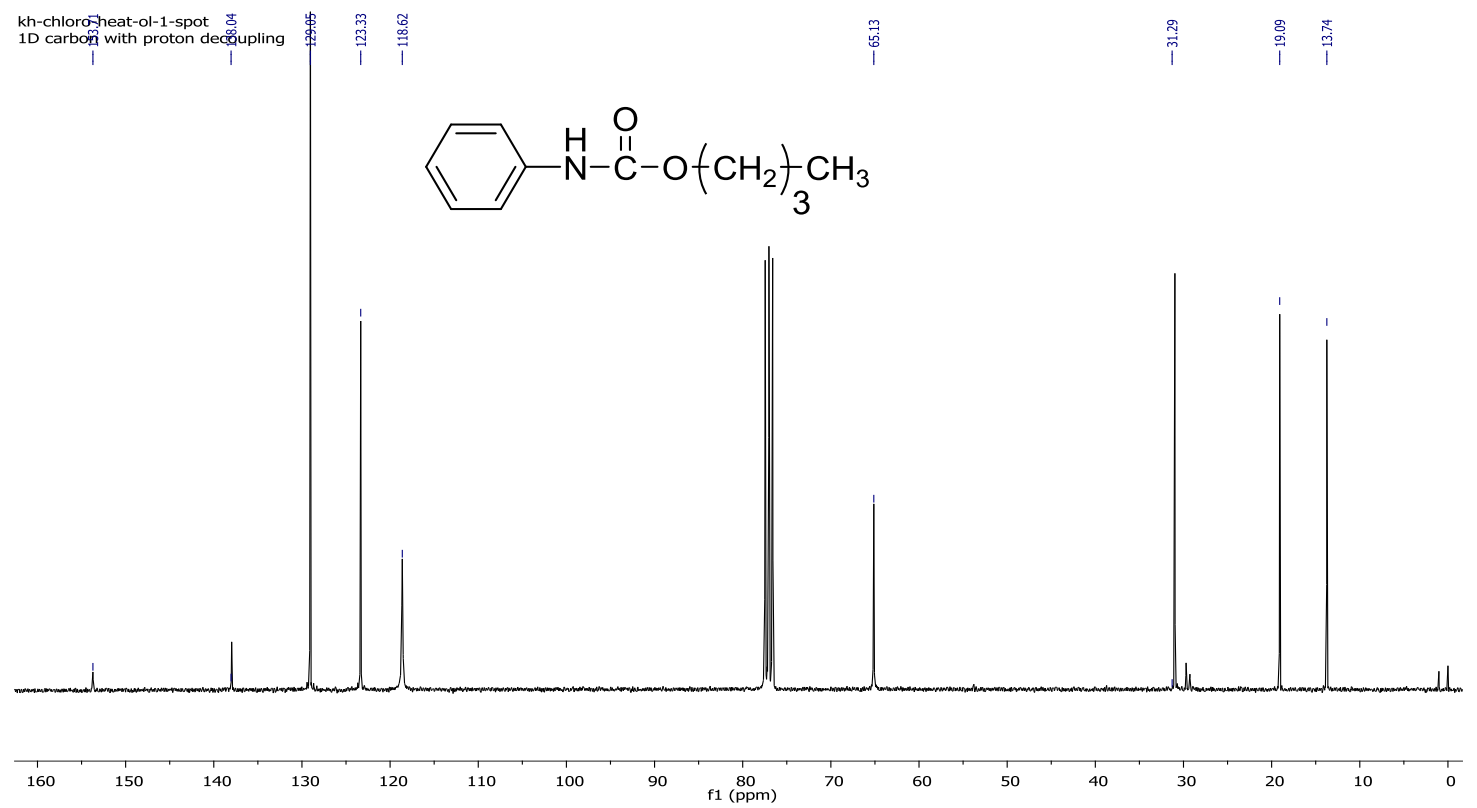

Figure S2.22. The ${ }^{13} \mathrm{C}$ NMR spectrum $\left(75 \mathrm{MHz}, \mathrm{CDCl}_{3}\right.$ ) of butyl phenylcarbamate (compound 7) 


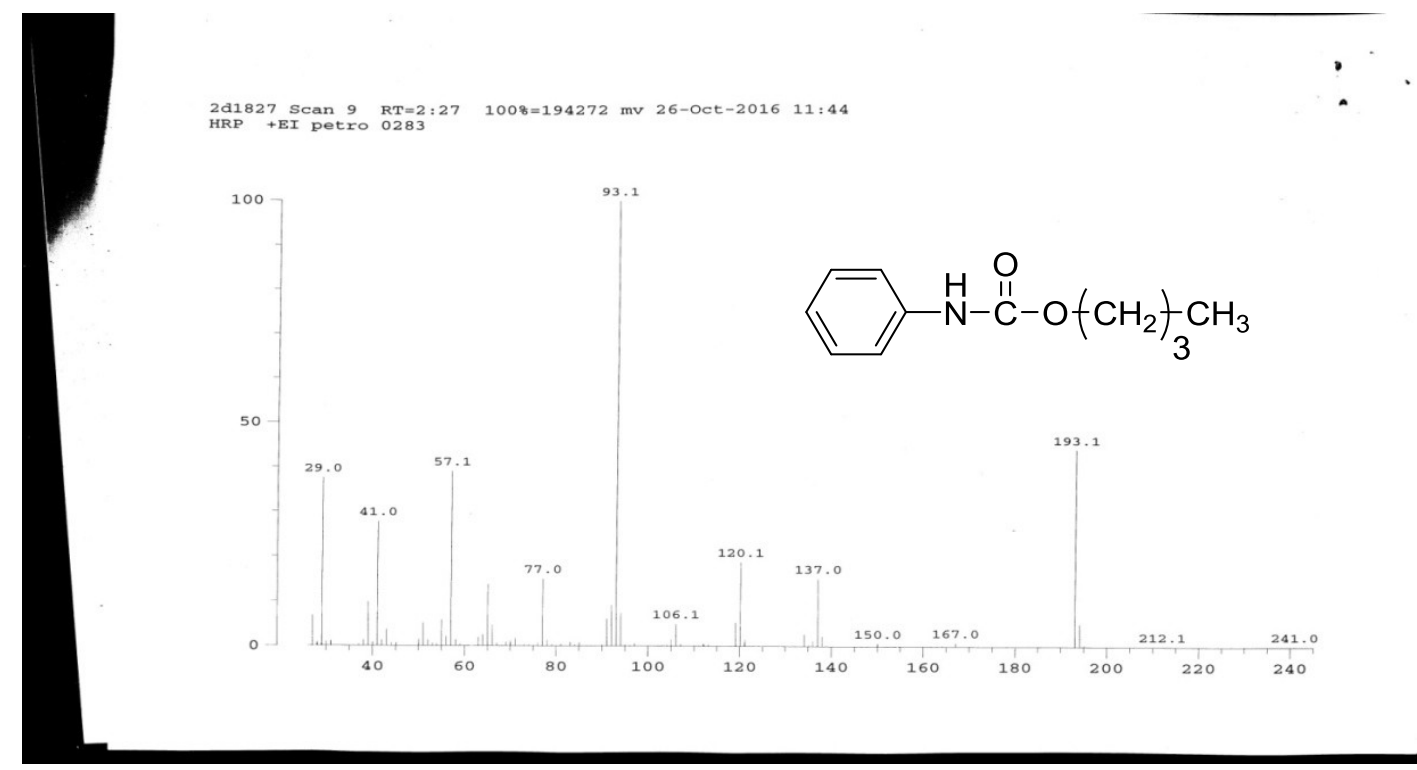

Figure S2.23. EI mass spectrum of butyl phenylcarbamate (compound 7)

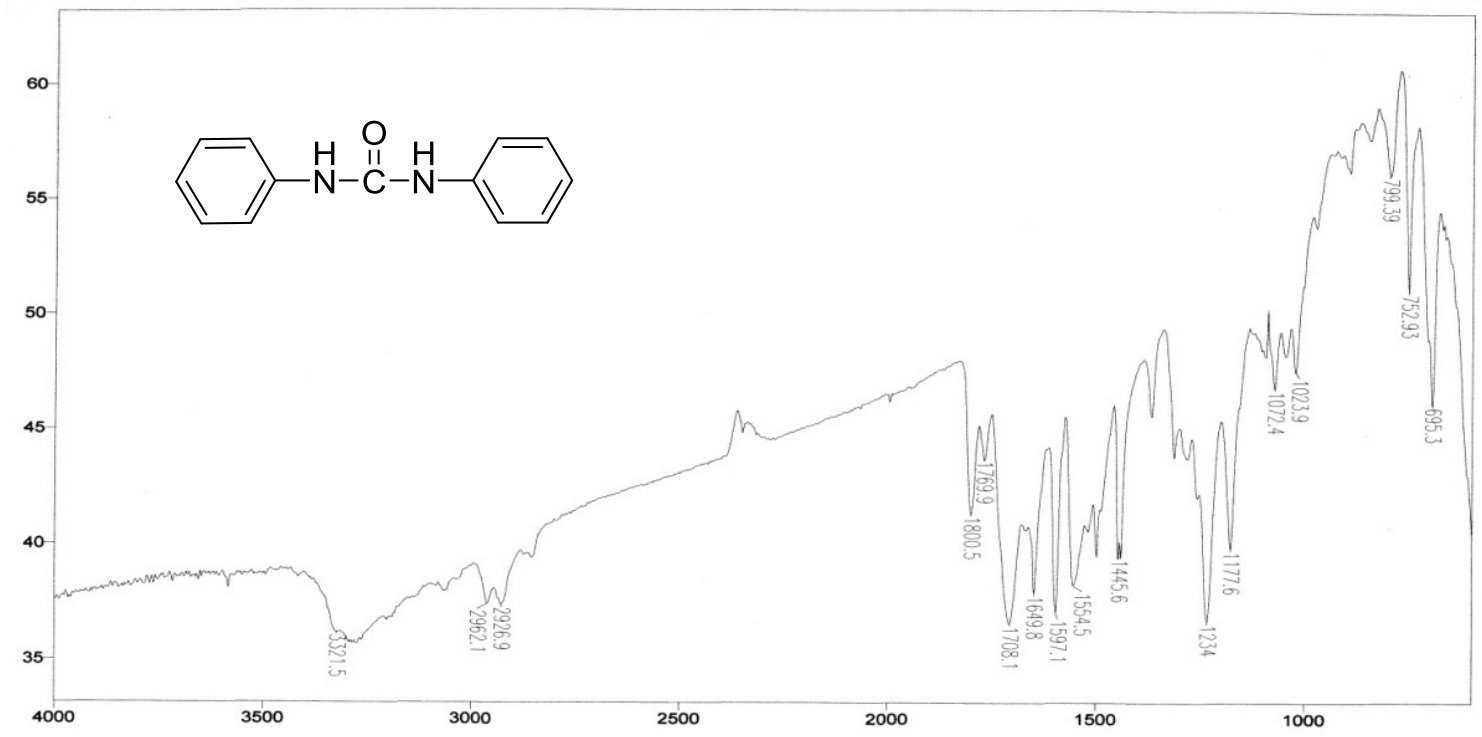

Figure S2.24. IR of 1,3-diphenylurea (compound 3) ( $\mathrm{NaCl}$ plate) 


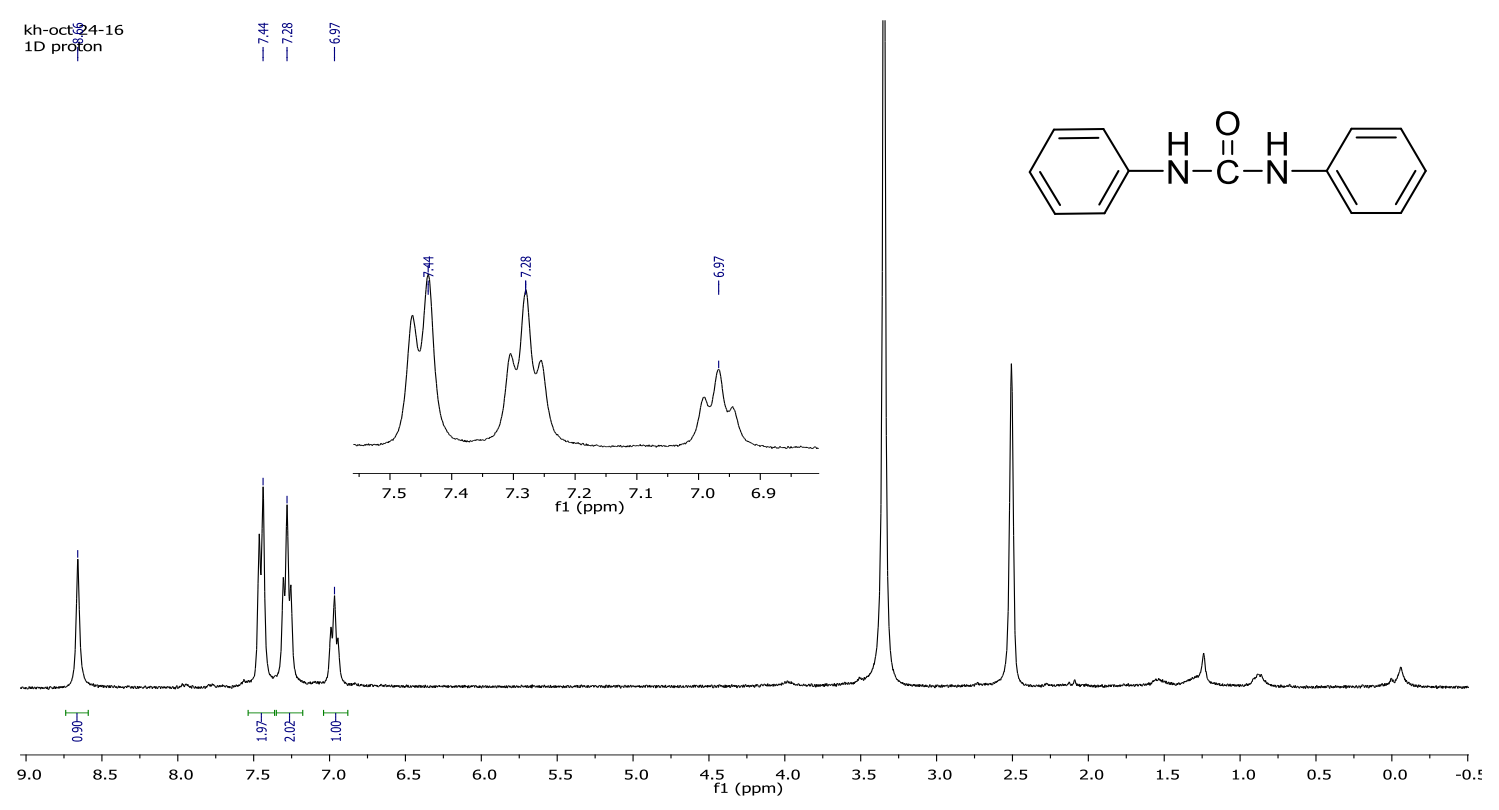

Figure S2.25.The ${ }^{1} \mathrm{H}$ NMR spectrum (300 MHz, DMSO-d ${ }_{6}$ ) of 1,3-diphenylurea (compound 3)

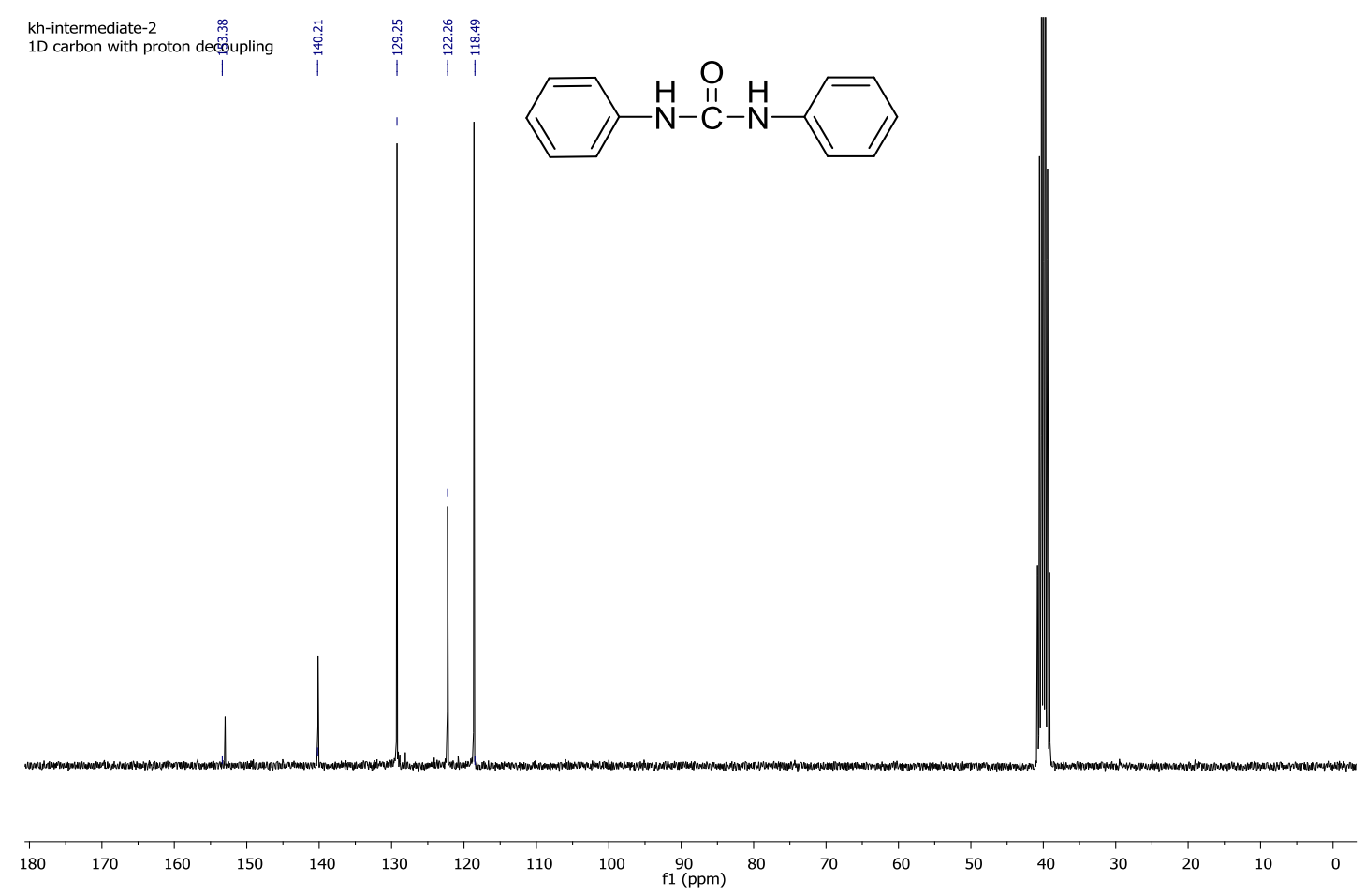

Figure S2.26. The ${ }^{13} \mathrm{C}$ NMR spectrum (75 MHz, DMSO- $\mathrm{d}_{6}$ ) of 1,3-diphenylurea (compound 3) 


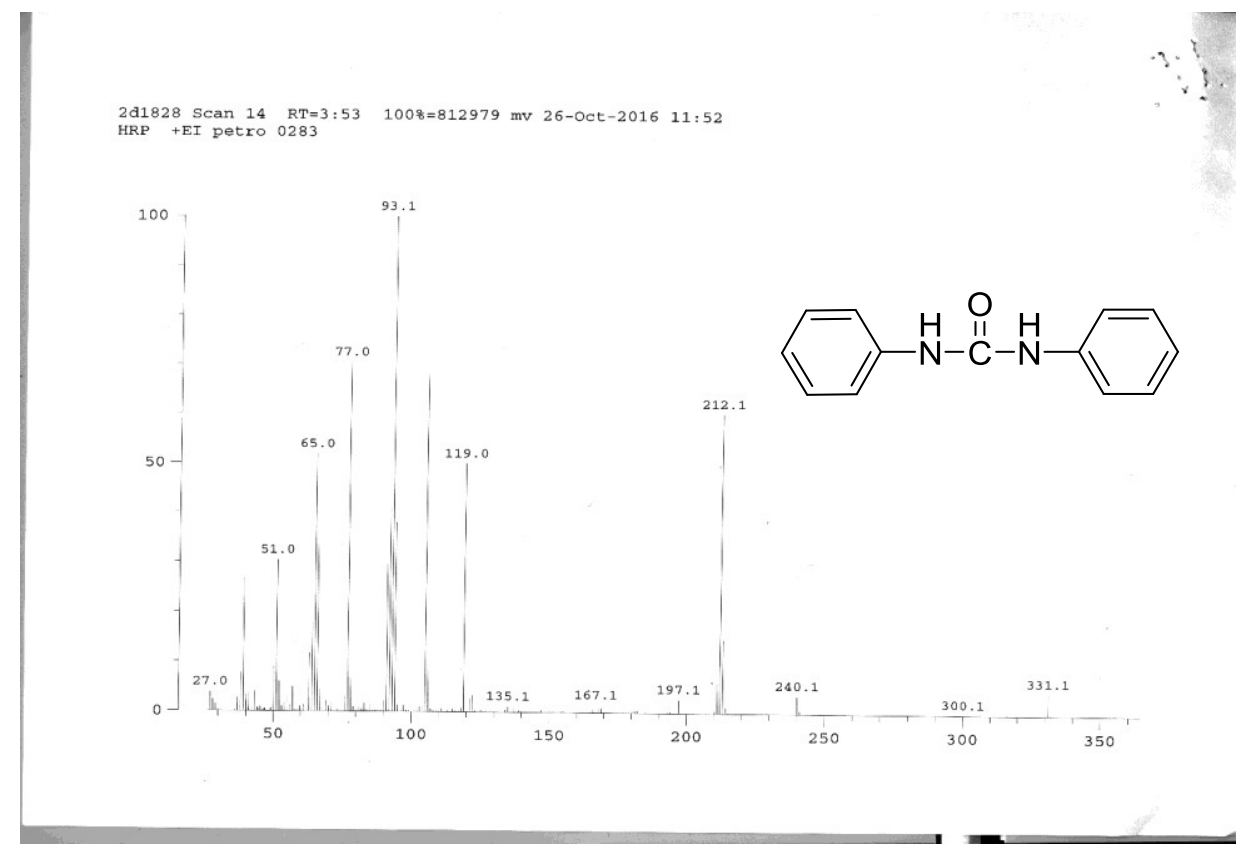

Figure S2.27. EI mass spectrum of 1,3-diphenylurea (compound 3)

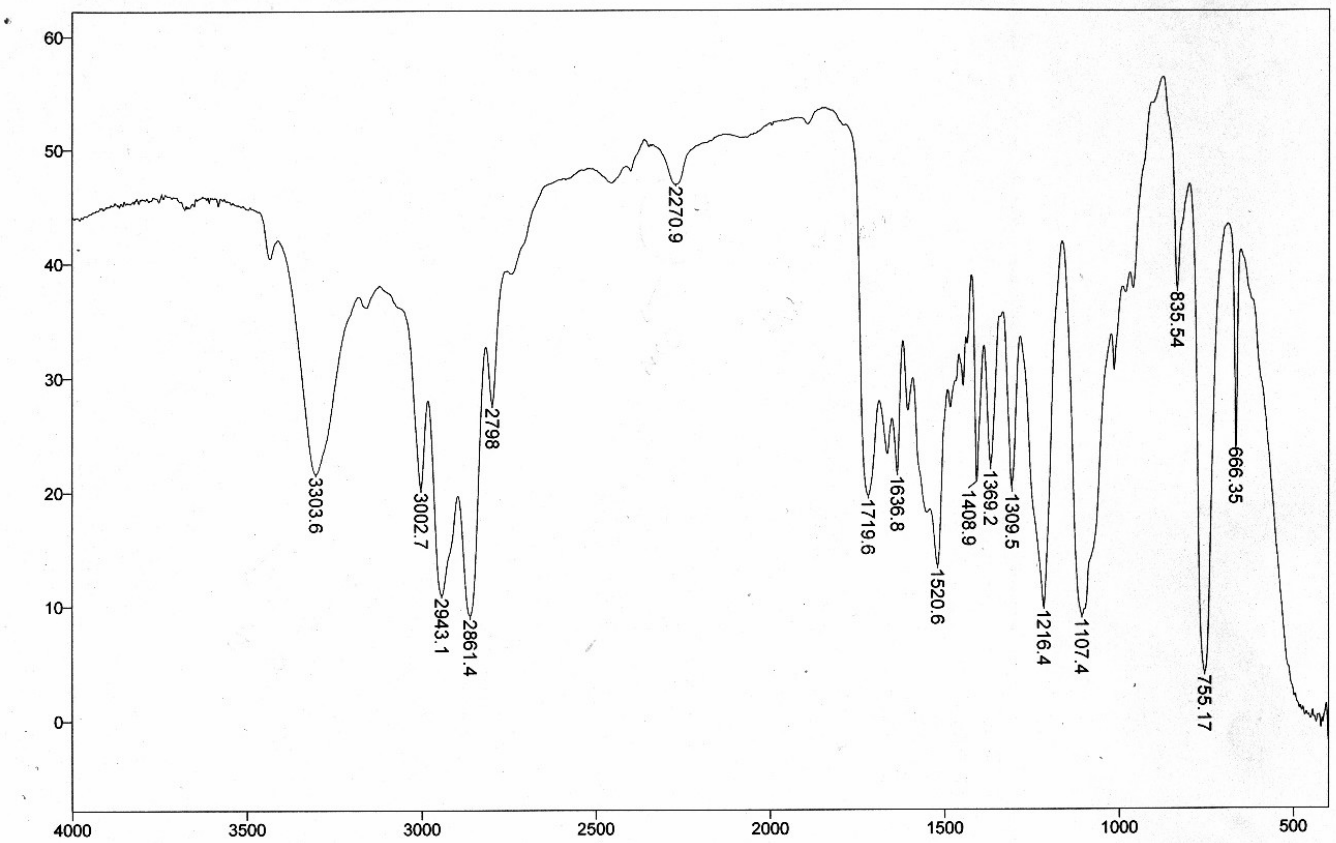

Figure S2.28. IR spectrum of the reaction mixture of DATA and PTMEG after being heated more than 90 minutes 
Appendix B Spectra of Monomers, and Polymers in Chapter 3

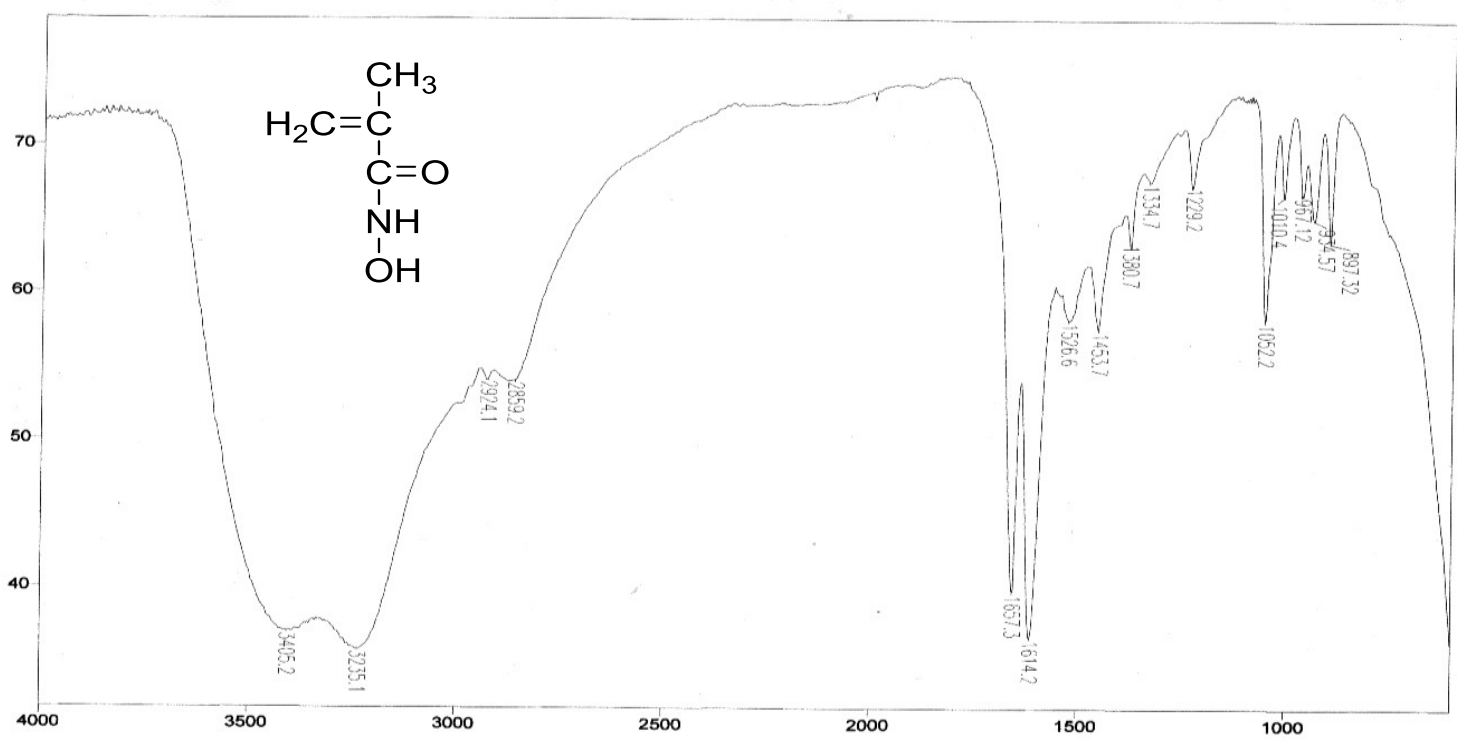

Figure S3.1. IR spectrum of $\mathrm{N}$-hydroxymethacrylamide ( $\mathrm{NaCl}$ plate)

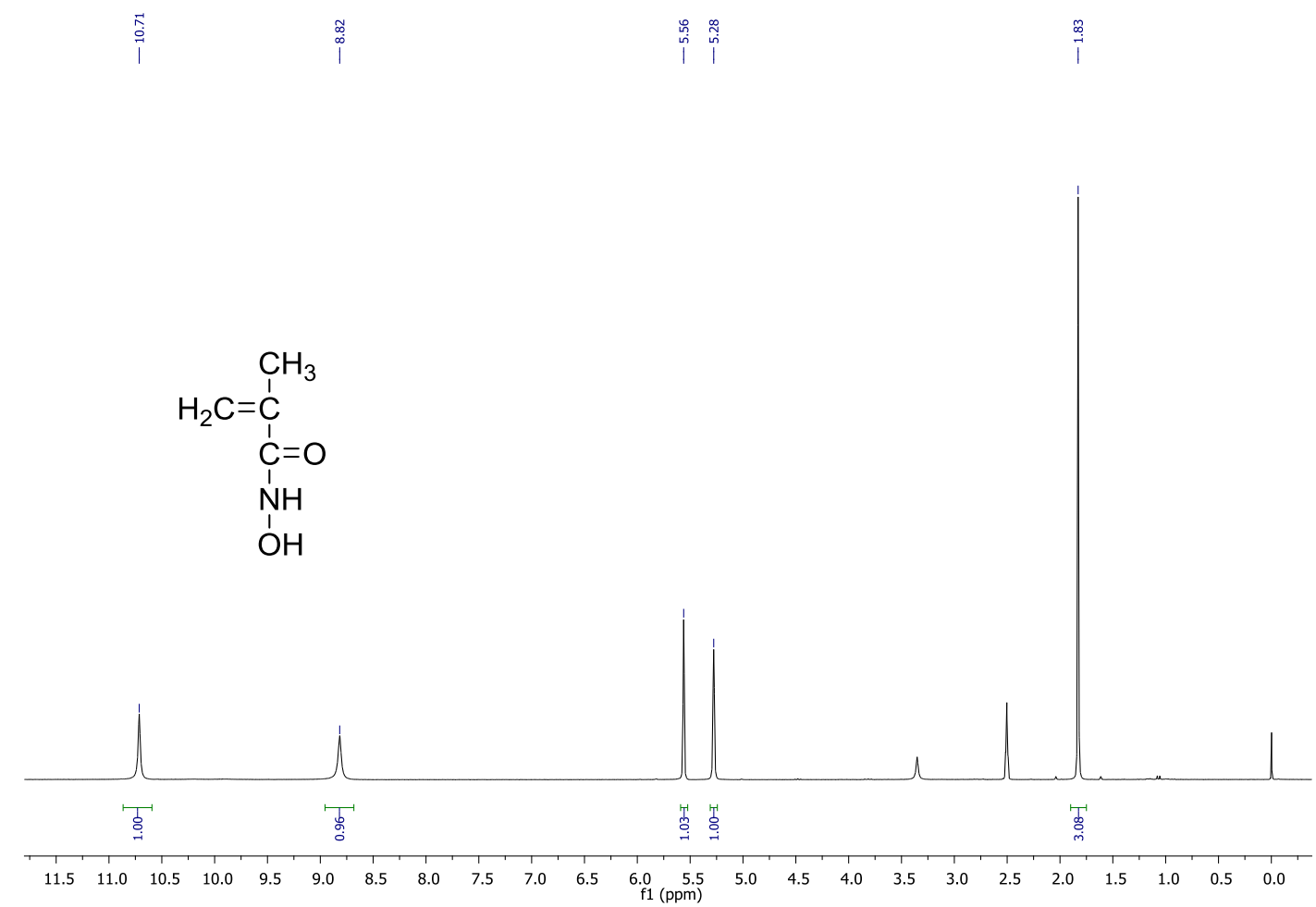


Figure S3.2. The ${ }^{1} \mathrm{H}$ NMR spectrum (300 MHz, DMSO-d ${ }_{6}$ ) of $N$-hydroxymethacrylamide

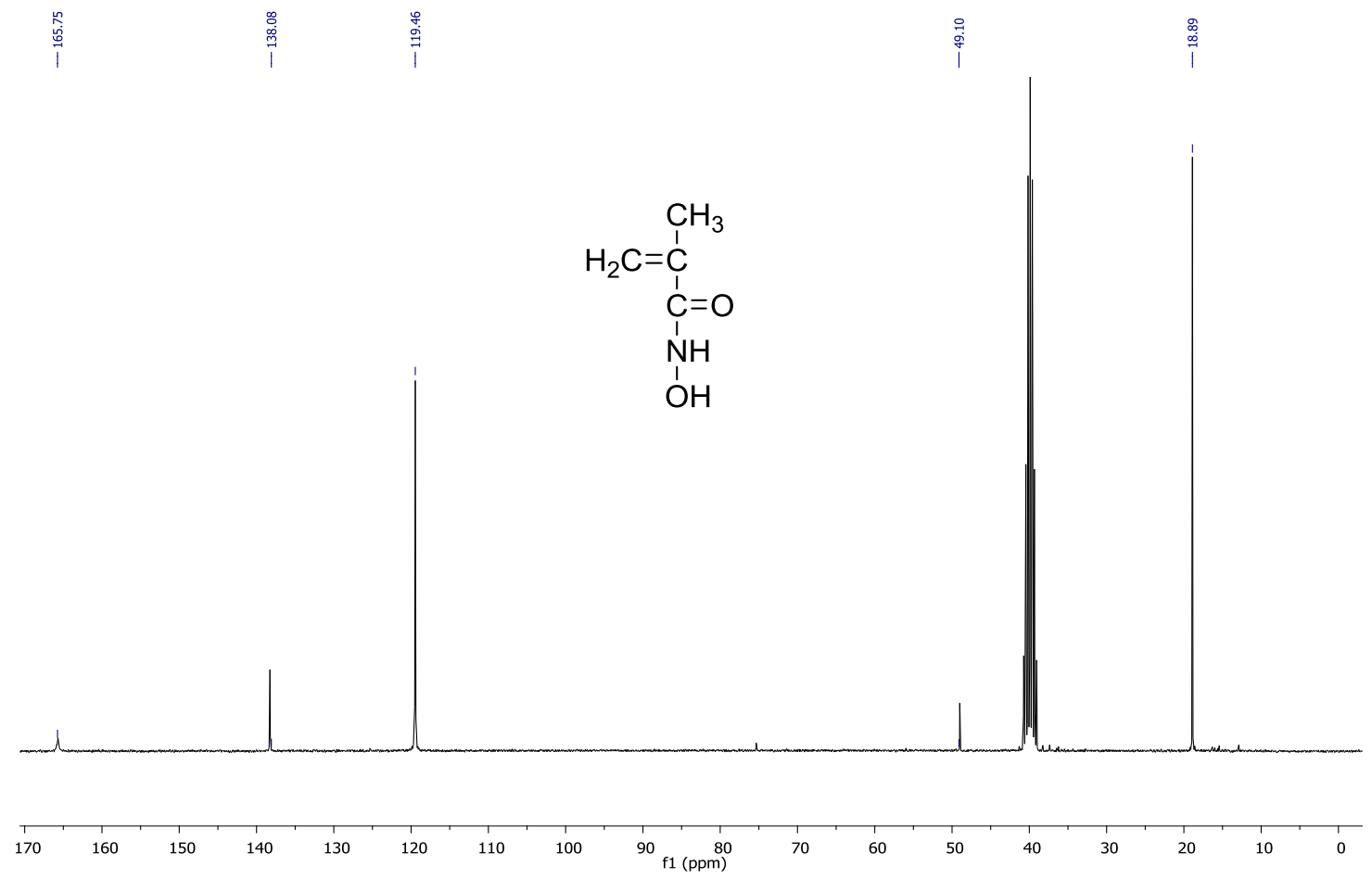

Figure S3.3. The ${ }^{13} \mathrm{C}$ NMR spectrum (75 MHz, DMSO-d $\mathrm{d}_{6}$ ) of $N$-hydroxymethacrylamide

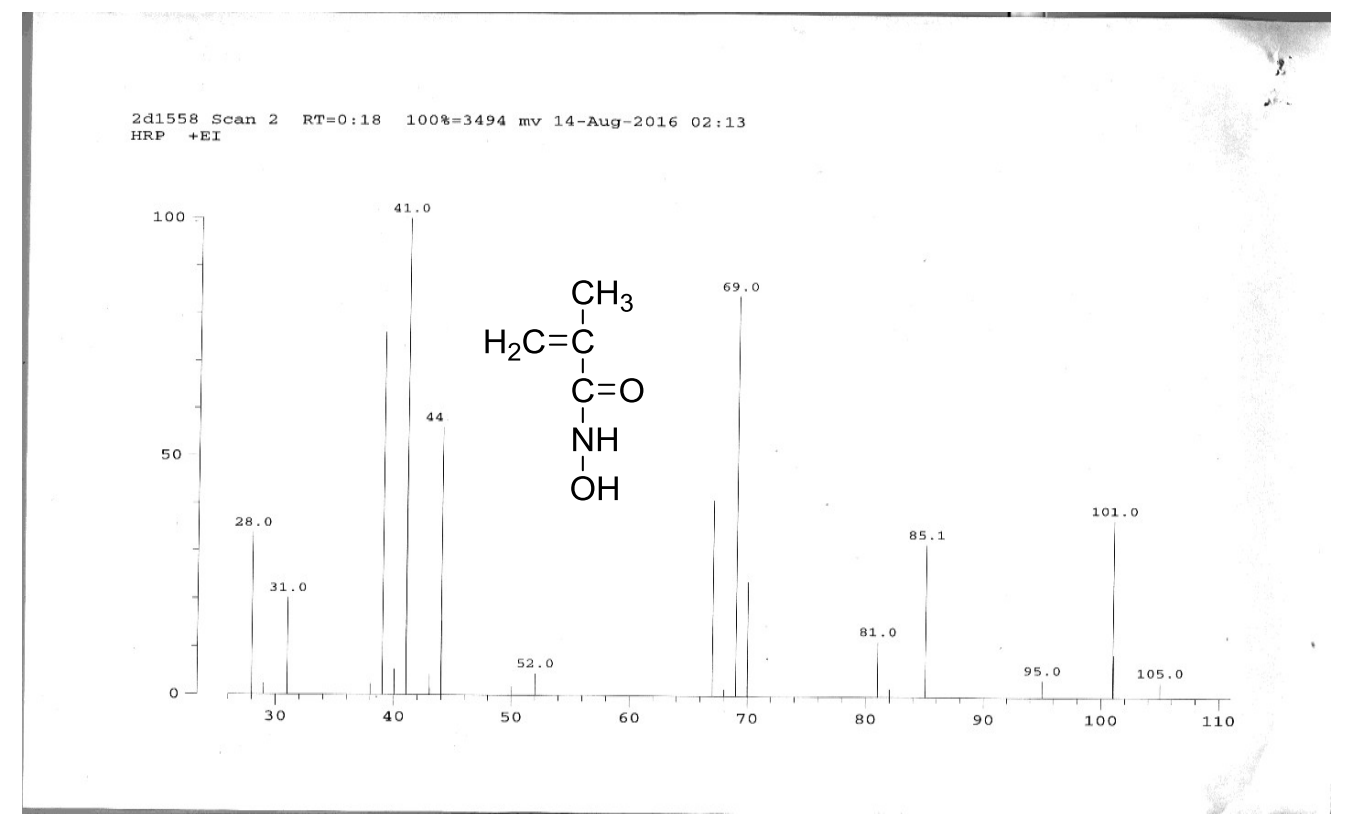

Figure S3.4. EI mass spectrum of $N$-hydroxymethacrylamide 


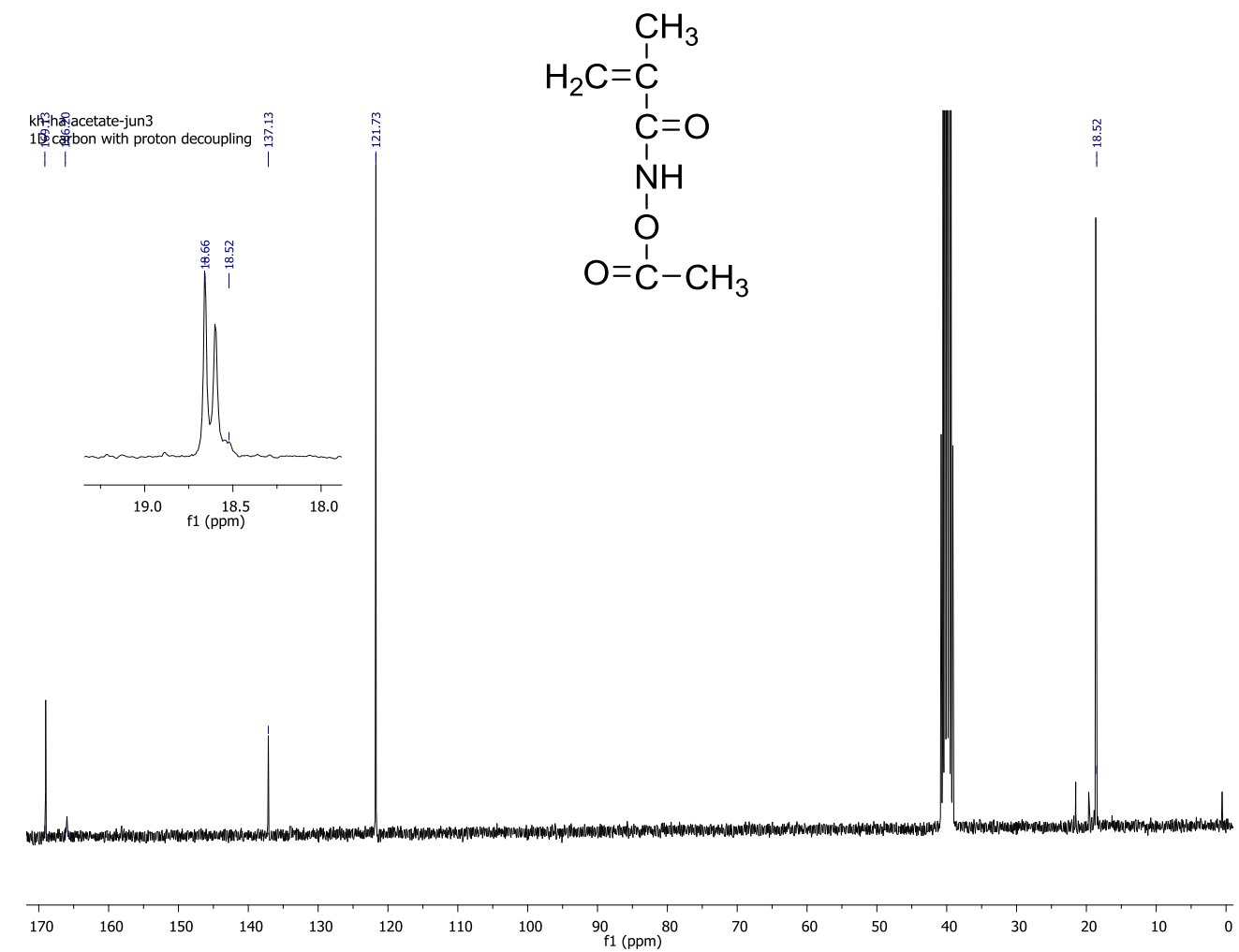

Figure S3.5. The ${ }^{13} \mathrm{C}$ NMR (75 MHz, DMSO- $\mathrm{d}_{6}$ ) of $N$-acetoxymethacrylamide

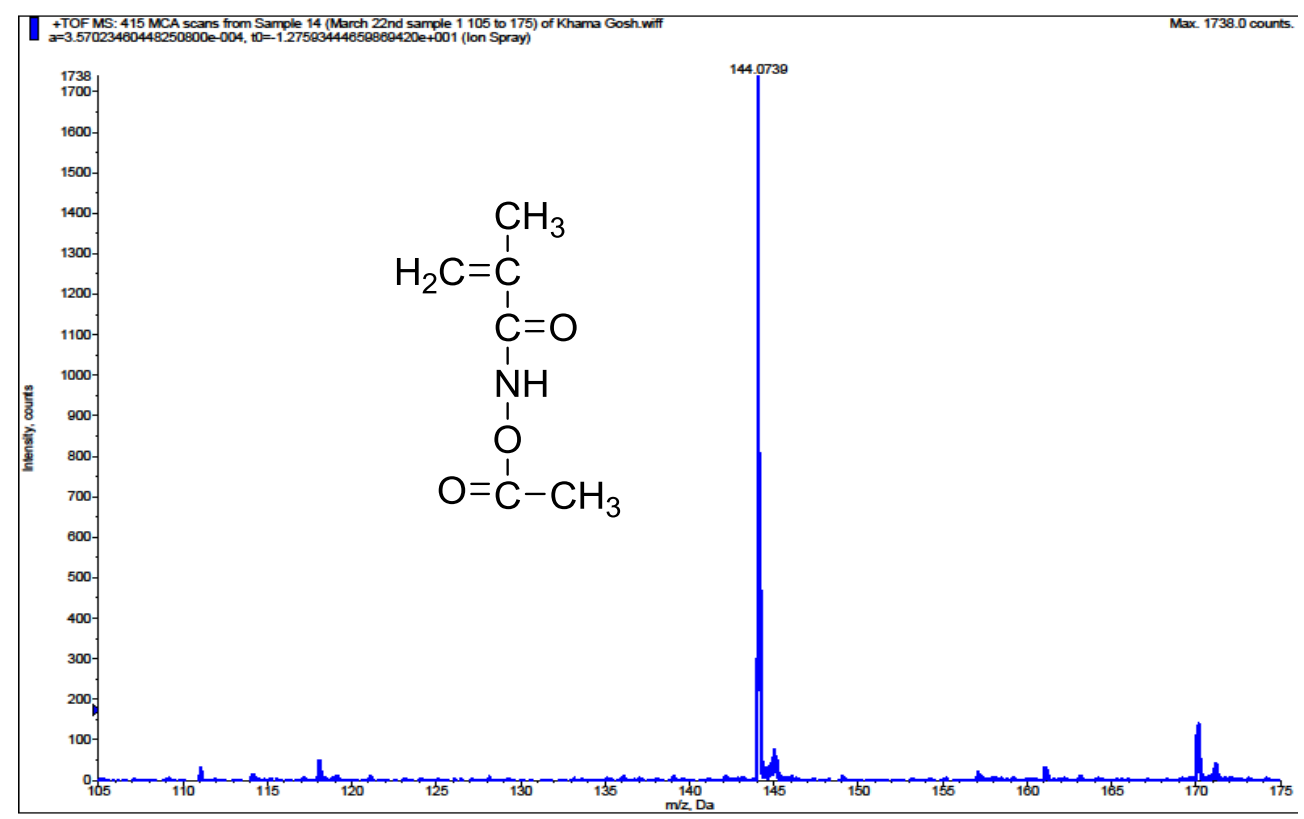

Figure S3.6. ESI mass spectrum of $N$-acetoxymethacrylamide 


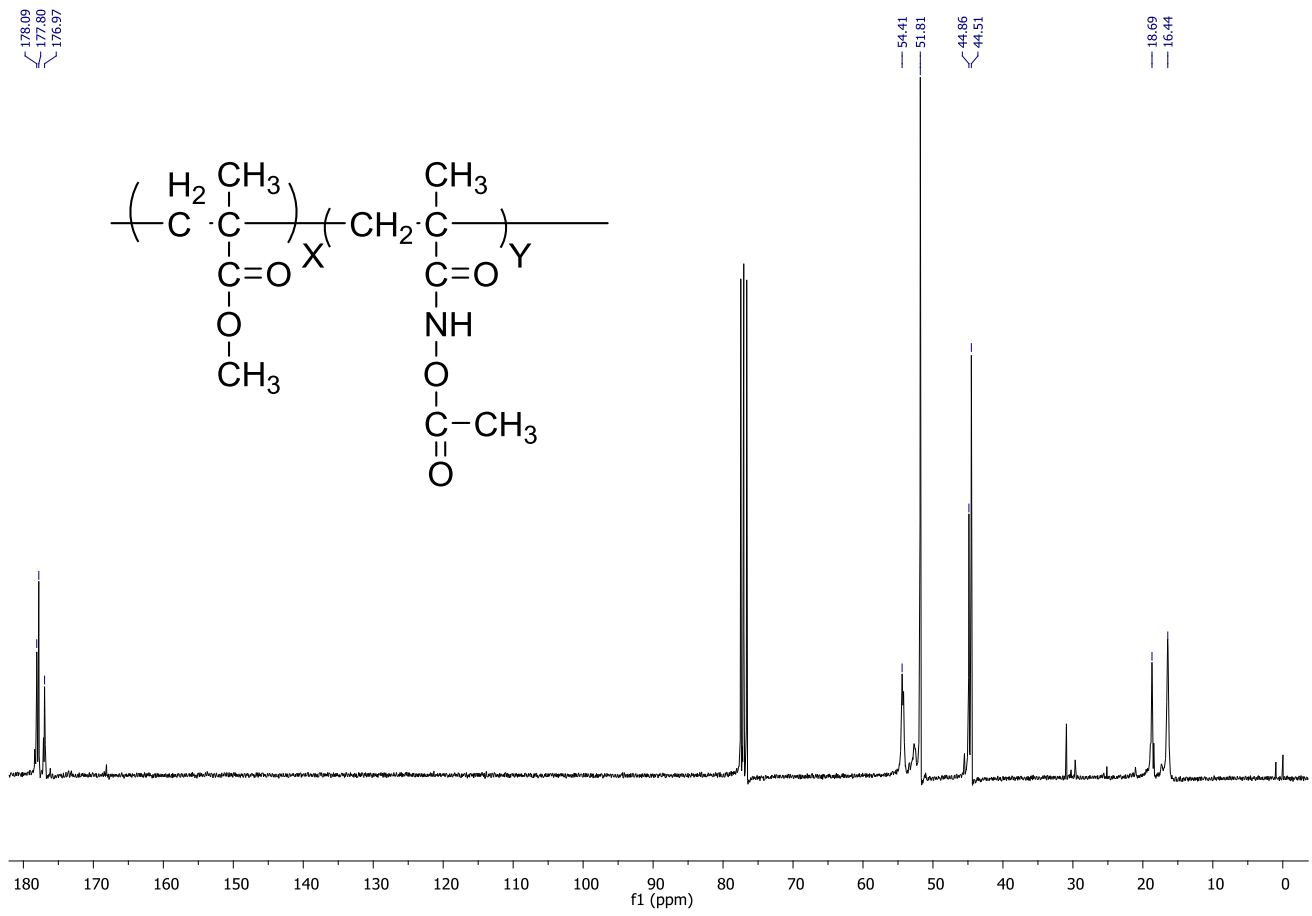

Figure S3.7. The ${ }^{13} \mathrm{C}$ NMR $\left(75 \mathrm{MHz}, \mathrm{CDCl}_{3}\right)$ of $5 \mathrm{~mol} \%$ of $\mathrm{N}$-acetoxymethacrylamide containing polymethacrylate (P5)

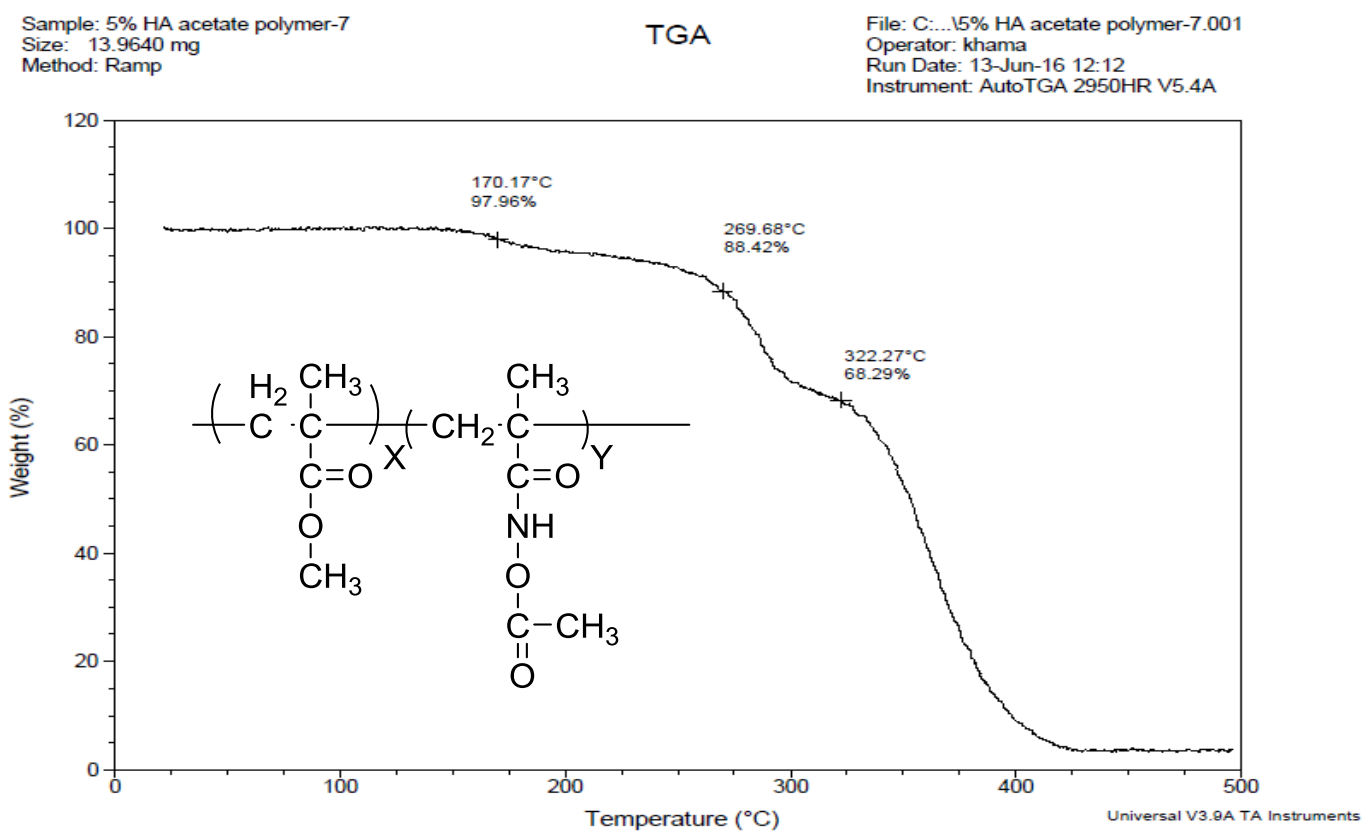

Figure S3.8. TGA trace of $5 \mathrm{~mol} \% \mathrm{~N}$-acetoxymethacrylamide -containing polymethacrylate (P5) 


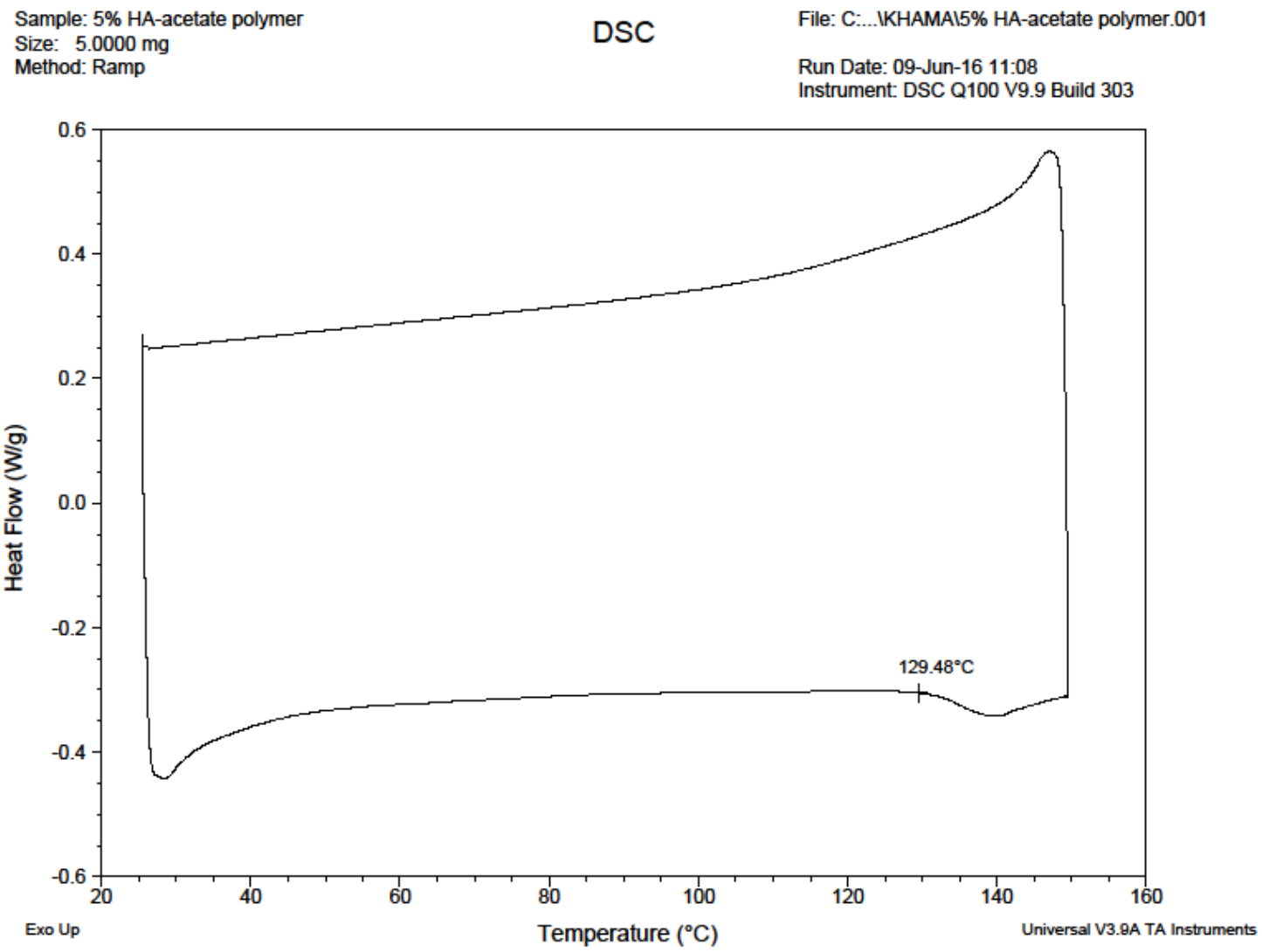

Figure S3.9. DSC trace of $5 \mathrm{~mol} \% \mathrm{~N}$-acetoxymethacrylamide -containing polymethacrylate (P5)

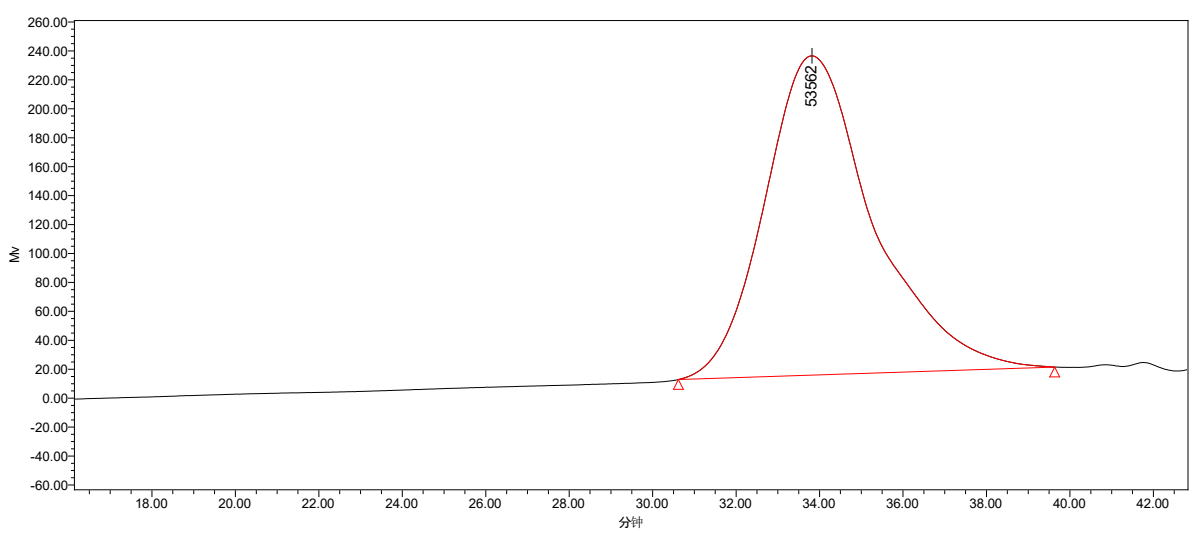

$\begin{array}{llllll}\text { Mn } & \text { Mw } & \text { MP } & \text { Mv } & \text { Mz } & \mathrm{Mz}+1\end{array}$

$24151.28 \quad 64235.38 \quad 53562.17 \quad 56846.16 \quad 127063 \quad 206328.2$

Figure S3.10. GPC of $5 \mathrm{~mol} \% \mathrm{~N}$-acetoxymethacrylamide -containing polymethacrylate (P5) 

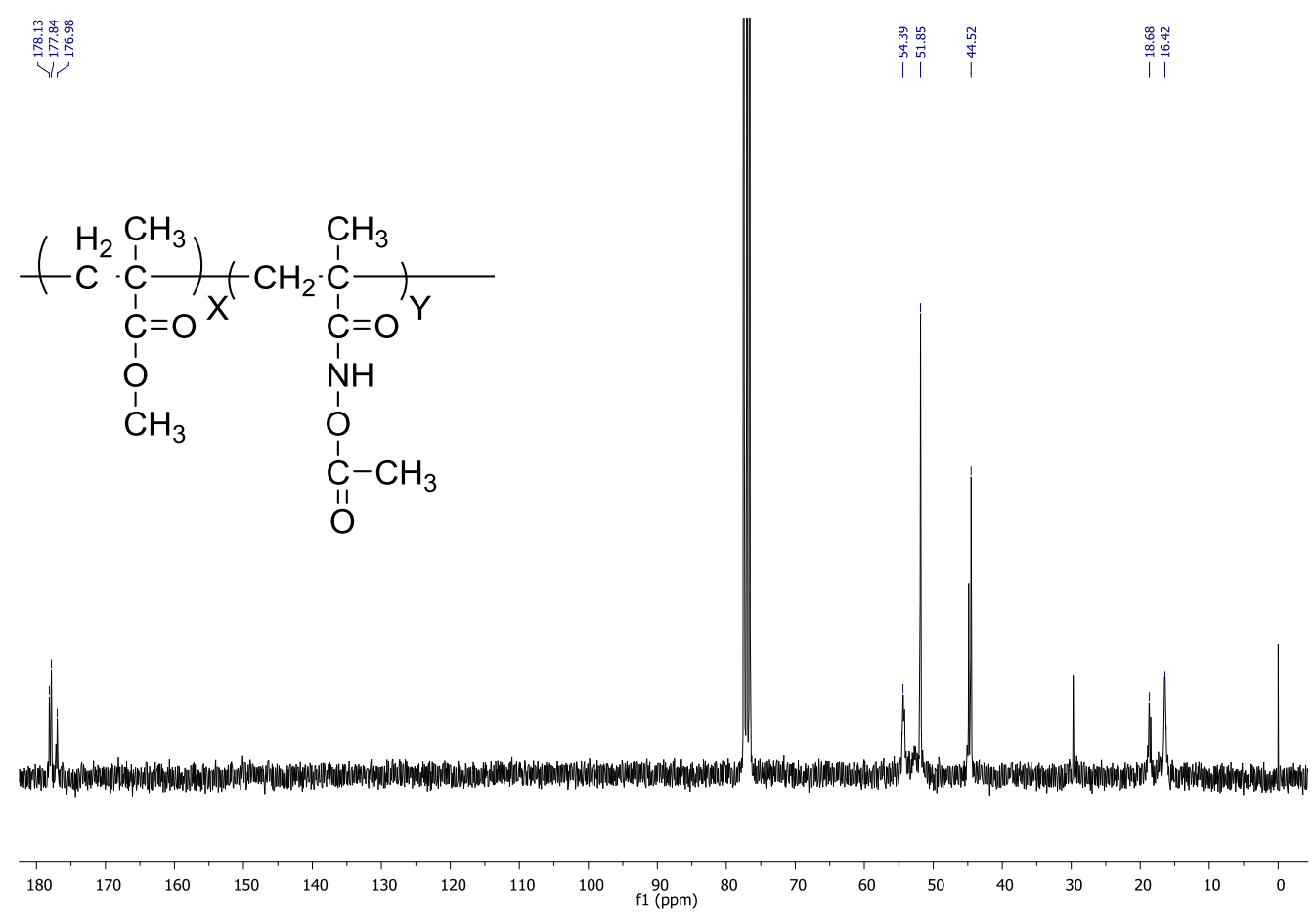

Figure S3.11. The ${ }^{13} \mathrm{C} \mathrm{NMR}\left(75 \mathrm{MHz}, \mathrm{CDCl}_{3}\right)$ of $10 \mathrm{~mol} \% \mathrm{~N}$-acetoxymethacrylamide containing polymethacrylate (P6)

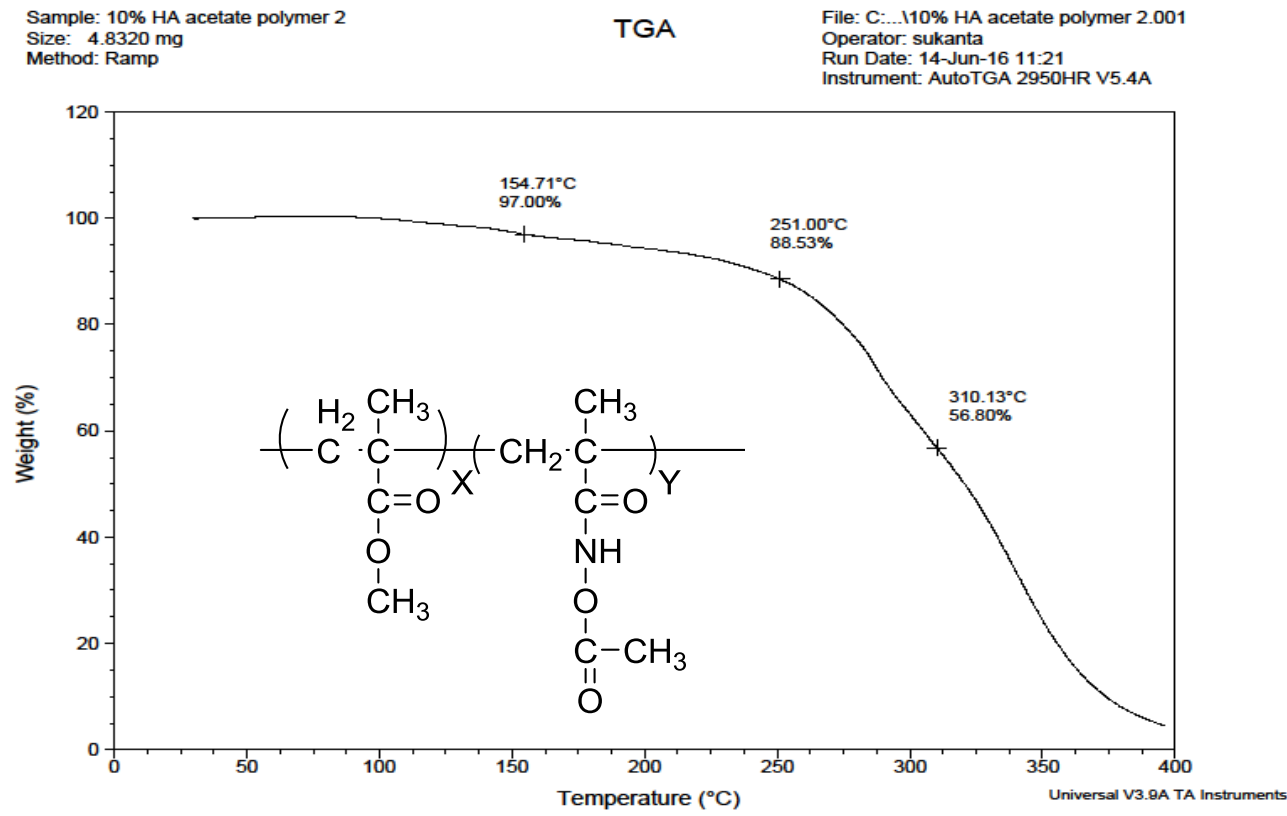

Figure S3.12. TGA trace of $10 \mathrm{~mol} \% \mathrm{~N}$-acetoxymethacrylamide -containing polymethacrylate (P6) 


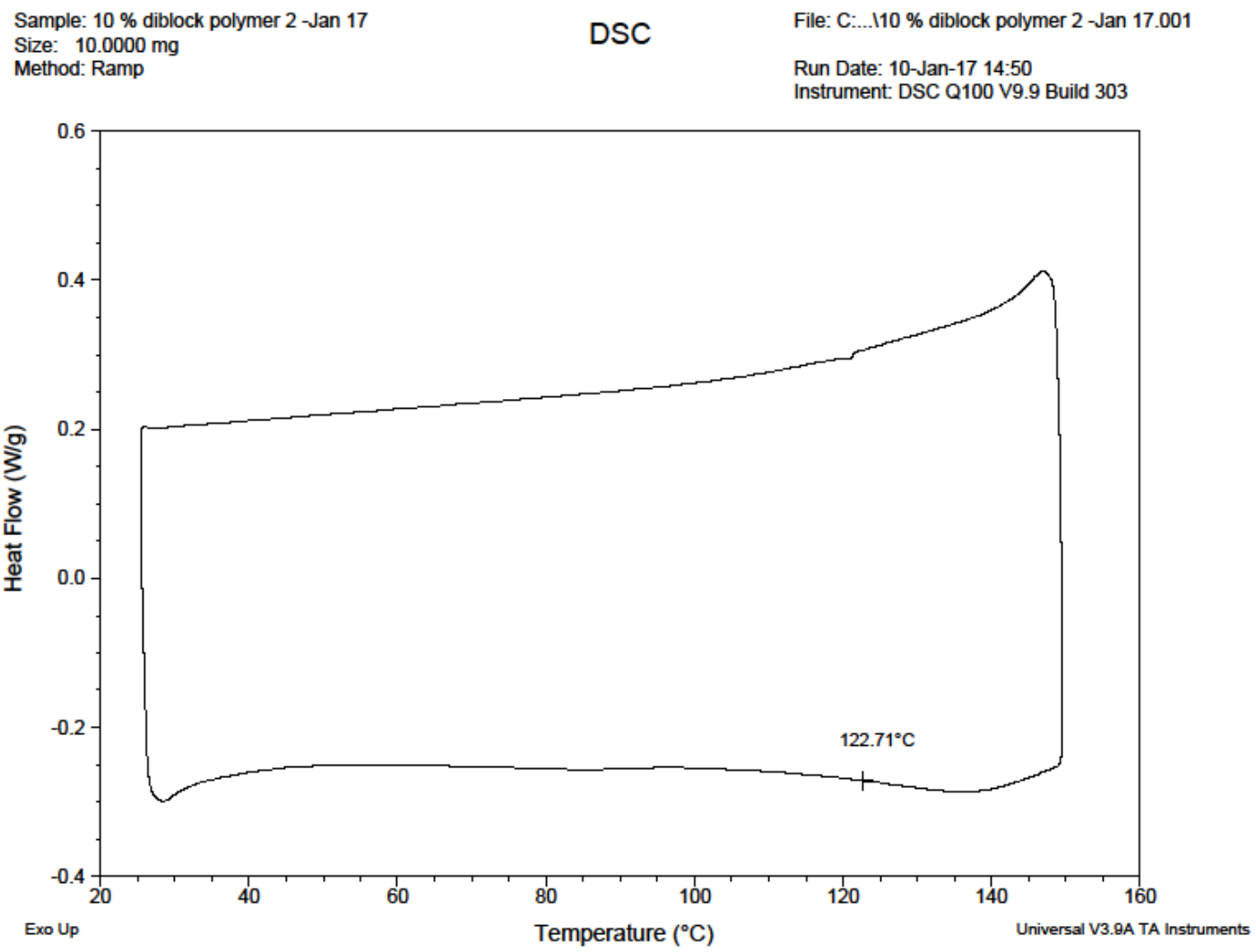

Figure S3.13. DSC trace of $10 \mathrm{~mol} \% \mathrm{~N}$-acetoxymethacrylamide -containing polymethacrylate (P6)

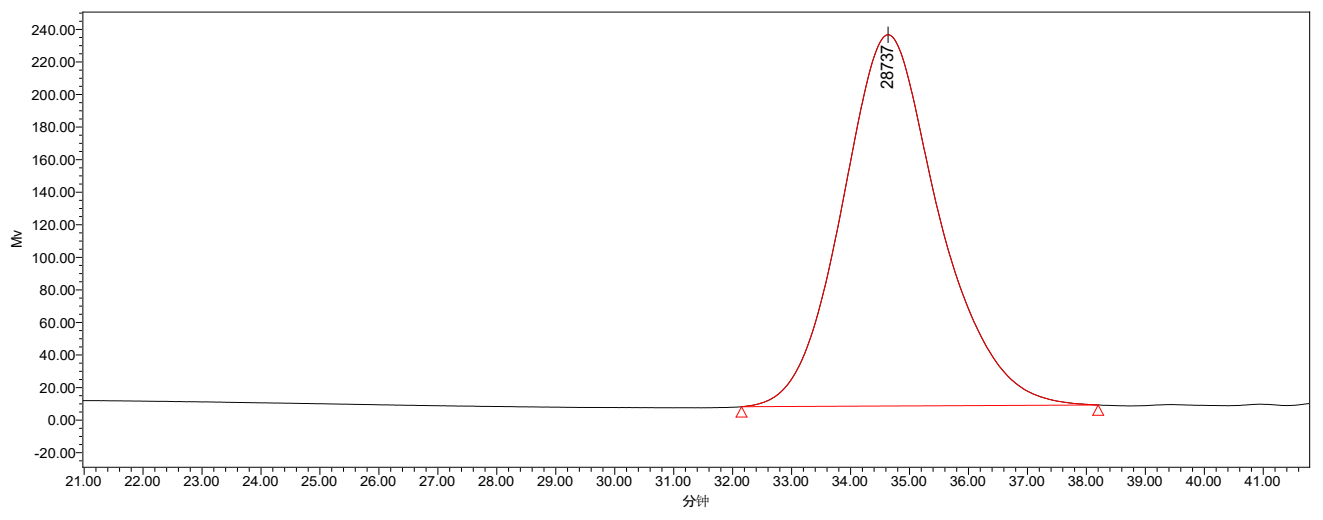

$\begin{array}{llllll}\text { Mn } & \text { Mw } & \text { MP } & \text { Mv } & \text { Mz } & \mathrm{Mz}+1\end{array}$

$22291.75 \quad 31811.4 \quad 28736.99 \quad 30203.82 \quad 43883.56 \quad 58786.78$

Figure S3.14. GPC of $10 \mathrm{~mol} \% \mathrm{~N}$-acetoxymethacrylamide -containing polymethacrylate (P6) 


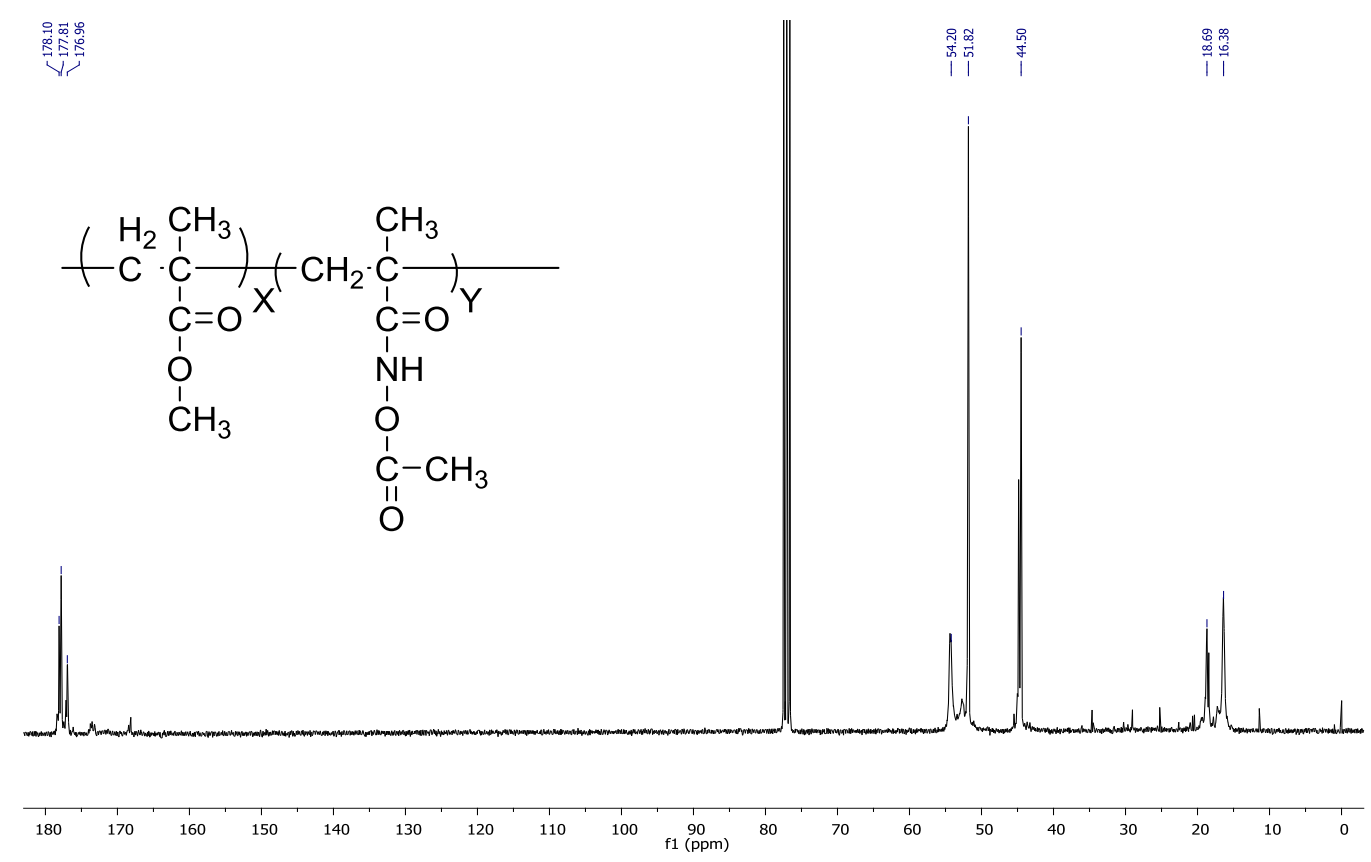

Figure S3.15. The ${ }^{13} \mathrm{C}$ NMR $\left(75 \mathrm{MHz}, \mathrm{CDCl}_{3}\right)$ of $20 \mathrm{~mol} \% \mathrm{~N}$-acetoxymethacrylamide containing polymethacrylate (P7)

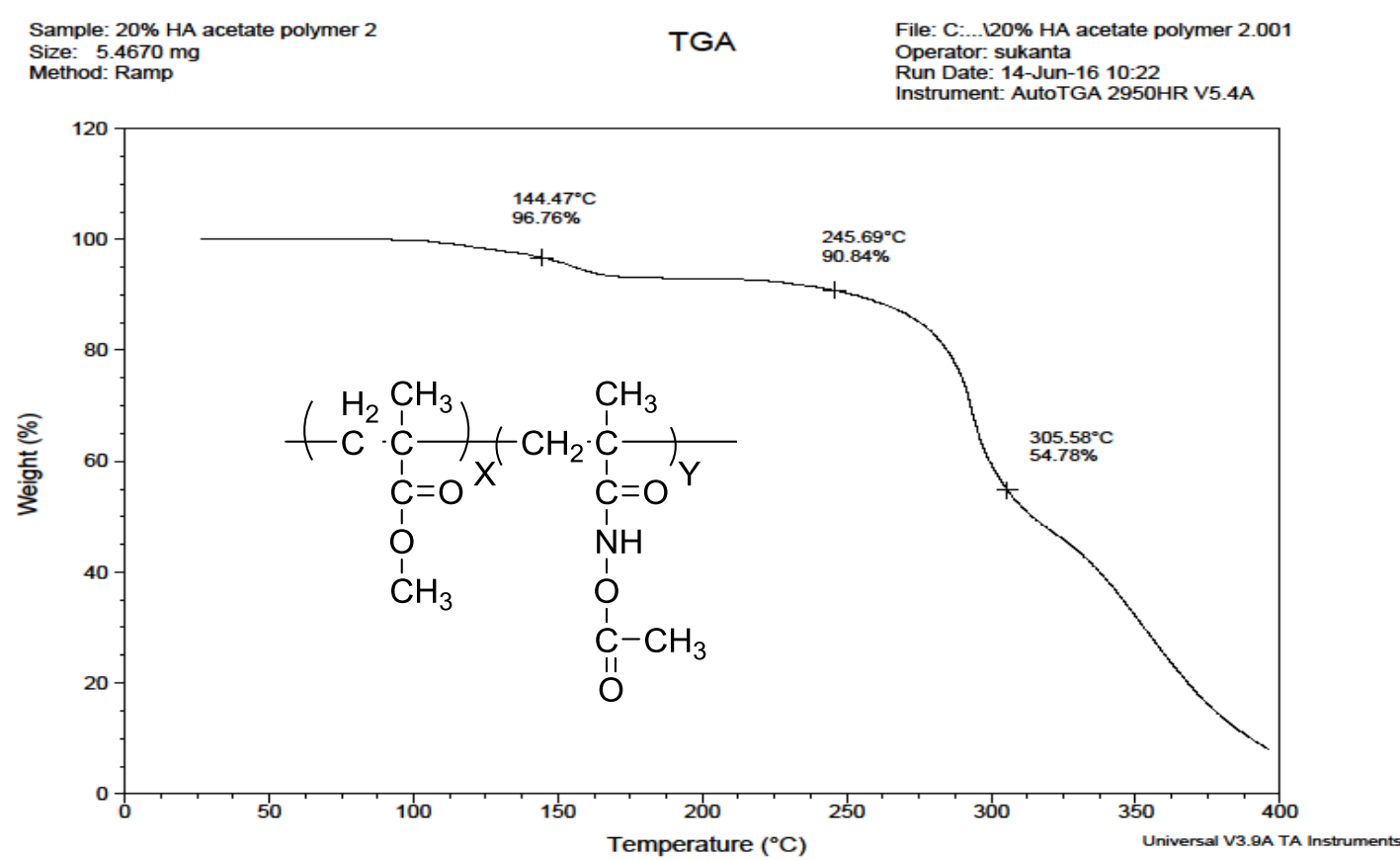

Figure S3.16. TGA trace of $20 \mathrm{~mol} \% \mathrm{~N}$-acetoxymethacrylamide -containing polymethacrylate (P7) 


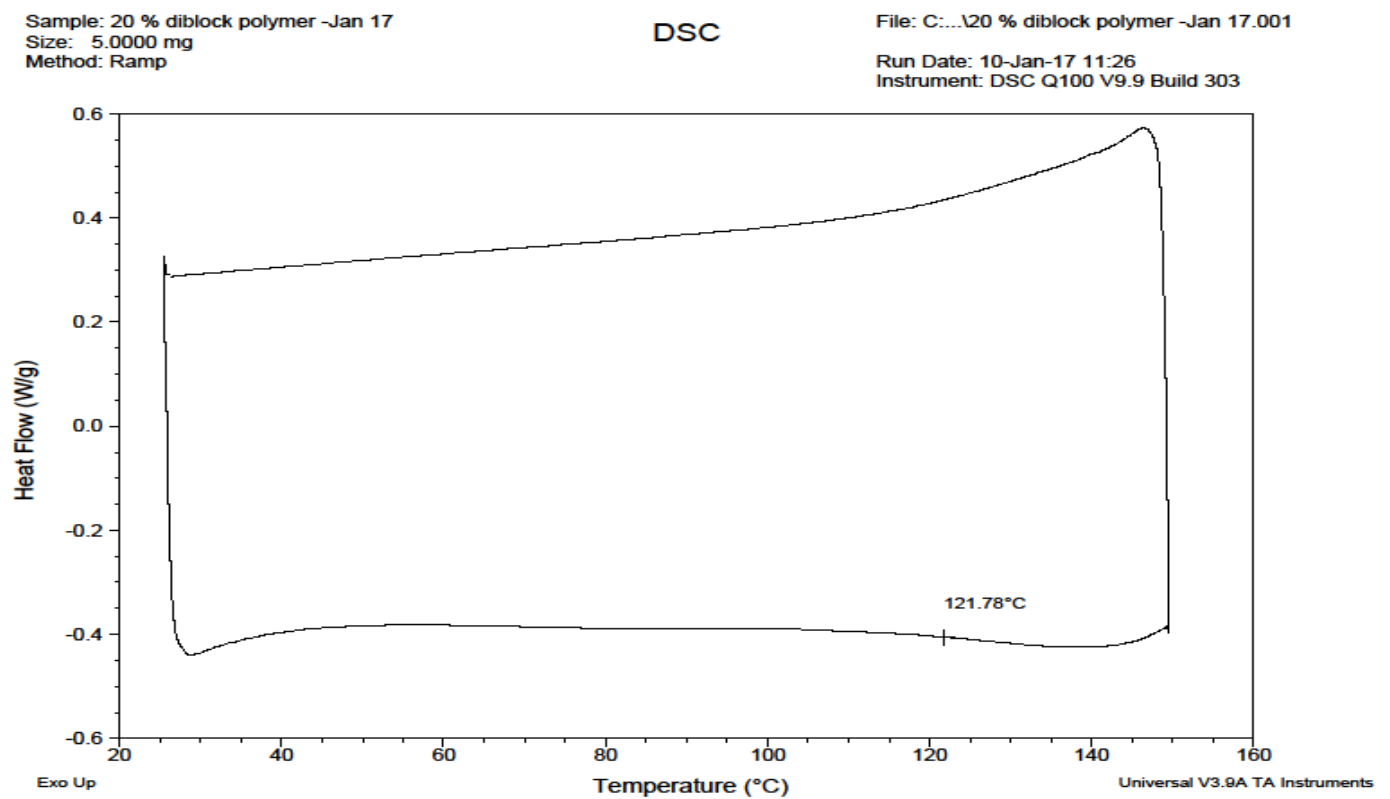

Figure S3.17. DSC trace of $20 \mathrm{~mol} \% \mathrm{~N}$-acetoxymethacrylamide -containing polymethacrylate (P7)

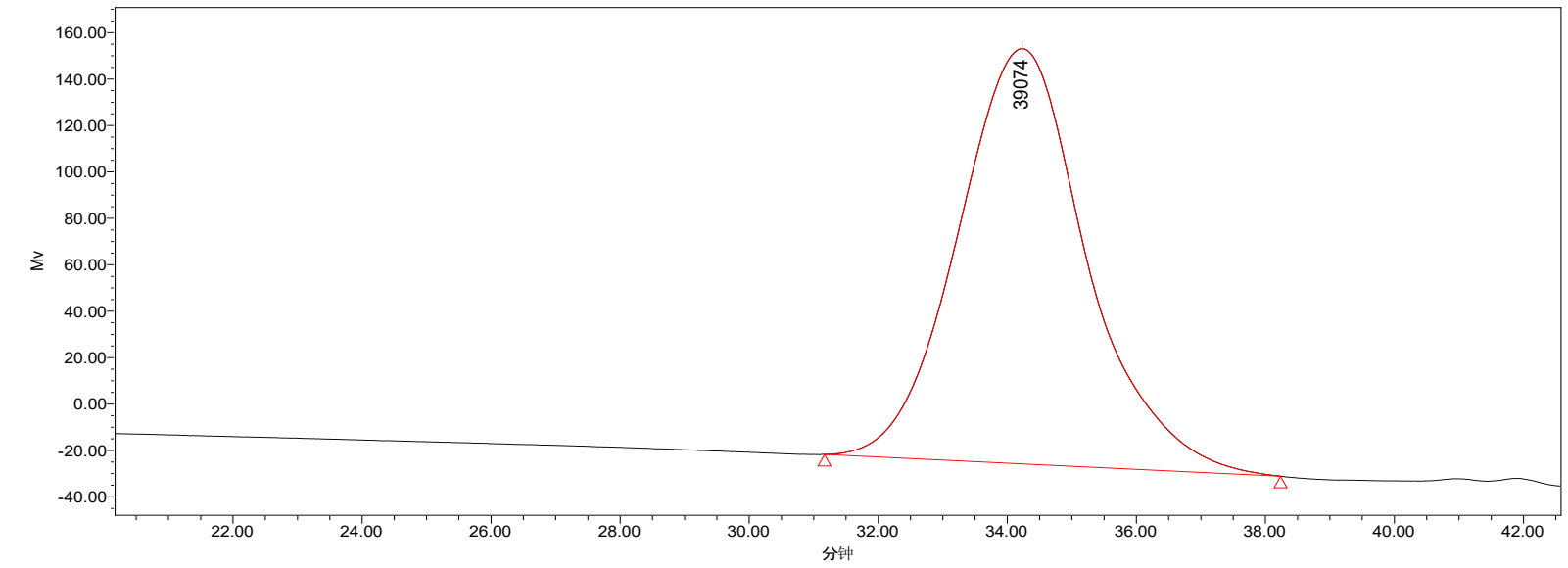

$\begin{array}{llllll}\text { Mn } & \mathrm{Mw} & \mathrm{MP} & \mathrm{Mv} & \mathrm{Mz} & \mathrm{Mz}+1\end{array}$

28618.38 48938.45 $39074.28 \quad 45261.31 \quad 79223.9 \quad 120345$

Figure S3.18. GPC of $20 \mathrm{~mol} \% \mathrm{~N}$-acetoxymethacrylamide -containing polymethacrylate (P7) 


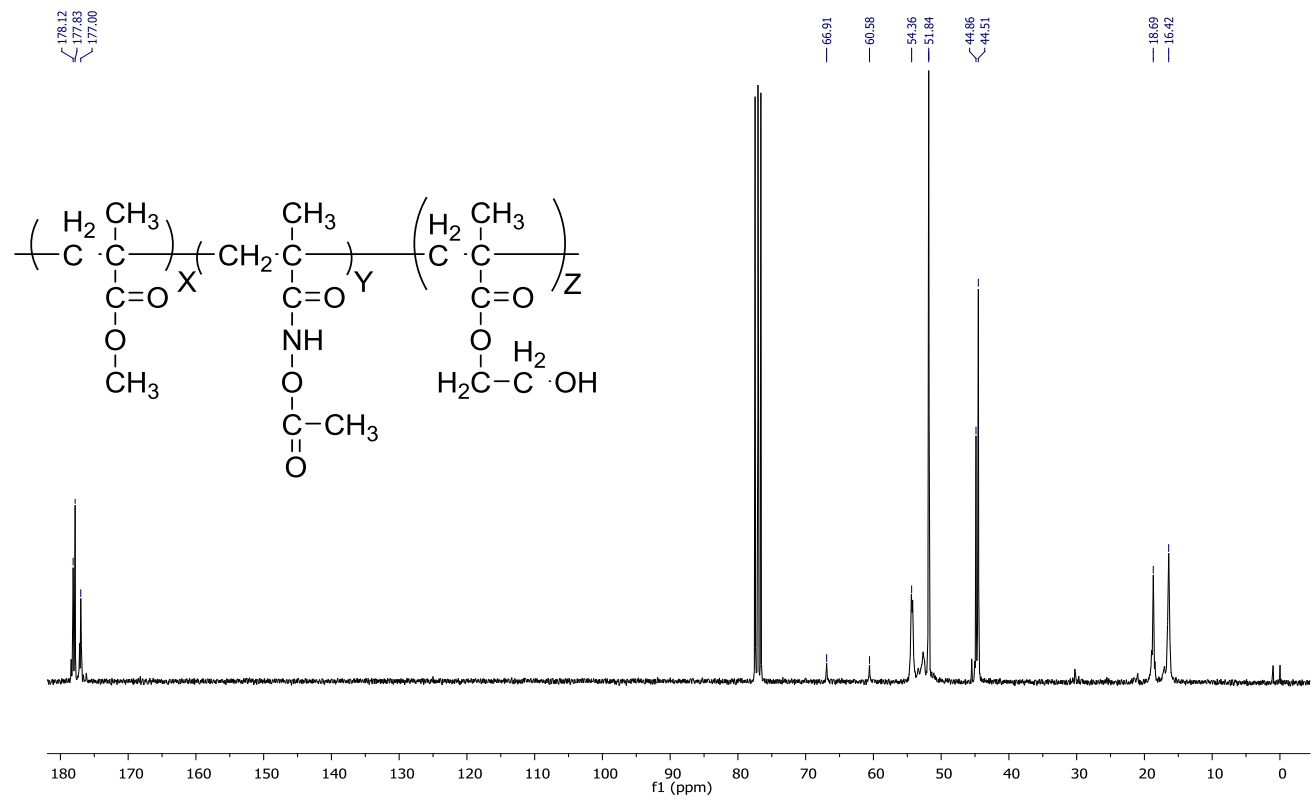

Figure 3.19. The ${ }^{13} \mathrm{C}$ NMR (75 MHz, $\mathrm{CDCl}_{3}$ ) of $5 \mathrm{~mol} \%$ terpolymer (P12)

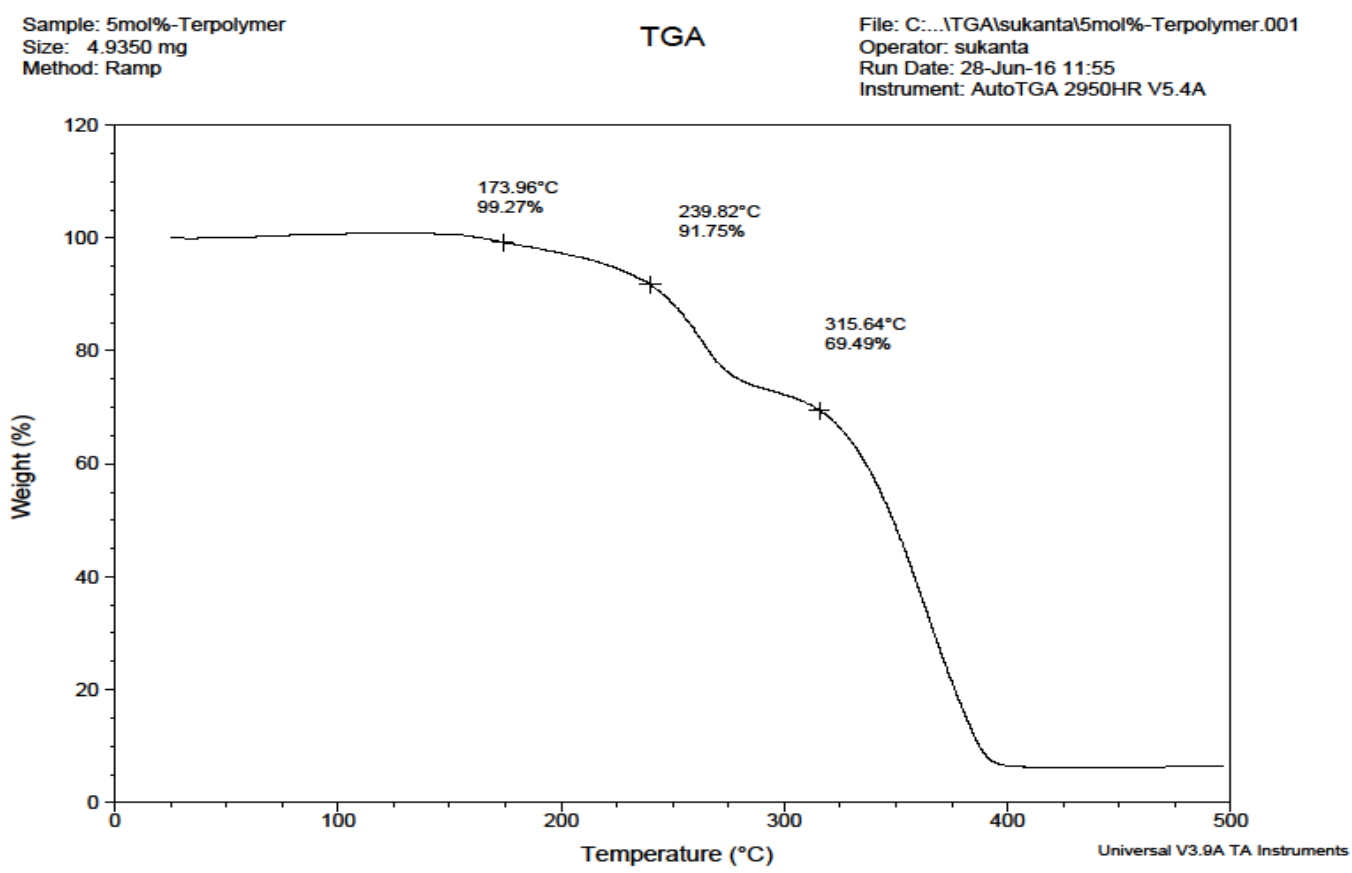

Figure S3.20. TGA trace of $5 \mathrm{~mol} \%$ terpolymer (P12) 


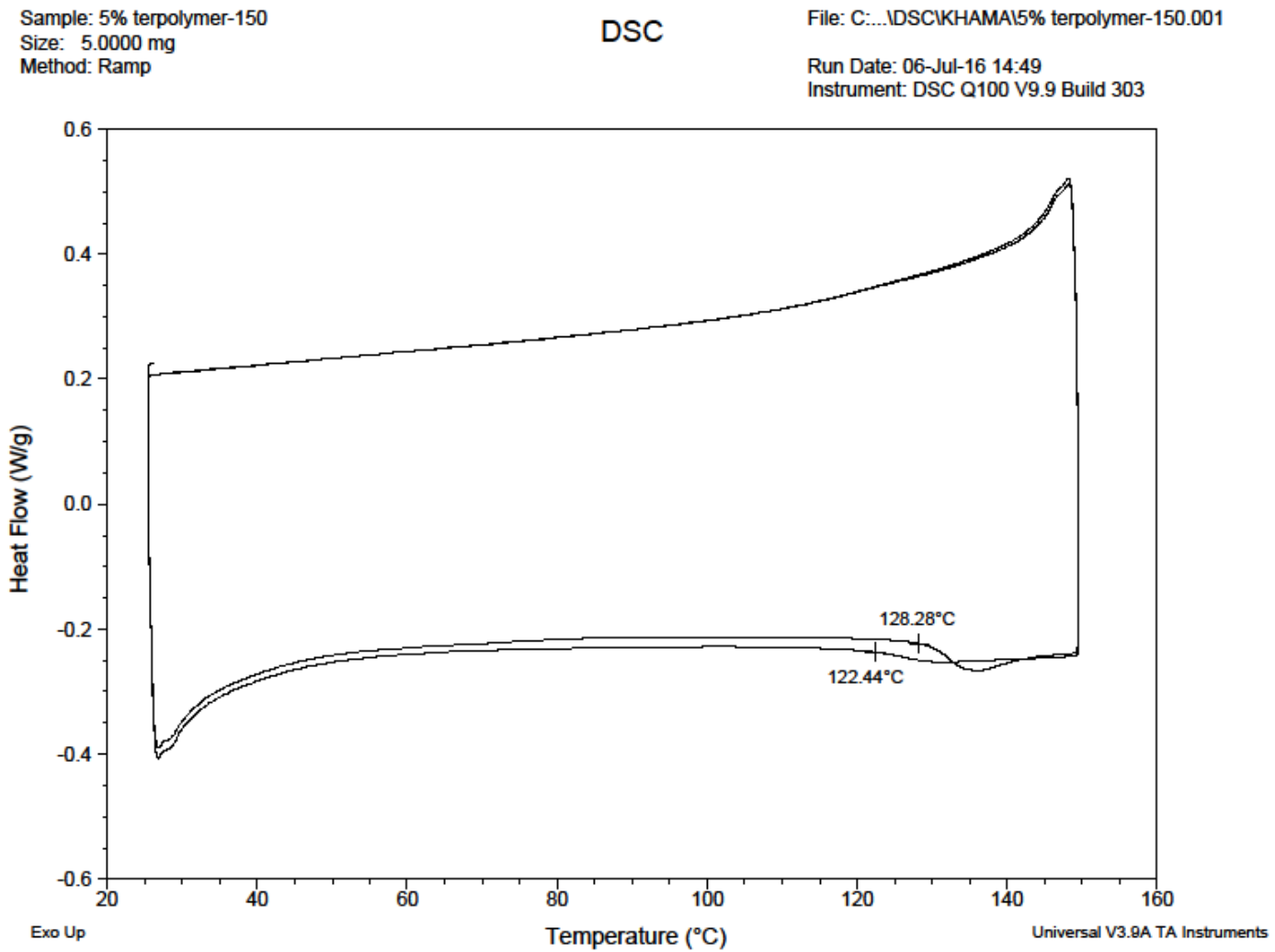

Figure S3.21. DSC trace of $5 \mathrm{~mol} \%$ terpolymer (P12)

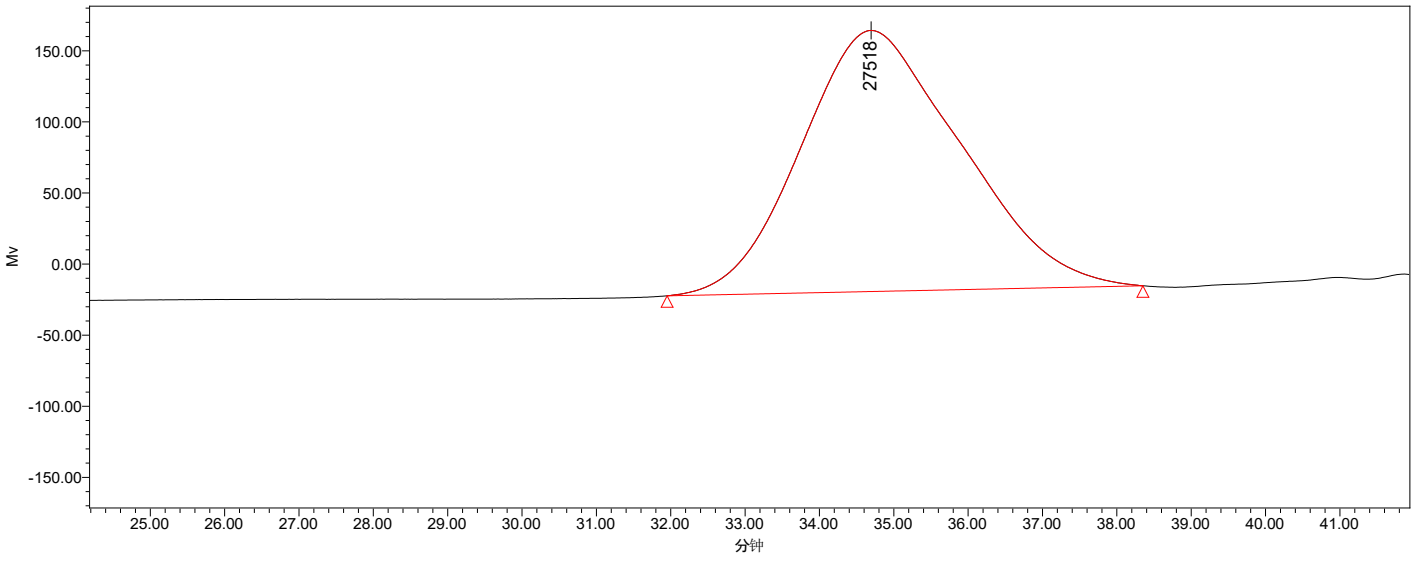

$\begin{array}{lllllll}\text { Mn } & \text { Mw } & \text { MP } & \text { Mv } & \text { Mz } & \mathrm{Mz}+1\end{array}$

$\begin{array}{llllll}18150.65 & 30770.88 & 27518.2 & 28470.76 & 49032.27 & 71530.79\end{array}$

Figure S3.22. GPC of of $5 \mathrm{~mol} \%$ terpolymer (P12) 

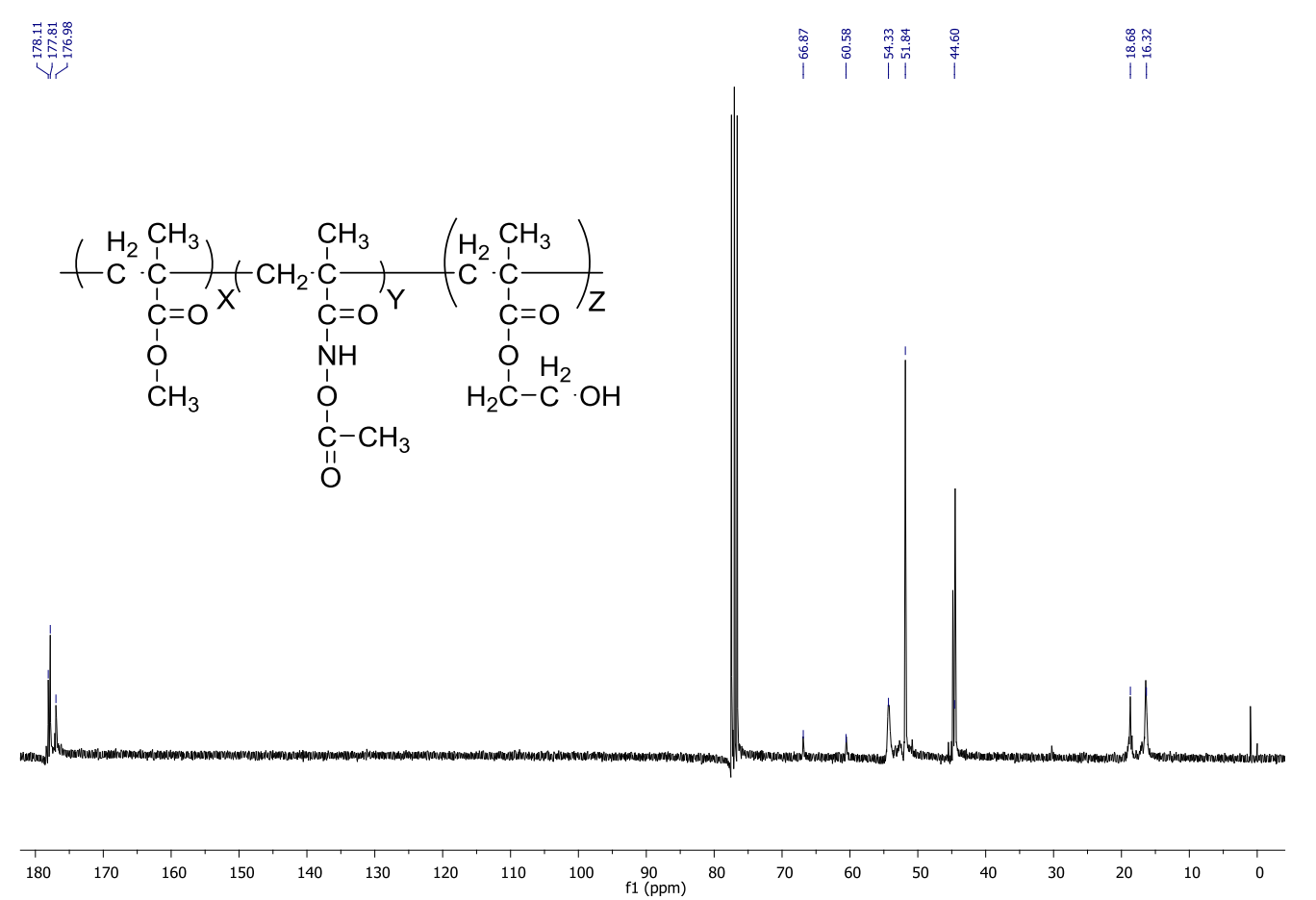

Figure S3.23. The ${ }^{13} \mathrm{C}$ NMR (75 MHz, $\mathrm{CDCl}_{3}$ ) of $10 \mathrm{~mol} \%$ terpolymer (P13)

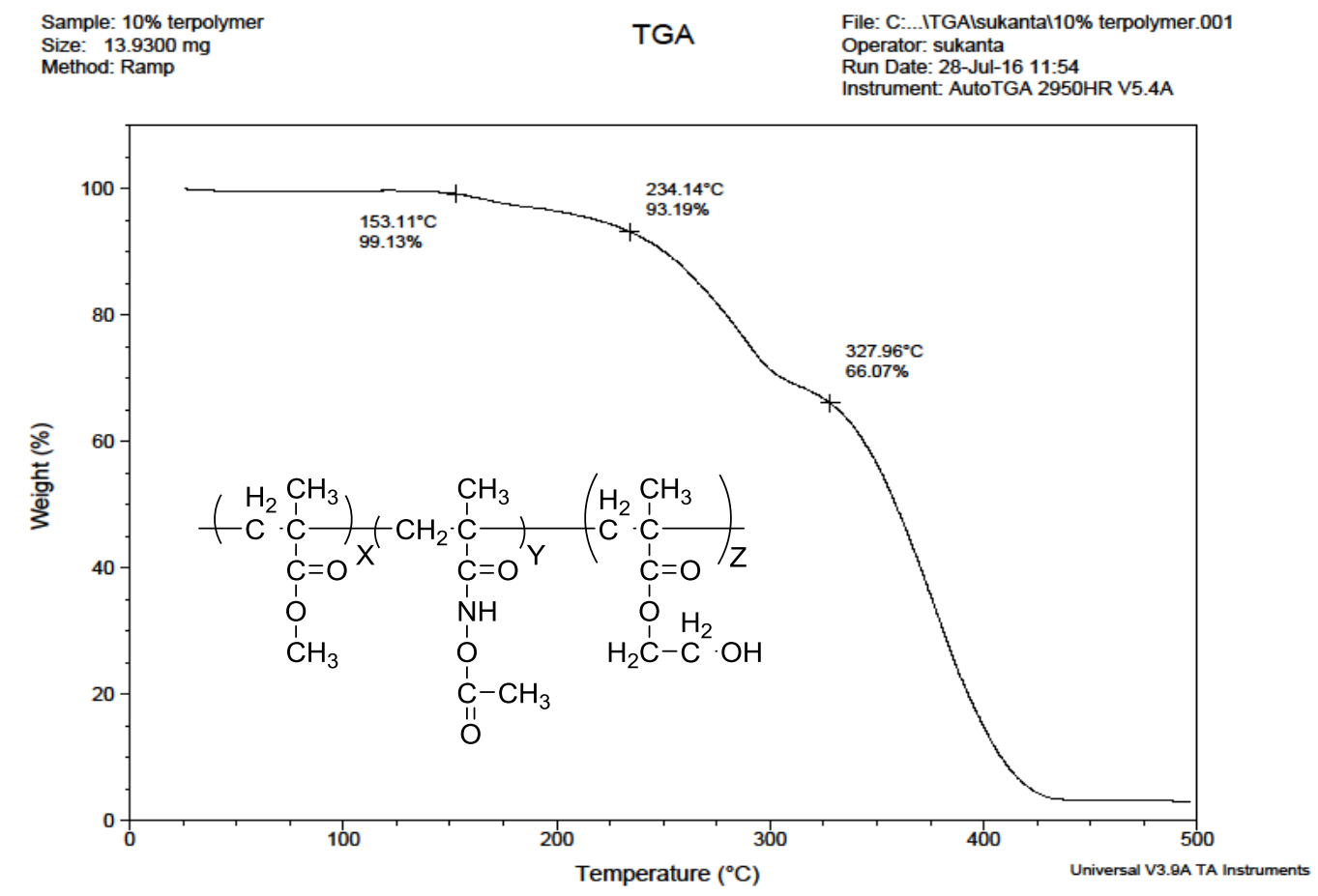

Figure S3.24. TGA trace of $10 \mathrm{~mol} \%$ terpolymer (P13) 


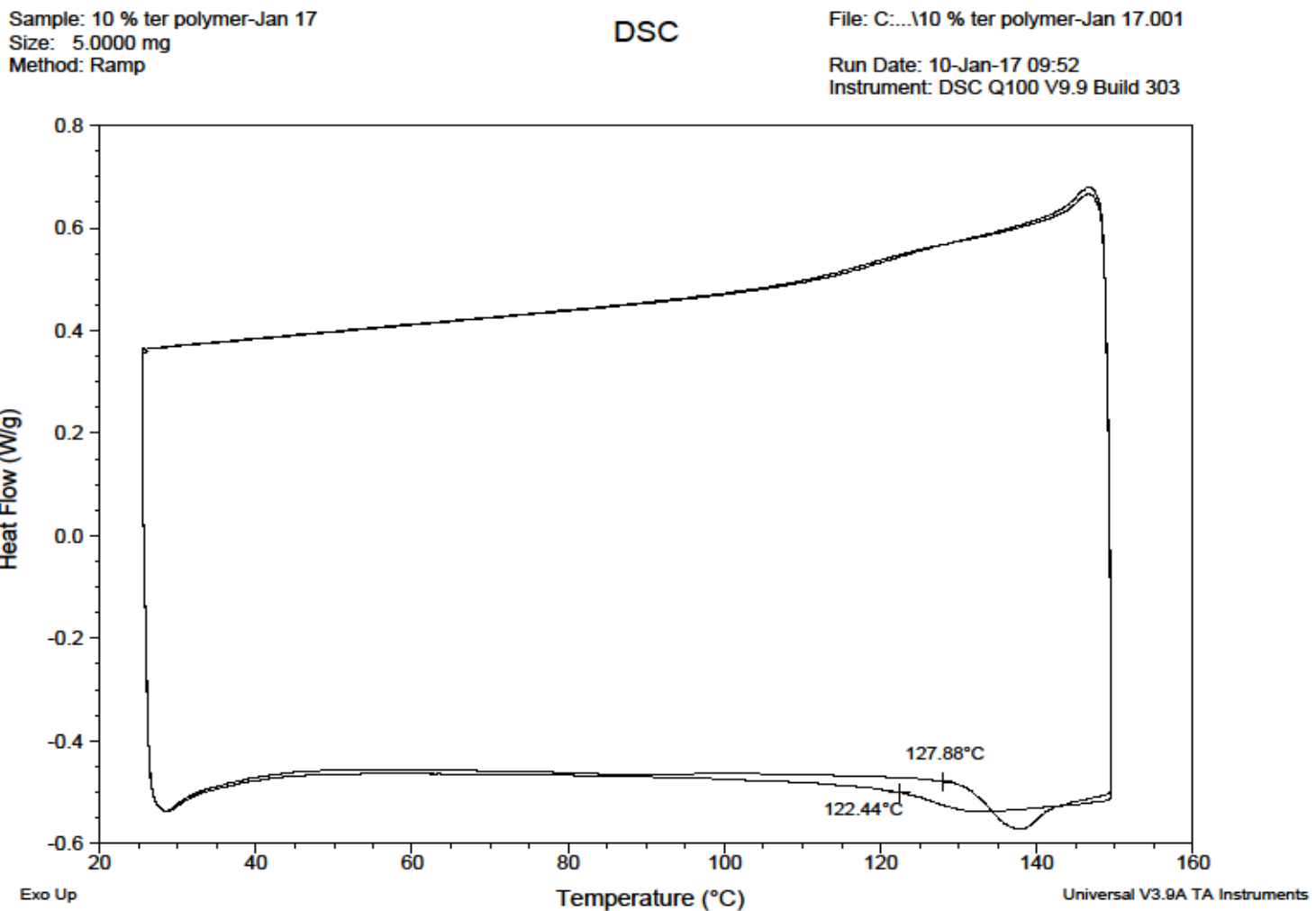

Figure S 3.25. DSC trace of $10 \mathrm{~mol} \%$ terpolymer (P13)

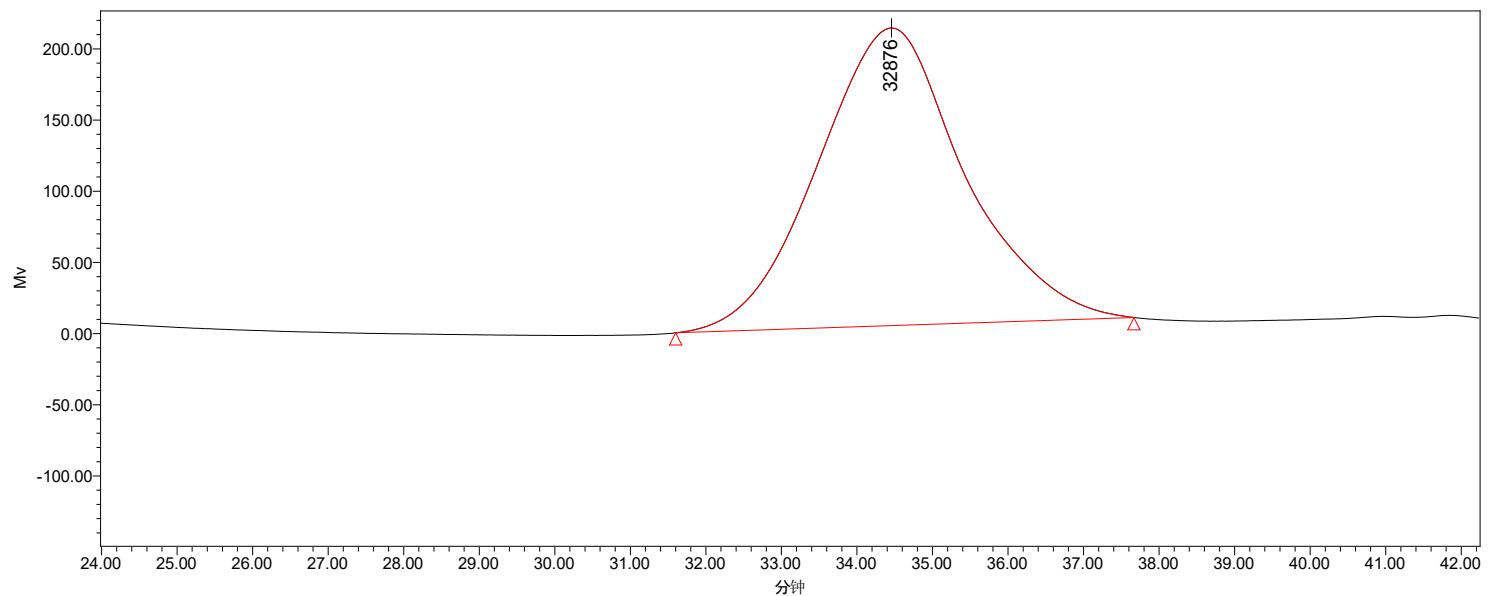

$\begin{array}{lllllll}\text { Mn } & \text { Mw } & \text { MP } & \text { Mv } & & \mathrm{Mz} & \mathrm{Mz}+1\end{array}$

25428. $35 \quad 41125.79 \quad 32875.71 \quad 38254.35 \quad 64285.55 \quad 94174.17$

Figure S3.26. GPC of $10 \mathrm{~mol} \%$ terpolymer (P13) 


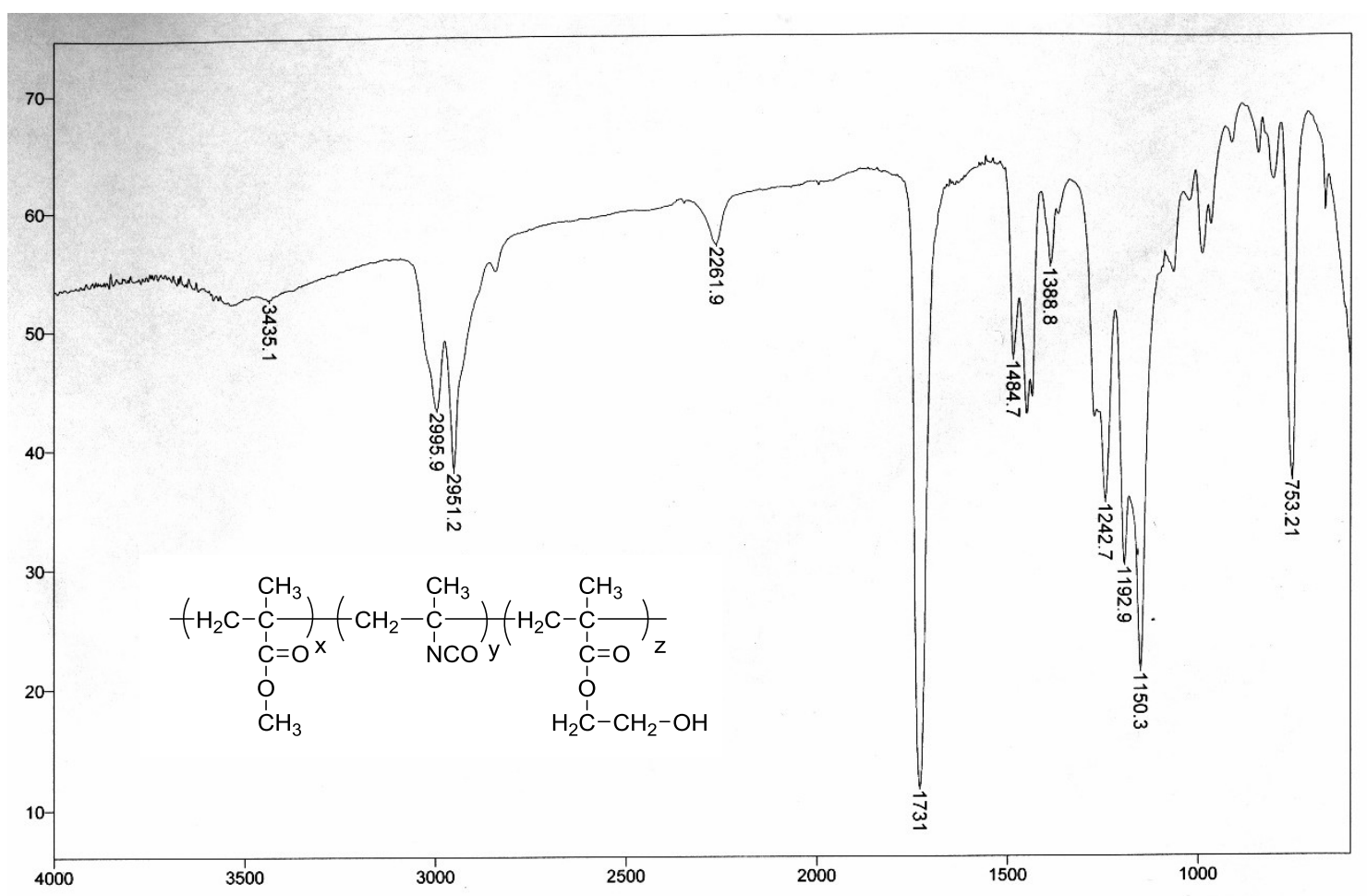

Figure S3.27. IR spectrum of the thermal conversions of $5 \mathrm{~mol} \%$ terpolymer to NCO-pendent polymer at $110^{\circ} \mathrm{C}(\mathrm{NaCl}$ plate $)$ 


\section{Appendix C Supporting Information for Chapter 4}

\section{Direct Detection of Ultralow Trace Amount of Isocyanates in Air Using Fluorescent}

\section{Conjugated Polymer}

\section{The permission letter from the Royal Society of Chemistry (RSC) for copying of Chapter 4}

and 5 is as below

Dear Khama

The Royal Society of Chemistry (RSC) hereby grants permission for the use of your paper(s) specified below in the printed and microfilm version of your thesis. You may also make available the PDF version of your paper(s) that the RSC sent to the corresponding author(s) of your paper(s) upon publication of the paper(s) in the following ways: in your thesis via any website that your university may have for the deposition of theses, via your university's Intranet or via your own personal website. We are however unable to grant you permission to include the PDF version of the paper(s) on its own in your institutional repository. The Royal Society of Chemistry is a signatory to the STM Guidelines on Permissions (available on request).

You may include it as a word document as long as it is fully acknowledged.

Please note that if the material specified below or any part of it appears with credit or acknowledgement to a third party then you must also secure permission from that third party before reproducing that material. 
Please ensure that the thesis states the following: Reproduced by permission of The Royal Society of Chemistry and include a link to the paper on the Royal Society of Chemistry's website.

Please ensure that your co-authors are aware that you are including the paper in your thesis.

Regards

Gill Cockhead

Publishing Contracts \& Copyright Executive

Royal Society of Chemistry, Thomas Graham House, Science Park, Milton Road, Cambridge, CB4 0WF, UK Tel +44 (0) 1223432134 


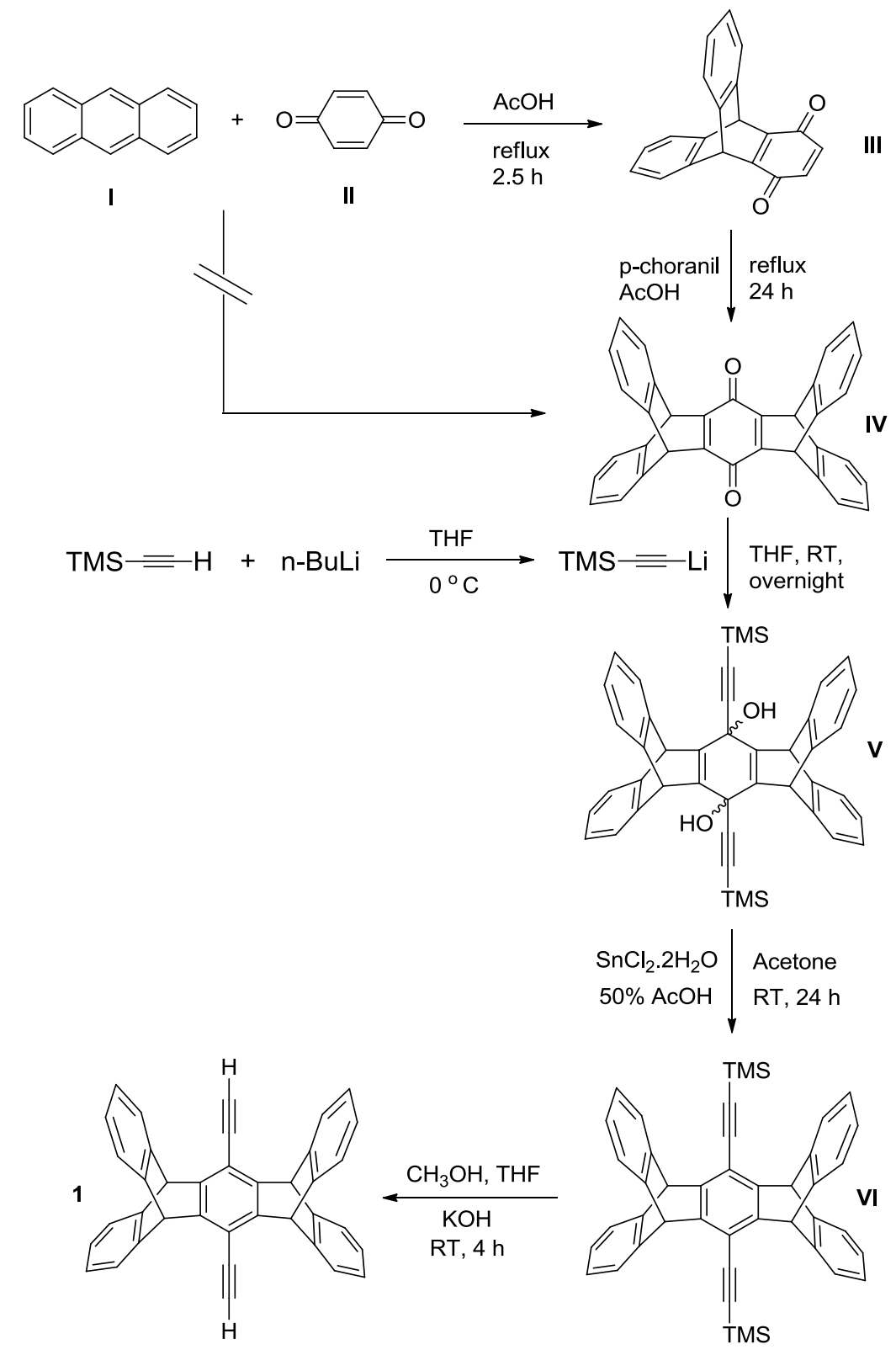

Scheme S1. The synthesis pathway of monomer 1 


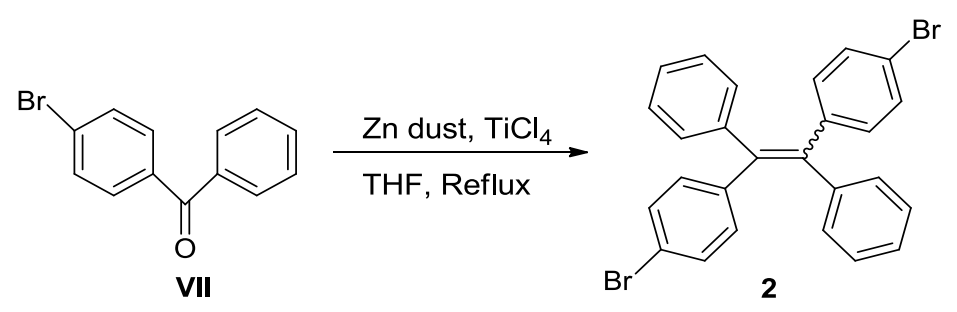

Scheme S2. The synthesis pathway of monomer 2

9,10-Dihydro-9,10-o-benzenoanthracene-1,4-dione (III). Under an argon atmosphere in a twoneck flask, a mixture of anthracene (I) $(10.0 \mathrm{~g}, 56.0 \mathrm{mmol})$ and $p$-benzoquinone (II) (20.27 g, $112.0 \mathrm{mmol}$ ) in $70 \mathrm{~mL}$ acetic acid was refluxed with vigorous stirring for $2.5 \mathrm{~h}$. The reaction progress was monitored by TLC (Hexane: Acetone, 3:1). The resulting reaction mixture was poured into water and after filtering the crude was washed with hot water. The crude product was then purified by silica gel column chromatography using hexane as a first eluting solvent and then chloroform as a second one. Compound III (11.9432 g, 72.0\%) was then obtained as yellow solid after removing the solvent. Product III (m.p $293.0{ }^{0} \mathrm{C}$, lit ${ }^{5} \mathrm{~m} . \mathrm{p}$ 292-296 $\left.{ }^{0} \mathrm{C}\right):{ }^{1} \mathrm{H}$ NMR $(300$ $\left.\mathrm{MHz}, \mathrm{CDCl}_{3}, \mathrm{ppm}\right): \delta 7.41(4 \mathrm{H}, \mathrm{m}), 7.06(4 \mathrm{H}, \mathrm{m}), 6.52(2 \mathrm{H}, \mathrm{s}), 5.81(2 \mathrm{H}, \mathrm{s}) .{ }^{13} \mathrm{C} \mathrm{NMR}(75 \mathrm{MHz}$, $\left.\mathrm{CDCl}_{3}, \mathrm{ppm}\right): \delta 183.54,151.93,150.96,143.58,135.40,125.98,125.48,124.44,124.26,47.36$. IR (NaCl Plate, $\left.\mathrm{cm}^{-1}\right): 2980\left(v_{\mathrm{CH}}\right)$.

5,6,7,12,13,14-Hexahydro-5,14:7,12-bis(o-benzene)pentacene-6,13-dione (IV). Under an atmosphere of argon in a two-neck flask a mixture of compound III (11.0 g, $37.16 \mathrm{mmol})$, anthracene I (6.62 g, $37.16 \mathrm{mmol})$ and $p$-chloranil $(9.27 \mathrm{~g}, 37.16 \mathrm{mmol})$ in $500 \mathrm{~mL}$ acetic acid were refluxed for $24 \mathrm{~h}$. The progress of the reaction was monitored by TLC (Hexane: Acetone, $3: 1 \mathrm{v} / \mathrm{v})$. The resulting mixture was then cooled to room temperature and the filtered precipitate was washed with ether and dried in vacuum oven to give $16.61 \mathrm{~g}(92.0 \%)$ of compound IV as a yellow solid; m.p $>350{ }^{\circ} \mathrm{C}$, lit. ${ }^{5} \mathrm{~m} . \mathrm{p}>370{ }^{\circ} \mathrm{C} .{ }^{1} \mathrm{H} \mathrm{NMR}\left(300 \mathrm{MHz}, \mathrm{CDCl}_{3}, \mathrm{ppm}\right): \delta 7.37(8 \mathrm{H}$, 
m), $6.99(8 \mathrm{H}, \mathrm{m}), 5.77(4 \mathrm{H}, \mathrm{s}) .{ }^{13} \mathrm{C} \mathrm{NMR}\left(75 \mathrm{MHz}, \mathrm{CDCl}_{3}, \mathrm{ppm}\right): \delta 180.00,150.96,143.64$, $125.48,124.26,47.36$.

Compound V. In a flame dried two-necks round bottom flask, under an argon atmosphere I equivalent of n-butyllithium $(33.34 \mathrm{~mL}, 82.55 \mathrm{mmol})$ in hexane was added dropwise to a solution of (trimethylsilyl)acetylene $(11.64 \mathrm{~mL}, 82.55 \mathrm{mmol})$ in $80 \mathrm{~mL} \mathrm{THF}$ at $0{ }^{\circ} \mathrm{C}$. Before it was transferred to a solution of compound IV $(16.0 \mathrm{~g}, 33.02 \mathrm{mmol})$ in $400 \mathrm{~mL}$ THF at $0{ }^{0} \mathrm{C}$ the mixture was kept at $0{ }^{\circ} \mathrm{C}$ for another $50 \mathrm{~min}$. The reaction mixture was then warmed to room temperature. The reaction was monitored by TLC (Hexane: Acetone, 3:1). After overnight stirring $20 \mathrm{~mL} 10 \% \mathrm{HCl}$ solution was added to the reaction mixture and the mixture was then extracted with chloroform. After removing the solvent hexane was added to the residue. Compound V (20.15 g, 89.62\%) was collected by filtration as a mixture of cis and trans isomers. ${ }^{1} \mathrm{H}$ NMR (300 MHz, $\left.\mathrm{CDCl}_{3}, \mathrm{ppm}\right): 7.35$ (8H, m), $6.92(8 \mathrm{H}, \mathrm{m}), 5.50(4 \mathrm{H}, \mathrm{s}), 0.19$ (18H, s). IR (KBr Pellet, cm $\left.{ }^{-1}\right):$ 3498, 3257, 3068, 3019, 2959, 2897, 2173.

Compound VI. In a solution of compound V (5.0 g, $28.0 \mathrm{mmol})$ in $100 \mathrm{~mL}$ of acetone, a solution of tin (II) chloride dehydrate $(4.13 \mathrm{~g}, 18.25 \mathrm{mmol})$ in $50 \%$ of acetic acid $(50.0 \mathrm{~mL})$ was added dropwise. This mixture was stirred at room temperature for another $24 \mathrm{~h}$ and the reaction progress was monitored by TLC (Hexane: Acetone, 3:1). After filtering the resulting solid it was dissolved in chloroform and washed with water and then sodium bicarbonate solution twice. The solvent was removed by the rotor-evaporation and the residue was precipitated in hexane. The resulting solid was dried in vacuum oven and collected as a white solid (3.50 g, 73\%), m.p 417 ${ }^{\circ} \mathrm{C}$, lit. ${ }^{1}$ obtained m.p $419{ }^{\circ} \mathrm{C} .{ }^{1} \mathrm{H}$ NMR (300 MHz, $\left.\mathrm{CDCl}_{3}, \mathrm{ppm}\right): 7.35$ (8H, m), 6.96 (8H, m), $5.79(4 \mathrm{H}, \mathrm{s}), 0.50(18 \mathrm{H}, \mathrm{s}) .{ }^{13} \mathrm{C} \mathrm{NMR}\left(100 \mathrm{MHz}, \mathrm{CDCl}_{3}, \mathrm{ppm}\right): \delta 144.86,144.05,129.19$, 125.20, 123.77, 114.82, 102.48, 100.60, 52.07, 29.69, 1.01. IR (NaCl Plate, $\left.\mathrm{cm}^{-1}\right): 2957\left(v_{\mathrm{CH}}\right)$. 
Compound 1. In a solution of compound VI (2.80 g, $15.71 \mathrm{mmol})$ in THF (30 mL) and $\mathrm{MeOH}$ $(30 \mathrm{~mL})$, a solution of $\mathrm{KOH}\left(2.0 \mathrm{~g}\right.$ in $5 \mathrm{~mL}$ of $\left.\mathrm{H}_{2} \mathrm{O}\right)$ was added and the mixture was stirred at room temperature for $4 \mathrm{~h}$. The progress of the reaction was monitored by TLC (Hexane: Dichloromethane, 3:1). The resulting solid product was filtered and washed with water and hexane. After dried in vacuum oven compound $\mathbf{1}$ was obtained as white solid powder (2.05 g, 95\%). m.p $437{ }^{\circ} \mathrm{C}$, lit. ${ }^{1}$ m.p $439.5^{\circ} \mathrm{C} .{ }^{1} \mathrm{H}$ NMR (300 MHz, $\left.\mathrm{CDCl}_{3}, \mathrm{ppm}\right): 7.37$ (8H, m), $6.96(8 \mathrm{H}$, m), $5.84(4 \mathrm{H}, \mathrm{s}), 3.71(2 \mathrm{H}, \mathrm{s}) .{ }^{13} \mathrm{C} \mathrm{NMR}\left(100 \mathrm{MHz}, \mathrm{C}_{2} \mathrm{D}_{2} \mathrm{Cl}_{4} \mathrm{ppm}\right): \delta 144.06,143.95,124.90$, 123.54, 113.57, 84.84, 78.03, 51.27. IR (KBr Pellet, $\left.\mathrm{cm}^{-1}\right): 3303\left(v_{\mathrm{C} \equiv \mathrm{C}}\right)$.

1,2-bis (4-bromophenyl)-1,2-diphenylethene (2). In a flame-dried three-neck flask under argon atmosphere, an ice-cooled $\left(-5^{\circ} \mathrm{C}\right)$ suspension of zinc powder $(12.50 \mathrm{~g}, 191.19 \mathrm{mmol})$ in THF $(240 \mathrm{~mL})$ was prepared and titanium tetrachloride $(20.00 \mathrm{~mL}, 181.81 \mathrm{mmol})$ was slowly added to the suspension. The resulting mixture was refluxed for $4 \mathrm{~h}$. After cooling to room temperature, a solution of 4-bromobenzophenone $(10.00 \mathrm{~g}, 38.30 \mathrm{mmol})$ in THF $(50 \mathrm{~mL})$ was slowly added to the mixture, and the mixture was then refluxed overnight. After cooling to room temperature, the mixture was diluted with saturated aqueous sodium hydrogen carbonate solution (until no bubble come out) and dichloromethane and stirred for $5 \mathrm{~h}$. The mixture was filtered through a Celite pad, and the filtrate was separated into organic and aqueous layers. The aqueous layer was extracted thrice with dichloromethane, and the combined organic layer was dried over $\mathrm{MgSO}_{4}$ and the solvent was evaporated under the reduced pressure. The resulting white crude product was purified by silica gel column chromatography using hexane as eluting solvent. Compound 2 was obtained as white powder $(8.50 \mathrm{~g}, 90.55 \%$ yield $)$ after removing the solvent. Compound 2 was further purified by multiple recrystallizations in ethanol: toluene (3:2) and hexane: ethyl 
acetate (8:2) and obtained as white crystal. ${ }^{1} \mathrm{H}$ NMR (300 MHz, $\left.\mathrm{CDCl}_{3}, \mathrm{ppm}\right): \delta 7.26(4 \mathrm{H}, \mathrm{m})$, $7.15(6 \mathrm{H}, \mathrm{m}), 7.01(4 \mathrm{H}, \mathrm{m}), 6.90(4 \mathrm{H}, \mathrm{m}) .{ }^{13} \mathrm{C} \mathrm{NMR}\left(100 \mathrm{MHz}, \mathrm{CDCl}_{3}\right): 142.93,142.83,142.39$, $142.29,140.31,132.87,131.21,131.11,130.91,128.02,127.82,126.95,126.83,120.80,120.66$. EI MS calculated for $\left[\mathrm{M}^{+}\right]$490.23, found 490. IR $\left(\mathrm{KBr}\right.$ Pellet, $\left.\mathrm{cm}^{-1}\right): 1640\left(v_{\mathrm{C}=\mathrm{C}}\right)$.

Polymer P1. In a flame dried two-necks $25 \mathrm{~mL}$ round-bottom flask, under an argon atmosphere monomer $1(0.2513 \mathrm{~g}, 0.50 \mathrm{mmol})$ and dibromo-TPE $(0.2451 \mathrm{~g}, 0.50 \mathrm{mmol})$ was added to diisopropylaminelanhydrous toluene $(2.8: 4.2,7 \mathrm{~mL})$. After degassed and purged with argon for $30 \mathrm{~min}, \mathrm{Pd}\left(\mathrm{PPh}_{3}\right)_{4}(0.030 \mathrm{~g}, 5 \mathrm{~mol} \%)$ and $\mathrm{CuI}(0.0047 \mathrm{~g}, 5 \mathrm{~mol} \%)$ were added. The mixture was heated at $90{ }^{\circ} \mathrm{C}$ for $48 \mathrm{~h}$ under argon. The reaction mixture was then dissolved in $\mathrm{CHCl}_{3}$ and washed with aqueous $\mathrm{NH}_{4} \mathrm{Cl}$ solution and dried over anhydrous $\mathrm{MgSO}$. The solvent was removed by rotor-evaporator and the residue was dissolved into $\mathrm{CHCl}_{3}$ and precipitated with $\mathrm{MeOH}$ three times. After drying under vacuum at $80{ }^{\circ} \mathrm{C}$ for $5 \mathrm{~h}$, the polymers were obtained ( $0.3019 \mathrm{~g}, 71 \%$ of yield) as greenish-yellow powder. 


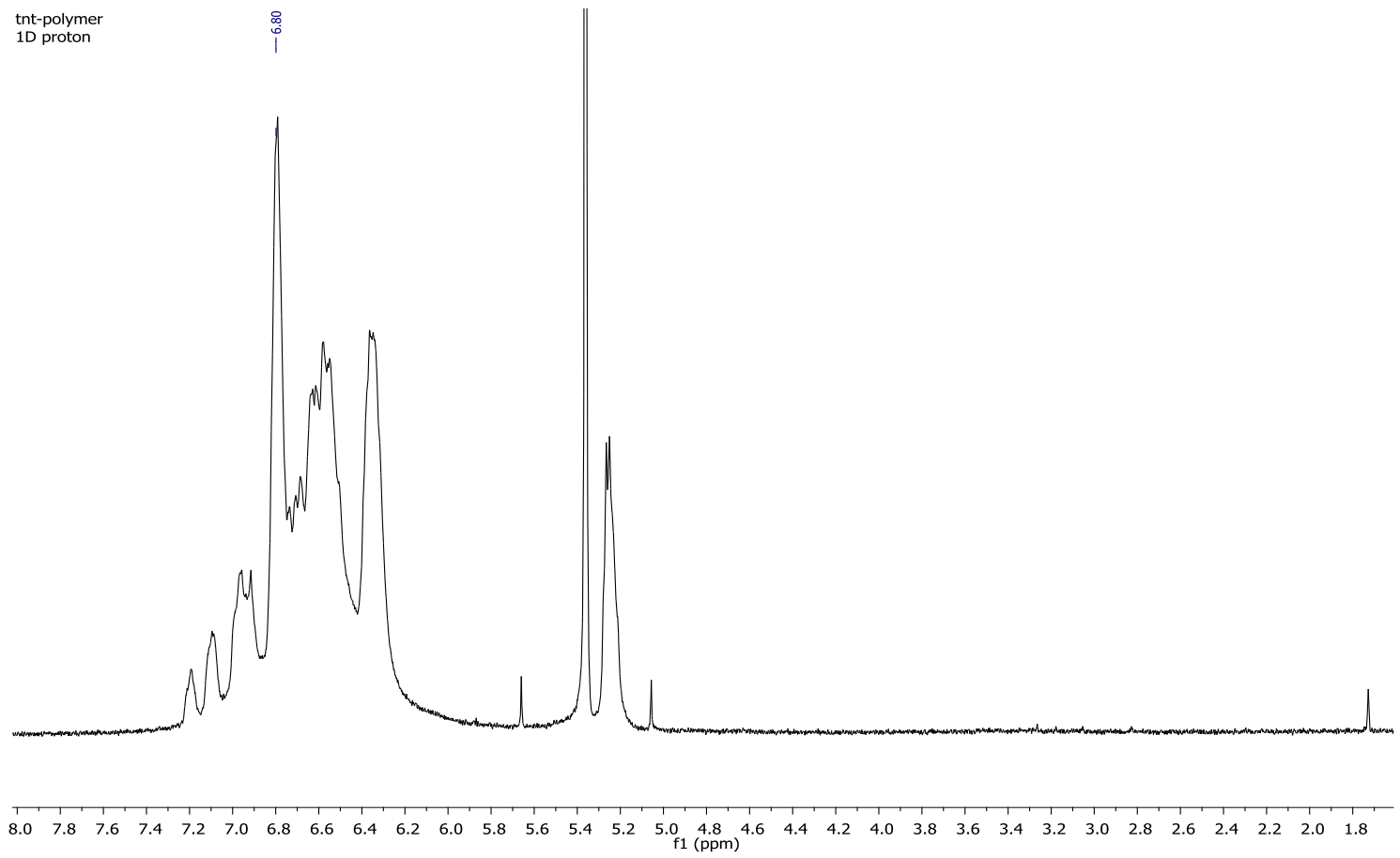

Figure S1. The ${ }^{1} \mathrm{H}$ NMR spectrum of polymer $\mathbf{P 1}\left(300 \mathrm{MHz}, \mathrm{C}_{2} \mathrm{D}_{2} \mathrm{Cl}_{4}\right.$ )

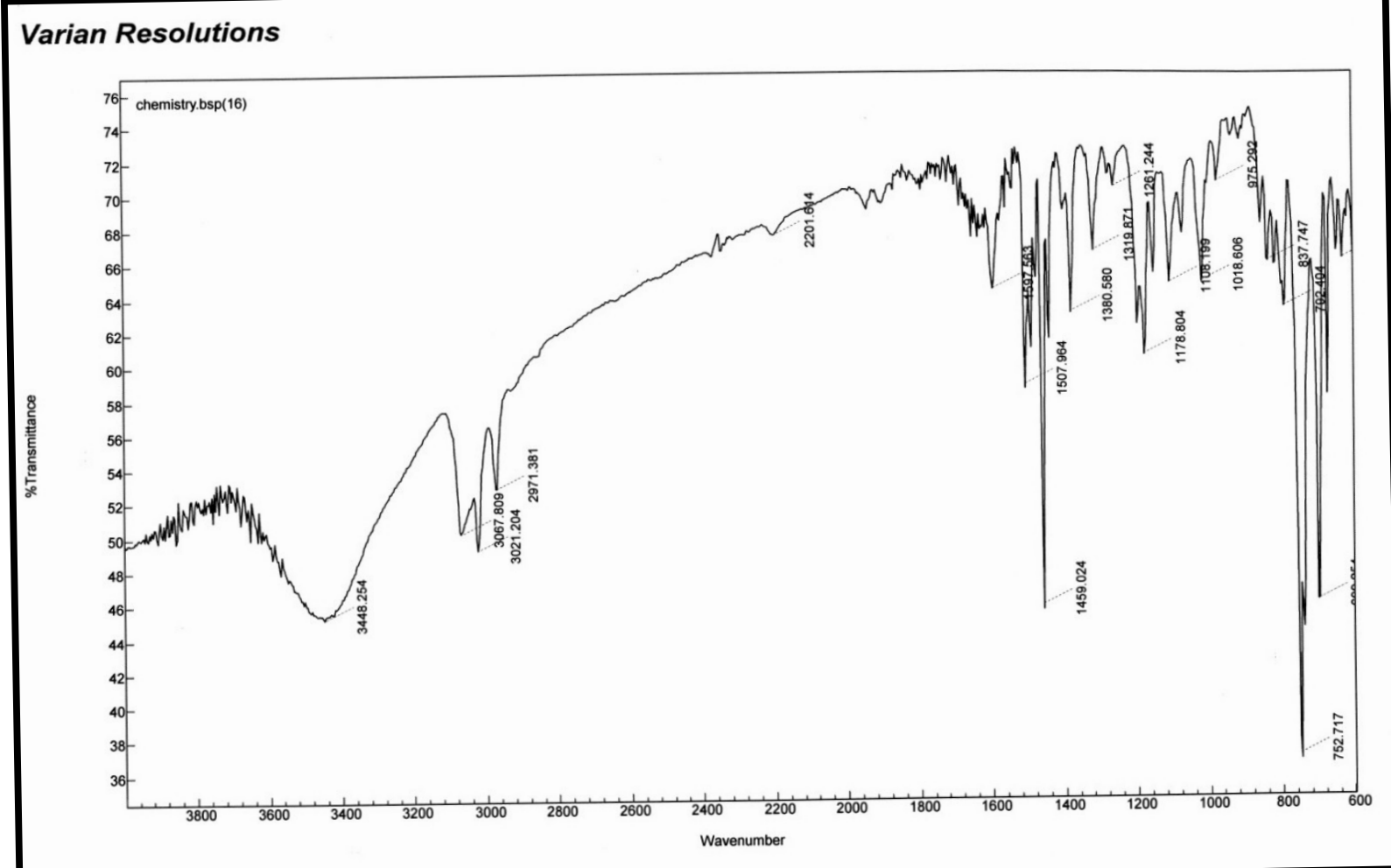

Figure S2. The IR spectrum of polymer P1 (KBr pellet). 


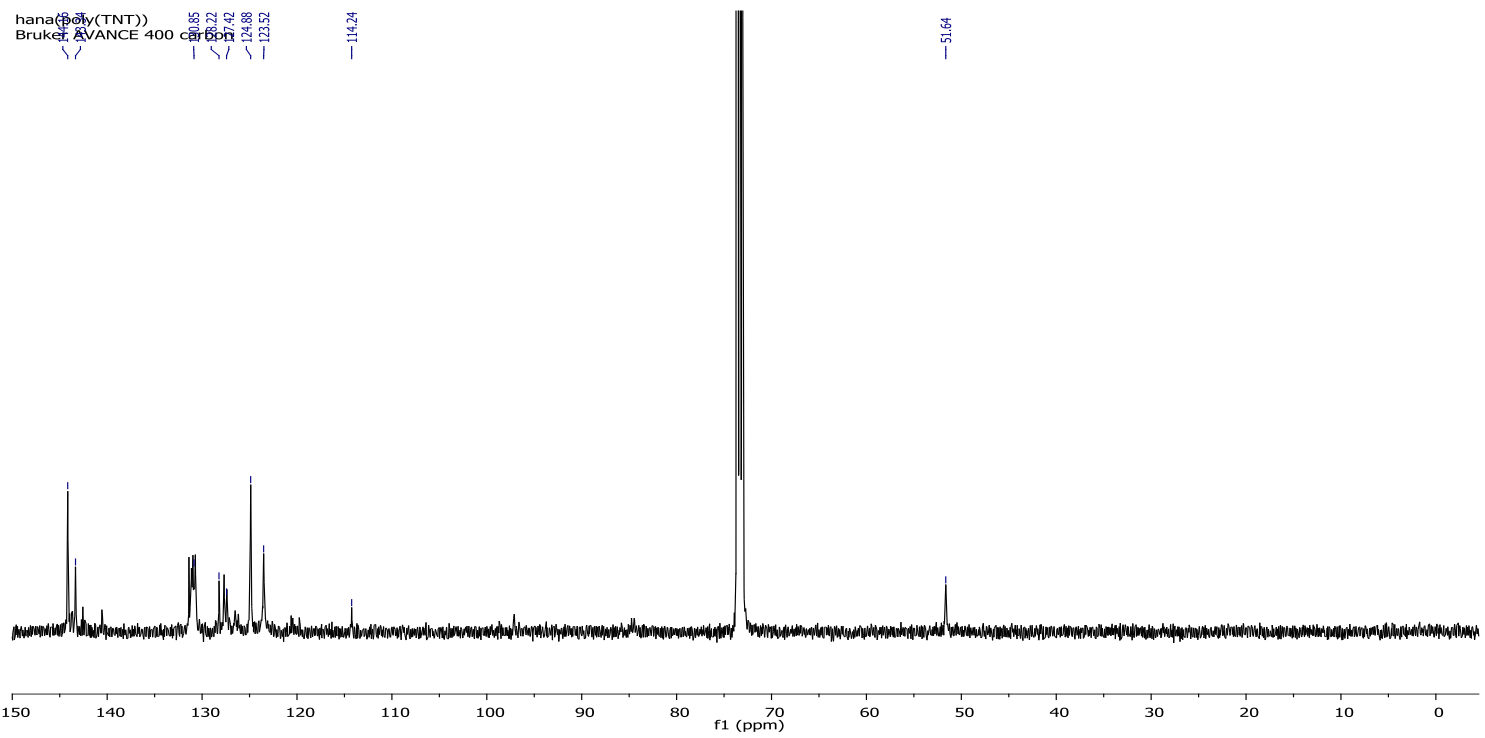

Figure S3. The ${ }^{13} \mathrm{C}$ NMR spectrum of polymer $\mathbf{P 1}\left(100 \mathrm{MHz}, \mathrm{C}_{2} \mathrm{D}_{2} \mathrm{Cl}_{4}\right)$

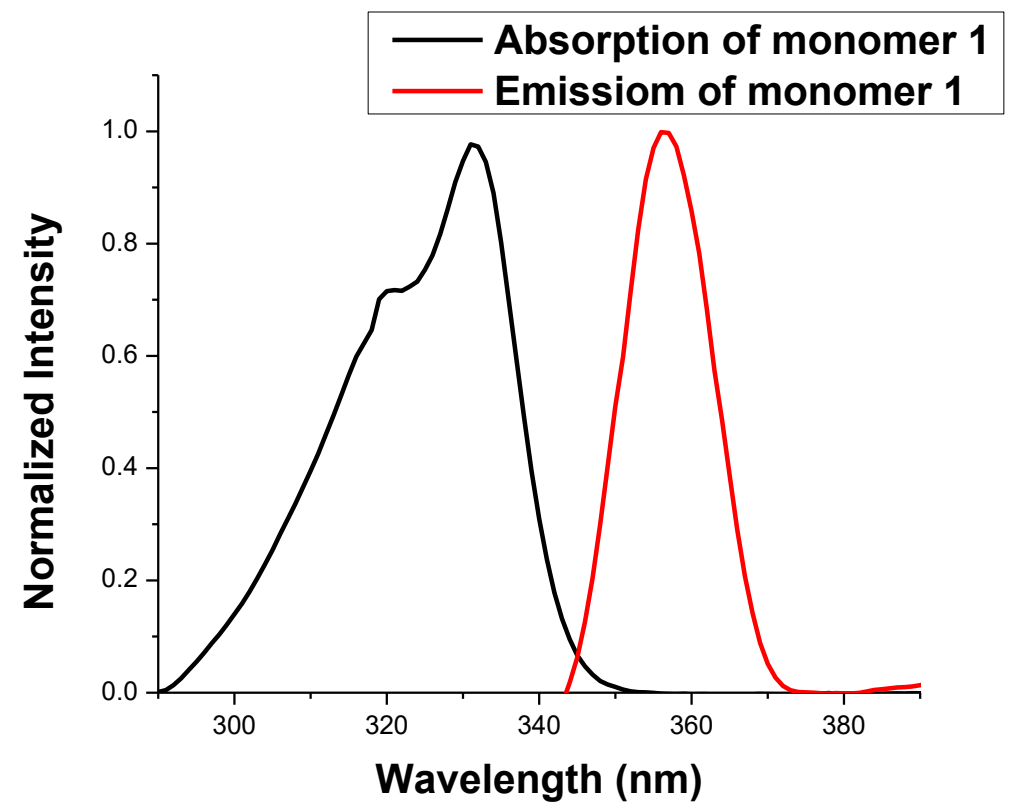

Figure S4. Normalized absorption and emission spectra of monomer 1 in 1,1,2,2tetrachloroethane. 


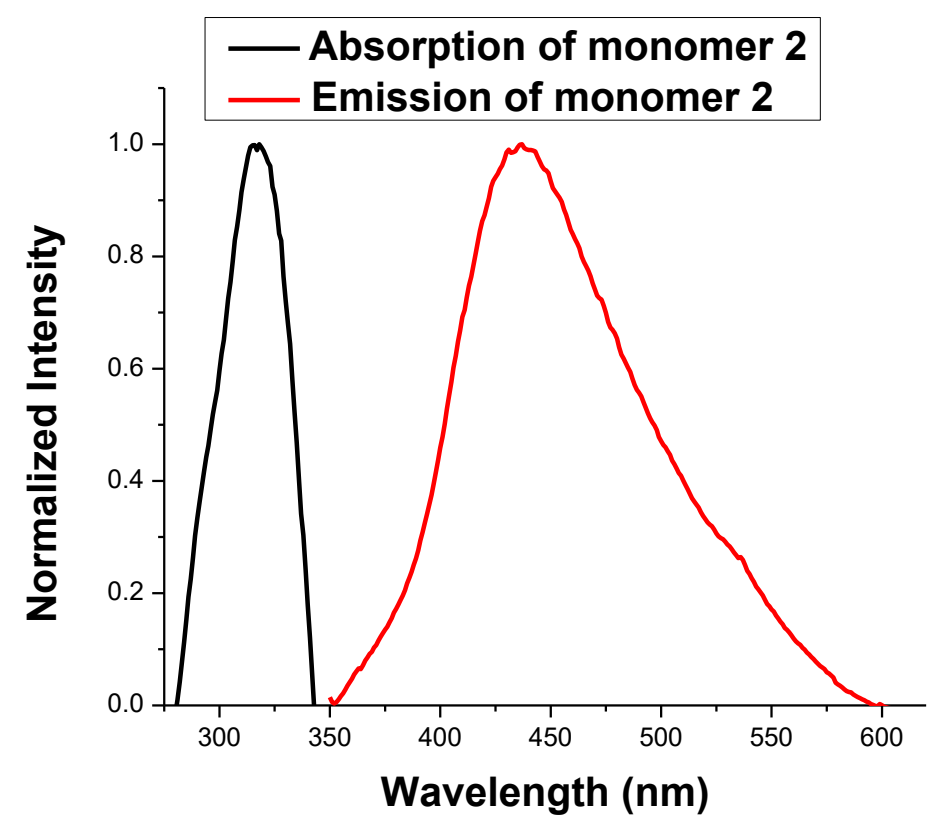

Figure S5. Normalized absorption and emission spectra of monomer 2 in $\mathrm{CHCl}_{3}$

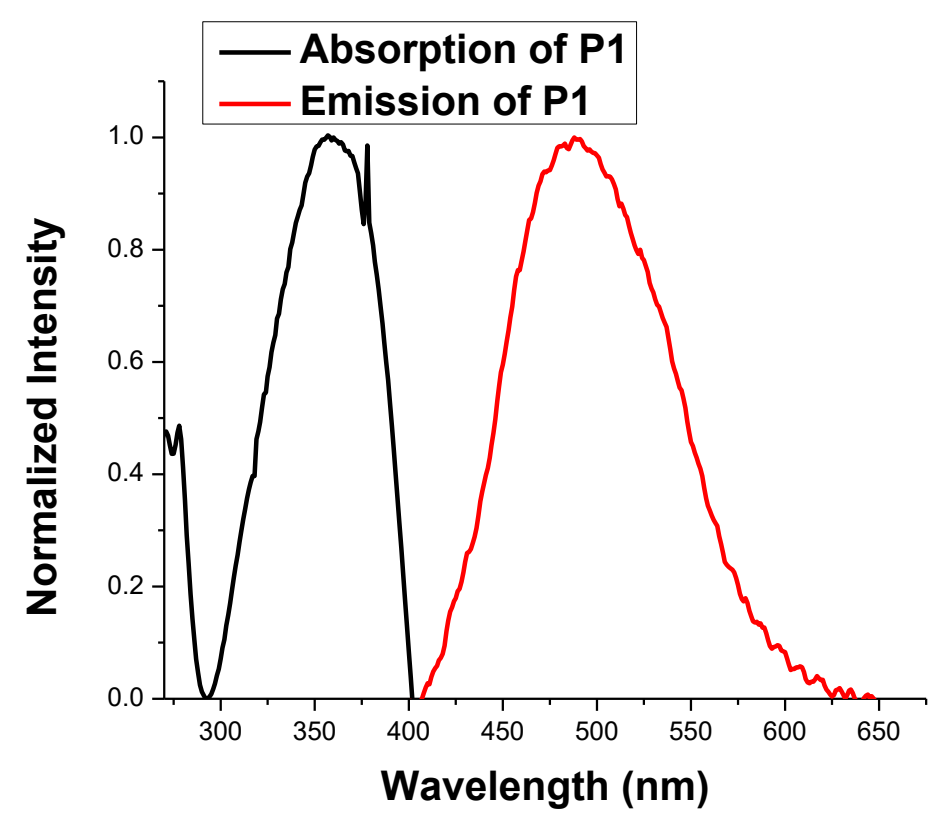




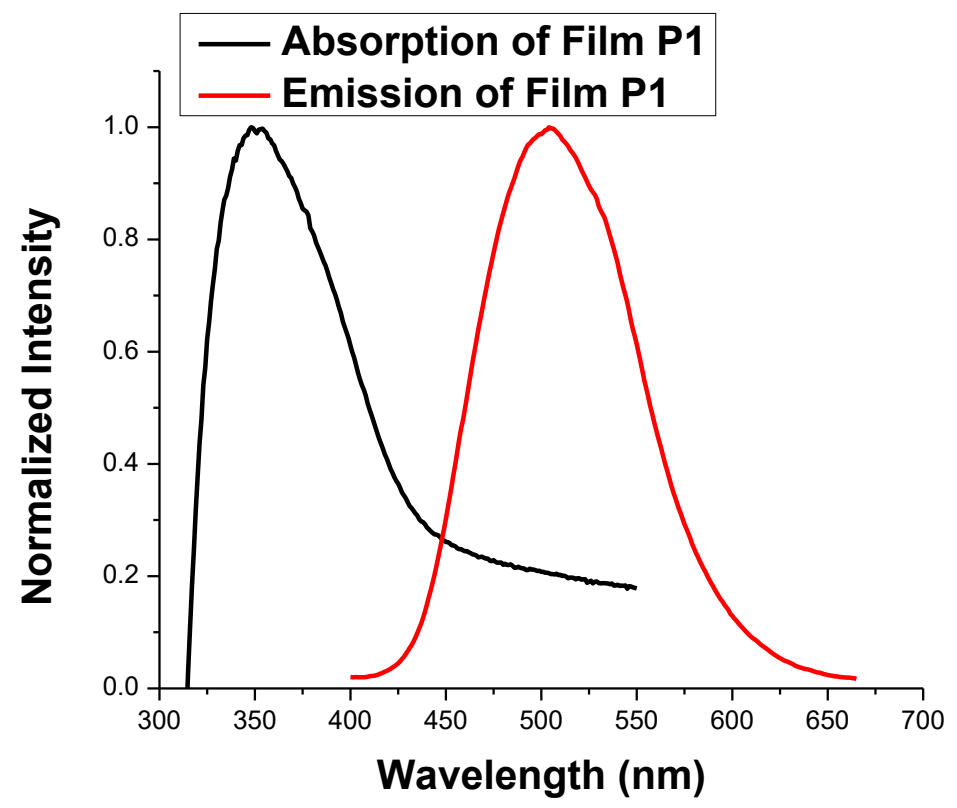

Figure S6. Normalized absorption and emission of polymer P1 in THF solution (top) and in film (bottom).

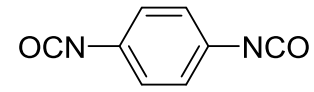

p-phenylene diisocyanate PPDI<smiles>O=Nc1ccc([N+](=O)[O-])cc1</smiles>

4-Nitrophenyl isocyanate $\mathrm{NO}_{2} \mathrm{PI}$<smiles>O=Nc1ccc(Cc2ccc(N=O)cc2)cc1</smiles>

4,4'-Methylenebis(phenyl isocyanate) Toluene 2,4-diisocyanate MDI

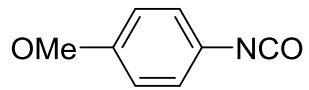

4-Methoxyphenyl isocyanate MeOPI<smiles>Cc1ccc([N+](=O)[O-])cc1[N+](=O)[O-]</smiles>
TDI Phenyl isocyanate

PI<smiles>CC1(C)CC(CN=O)C([N+](=O)[O-])CC1(C)C</smiles>

Isophorone diisocyanate IPDI

Figure S7. Structures of target isocyanates 
Table S1. Vapor concentration of isocyanates ${ }^{1}$

\begin{tabular}{|l|l|l|l|l|}
\hline Isocyanate & $\begin{array}{l}\text { Vapor pressure } \\
(\mathrm{mmHg})\end{array}$ & $\begin{array}{l}\text { Vapor } \\
\text { concentration } \\
(\mathrm{pg} / \mathrm{mL})\end{array}$ & $\begin{array}{l}\text { Molarity of NCO } \\
\text { group10 } \\
(\mathrm{mol} / \mathrm{L})\end{array}$ & $\mathrm{Mw}(\mathrm{g} / \mathrm{mol})$ \\
\hline $\mathrm{PPDI}$ & $<6.0 \times 10^{-3}$ & 52.46 & 0.655 & 160.13 \\
$\mathrm{TDI}$ & $<0.01$ & 95.09 & 1.092 & 174.15 \\
$\mathrm{MDI}$ & $4.5 \times 10^{-6}$ & 0.062 & $4.9 \times 10^{-4}$ & 250.56 \\
$\mathrm{PI}$ & 1.4 & 9105.14 & 76.44 & 119.12 \\
$\mathrm{MeOPI}$ & 0.107 & 871.33 & 5.842 & 149.15 \\
$\mathrm{NO}_{2} \mathrm{PI}$ & $6.84 \times 10^{-3}$ & 61.29 & 37.35 & 164.12 \\
$\mathrm{HDI}$ & $5.26 \times 10^{-3}$ & 48.34 & 0.287 & 168.22 \\
IPDI & $3.0 \times 10^{-4}$ & 3.64 & 0.033 & 222.28 \\
\hline
\end{tabular}

\section{Film Preparation}

Films were coated on glass substrates by spin coating process. The polymer solution was spin-coated from 1,1,2,2 tetrachloroethane (TCE) solutions onto a glass plate (microscope slide, $20 \times 20 \times 1 \mathrm{~mm}$ ) and spun at $1000 \mathrm{rpm}$ by using a Chemat KW-4B spin-Coater. Then the films were dried for overnight at $80{ }^{\circ} \mathrm{C}$ under argon atmosphere. To prepare 4-80 nm thick films, 5-10 $\mathrm{mg}$ of polymer in $1.5 \mathrm{~mL}$ of solvent were used. The thickness of the films ranged from 4-80 nm, as measured using a Tencor Instrument Alpha-Step 200 surface profiler (uncertainty $\pm 0.01 \AA$ ) and by Atomic Force Microscopy (AFM) (uncertainty $\pm 0.2 \mathrm{~nm}$ ).

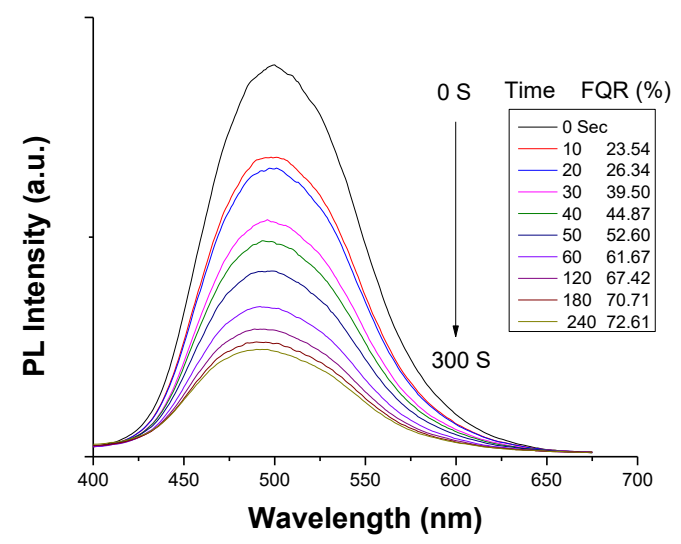

Figure S8. The time-dependent fluorescence intensity of P1 film ( $7 \mathrm{~nm})$ upon exposure to HDI vapor (room temperature) from $0 \mathrm{~s}$ to $240 \mathrm{~s}$. 


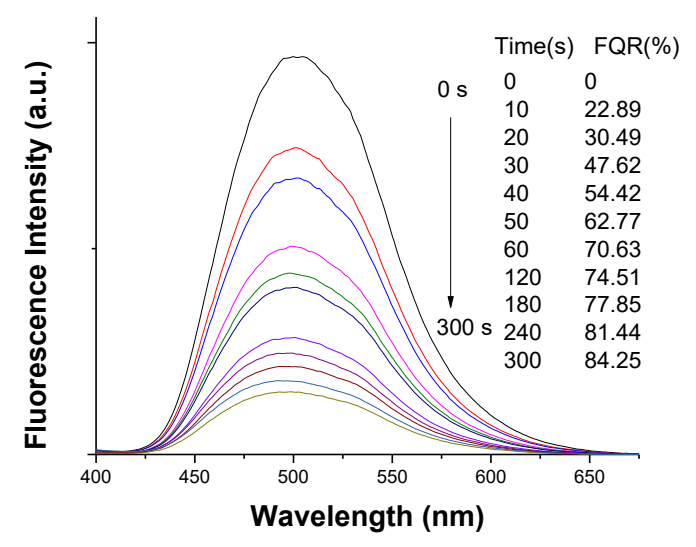

Figure S9. The time-dependent fluorescence intensity of P1 film (4 nm) upon exposure to PPDI vapor (room temperature) from 0 to $300 \mathrm{~s}$.

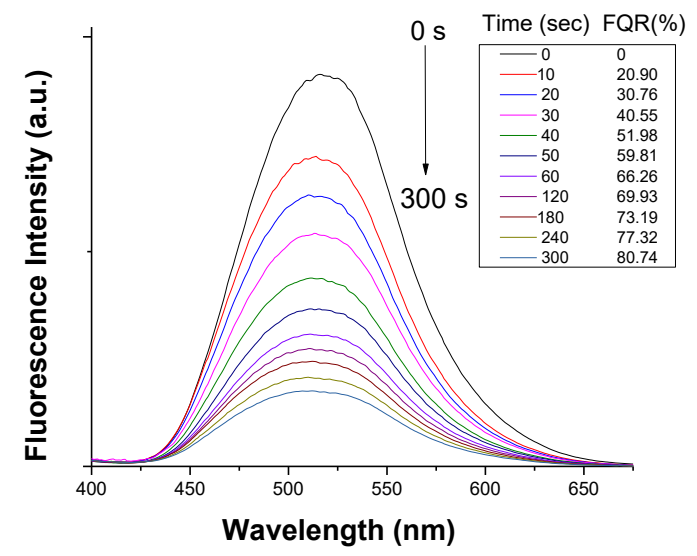

Figure S10. The time-dependent fluorescence intensity of P1 film (4nm) upon exposure to PI vapor (room temperature) from 0 to $300 \mathrm{~s}$. 


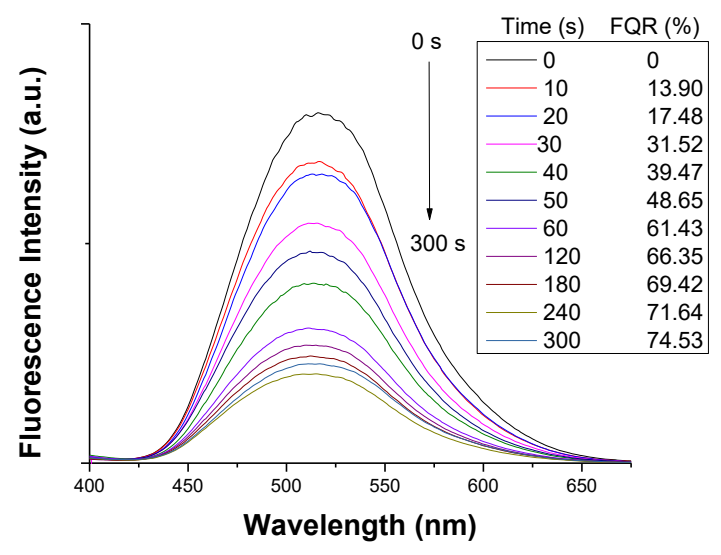

Figure S11. The time-dependent fluorescence intensity of P1 film (4nm) upon exposure to $\mathrm{NO}_{2} \mathrm{PI}$ vapor (room temperature) from 0 to $300 \mathrm{~s}$.

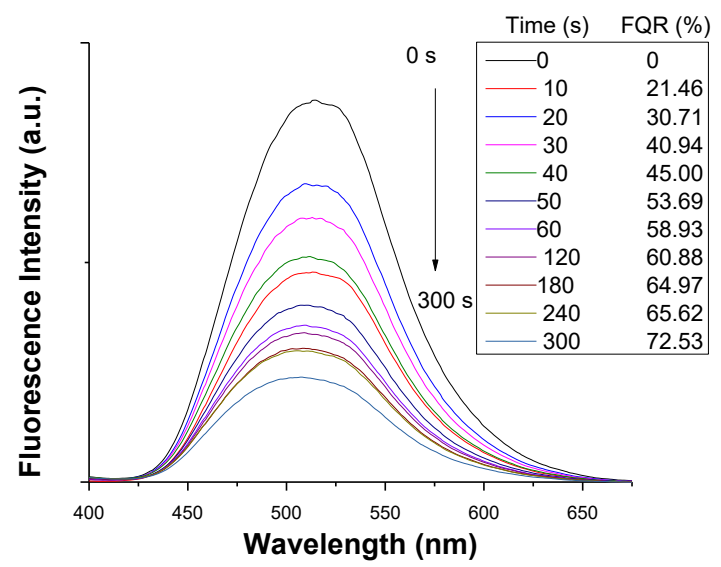

Figure S12. The time-dependent fluorescence intensity of P1 film (4nm) upon exposure to $\mathrm{MeOPI}$ vapor (room temperature) from 0 to $300 \mathrm{~s}$. 


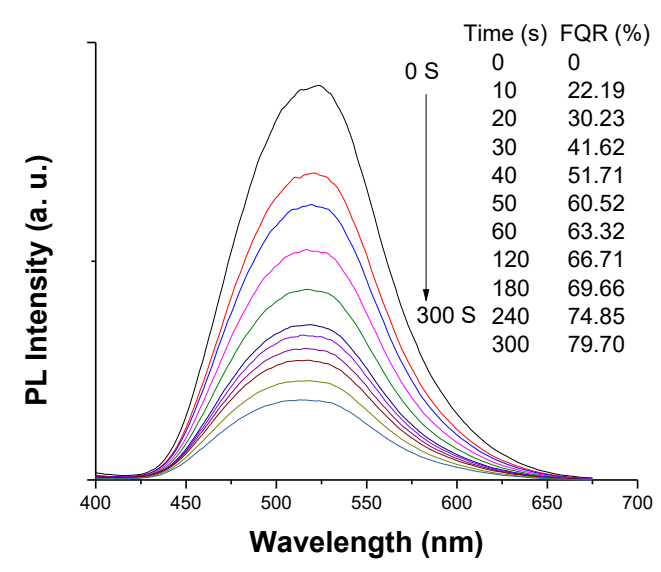

Figure S13. The time-dependent fluorescence intensity of P1 film (7nm) upon exposure to TDI vapor (room temperature) at $0,10,20,30,40,50,60,120,180,240$ and 300.
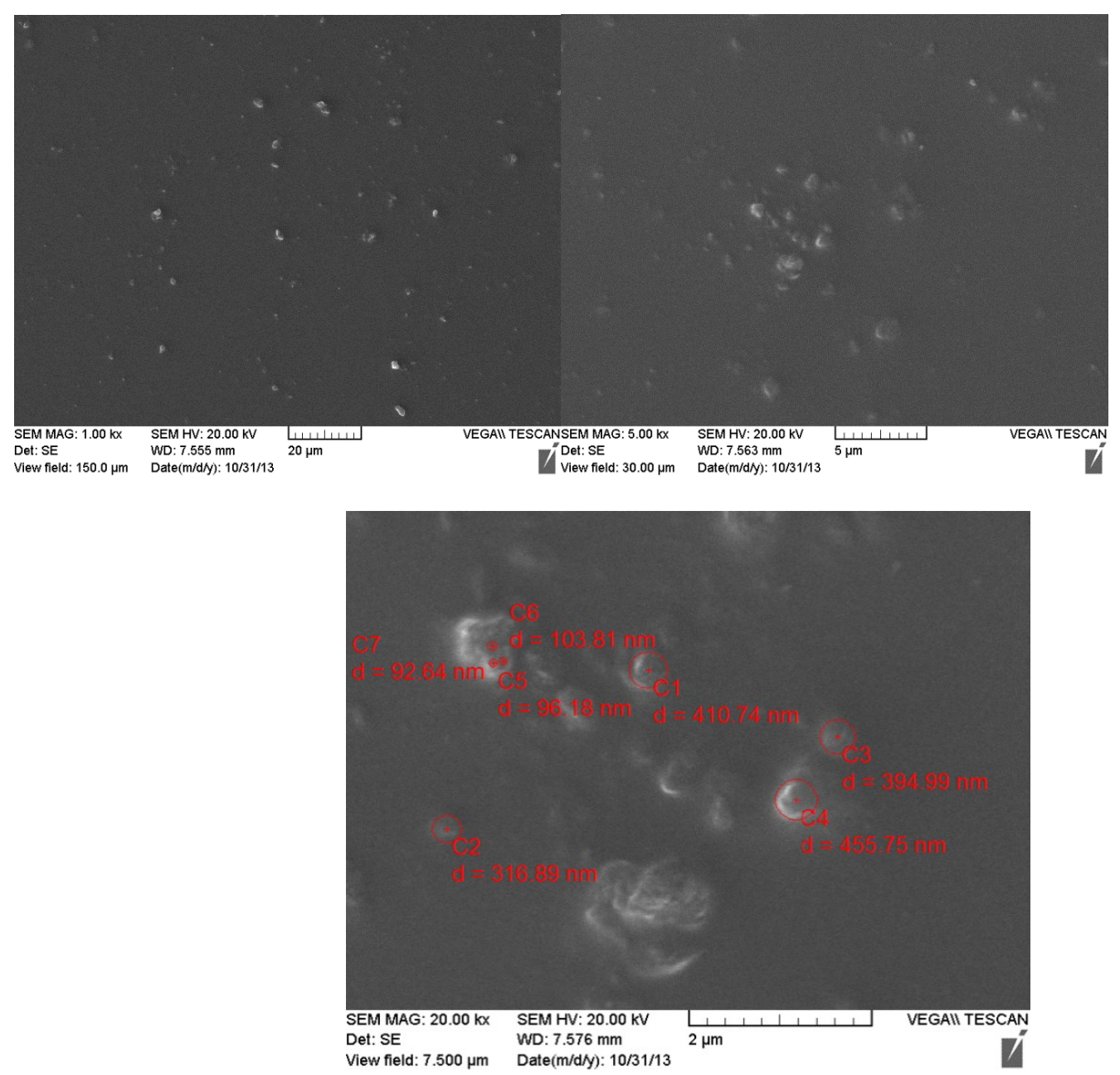

Figure S14. SEM images of Polymer P1 film on glass surface 


\section{REFERENCES}

(1) (a) Sigma-Aldrich Inc. (TDI, HDI, $\mathrm{NO}_{2} \mathrm{PI}$ ); (b) NIOSH (National Institute for Occupational Safety and Health) (MDI); (c) NTP (Normal Temperature and Pressure) (PPDI); (d) IPCS (International Programme on Chemical Safety) (PI, MeOPI and IPDI).

(2) (a) Québec, éditeur officiel, Réglementation sur la santé et sécurité autravail, décret 8852001, Québec, 2001, Canada. (b) American Conference of Governmental Industrial Hygienists, Threshold Limit Values for Chemical Substances and Physical Agents and Biological Exposure Indices, Cincinnati, OH, 2004. (c) National Institute for Occupational Safety and Health Alert, Publication no. 1996-111, Cincinnati, OH, 1996. (d) Swedish National Board of Occupational Safety and Health, Occupational exposure limit values. Arbetarskyddsstyrelsens fösfattningssamling, AFS 2000: 3, Liber, Stockholm, Sweden, 2000. (e) Code of Federal Regulation (CFR), Government Printing Office, office of the federal register, 29 CFR *1910.1000, Washington DC, 1992 


\section{Appendix D Supporting Information for Chapter 5}

Ultra-Sensitive Detection of Explosive in Solution and Film as well as the Development of

Thicker Film Effectiveness by Tetraphenylethene Moiety in AIE Active Fluorescent

\section{Conjugated Polymer}

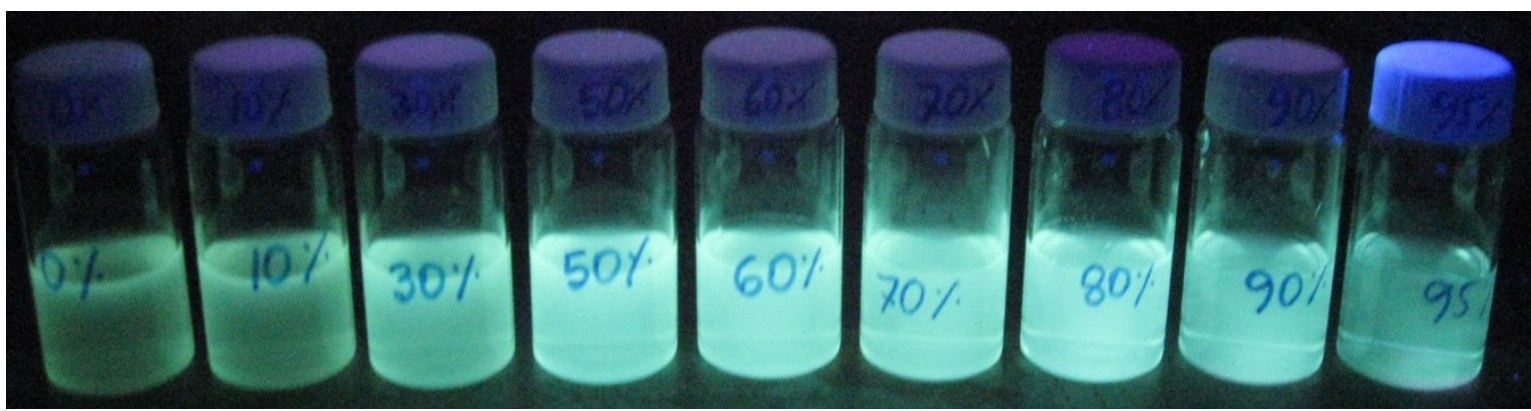

Fig. S1: Photographs of polymer $\mathbf{P 1}$ in $\mathrm{H}_{2} \mathrm{O} /$ THF mixtures with different fractions of water $\left(f_{\mathrm{w}}\right)$ taken under UV illumination.

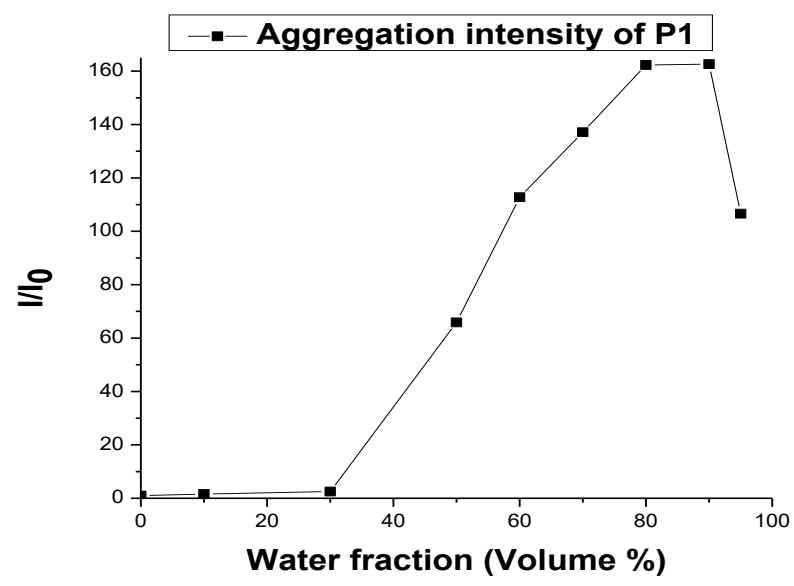

Fig. S2: Plot of $(\mathrm{I} / \mathrm{Io})$ values versus the compositions of the aqueous mixtures. $\mathrm{I}_{\mathrm{o}}$ is the emission intensity in pure THF solution. 


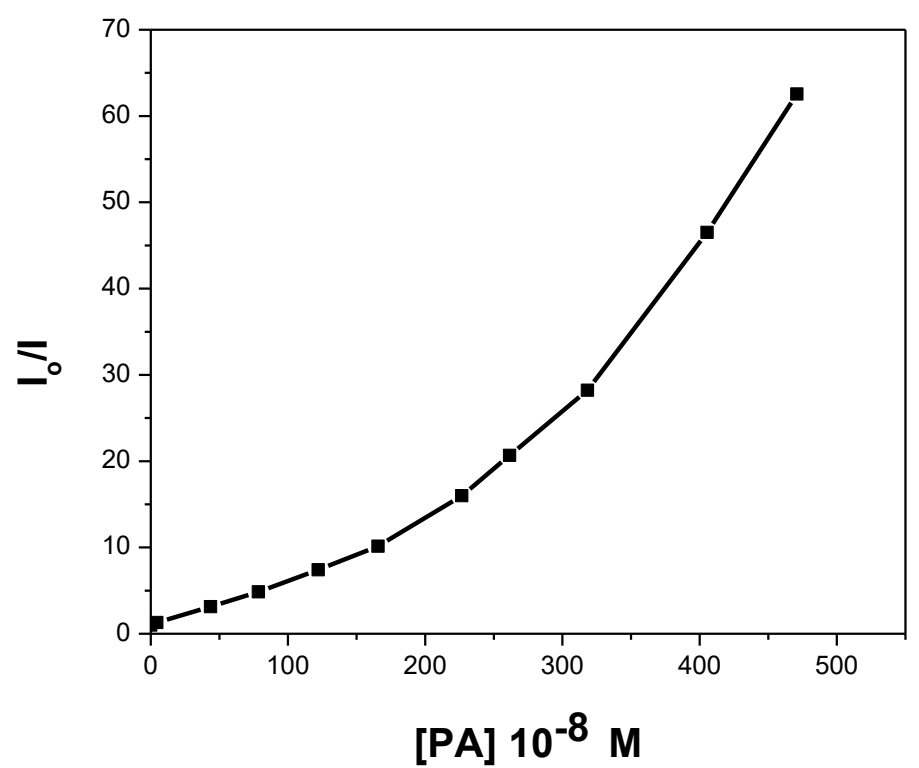

Fig S3. Stern-Volmer plots of polymer P1 corresponding to PA. $\mathrm{I}_{\mathrm{o}}$ is the emission intensity in $\mathrm{H}_{2} \mathrm{O} / \mathrm{THF}$ mixture containing $90 \%$ of water.

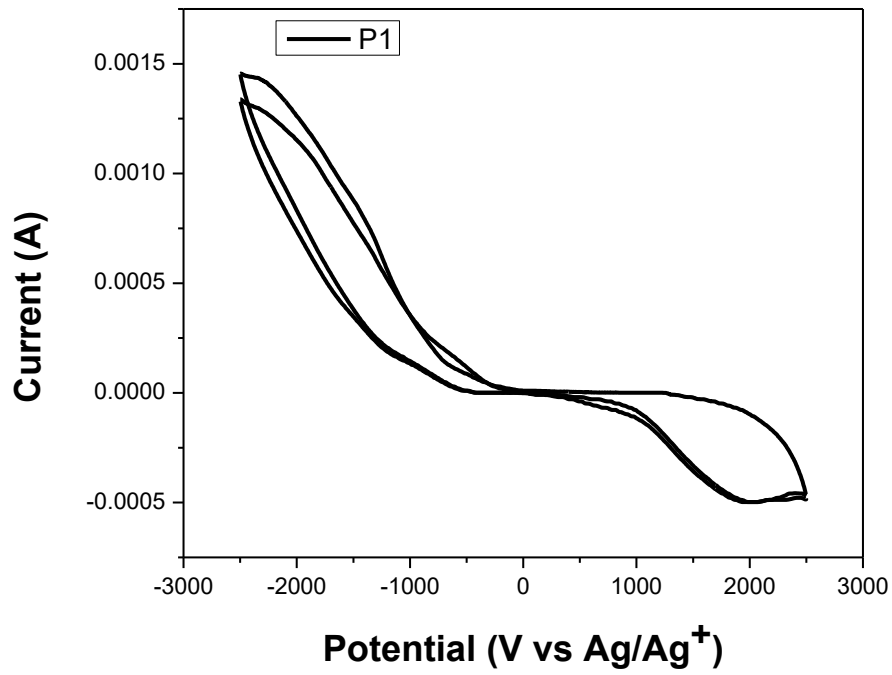

Fig S4. Cyclic voltammograms of the polymer films $(\mathbf{P 1})$ on Pt electrode in $0.1 \mathrm{~mol} / \mathrm{L} \mathrm{Bu}_{4} \mathrm{CF}_{6}$, $\mathrm{CH}_{3} \mathrm{CN}$ solution with a scan rate of $100 \mathrm{mV} / \mathrm{s}$. 


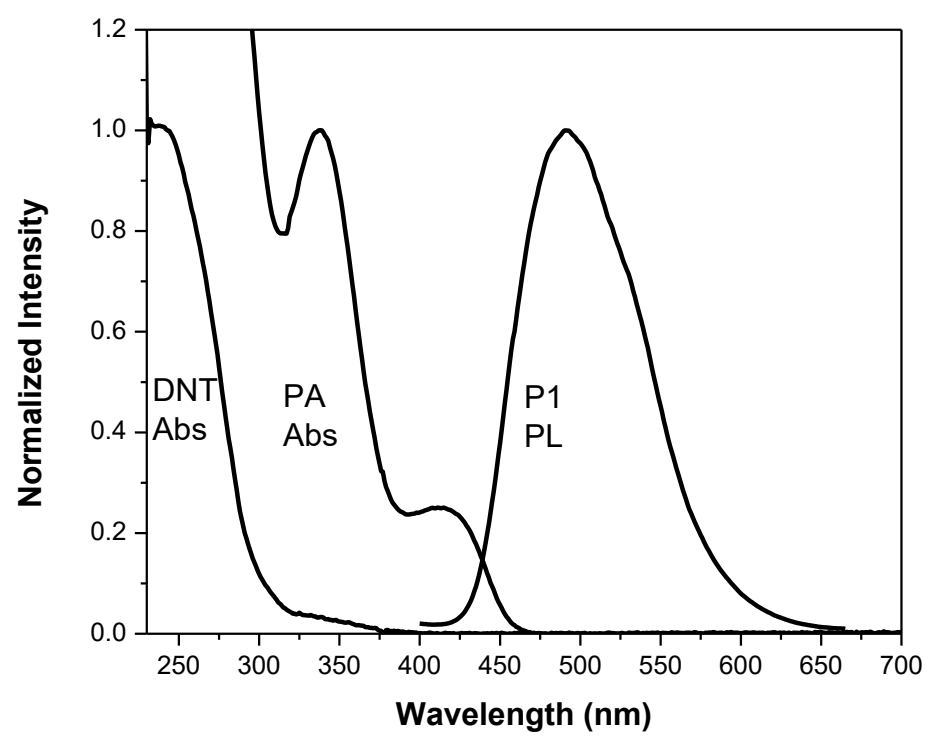

Fig S5.Normalized absorption of DNT, PA and normalized PL of polymer P1

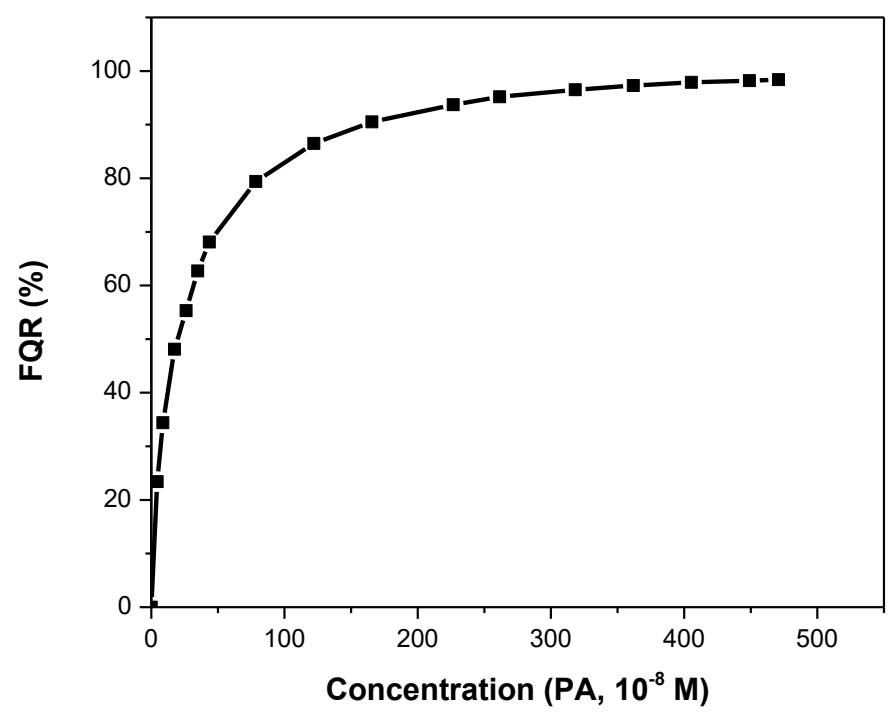

Fig S6. Fluorescence quenching response of polymer $\mathbf{P 1}$ in $90 \% \mathrm{H}_{2} \mathrm{O}-\mathrm{THF}$ mixture with respect to $\mathrm{PA}$ at different concentration 


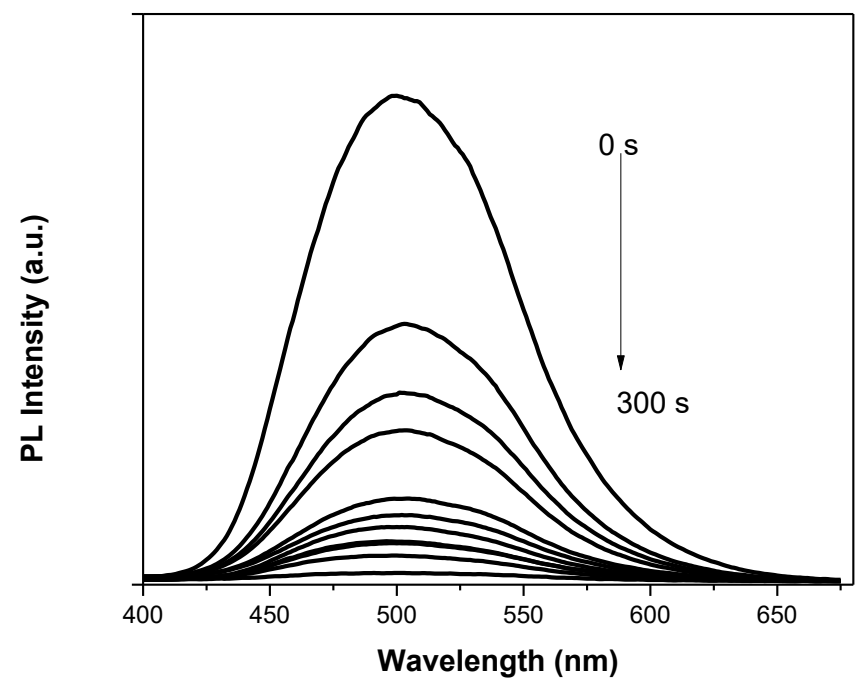

Fig S7. Time-dependent PL spectra of spin-coating film of P1 upon exposure to DNT vapor at room temperature for 0 to 360 s. Film thickness: 4 nm; excitation wavelength: $375 \mathrm{~nm}$

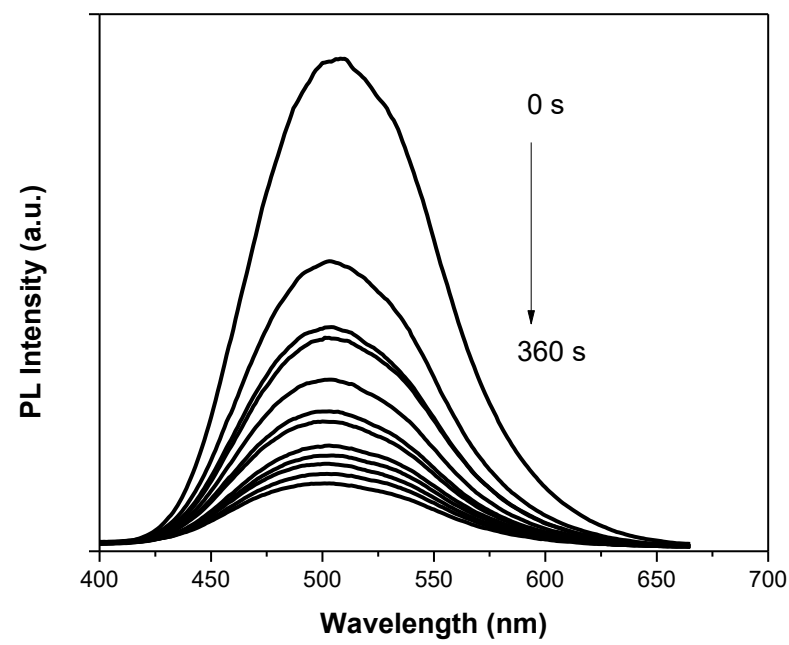

Fig S8. Time-dependent PL spectra of the spin-coating film of P1 upon exposure to DNT vapor at room temperature for 0 to 360 seconds. Film thickness: $22 \mathrm{~nm}$; Excitation wavelength: $375 \mathrm{~nm}$ 


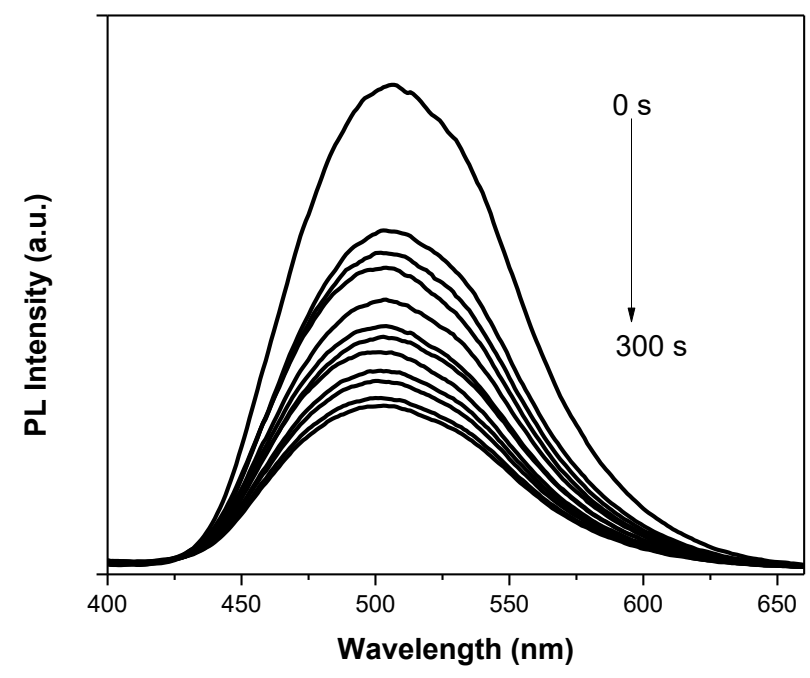

Fig S9. Time-dependent PL spectra of the spin-coating film of P1 upon exposure to DNT vapor at room temperature for 0 to 360 seconds. Film thickness: $75 \mathrm{~nm}$; Excitation wavelength: $375 \mathrm{~nm}$ 